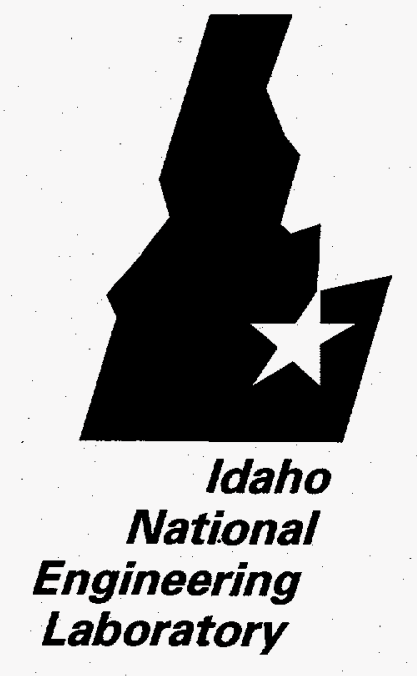

$$
\begin{gathered}
\text { FEDE: ED } \\
\text { Nor } 2 \text { I } 1995 \\
\text { OSTI }
\end{gathered}
$$

\title{
Version 1.00 Programmer's Tools Used in Constructing the INEL RML/Analytical Radiochemistry Sample Tracking Database and its User Interface
}

D. A. Femec 


\section{Version 1.00 Programmer's Tools Used in Constructing the INEL RML/Analytical Radiochemistry Sample Tracking Database and its User Interface}

D. A. Femec

Published September 1995

Idaho National Engineering Laboratory Nuclear Engineering Department Lockheed Idaho Technologies Company Idaho Falls, Idaho 83415-7111 


\begin{abstract}
This report describes two code-generating tools used to speed design and implementation of relational databases and user interfaces: CREATE_SCHEMA and BUILD_SCREEN. CREATE_SCHEMA produces the SQL commands that actually create and define the database. BUILD_SCREEN takes templates for data entry screens and generates the screen management system routine calls to display the desired screen. Both tools also generate the related FORTRAN declaration statements and precompiled SQL calls. Included with this report is the source code for a number of FORTRAN routines and functions used by the user interface. This code is broadly applicable to a number of different databases.
\end{abstract}

\title{
DISCLAIMER
}

\footnotetext{
This report was prepared as an account of work sponsored by an agency of the United States Government. Neither the United States Government nor any agency thereof, nor any of their employees, makes any warranty, express or implied, or assumes any legal liability or responsibility for the accuracy, completeness, or usefulness of any information, apparatus, product, or process disclosed, or represents that its use would not infringe privately owned rights. Reference herein to any specific commercial product, process, or service by trade name, trademark, manufacturer, or otherwise does not necessarily constitute or imply its endorsement, recommendation, or favoring by the United States Government or any agency thereof. The views and opinions of authors expressed herein do not necessarily state or reflect those of the United States Government or any agency thereof.
} 


\section{DISCLAIMER}

Portions of this document may be illegible electronic image products. Images are produced from the best available original document. 


\section{CONTENTS}

1. INTRODUCTION $\ldots \ldots \ldots \ldots \ldots \ldots \ldots \ldots \ldots \ldots \ldots \ldots \ldots \ldots \ldots \ldots \ldots \ldots$

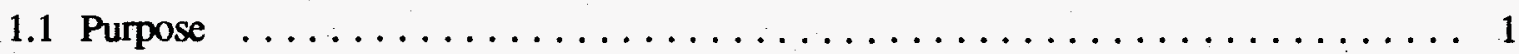

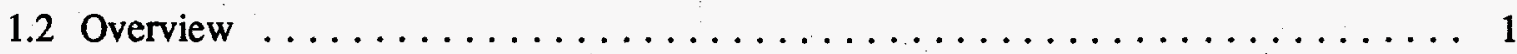

1.3 Definitions, Acronyms, and Abbreviations $\ldots \ldots \ldots \ldots \ldots \ldots \ldots \ldots \ldots$

1.4 Registered Trademarks and Copyrights $\ldots \ldots \ldots \ldots \ldots \ldots \ldots \ldots \ldots \ldots$

2. DESIGN OF THE DATABASE $\ldots \ldots \ldots \ldots \ldots \ldots \ldots \ldots \ldots \ldots \ldots \ldots \ldots \ldots$

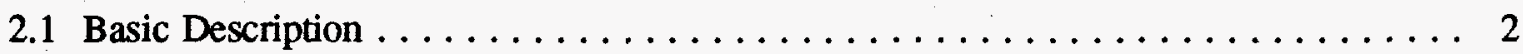

2.1.1 Tables and their Fields and Indexes for Sorting $\ldots \ldots \ldots \ldots \ldots$

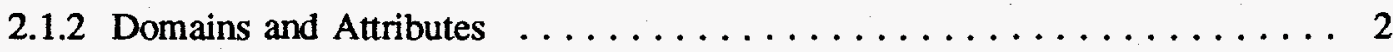

2.1.3 Storage Areas and Snapshot Files $\ldots \ldots \ldots \ldots \ldots \ldots \ldots \ldots \ldots \ldots$

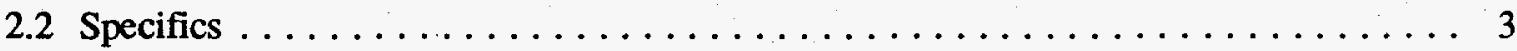

2.2.1 Names of Tables, Storage Areas, and Snapshot Files $\ldots \ldots \ldots \ldots \ldots \ldots$

2.2.2 Names and Descriptions of Domains ............... 3

2.2.3 Backing Up the Database $\ldots \ldots \ldots \ldots \ldots \ldots \ldots \ldots \ldots \ldots \ldots$

2.2.4 Removal of Inactive Entries from the Database $\ldots \ldots \ldots \ldots \ldots \ldots$

3. DESIGN OF THE USER INTERFACE $\ldots \ldots \ldots \ldots \ldots \ldots \ldots \ldots \ldots \ldots \ldots$

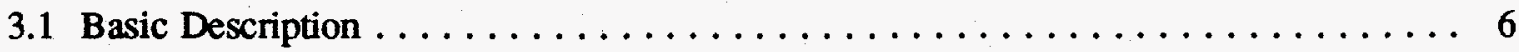

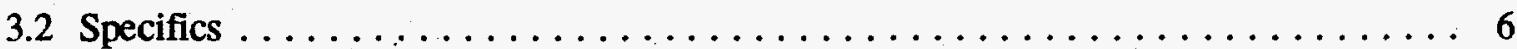

3.2.1 Data Storage Variables and Arrays $\ldots \ldots \ldots \ldots \ldots \ldots \ldots \ldots$

3.2.1.1 Storage of Data $\ldots \ldots \ldots \ldots \ldots \ldots \ldots \ldots \ldots$

3.2.1.2 Conversion of Character Data to Numerical Data . . . . . . . 7

3.2.1.3 Error Checking $\ldots \ldots \ldots \ldots \ldots \ldots \ldots \ldots \ldots$ 
3.2.1.3.1 "a" for Anything $\ldots \ldots \ldots \ldots \ldots \ldots \ldots \ldots \ldots$

3.2.1.3.2 "d" for Date $\ldots \ldots \ldots \ldots \ldots \ldots \ldots \ldots \ldots$

3.2.1.3.3 "l" for Length $\ldots \ldots \ldots \ldots \ldots \ldots \ldots$

3.2.1.3.4 " $t$ " for Time $\ldots \ldots \ldots \ldots \ldots \ldots \ldots$

3.2.1.3.5 "u" for Units of Time $\ldots \ldots \ldots \ldots \ldots \ldots$

3.2.1.3.6 "?" for Questions . . . . . . . . . . . . 9

3.2.2 Communication between the User Interface and the Database ........ 9

3.2.2.1 Forms of SQL Used $\ldots \ldots \ldots \ldots \ldots \ldots \ldots$

3.2.2.2 Commencing a Database Transaction ........ .... 9

3.2.2.3 Add a Sample Entry $\ldots \ldots \ldots \ldots \ldots \ldots \ldots \ldots \ldots$

3.2.2.4 Search for a Sample Entry . . . . . . . . . . . . 11

3.2.2.5 Due Date Checks ... . . . . . . . . . . . 12

3.2.2.6 Update a Sample Entry . . . . . . . . . . . . . 13

4. TOOLS USED IN DESIGNING THE DATABASE $\ldots \ldots \ldots \ldots \ldots \ldots \ldots \ldots$

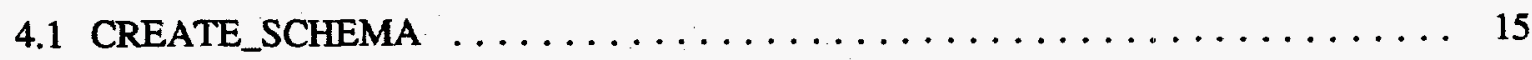

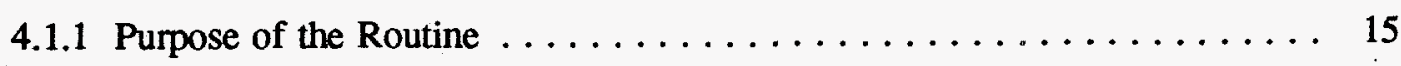

4.1.2 Flow Description of the Routine $\ldots \ldots \ldots \ldots \ldots \ldots \ldots$

4.1.2.1 Creating the Domains $\ldots \ldots \ldots \ldots \ldots \ldots \ldots$

4.1.2.2 Creating the Tables $\ldots \ldots \ldots \ldots \ldots \ldots \ldots \ldots \ldots \ldots \ldots \ldots \ldots$

4.1.2.3 Creating the Schema $\ldots \ldots \ldots \ldots \ldots \ldots \ldots \ldots \ldots \ldots$

4.1.2.4 Associating Attributes with Data Fields $\ldots \ldots \ldots \ldots$

4.1 .3 Input Files $\ldots \ldots \ldots \ldots \ldots \ldots \ldots \ldots \ldots \ldots \ldots \ldots \ldots \ldots$

4.1.3.1 ENTITY.PRN $\ldots \ldots \ldots \ldots \ldots \ldots \ldots \ldots \ldots \ldots \ldots$

4.1.3.2 SCREEN.PRN ................... 17 


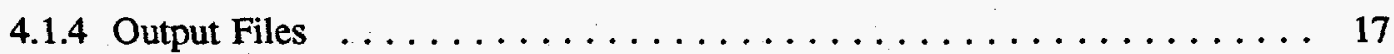

5. TOOLS USED IN DESIGNING THE USER INTERFACE $\ldots \ldots \ldots \ldots \ldots \ldots \ldots \ldots$

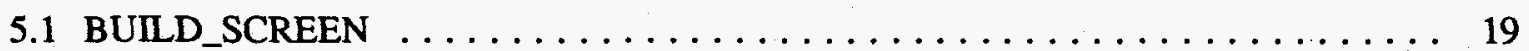

5.1.1 Purpose of the Routine $\ldots \ldots \ldots \ldots \ldots \ldots \ldots \ldots$

5.1.2 Flow Description of the Routine $\ldots \ldots \ldots \ldots \ldots \ldots$

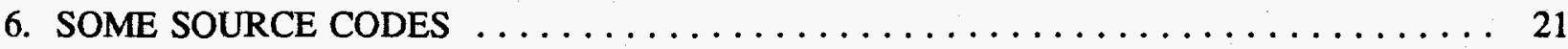

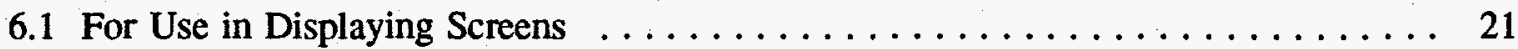

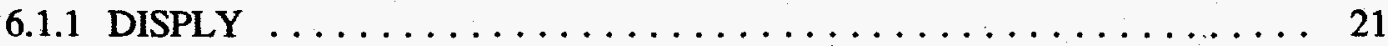

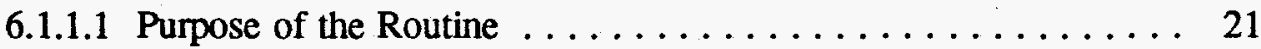

6.1.1.2 Flow Description of the Routine $\ldots \ldots \ldots \ldots \ldots \ldots \ldots$

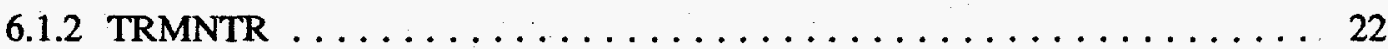

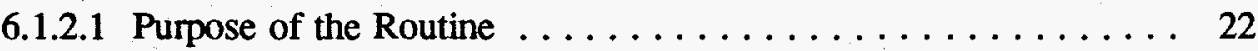

6.1.2.2 Flow Description of the Routine $\ldots \ldots \ldots \ldots \ldots \ldots \ldots \ldots$

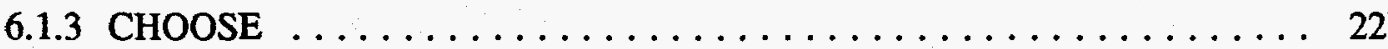

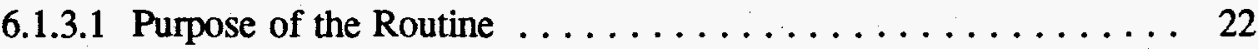

6.1.3.2 Flow Description of the Routine $\ldots \ldots \ldots \ldots \ldots \ldots$

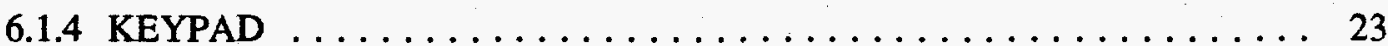

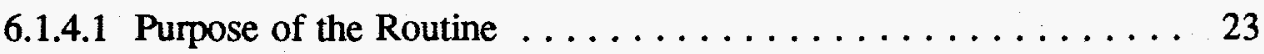

6.1.4.2 Flow Description of the Routine $\ldots \ldots \ldots \ldots \ldots \ldots \ldots$

6.2 For Use in Data Type Conversions $\ldots \ldots \ldots \ldots \ldots \ldots \ldots \ldots \ldots \ldots \ldots \ldots$

6.2.1 Character String-to-Numerical Value Conversions: RVALUE . . . . . . 23

6.2.1.1 Purpose of the Function $\ldots \ldots \ldots \ldots \ldots \ldots \ldots \ldots$

6.2.1.2 Flow Description of the Function $\ldots \ldots \ldots \ldots 23$ 
6.2.2 Numerical-to-Chi..eter String Representation Conversions: CVALUE . . 24

6.2.2.1 Purpose of the Function $\ldots \ldots \ldots \ldots \ldots \ldots \ldots$

6.2.2.2 Flow Description of the Function $\ldots \ldots \ldots \ldots \ldots \ldots$

6.2.3 Conversions between mmddyy Dates and Integer Date Counts $\ldots \ldots \ldots 25$

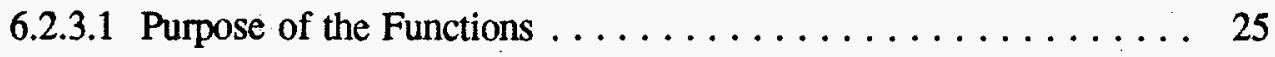

6.2.3.2 Flow Description of the Routine $\ldots \ldots \ldots \ldots \ldots$

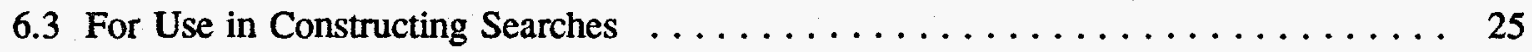

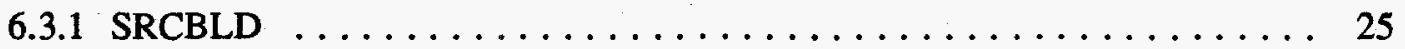

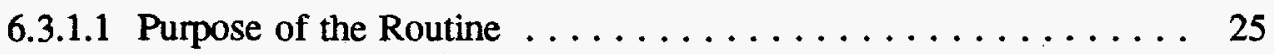

6.3.1.2 Flow Description of the Routine $\ldots \ldots \ldots \ldots \ldots$

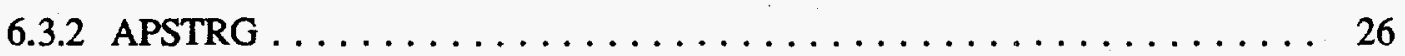

6.3.2.1 Purpose of the Routine $\ldots \ldots \ldots \ldots \ldots \ldots \ldots$

6.3.2.2 Flow Description of the Routine $\ldots \ldots \ldots \ldots \ldots$

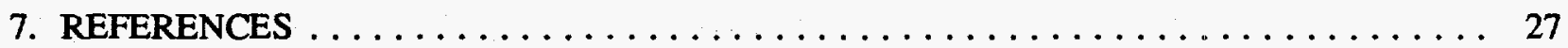

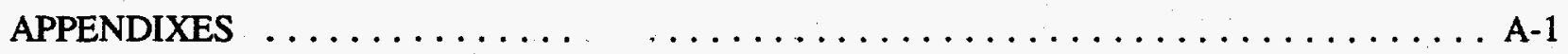

A. CREATE_SCHEMA $\ldots \ldots \ldots \ldots \ldots \ldots \ldots \ldots \ldots \ldots \ldots \ldots \ldots \ldots$ A-1

B. LOTUS® $1-2-3 \otimes$ PRINT FILES $\ldots \ldots \ldots \ldots \ldots \ldots \ldots \ldots \ldots \ldots \ldots$

B.1. Entity-Sorted List $\ldots \ldots \ldots \ldots \ldots \ldots \ldots \ldots \ldots \ldots$ B-1

B.2. Screen-Sorted List $\ldots \ldots \ldots \ldots \ldots \ldots \ldots \ldots \ldots \ldots \ldots$ B-6

C. EXECUTION OF CREATE_SCHEMA $\ldots \ldots \ldots \ldots \ldots \ldots \ldots \ldots \ldots \ldots$ C-1

D. SQL INSTRUCTION SET $\ldots \ldots \ldots \ldots \ldots \ldots \ldots \ldots \ldots \ldots \ldots \ldots \ldots \ldots \ldots \ldots$

E. BULLDSCREEN $\ldots \ldots \ldots \ldots \ldots \ldots \ldots \ldots \ldots \ldots \ldots \ldots \ldots \ldots \ldots$

F. SCREEN TEMPLATE FOR THE GROSS ALPHA-BETA ANALYSES $\ldots \ldots \ldots \ldots$ F 1 
G. BUILD_SCREEN OUTPUT $\ldots \ldots \ldots \ldots \ldots \ldots \ldots \ldots \ldots \ldots \ldots$ G-1

H. ARRAY AND SCALAR DECLARATIONS $\ldots \ldots \ldots \ldots \ldots \ldots \ldots \ldots$

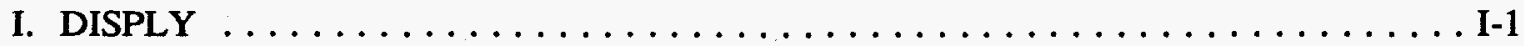

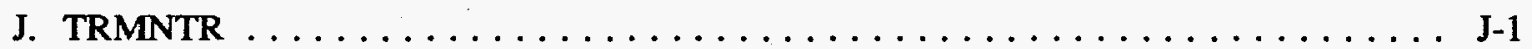

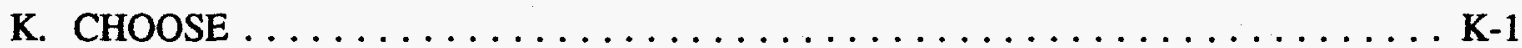

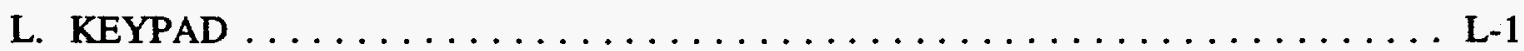

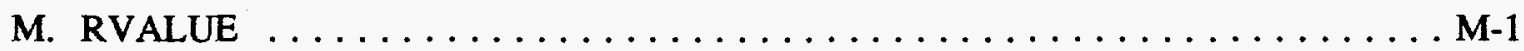

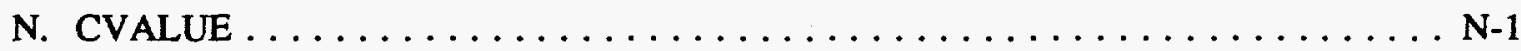

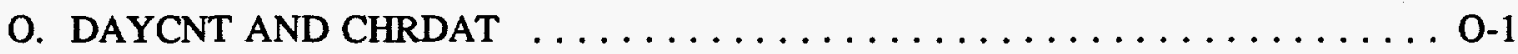

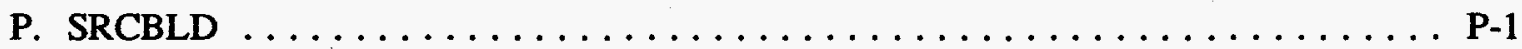

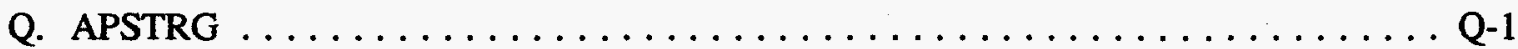

TABLES

Table 1. Arrays and Scalars Used in Coding the User Interface. 


\section{INTRODUCTION}

\subsection{Purpose}

This report presents the software tools developed for the construction of the RML/Analytical Radiochemistry sample tracking database and portions of the code for its user interface. Chapters 2 and 3 are taken directly from Reference 1 and are included for completeness and to aid in understanding the tools; keep in mind that notations in these chapters to Reference 2 are to latter portions of this document.

\subsection{Overview}

Two types of tools have been developed: those for design and constructing the database proper and those for preparing code for the user interface. The starting points for the CREATE_SCHEMA tool are data files prepared using a spreadsheet program; these files name and describe the various data fields necessary for each database table. This tool then constructs an instruction listing for creating the database, as well as FORTRAN declaration statements specifying the entities and attributes that correspond to the various data entry fields and some FORTRAN code for accessing the database. For the tool BUILD_SCREEN, the input is a programmer-prepared ASCII text file displaying how the data screen should look. From this file, BUILD_SCREEN prepares declaration statements for use in preparing the FORTRAN code for the VAX VMS screen management system routine calls used by the user interface.

\subsection{Definitions, Acronyms, and Abbreviations}

Terminology related to Digital Equipment Corporation's VMS operating system, DECnet communication protocol, DECwindows Motif graphical user interface, FORTRAN compiler, GKS (Graphical Kemel System) plotting routines, Rdb relational database software, and SQL Fortran precompiler, is desirable. Otherwise, this document explicitly defines technical terms as they are presented and avoids unnecessary technical jargon for the sake of the general reader.

\subsection{Registered Trademarks and Copyrights}

DEC, DECnet, DECwindows, GKS (Graphical Kernel System), Rdb, VAX, and VMS are registered trademarks of Digital Equipment Corporation (DEC). Motif is a registered trademark of Open Systems Foundation. Lotus and 123 are registered trademarks of Lotus Corporation. 


\section{DESIGN OF E DATABASE}

\subsection{Basic Lescription}

This database is a relational, multifile database. It is created and accessed using the Digital Equipment Corporation's Rdb/MS implementation of the industry standard Structured Query Language (SQL). There is a separate file for each type of analyses and for basic sample information, tracking information, and special instructions. The directory [RDB_DATABASES.SAMPLE_TRACKING] on the user disk of the RML's VAX 6000 mainframe computer contains the database files. The weekly user disk backup includes the contents of these files (see Specifics, section 2.2).

The following subsections present a brief overview of the design of the database. The corresponding subsections under Specifics (section 2.2. present more detail. The presentation assumes the reader has some familiarity with relational database minology.

\subsubsection{Tables and their $F$ is and Indexes for Sorting}

A table (or entity) contains a number of named fields for data storage. The names of the tables are as descriptive as possible while keeping the length of the name within the SQL-standard thirtycharacter limit.

Each field maps to only one data type (or domain). Appropriate fields serve as primary keys (for uniquely identifying each entry in a table) and foreign keys (for connecting entries between tables). Fields can have their own programmer-specified constraints (in addition to those of the corresponding domain) and comments. For example, null entries can be forbidden for a given field, such as the field that serves as the primary key.

A single field or a collection of fields serves as the primary key index for sorting each table. The name of this index is of the form <storage area name>_PRIMARY_KEY_INDEX. Its size depends on the fields which compose it. The comment for this index usually states the table for which it is the sorting key.

\subsubsection{Domains and Attributes}

A minimal set of data types, or domains, describes the space requirements for data in the database. Associated with each data type are a default value, constraints on the acceptable values, and a comment, all of which are programmer-specified.

Attributes are the data-containing fields within the database tables. Their names are as descriptive as possible while keeping the length of the name within the SQL-standard thirty-character limit. Each attribute's data type maps to only one domain.

\subsubsection{Storage Areas and Snapshot Flles}

A separate storage area file contains each table and its definitions and constraints. The name of a storage area file is of the form <storage area name>.RDA. Its initial size is determined by the amount of data to be contained in a single entry to the table times an estimate of the number of entries that need to be available in the working database. The storage areas (and snapshot) files are automatically increased 
in size as needed by Rdb/VMS. (<storage area name> is a concatenation of the first two letters of each underscore-separated part of the table's name (and so can be computer-generated). For example, <storage area name $>$ for the table SAMPLE_INFORMATION is SAIN. The name of the table's snapshot file is of the form <storage area name $>$.SNP.)

\subsection{Specifics}

A listing of the SQL instruction set for creating this multifile database appears in Appendix A. The CREATE_SCHEMA tool generated this instruction set. (The software tools used to design the database are described in Reference 2. The examples presented in that report make use of this database.) The Lotus@ 1-2-3@ print files, ${ }^{a}$ containing the entity- (table-) and screen-sorted attribute description lists, that serve as input to CREATE_SCHEMA appear in Appendix B. A recording of the terminal session from the execution of CREATE_SCHEMA, specifying the programmer's input for this database, appears in Appendix C.

The schema for this database is SAMPLE_TRACKING and so the name of the root file is SAMPLE_TRACKING.RDB. The root file contains the complete set of domain and table definitions.

\subsubsection{Names of Tables, Storage Areas, and Snapshot Flles}

The following are the table names, with the respective storage area names in parentheses:

- SAMPLE_INFORMATION (SAIN)

- for alpha analyses: ALPHA_URANIUM (ALUR), ALPHA_THORIUM (ALTH), ALPHA_PLUTONIUM (ALPL), ALPHA_AM241_SANS_PU239 (ALAMSAPU), ALPHA_AM241_WITH_PU239 (ALAMWIPU), ALPHA_TOTAL_SPECTROMETRIC (ALTOSP), and ALPHA_OTHER (ALOT)

- for gross alpha-beta analyses: GROSS_ALPHA_BETA_AIR_FILTERS (GRALBEAIFI) and GROSS_ALPHA_BETA_AIR_OTHER (GRALBEOT)

- for beta analyses: BETA_STRONTIUM_90 (BEST90), BETA_STRONTIUM_89_AND_90 (BEST89AN90), BETA_TOTAL_STRONTIUM (BETOST), BETA_TRITIUM (BETR), and BETA_OTHER (BEOT)

- for gamma analyses: GAMMA_SCREEN (GASC), GAMMA_FULL_ISOTOPIC (GAFUIS), and GAMMA_OTHER (GAOT)

- SPECIAL_INSTRUCTIONS (SPIN) and

- for possessors: TRACKING (TR).

\subsubsection{Names and Descriptions of Domains}

The following lists the domains and their respective descriptions. Many of the names fully

a. The use of Lotus@ 1-2-3@ in constructing this database does not constitute an endorsement of Lotus@ 1-230. Other spreadsheet programs could also be used to generate these output files. 
describe the data type of the domain. For example, read "at most $X$ alphanumerics" as the description when "X_CHARACTERS" is the name of the domain.

- CHARACTER_TIME_HH_MM (four or five numbers-plus-colon long and of the form hh:mm; for recording times-of-day)

- CHARGE_NUMBERS (exactly nine alphanumerics long)

- FIFTY_CHARACTERS

- FLAGS (one character long; for Yes/No data)

- FORTY_CHARACTERS

- IDENTIFICATION_CODES (exactly twelve alphanumerics long and of the numerical form mmddyyhhmmss; for the sample tro ding databa dentification code)

- INTEGER_DATE_COUNT (date count start. at January 1, 1990; for use in "Due Date Check")

- INTEGER_NUMBERS

- NAMES (at most twenty-five alphanumerics long)

- PHONE_NUMBERS (at most twelve alphanumerics long)

- REAL_NUMBERS

-RML_SPECTRAL_ID_CODES tractly fourteen alphanumerics long and of the form <save area abbreviation of two characters in th $>\$<$ spectral identification code of en alphanumerics in length $>$, for the location and ame of spectral data; see Reference

- SIXTEEN_CHARACTERS

- SIXTY_CHARACTERS

- TEN_CHARACTERS

- TWENTY_CHARACTERS

- UNITS (at most five alphanumerics long; for abbreviated units of measure such as $\mathbf{H}$ for hour, $M$ for minute, $\mathrm{G}$ for gram, and $\mathrm{ML}$ for milliliter, note the exclusive use of uppercase letters, consistent with the user interface forcing all letters entered to uppercase)

- USER_NAME (at most thirty alphanumerics long; for tracking who last modified an entry) and

-VMS_DATE_TIME (seventeen or eighteen alphanumerics long and of the form "dd-mmm-yy hh:mm:ss"; for noting when a record was last modified).

\subsubsection{Backing Up the Database}


DCL BACKUP does not work correctly on the database files (the root file and storage area and snapshot files), owing to their internal structure. These files are all marked for no backup using the DCL command SET FILE /NOBACKUP. This way the weekly backup of the user disk on the VAX 6000 mainframe computer does not include these files.

The weekly backup includes the contents of the database storage area files, though. The routine used by the RML operators to perform the backup contains code to do this (see Appendix D). The routine

(i) verifies the integrity of the database

(ii) performs an RMU BACKUP on the database (this a creates file than can be correctly saved and restored using DCL BACKUP

(iii) performs the DCL BACKUP

(iv) purges all but the two most recent versions of the file generated by RMU BACKUP and

(v) marks these files for no backup.

\subsubsection{Removal of Inactive Entries from the Database}

An inactive entry is one for which all work has been completed, say, at least six months ago. The inactive entries could be moved to a second, nearly identical version of the database. At a minimum, the inactive database would differ from the active database in that it would contain the date the entry was moved. The goal of removing inactive entries is to keep the active database as small as it needs to be; unneeded entries slow down searches and retrievals. The actual moving of entries can be performed automatically or by hand. It could be included as part of the backup procedure (see Backing Up the Database). Inactive entries could be made accessible to the user interface by allowing the user to specify the active or inactive database as the one to use. This capability does not exist with version 1.00 of the sample tracking database. 


\section{DESIGN OF THE USER INTERFACE}

\subsection{Basic Description}

Except for informational messages, the interface makes relating to the database transparent to the user. After selecting the desired option (Add a Sample Entry, Update a Sample Entry, ... ), the user fills in screen-displayed forms. The interface then constructs and executes the appropriate SQL calls. Screen management system routines are used for constructing the screen-displayed forms, reading data from them, and flagging entry errors and missing-yet-required information.

Owing to the case-sensitive nature of SQL searches, all alphabetic input to the interface program is forced to uppercase. Numerical input to data fields can be in the form of integer, real, or exponential numbers; the interface chooses the most complete and concise representation for displaying numerical data retrieved from the database.

\subsection{Specifics}

The software tools described in Reference 2 were used in preparing portions of code, such as declaration statements. They were also used to generate most of the dynamic SQL calls and related FORTRAN declaration and data-type conversion statements for the user interface. The examples presented in that report make use of this database.

The following subsections provide more detail on how the user interface stores data, converts data types, and performs error checking on entered data. It also describes the types of SQL calls the user interface uses and how, using SQL calls, it communicates with the database in performing insertions (for adding a sample entry), cursor declarations and fetches (for searching for a sample entry and due date checks), and modifications (for updating a sample entry). The routines and functions mentioned in the following subsections are described in more retail in Reference 2.

\subsubsection{Data Storage Varlables and Arrays}

The array and scalar declaration statements (and some source code) related to the screen management routines are contained in the include file FLDDEF.INC (see Appendix E). These statements were generated using the tools BUILD_SCREEN and CREATE_SCHEMA; the screen templates submitted to BUILD_SCREEN are presented in Appendix F (see Reference 2).

3.2.1.1 Storage of Data. For ease of use with screen management routines, all information for a given screen is saved in a character array (composed of eighty-character-long elements) assigned to that screen. Since SQL calls cannot make use of arrays (and variable names over six characters long are discouraged in Rdb/VMS), the arrays are equivalenced to a set of scalar variables that are of the required length for the appropriate domains. A character array's name is of the form fidat<first letter of the screen's name>; for example, for the Main screen the array's name is fidatM.

BUILD_SCREEN creates a set of scalar variables using a generic naming scheme that only identifies the screen. For example, scalar variables for the main screen's data fields are named fldM $<n>$, where $\langle n\rangle$ is the sequential number of the field within the screen. For clarity, the names of the scalar variables were changed to include some relation to the appropriate attribute.

The new names of the scalar variables begin with $f<$ first letter of the screen's name $>$. The 
remaining four letters, at most, of each name are constructed in a fashion similar to how storage area names are constructed (see Storage Areas and Snapshot Files, section 2.1.3) but using only the first letter of each underscore-separated part of the respective attribute. For example, for the main screen's sample tracking identification code (attribute name SAMPLE_TRACKING_ID) the scalar variable is named fMSTI (Main screen, then SAMPLE, then TRACKING, then ID). (Some hand modifications were required to eliminate duplicate names).

3.2.1.2 Conversion of Character Data to Numerical Data. If the database requires a number for a given attribute, the appropriate translation of character-to-integer or character-to-real is performed. (The result is stored in a scalar variable the name of which begins with an " $\mathrm{i}$ " instead of an " $\mathrm{f}$ " for integer data and with an " $\mathrm{r}$ " instead of an " $\mathrm{f}$ " for real data). The reverse translation is performed for numerical data retrieved from the database and to be displayed via screen management routines. These conversions are handled by the FORTRAN functions RVALUE (for character string-to-real number conversions) and CVALUE (for real number-to-character string conversions). The codes for these functions, again, are presented in Reference 2.

The arrays containing labels for the fields have names of the form field<first letter of the screen's name>. The arrays containing the names of the entities and attribute corresponding to the screen fields have names of the form atrbt<first letter of the screen's name>. There are no scalar equivalents for these arrays. Other arrays and scalars used in the screen management calls are similarly constructed; Table 1 lists all the arrays associated with any screen. (Note that from here on and in the table "<first letter of the screen's name>" is abbreviated " $\diamond$.")

Some of these arrays will be discussed in more detail later, in the following subsection on error checking for example. The implementation of these arrays, and the coding for the error checking, is found in the FORTRAN routine DISPLY (see Reference 2).

3.2.1.3 Error CheckIng. The user interface checks for three types of errors. First, is the data entered short enough to fit into the appropriate field? Each data field has a maximum number of alphanumerics that can be entered into it, mxnoc $\diamond$. Second, is the data required? If the field is rendered in bold print, an entry must be made into the data field before the user can leave that screen. Also, an entry can be required in a field because an entry has been made in a related field: if an entry appears in the "Date Completed" field, for example, an entry would be required in the "Results report citation" field. Third, is the data entered of the type specified by the fityp $<>$ array? While data entered into a field is read into a FORTRAN CHARACTER variable, a real number may be needed for the corresponding attribute in the database. The following data types, as implemented in the FORTRAN routine DISPLY, are presently available.

3.2.1.3.1 "a" for Anything - Any combination of alphanumerics, spaces included and up to the specified maximum length, is allowed.

3.2.1.3.2 " $d "$ for Date - A date in the integer form mmddyy is allowed. The entry will be converted into an integer date count (that is, how many days have elapsed between the specified date and December 31,1989 ) for storage in the database. Integer date counts make due date checks easier to perform, for instance. Conversions are accomplished using the FORTRAN functions DAYCNT (character mmddyy-to-integer date count) and CHRDAT (integer date count-to-character mmddyy).

An entry of this type must be composed of exactly six integers (leading and trailing zeros must be included). The first two digits, $\mathrm{mm}$, represent the month and must fall in the range 01 to 12 (inclusive). The second two digits, dd, represent the day of the month. The last two digits, yy, represent the 
Table 1. Arrays and Scalars Used in Coding the Use erface.

\begin{tabular}{|c|c|c|c|}
\hline Array Name & Description & $\begin{array}{l}\text { Scalar } \\
\text { Equivalent? }\end{array}$ & $\begin{array}{l}\text { Letter for } \\
\text { Equivalent }\end{array}$ \\
\hline field $<$ & contents of the description field & $\mathbf{N}$ & - \\
\hline fidat $<>$ & $\begin{array}{l}\text { contains error-free data (or is null) from the } \\
\text { data field }\end{array}$ & $\mathbf{Y}$ & f \\
\hline atrbt $>$ & $\begin{array}{l}\text { table (entity) and attribute names corresponding } \\
\text { to the screen field }\end{array}$ & $\mathbf{N}$ & - \\
\hline varbl< & $\begin{array}{l}\text { contains the names of scalar variables equiva- } \\
\text { lenced to fidat }<>\text { and to the respective if-null } \\
\text { indicator }\end{array}$ & $\mathbf{N}$ & - \\
\hline fityp $>$ & $\begin{array}{l}\text { allowed data type for the data field ("a" for } \\
\text { alphanumer } \\
\text { g.) }\end{array}$ & $\mathbf{N}$ & - \\
\hline fdlen $<>$ & length of the aescription field & $\mathbf{N}$ & - \\
\hline fdrow $<>$ & $\begin{array}{l}\text { row number for display of the description and } \\
\text { data fields }\end{array}$ & $\mathrm{N}$ & - \\
\hline fdcol $>$ & $\begin{array}{l}\text { starting column number for display of the } \\
\text { description field }\end{array}$ & $\mathbf{N}$ & - \\
\hline ficolo & $\begin{array}{l}\text { starting column number for display of the data } \\
\text { field }\end{array}$ & $\mathbf{N}$ & - \\
\hline mxnoc $>$ & $\begin{array}{l}\text { maximum number of alphanumerics allowed in } \\
\text { the data field (length of data field) }\end{array}$ & $\mathbf{Y}$ & $\begin{array}{l}1 \\
\text { (for length) }\end{array}$ \\
\hline fdrnd $\infty$ & $\begin{array}{l}\text { rendition of the description field (normal if } \\
\text { pptional, bold if required, reverse video if } \\
\text { ixed) }\end{array}$ & $\mathbf{N}$ & - \\
\hline firnd $<>$ & rendition of the data field & $\mathbf{N}$ & - \\
\hline ifnul $\diamond$ & $\begin{array}{l}\text { if-null indicator: }-1 \text { if the data field is empty } \\
\text { (null), } 0 \text { otherwise }\end{array}$ & $\mathbf{Y}$ & $\begin{array}{c}n \\
\text { (for null) }\end{array}$ \\
\hline ifrqd $>$ & $\begin{array}{l}\text { entry into the current data field required if data } \\
\text { field number ifrqd }>\text { is not empty }\end{array}$ & $\mathbf{N}$ & - \\
\hline idito $>$ & $\begin{array}{l}\text { "ditto" transfers contents of data field number } \\
\text { idito }>\text { into the current data field }\end{array}$ & $\mathbf{N}$ & - \\
\hline ifmod $>$ & $\begin{array}{l}\text { if-modified indicator: true if the data field's } \\
\text { value has been changed (during Update), false } \\
\text { otherwise }\end{array}$ & $\mathbf{Y}$ & $\stackrel{\mathrm{m}}{\text { (for modified) }}$ \\
\hline
\end{tabular}

year and are interpreted a lows: if yy is between 90 and 99 , the year is $19 y$; if yy is between 00 and 
79, the year is $20 y y$. Entries between 80 and 89 are not allowed as they should be interpreted as 20yy, yet the resulting integer date count would be too large to fit into a FORTRAN INTEGER*4 variable and hence into the respective database attribute.

3.2.1.3.3 "I" for Length - Here the entry must be exactly mxnoc $<$ alphanumerics long. There are no restriction as to the alphanumerics used. One instance in which "l" is used is the charge number to be billed for performing the analyses. All charge numbers are exactly nine alphanumerics long, so a shorter or longer entry is invalid.

3.2.1.3.4 " $t$ " for Time - The format of the entry allowed here is either "h:mm" or "hh:mm." $h$ or hh represents the hour as an integer. A leading zero is not necessary because the colon can be used to delineate the hour portion of the entry from the minute portion. $\mathrm{mm}$ represents the number of minutes.

3.2.1.3.5 " $u$ " for Units of Time - In instances such as the units for the duration of the spectral acquisition, an entry of either $\mathrm{H}$ for hours or $\mathrm{M}$ for minutes is allowed. "u" covers only units of time (duration); it does not cover sample size units, for example.

3.2.1.3.6 "?" for Questions needing a Yes-or-No Answer - The only allowed entries here are " $\mathrm{Y}$ " for yes and "N" for no. This data type is used for flags such as whether a certain type of analysis is to be performed.

\subsubsection{Communication between the User Interface and the Database}

SQL calls are used to transfer data between the user interface and the database. These calls are either hard-coded into the user interface (precompiled SQL) or are constructed by it (dynamic SQL).

3.2.2.1 Forms of SQL Used. Both precompiled SQL and dynamic SQL calls are used. Precompiled SQL calls are completely known by the user interface before they are executed. Such calls include inserting all attributes of an entry into the database. An EXEC SQL preface marks these calls as precompiled SQL statements rather than as statements in the host program's language.

Dynamic SQL calls can only be composed during execution of the user interface. Instances of these include requesting the tables needed for the current transaction. The instruction set for a dynamic SQL call is constructed as a FORTRAN CHARACTER variable and is executed from the main routine using EXEC SQL EXECUTE IMMEDIATE. To minimize the length of the character string, a FORTRAN routine APSTRG (APpend character STRinG) fills the character variable with each additional portion of the SQL call passed to it.

Modular SQL, in which calls are made from the main routine to SQL routines constructed in a single and separate file, is not used. The main routine of the user interface is larger than might seem prudent for ease of code development because of the Rdb/VMS requirement that all precompiled SQL statements reside in the same routine.

3.2.2.2 Commencing a Database Transaction. Communications with the database occur within transactions. The transaction definition (SET TRANSACTION) specifies the tables of the database the user needs and the type of access required. For example, for adding an entry to the database, the sample coordinator needs exclusive write access to all of the tables for the requested analyses. No one else should be able to access these tables in any fashion until the addition is completed. The construction of the SET TRANSACTION call depends on the application (for example, see Add a Sample Entry, 
section 3.2.2.3).

At present, all SET TRANSACTION calls will wait at most sixty seconds for the requested tables to become available. To minimize conflicts (lock conditions resulting from two users wanting the same table at the same time and in incompatible fashions), database transactions last only as long as necessary. Any database transaction starts only as soon as all the requisite information is available. Again using the example of adding an entry, the database transaction does not begin until all of the appropriate screens are filled by the user. And, accordingly, each transaction is closed - either committed, that is, saved, or rolled back, that is, undone - upon completion.

3.2.2.3 Add a Sample Entry. The addition (insertion) of a sample entry to the database occurs via the SQL INSERT command. Here the main routine constructs the SET TRANSACTION command (using the FORTRAN routine APSTRG mentioned above in Forms of SQL Used), and then executes it as a dynamic SQL call (EXECUTE IMMEDIATE). Again, dynamic SQL is used since all of the tables that will be needed are generally not known until it is time for the addition to occur. All tables needed are included in a single SET TRANSACTION command because violations of constraints are better managed within a single transaction than over a series of transactions. A constraint, for example, may require that an entry for sample identification code exists in the sample information table before an entry for that same identification code can be made into an analysis table. A typical sequence of calls to APSTRG, followed by execution of the SET TRANSACTION call, would be

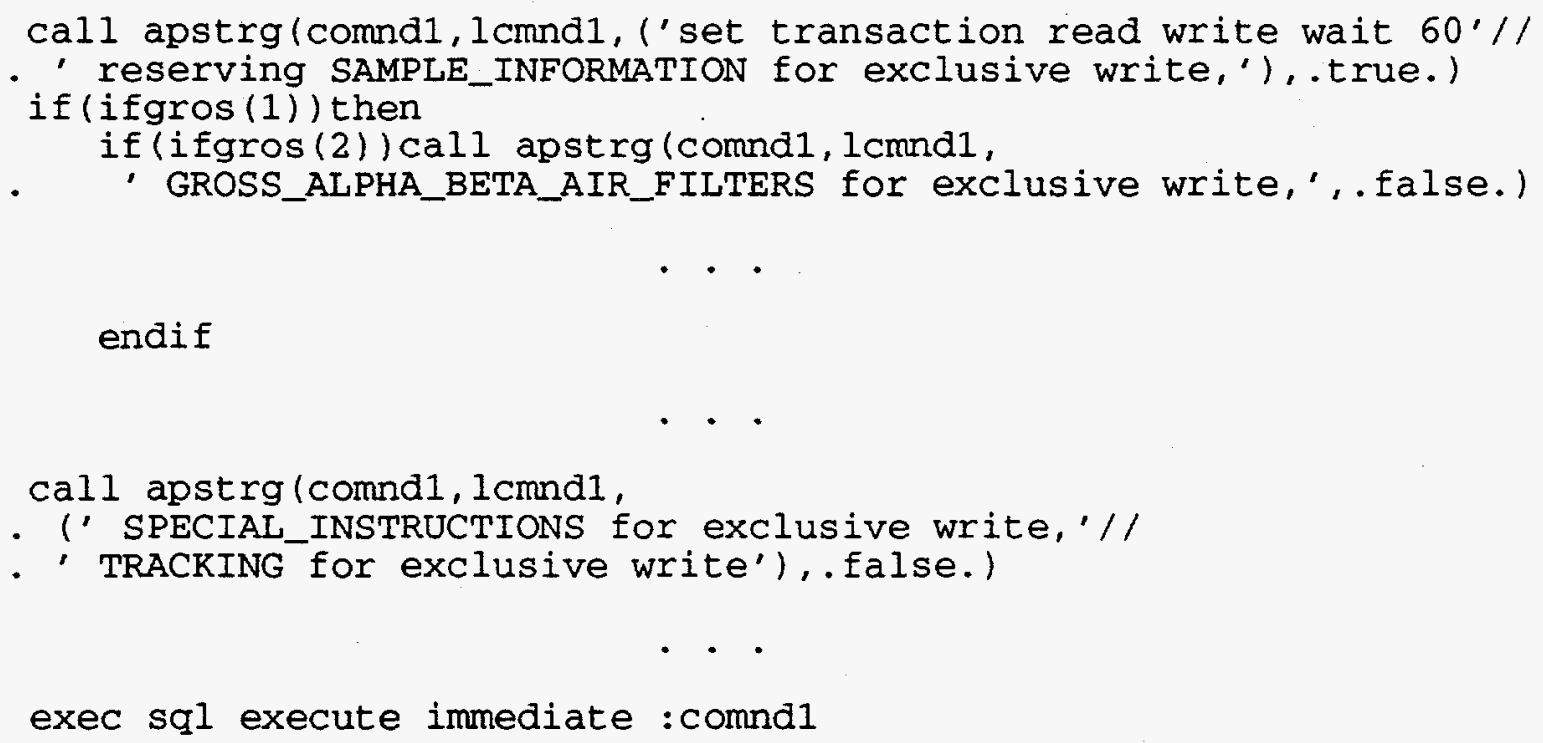

The next SQL calls are precompiled SQL calls and insert the data provided by the sample coordinator into the database:

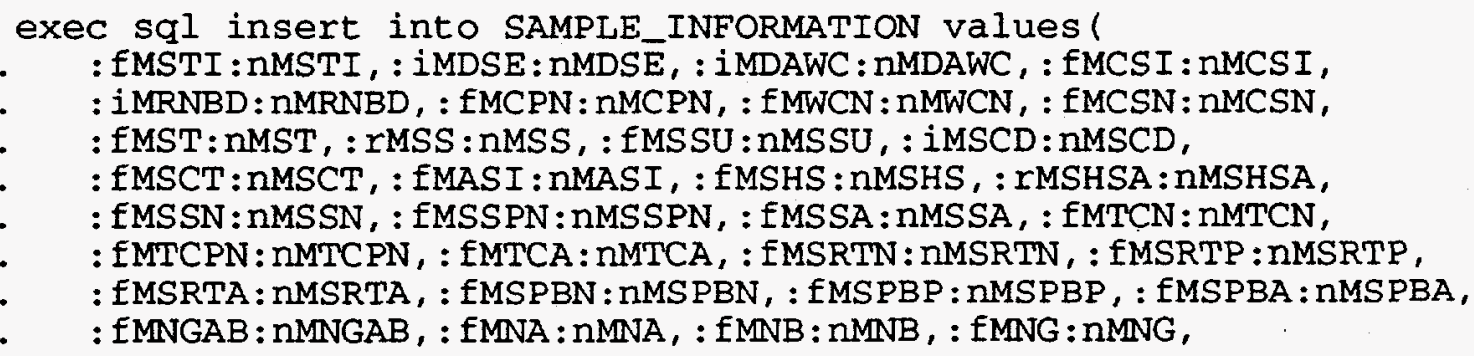




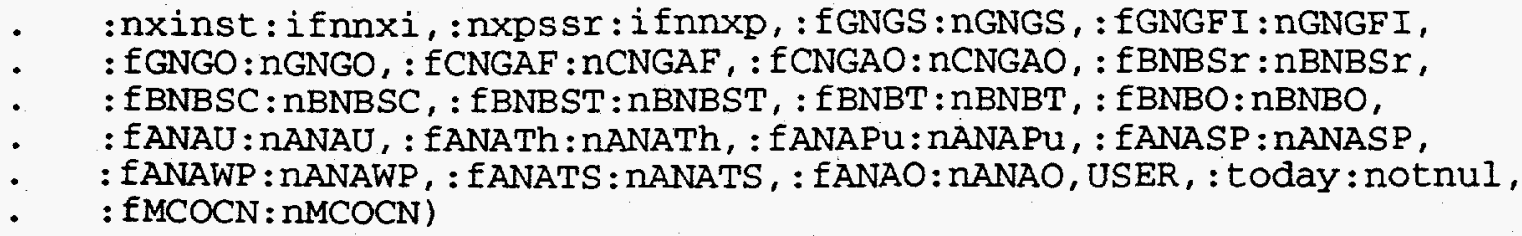

The values are passed in the form :<scalar equivalent of data field $>:<$ scalar equivalent of if-null indicator $>$. The colon preceding each variable name tells the SQL precompiler that what follows is a variable in the user interface's native language, not an SQL variable or key word.

What about fields into which no entry was made? Recall that there is an if-null indicator array. If a field is left blank, that field's if-null indicator is set (to a value of -1). When this if-null indicator is passed to the database (with a value of -1), the attribute in the database entry is marked NULL. (The ifnull indicator could be used to specify which attributes are to be passed to a table. Dynamic SQL would be used in place of precompiled SQL in this case. Pointers to variables are required in place of variables for dynamic SQL, though.)

3.2.2.4 Search for a Sample Entry. This is another instance where dynamic SQL calls could be used; this time they are, in conjunction with the APSTRG routine and the atrbt $>$ and fidat $>$ arrays. To perform a search, the user fills in all the fields necessary to describe the entry or entries desired. The if-null indicator is used to indicate the fields to be included in the search command. The user is to leave blank those fields for which no information is known or for which the information may vary among the desired entries.

Recall that the atrbt $>$ arrays contain the name of the table (entity) and attribute that correspond to a given screen field. The search command is constructed using the atrbt $>$ arrays and the values contained in the fidat $>$ arrays. If a data field contains an entry (and so its if-null indicator is not set), the appropriate elements from atrbt $<>$ and the entry are used in constructing the dynamic SQL search call via APSTRG. These portions of the search command are constructed in the FORTRAN routine SRCBLD (SEARCH BUILD).

Searches are conducted using cursors and fetches from cursors. A cursor can be thought of as a virtual table containing only the specified entries (EXEC SQL DECLARE . . . CURSOR for <statement name>; EXEC SQL PREPARE <statement name> from <search command>). Data from a cursor can be retrieved into native language variables (EXEC SQL FETCH . . . INTO . . .). A typical portion of the main routine's code for constructing the search command and executing the search and retrieval is

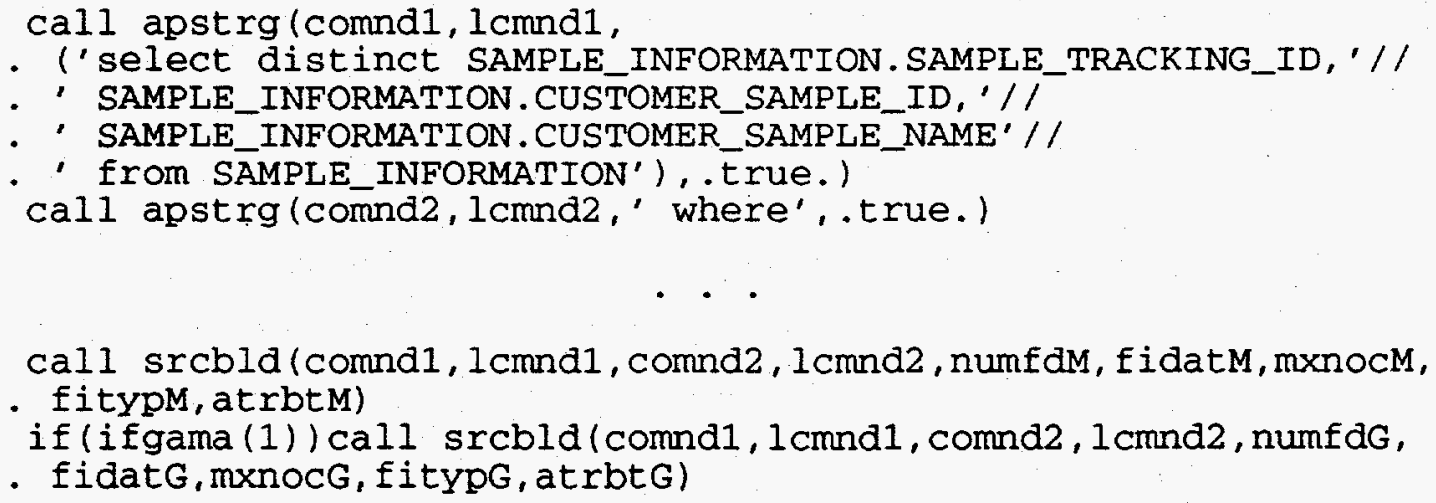




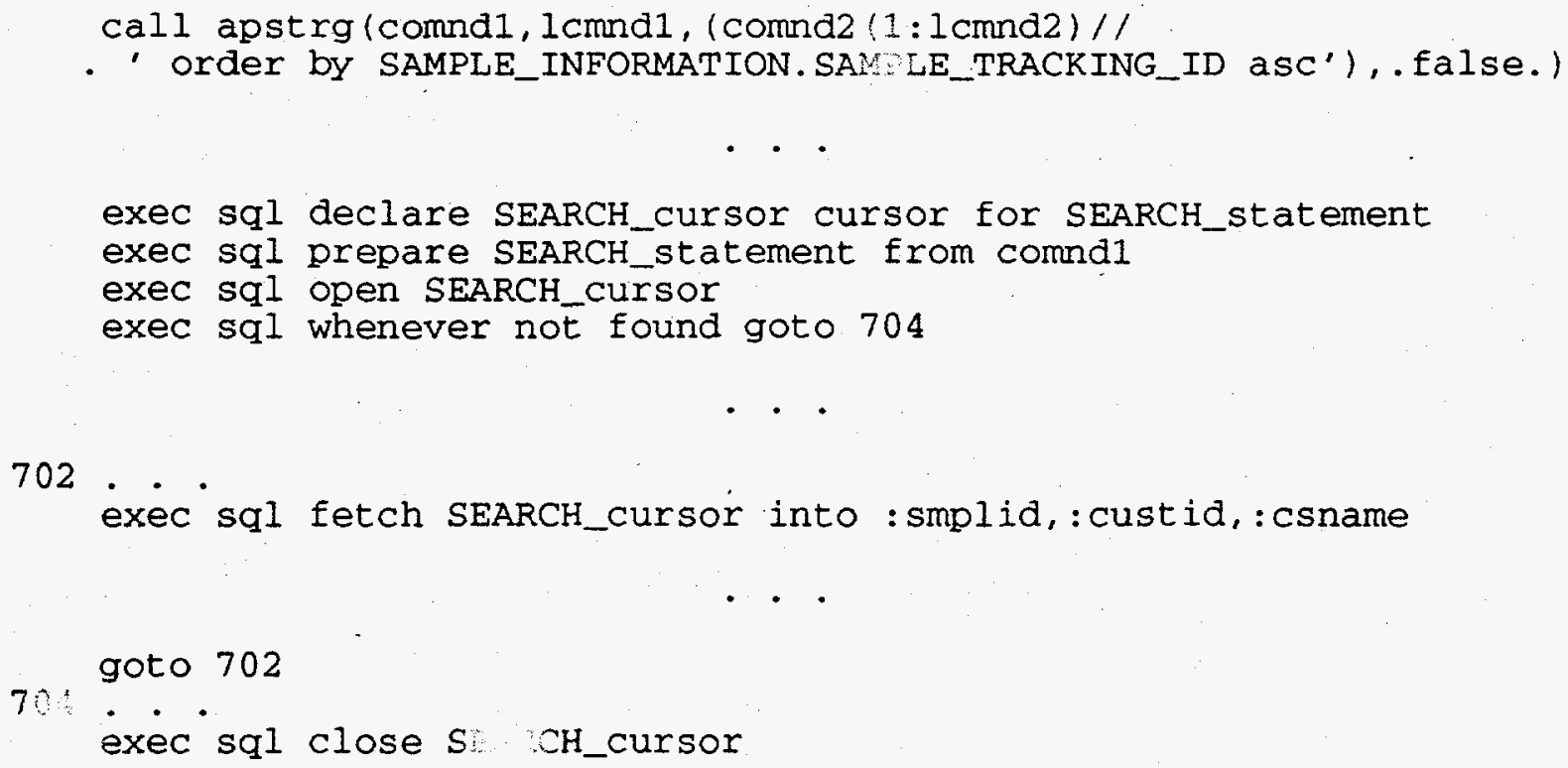

Note that two character gs are input to the routine APSTRG. For each non-null data field, a call to SRCBLD transfers the n 2 of the respective entity alone to the first string (if it does not already appear there) and, to the second string, the entity (table) name, the attribute name, and the value contained in the data field. The second string is appended to the first string prior to the search. Finally, the search is performed as the cursor is declared (defined), opened (much as a file is opened), and read from (via FETCH) until the last entry is found (watched for by WHENEVER NOT FOUND . . .). The data retrieved can be displayed as it is found and stored for future reference. After all the data is retrieved from the cursor, the cursor is closed.

3.2.2.5 Due Date Checks. Due date checks are a form of search where the same, specific attributes in the various tables are always interrogated, namely the date-completed fields. The sample coordinator states how many days ahead should be checked for analyses and reports which will be due. The user interface calculates the integer date count for the current day, and adds to it the number of days ahead to be checked. A search is then preformed for all entries having due dates the integer date count for which is less than or equal to the above sum (IDUCNT) and for which the specified work has not been completed (that is, for which the DATE_ALL_WORK_COMPLETED field is null). The coding for one such search is

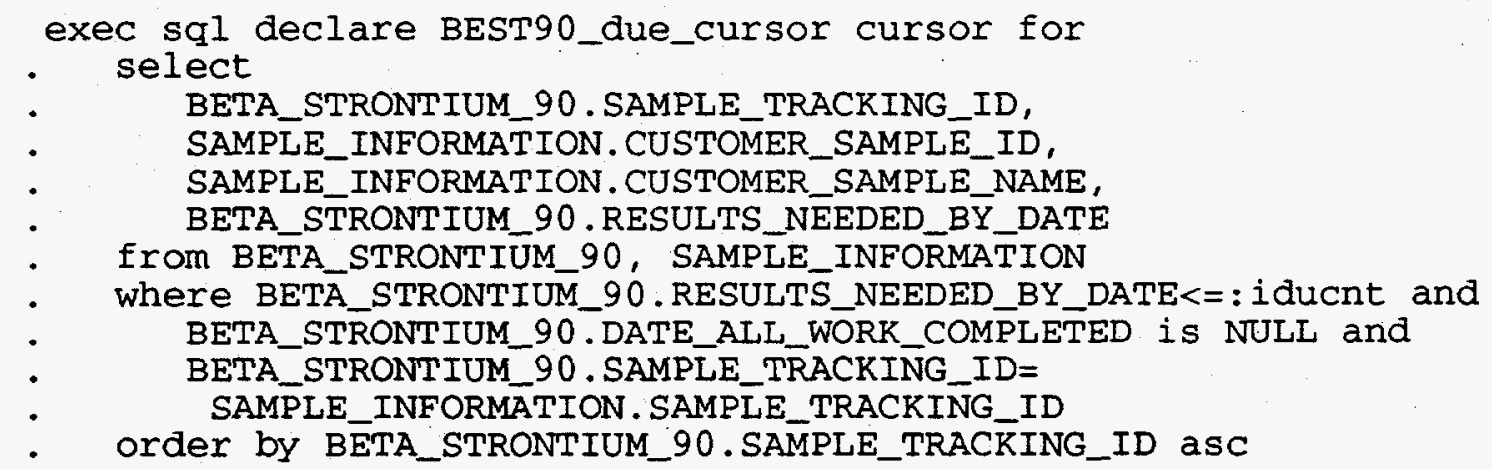


exec sql open BEST90_due_cursor

exec sql whenever not found goto 836

834 exec sql fetch BEST90_due_cursor into :smplid,:custid, :csname,

- :duedat

836 goto 834

exec sql close BEST90_due_cursor

3.2.2.6 Update a Sample Entry. This final mode of communication uses the techniques described above. First, data for the specified entry is fetched from the database via a cursor:

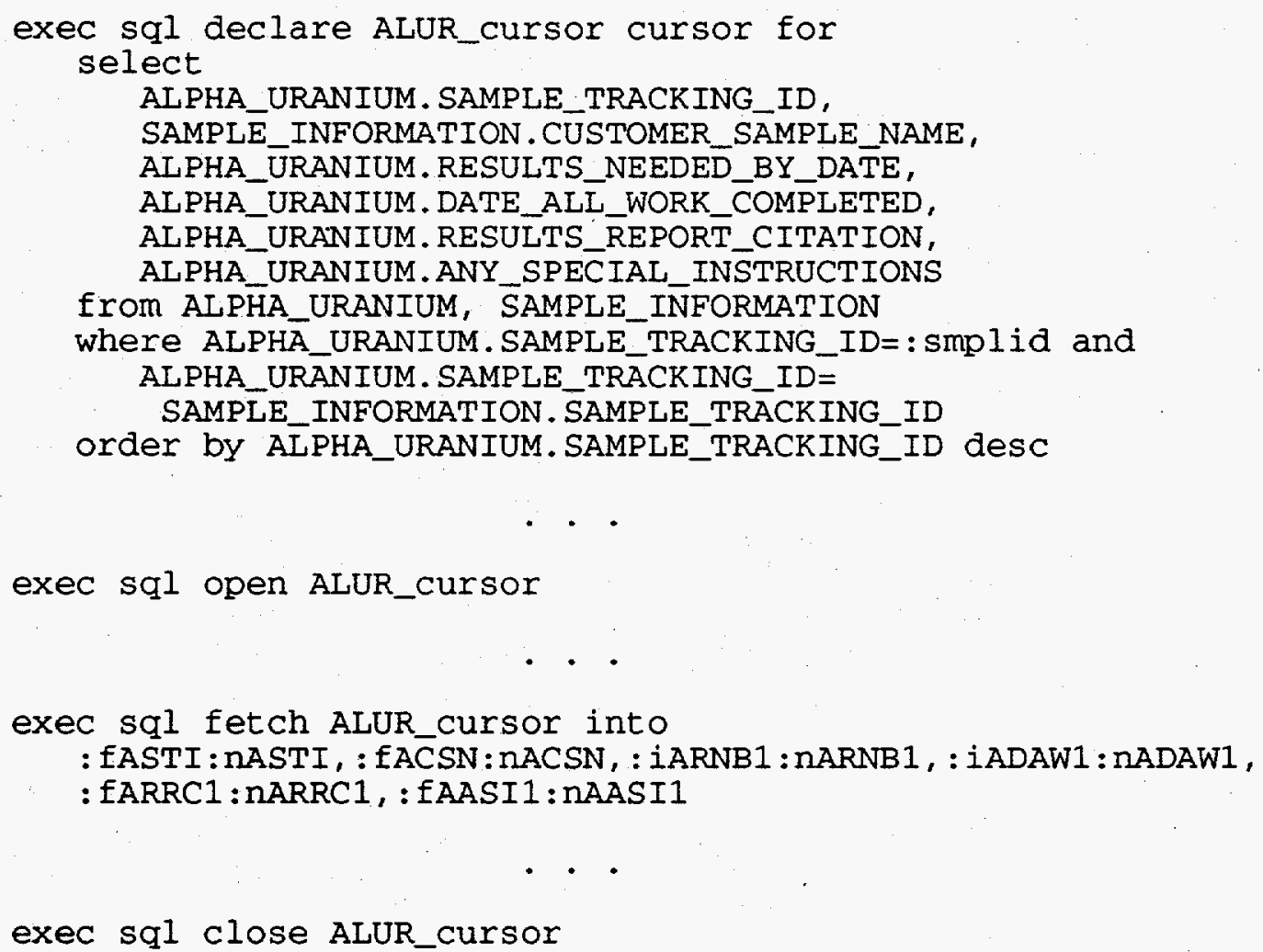

exec sql close ALUR_cursor

The retrieved data is then displayed for the sample coordinator to update. Once the updating of the screens is completed, the entire database entry is updated (modified) if any changes have been made within the screens.

The if-modified indicator plays a role different from that the if-null indicator plays in searching for a sample entry. If any of the if-modified indicators for a table's fields are true, the entire entry is updated if it previously existed in the database. If the entry is new to the database, the entry is added to the database: 
i. falph (2) and. (mARNB1. or .MADAW1. or.MARRC1. or .MAASI1)) then

f(ifalpo(2)) then !Use update to change the entry. exec sql update ALPHA_URANIUM set RESULTS_NEEDED_BY_DATE $=:$ IARNB1 : IARNB1, DATE_ALL_WORK_COMPLETED $=:$ IADAW1: 1 ADAW1, RESULTS_REPORT_CITATION=: FARRC1:nARRC1, ANY_SPECIAL_INSTRUCTIONS=: IAASI1: AAASI1, USER_CREATINTG_OR_MODIFYING=USER, DATE_CREATED_OR_MODIFIED $=:$ today : notnul where SAMPLE_TRACKING_ID=: smplid

else !Use insert since it was not there before. exec sql insert into ALPHA_URANIUM values ( : fASTI: $n A S T I,:$ :ARNB1 : nARNB1, : IADAW1 : NADAW1, endif : fAREC1: nARRC1, AASI1:nAASI1,USER, : today:notnul) endif 


\section{TOOLS USED IN DESIGNING THE DATABASE}

\subsection{CREATE_SCHEMA}

\subsubsection{Purpose of the Routine}

CREATE_SCHEMA generates the SQL commands for creating the database and its associated definitions (the schema). It prepares FORTRAN declaration statements for variables that allow a data entry field to be related to the correct entity and attribute. It also generates the precompiled SQL code (for FORTRAN) necessary to access the database in a number of ways, such as UPDATE and FETCH, INSERT and CURSOR.

CREATE_SCHEMA asks questions of the programmer as it runs, and so it should be executed interactively. To do so, type

\section{@CREATE_SCHEMA}

at the DCL prompt. There are no command line parameters to be specified. Appendix C records the interactive execution of CREATE_SCHEMA used to create the sample tracking database's schema.

\subsubsection{Flow Description of the Routine}

The DCL code for CREATE_SCHEMA is presented in Appendix A and is summarized here. There are no declaration statements in DCL; numbers can be stored only as integers, and character strings are distinguished from numbers by enclosing the value in quotation marks. After initializing some counters, CREATE_SCHEMA opens the two Lotus® 1-2-3® print files, ENTITY.PRN and SCREEN.PRN; these files are presented in Appendix B and described in detail below under Input Files, section 4.1.3.

After obtaining the name of the schema to be created from the programmer, CREATE_SCHEMA opens a number of temporary output files and writes appropriate comments to them. These files will later be concatenated and rearranged to yield the final output files, described below under Output Files, section 4.1.4.

4.1.2.1 Creating the Domains. The first set of SQL commands to be generated create the various domains (or data types) for use within the database. The statements to create two domains, USER_NAME and VMS_DATE_TIME, are automatically written to file by CREATE_SCHEMA. The descriptions of the rest of the domains are provided interactively by the programmer. CREATE_SCHEMA reads through the ENTITY.PRN file until it finds the alphabetic list of unique domains. It then reads one of these at a time, and presents each to the programmer for definition. The programmer can assign the domain to one of four generic data types: character, date, integer, or real. Default values and comments can also be assigned. CREATE_SCHEMA keeps track of the names of the domains and their respective sizes in bytes for later use. From all of this information, CREATE_SCHEMA writes to file the appropriate SQL statements.

4.1.2.2 Creating the Tables. The second set of SQL commands that CREATE_SCHEMA generates create the various entities or tables described in ENTITY.PRN. Starting over at the top of ENTITY.PRN, CREATE_SCHEMA reads the attribute listings for a given entity one at a time. For each entity (or table), an abbreviated name is constructed for later use. The abbreviation is constructed as described above in Storage Areas and Snapshot Files, section 2.1.3. For each new table encountered, 
CREATE_SCHEMA writes the fetches from the database, inser part of a number of precompiled SQL calls (cursor declarations, into the database, . ...).

Each attribute listing spefies the name of the attribute, what type of key, if any, the attribute is, and the domain to which the attribute is to be associated. From the domain, for instance, CREATE_SCHEMA determines how many more bytes of storage space per record will be needed as each attribute is added. (Remember that CREATE_SCHEMA recorded the number of bytes needed for each domain earlier.) If the attribute is labeled as the primary key for the table, CREATE_SCHEMA records this; if multiple attributes make up a table's primary key, CREATE_SCHEMA properly constructs the necessary key description, including the total number of bytes needed for the primary key.

Special handling is needed for those attributes which will contain integer or real numbers in the database. The screen management routines that present the data for the user to see can only make use of characters strings, and so character representations need to be created for numerical data. In these cases, CREATE_SCHEMA generates the appropriate integer or real FORTRAN declaration statements and the code or function calls necessary to convert between numeric and character representations.

Once the end of a table (entity) reached, CREATE_SCHEMA calculates the size of the storage area for the table and its snapshot file. : VISHED_WITH_TABLE is the subroutine that handles these calculations and the generation of the appropriate SQL commands to create the table. The subroutine also completes the precompiled SQL calls that have been growing with each new attribute.

4.1.2.3 Creating the Schema. After all of the statements for creating all of the tables have been generated, CREATE_SCHEMA now has enough information to write the first part of the statements that actually create the schema. The main piece of needed information that was unknown until all of the tables were dealt with is the size of the largest table. Associated with the schema is a buffer, the size of which depends on the size of the largest table.

In should be clear at this point why a number of temporary output files will be put together to arrive at the final output files. Consider just the statement for creating the schema. Rather than forcing a second or even third pass through the print files, CREATE_SCHEMA writes to a file called CREATE_SCHEMA_SECOND.SQL the statements for creating the tables as the tables are found. The first part of the statements cannot be written until later, and so they are written to another file, CREATE_SCHEMA_FIRST.SQL. The final cutput file, CREATE_<name of the schema $>$.SQL, is created by concatenating CREATE_SCHEMA_SECOND.SQL to the end of CREATE_SCHEMA_FIRST.SQL.

A similar approach is taken in dealing with the precompiled SQL statements and the associated representation conversions, and is then taken a step further. In the instance of the precompiled SQL statements that handle the cursor declarations, the contents of one of the files needs to be reordered. Fragments of the precompiled SQL statement are intermingled with the intemal write statements that are used to effect the number-to-character representation conversions, and so the file is read through and the appropriate pieces culled out to new temporary files. These new temporary files are then used in the final concatenation in place of their parent file.

4.1.2.4 Assoclating Attributes with Data Flelds. The last portion of CREATE_SCHEMA makes ise of the SCREEN.PRN Lotus ${ }^{\circledR} 1-2-3 ®$ print file. It generates the FORTRAN declaration state nits that associate the data fields within a screen with the appropriate attributes. It also generates the iaration statements that associate the data fields with the scalar variables that contain the value ent: into that field (see Storage of Data, section 3.2.1.1). 
Each attribute listing includes a screen (identified using the one-letter abbreviations used by BUILD_SCREEN, section 5.1) and the number of the data field within that screen with which the attribute is to be associated. This information, along with the domain, allows CREATE_SCHEMA to generate the proper declaration statements.

\subsubsection{Input Files}

4.1.3.1 ENTITY.PRN. Appendix B.1 contains a listing of the ENTITY.PRN file used to create the schema for the sample tracking database. Attribute lists for different entities are separated from each other by a 30-character-long dashed line. Because duplicate "keys" (see below) have not been removed from the listings, a change in entity name does not necessarily signal the beginning of a new entity.

For the majority of the file there are six columns that contain information about each attribute. The first column, labeled "Entity," contains the name of the entity (or table) in which the attribute can be found. The second column, labeled "Attribute," contains the name of the attribute. (Note that there is actually a one-character-wide column preceding "Attribute." This column contains only a period and was added for readability.)

The third column is labeled "Key" and in many instances is left blank. The only notations made here have to do with whether the attribute is the table's primary key (and so is marked with a p) or part of the table's primary key (marked $p<n>, n$ an integer). A duplicate "key" is marked with a d; this indicates that this attribute is actually from another table and should not be included in size calculations for the current table. The fourth column, labeled "Screens," contains the one-letter abbreviation for the screen in which the attribute appears. The fifth column, labeled "\#," gives the sequential number of the data field with which the attribute is associated.

The sixth column contains the domain, the data type for the attribute. At the beginning of the file there are actually two columns labeled "Domain." The second one contains the alphabetically ordered listing of all of the unique domains. The sorting is performed within Lotus ㅇ $^{1-2-3} \otimes$.

4.1.3.2 SCREEN.PRN. Appendix B.2 contains a listing of the SCREEN.PRN file that is related to the above ENTITY.PRN file. Here the attributes are sorted on the basis of the screen with which they are associated, in order of increasing data field number. A change in the contents of the column "Screen" signals the start of a new screen. The columns are identical to those in ENTITY.PRN, except for the absence of the second "Domain" column; CREATE_SCHEMA, having read ENTITY.PRN before reading SCREEN.PRN, already knows the unique set of domains it will encounter.

The creation of this screen-sorted listing logically precedes the creation of the above entity-sorted listing. Within Lotus $\otimes 1-2-3 \otimes$, the screen-sorted listing can be sorted by entity and attribute to give the entity-sorted listing.

\subsubsection{Output Flles}

One output file from the CREATE_SCHEMA tool, CREATE_<name of the schemas.SQL, contains the SQL commands necessary to construct the specified database. A listing of the instruction set used to create the sample tracking database is found in Appendix D.

The remaining output files contain precompiled SQL statements to be included in the FORTRAN code for the user interface. Examples of code similar to that generated can be found above in Design of the User Interface, section 3. The main difference is in the naming of the scalar variables; the default 
names have been replace of Data, section 3.2.1.1) abbreviations that reflect the name of the associated attribute (see Storage 


\section{TOOLS USED IN DESIGNING THE USER INTERFACE}

\subsection{BUILD_SCREEN}

\subsubsection{Purpose of the Routine}

The data screens could be constructed using a series of VAX VMS screen management system routine calls, each of which contains the complete description for an individual data field. Or the calls could be executed in a loop, the descriptions for the fields coming from the appropriate arrays. Preferring to keep data separate from code within the user interface program, the latter method is used here. BUILD_SCREEN constructs the needed arrays, along with the associated declaration statements. BUILD_SCREEN is run once for each data screen, and a separate output file is created for each data screen. The output files for all of the data screens can then be concatenated together into a single field definitions file (FLDDEF.INC) that is INCLUDEd in the source code for the user interface.

BUILD_SCREEN does not ask any questions of the programmer, and so it can be executed as a spawned process. To execute it as a spawned process, enter, for example,

\section{SPAWN/NOWAIT @BUILD_SCREEN MAIN M Y}

at the DCL prompt (the user-specified parameters that follow @BUILD_SCREEN are described below under Flow Description of the Routine, section 5.1.2). To execute BUILD_SCREEN interactively, simply enter

\section{@BUILD_SCREEN MAIN M Y}

at the DCL prompt.

\subsubsection{Flow Description of the Routine}

The DCL code for BUILD_SCREEN appears in Appendix E. Again, there are no declaration statements in DCL; numbers can be stored only as integers, and character strings are distinguished from numbers by enclosing the value in quotation marks.

Up to three user-specified parameters can be included in the command line when invoking BUILD_SCREEN. The first, p1, identifies the input file. An input file should have a name of the form $<$ p1>_SCREEN.DAT; $<\mathrm{p} 1>$ could be MAIN for the main data screen, for instance. The second, p2, is a one-letter abbreviation for the data screen - $M$ for main. This letter is used in constructing the names of the arrays to ensure that unique names are created for any data screen. The third parameter, p3, is optional, and tells BUILD_SCREEN whether to include some declarations needed for the user interface but that are not directly related to any screen.

First, the input file is opened. This file contains a picture of how the data screen should look, minus the field renditions (normal text, bold, ... ). The programmer constructs the input file using a full screen editor; an example is presented in Appendix $F$. The numbers across the top and down the left-hand side are part of the file and are included for reference. There are 20 lines and 78 columns available for data fields; the remaining two columns and two of the remaining three lines are used to draw a box around the screen. The twenty-first line is used to display some reminders about common keystrokes.

Six temporary output files are then opened. FIELDS.DAT will contain the FORTRAN code for 
filling the arrays that will describe the data fields; declaration statements are not used to do this so that all of the information for a given field can be grouped together in one place. To SCRDEC.DAT will be written the FORTRAN declaration statements for the arrays, with sizes appropriate for the number of fields in the data screen. A short bit of code for setting initial default values for the field renditions will be written to DEFFLD.DAT. The last three files, CHARAC.DAT, LOGICA.DAT, and EQUIVA.DAT, will contain the scalar versions of the contents of the data field description, the logical-like if-null-indicator arrays, and the equivalence statements that connect the scalar variables to the appropriate array elements, respectively. Appropriate comments are written to these last three files immediately after they are opened.

After initializing counters for the line number currently being scanned for fields (LINMBR) and for the number of data fields found so far (NUMFLD), the input file is read one line at a time. The first line of the input file contains column numbers and so is not scanned for data fields. As each subsequent line is read, LINMBR is incremented by one. If a blank line is encountered in the input file, it is treated as a numbered line but not scanned for fields.

Each non-null line is scanned, one character at a time, for fields. The beginning of a field is signaled by an alphanumeric character (FIRSTC) must appear in column one, or in a later column (at most up to column 63 - MAXNFC) but preceded by a blank. The field's alphanumeric descriptor is expected to precede a series of underscores; the underscores indicate the maximum-allowed length for the data to be' entered.

The descriptor, FIELD, is all of the characters from the first one detected up to but not including the first underscore, minus any trailing blanks. The column in which a new data field description begins is saved in FDSCOL. From FDSCOL, the line is scanned until an underscore is encountered; the column in which the first underscore appears is saved in FINCOL. The length of the data entry field, MAXNOC, is the number of underscores found. With a completely described data field in hand, the number of data fields found, NUMFLD, is incremented by one, and the appropriate field-specific statements are written to the output files FIELDS.DAT, CHARAC.DAT, EQUIVA.DAT, and LOGICA.DAT.

Starting at the column after the last underscore, BUILD_SCREEN looks for another data field in the same line. If none are found, the next line is read and scanning starts again at column one. Once all of the lines have been scanned, the FORTRAN declaration statements for the various arrays can be written, along with the bit of code to initialize the field renditions. The six temporary output files are then concatenated into a single file, $\langle$ pl $>$ SCREEN.FOR. Appendix $G$ contains the single output file that results from the processing of the input file found in Appendix $F$. The temporary output files are deleted before the routine exits to the DCL prompt. The only hand-editing that needs to be performed on the output file involves specifying the field renditions to be used for each field.

Once BUILD_SCREEN is executed for all of the data screens, the individual output files can be concatenated together and rearranged into a single field definitions file, call it FLDDEF.INC. Appendix $\mathrm{H}$ contains the FLDDEF.INC file for the user interface to the sample tracking database. This file is INCLUDEd in the source code for the user interface. Keeping these definitions in a separate include file reduces the size of the file containing the rest of the source code for the user interface. For the sample tracking database, FLDDEF.INC takes up more lines than the rest of the source code. 


\section{SOME SOURCE CODES WITH BROAD APPLICATION IN DATABASE USER INTERFACES}

\subsection{For Use in Displaying Screens}

\subsubsection{DISPLY}

6.1.1.1 Purpose of the Routine. The source code for the FORTRAN subroutine DISPLY is presented in Appendix I. This routine makes use of VAX VMS screen management (SMG) system routines to display the various screens of the user interface on the user's monitor. In particular, these system routines allow the user interface to place text in the precise location desired on the user's monitor. Further, the text can be rendered in a number of ways: normal, bold, reverse video, and blinking.

The routine handles the initial data entry functions. It also confirms that the appropriate type of data has been entered into each field. It will flag those fields that are in error and keep the user from moving on to another screen until the errors are corrected.

6.1.1.2 Flow Description of the Routine. The calling routine passes to DISPLY all of the information needed to describe the fields to be displayed on the user's monitor. This includes what text is to be printed, the rendition in which it is to be printed, the type of data to be entered into each field, and any special relationships that exist between data fields. Declaration statements for the variables to contain this information follow, along with the necessary screen management system routine include files.

The first step is to assure that the virtual keyboard (where the user's input is coming from) is established correctly. This is done by first deleting any existing virtual keyboard (SMG\$DELETE_VIRTUAL_KEYBOARD), and then creating a new one (SMG\$CREATE_VIRTUAL_KEYBOARD). Once this is done the various fields can be displayed.

Calls to SMG\$PUT_CHARS place the fields in their proper locations. The renditions used are field-dependent: if an entry in this field is required, render the field in bold; if the entry is already fixed and cannot be changed, render the field in reverse video. (These renditions are specified by hand-editing the output file from BUILD_SCREEN. See Flow Description of the Routine, section 5.1.2.) All data input fields are rendered as underlined (FINPUT); this makes the screen look like a fill-in-the-blank form. Additional calls to SMG\$PUT_CHARS add comments and key stroke reminders.

Beginning at the field IBEGIN, DISPLY waits for user input. This may be directives for moving the cursor (such as the arrow keys), or data entered into a data field. All input is accepted via SMG\$READ_STRING and then interpreted appropriately. DISPLY directly handles the special function keys PF1 (for aborting execution), PF2 or Help (for displaying the special function key mapping via the KEYPAD routine - see Keypad, section 6.1.4), and "Insert Here" to ditto data from a connected field into the current field.

Other special function keys are acted upon after the contents of the current data field are checked against the requirements for the kind of information needed in that field. Dates should be given as mmddyy (all integers); numbers can be real, integer, or exponential; and so on. If the type of information given is not correct, DISPLY marks the field as being in error by setting its attribute as blinking in addition to the original setting. The user must correct the errant data before DISPLY will move on to another field. Once the error is corrected, DISPLY retums the rendition of the field to its original setting. 
Once valid data is entered into a field, DISPLY calls the routine TRMNTR (see TRMNTR, sect 6.1.2) for any further action on the keystroke used to terminate the data field entry. If the user sign: the interface that there are no more data fields to be filled in (by striking "Do" or ctrl-Z), DISPLY sca. through all of the fields on the screen for fields left blank that need to be filled in. Fields that need wa be filled in are treated as if they contained errant data (see above), and the user must again strike "Do" or ctrl- $Z$ after having filled in the field correctly. After all of the required fields are filled in and any errant data corrected, DISPLY renews the virtual keyboard (as explained above) before returning to the calling routine.

If the user has requested that the cursor move to another field, TRMNTR returns the number of the next possibly accessible field. DISPLY then starts at one field before the field retumed by TRMNTR and moves ahead one field at a time until an accessible (i.e., non-fixed) field is found. Note that when the initial field IBEGIN is passed from the calling routine, DISPLY decrements it by one before starting to look for the next accessible field.

\subsubsection{TRMNTR}

6.1.2.1 Purpose of the Routine. The source code for this FORTRAN subroutine is present in Appendix J. TRMNTR acts on the terminator received by SMG\$READ_STRING; recall th: SMG\$READ_STRING is used in DISPLY to read user input to a data screen.

6.1.2.2 Flow Description of the Routine. The calling routine passes to TRMNTR most of the information needed to describe the fields to be displayed on the user's monitor. This includes what text is to be printed, the rendition in which it is to be printed, and where it is to be printed. Declaration statements for the variables to contain this information follow, along with the necessary screen management system routine include files.

Some of the actions taken by TRMNTR have no immediate impact. For example, if the user strikes PF4 (Advance) or PF5 (Reverse), TRMNTR remembers that the user has specified a direction to be used with any subsequent "Next" keypad keys (all of which move the cursor to the next field). TRMNTR acts on most of the other terminators to specify the next field to which DISPLY should try to move. For example, striking the up-arrow key causes TRMNTR to scan backwards through the locations of the fields until it reaches one which is on a line above the current field. TRMNTR is set up to treat the data screen as a sphere; for example, striking the down-arrow key from the bottom line initially moves the cursor to the top line.

Other actions performed by TRMNTR include those special cases DISPLY handles directly (the main routine also calls TRMNTR, for example). It can also erase the contents of a data field when directed to do so by either "Remove" or one of the "Delete" keypad keys. And if it does not recognize the terminator, TRMNTR does not move the cursor. Note that the tab key is not a terminator. The VMS default set of terminators recognized by the screen management routines does not include the tab key. The programmer can construct a set of terminators that includes the tab key for use in the SMG\$READ_STRING calls.

\subsubsection{CHOOSE}

6.1.3.1 Purpose of the Routine. The source code for the FORTRAN subroutine CHOOSE is presented in Appendix $\mathrm{K}$. This routine makes use of VAX VMS screen management system routine: to overlay a second screen on top of the data entry screen. This overlaid screen provides the user witr: some choices for a field (or series of fields) to be filled in. For this user interface, CHOOSE is used to 
display a list of known sample possessors to speed the updating of sample entries. It performs as a scaleddown version of DISPLY, calling TRMNTR as is needed.

6.1.3.2 Flow Description of the Routine. The calling routine passes to CHOOSE all of the information needed to describe the fields to be displayed in the overlaid screen. Declaration statements for the variables to contain this information follow, along with the necessary screen management system routine include files.

After creating (SMG\$CREATE_VIRTUAL_DISPLAY) and pasting (SMG\$PASTE_VIRTUAL_DISPLAY) the overlaid screen, CHOOSE renews the virtual keyboard as does DISPLY. It then displays the list, and waits for the user to either select the current item in the list or move to another. To select an item in the list, the user need only put some character in the corresponding field. Once the user strikes "Do" or ctrl-Z, CHOOSE determines which item, if any, the user selected by searching for the first field filled in. The number of the item selected is returned to the calling routine; the calling routine then fills in the field(s) appropriately.

Before returning to the calling routine, CHOOSE removes the overlaid screen (SMG\$ERASE_DISPLAY, followed by SMG\$UNPASTE_VIRTUAL_DISPLAY) and, once again, renews the virtual keyboard.

\subsubsection{KEYPAD}

6.1.4.1 Purpose of the Routine. The source code for the FORTRAN subroutine KEYPAD is presented in Appendix L. This routine makes use of VAX VMS screen management system routines to display the mappings of the special function and keypad keys. This mapping is presented as an overlaid screen, much as CHOOSE does.

6.1.4.2 Flow Description of the Routine. The calling routine passes no arguments to KEYPAD. Declaration statements for the variables to contain the information needed to describe the overlaid screen follow, along with the necessary screen management system routine include files. KEYPAD then creates and pastes into place the overlaid screen.

As with DISPLY, TRMNTR, and CHOOSE, KEYPAD renews the virtual keypad. KEYPAD removes the overlaid screen as soon as any key is struck. TRMNTR is not called, nor does KEYPAD handle any special function keys itself. After removing the overlaid screen, KEYPAD renews the virtual keyboard and returns to the calling routine.

\subsection{For Use in Data Type Conversions}

\subsubsection{Character String-to-Numerical Value Conversions: RVALUE}

6.2.1.1 Purpose of the Function. The source code for the FORTRAN real function RVALUE is presented in Appendix M. This function attempts to convert the data represented in the character string passed to it into a real number. The converted data is for insertion into the database. The data can represent an integer, real, or exponential number.

6.2.1.2 Flow Description of the Function. After declaring the few variables needed, RVALUE determines whether the data represents an integer by looking for a decimal point. If it does not 
find a decimal point, the characters passed to it are assumed to represent an integer. Since DISPLY has already confirme that the corresponding field does contain something that looks like a number, a failure of the intemal read used to effect the conversion is assumed to be falal to further execution. Once the internal read into an integer variable is performed, RVALUE converts the integer value to a real value, then exits.

If a decimal point is found, an internal read is performed to obtain the real number directly. Note that the format used for the internal read works for real numbers in exponential notation as well as for real numbers without an exponent.

\subsubsection{Numerical-to-Character String Representation Conversions: CVALUE}

6.2.2.1 Purpose of the Function. The source code for this FORTRAN function, the functional inverse of RVALUE, is presented in Appendix N. As the inverse of RVALUE, CVALUE attempts to convert the number passed to it into a character representation; the conversion of ateger values is performed in calling sontine since only an internal wrte is req: ad. The c. acter representation is for us : displayin. he data from the database using scree anagemen stem routines.

A separate routine was prepared to handle real numbers so that the conversion effected (using FORTRAN F or E format) would generate the most complete character representation that will fit in the allowed field. For example, consider a field that is only eight characters long. The number to be presented in that field is 0.000000123 . Using an F format, the character representation would be 0.000000 - likely not a very good representation. If the leading zero were dropped, the F format would only allow for .0000001 . But if an $E$ format was used, the representation would be $1.23 E 07$, a much more complete representation.

6.2.2.2 Flow Description of the Function. Following the few variable declarations needed, CVALUE first tries to represent the number using an $F$ format. The first $F$ format attempted places as many digits as possible after the decimal point. As in the above example, this is generally the best $F$ format to begin with

If an error occurs during the internal write, it likely means that there are not enough digits before the decimal point to represent the number. CVALUE then effectively moves one character from after the decimal point to before it by respecifying the $\mathrm{F}$ format. If an error again occurs during the internal write, another character is moved. This will continue until a successful-internal write is performed or there are no more characters to be moved (in which case an $\mathrm{E}$ format will surely be needed).

If an internal write using an F format is successful, CVALUE then calls RVALUE to obtain the real number represented by the conversion. (It is possible that the real number represented by the conversion does not have the same value as the original real number.) CVALUE then attempts to generate the best $\mathrm{E}$ format representation in a fashion identical to that used for generating the best $\mathrm{F}$ format representation. Once a successful internal write is achieved using an $E$ format representation, the real number corresponding to the representation is again obtained using RVALUE (i.e., a back translation is performed).

Finally CVALUE decides of the conversions failed (i.e., none the other conversion. If both cony conversions succeeded, CVALUE co: presentation should be retumed to the calling routine. If one nternal writes succeeded), then CVALUE returns the result of is fail, the character representation returned is 0.0 . If both res the results of the two back translations to the original value. 
The back-translated value closest to the original value is the one returned to the calling routine.

\subsubsection{Conversions between mmddyy Dates and Integer Date Counts}

6.2.3.1 Purpose of the Functions. The source code for the FORTRAN functions DAYCNT and CHRDAT are presented in Appendix $O$. These functions are used to convert dates of the integer form mmddyy to the day count from January 1, 1990 (day count 1) and vise versa.

6.2.3.2 Flow Description of the Routine. A small number of variables are first declared in both functions. Then the appropriate mappings are performed.

DAYCNT uses the following FORTRAN expression to directly relate an mmddyy date to a day count:

$$
\text { daycnt }=(\text { year-1990)*365 }+ \text { int }((\text { year-1989)/4) }+ \text { edamnt }(m m)+\text { dd, }
$$

where the first term gives the number of days from fully elapsed years since 1990 , the second term corrects for leap years, the second term represents an array (EDAMNT) that contains the number of days fully elapsed up to the mm-th month, and the fourth term is simply the date of the month. If yy is less than 90, the date is assumed to be in the 2000s; otherwise the date is assumed to be in the $1900 \mathrm{~s}$, and year is determined appropriately. The leap year correction is valid for the next $100+$ years; recall that years evenly divisible by 100 are not leap years unless they are also evenly divisible by 400 , which the year 2000 is.

CHRDAT, the inverse of DAYCNT, does not directly obtain the mmddyy date from the day count. First it makes a rough guess at the year by dividing the day count sent to it by 365 . Then, based on the rough guess, CHRDAT corrects for the number of leap years that have elapsed since 1990 to determine the correct year. With the correct year in hand, CHRDAT calculates how many days remain after all of the fully elapsed years are subtracted from the total. The month is determined by subtracting the number of days in each month from the remnant; once a negative value is obtained, CHRDAT knows that the correct month is the prior one. The final remnant is the date of the month.

\subsection{For Use in Constructing Searches}

\subsubsection{SRCBLD}

6.3.1.1 Purpose of the Routine. The source code for the FORTRAN subroutine SRCBLD is presented in Appendix P. Given the variability of searches, and that the user interface cannot know a user-specified search before it is constructed, the appropriate SQL call needs to be prepared on the fly and executed as dynamic SQL. SRCBLD constructs the SQL call, compiling the attribute and domain names and the associated values into a character string (via APSTRG) with as few extra blanks as possible. The current limit for the length of the character string is 6000 characters, split evenly between the "WHERE" clause and the rest of the cursor declaration call.

6.3.1.2 Flow Description of the Routine. The calling routine passes to SRCBLD the values of all of the fields for a data entry screen, along with the array containing the name of the appropriate attribute(s) and domains (ATRBUT). These variables are all defined in the declaration statements, along with the two pieces of the character string. 
SRCBLD the ns through all of the fields to find out which ones have been filled in. Only those that have been fil in are included in the SQL call that performs the search. Once a filled-in field is found, SRCBLD add e name of the appropriate attribute to the "FROM" clause of the SQL call. The "FROM" clause is fov at the end of the first piece of the character string. For the sample tracking database, the domain SAMPLE_TRACKING_ID serves as the primary or foreign key for the various database files. To ensure that the search finds only those entries for which all of the specified information applies fully to each entry, SRCBLD automatically includes the condition that all of the SAMPLE_TRACKING_IDs from a single successful hit match. Again, the actual tacking on of the attribute name to the existing string takes place in the subroutine APSTRG (see APSTRG, section 6.3.2).

The attribute, domain, and field value are then all added to the second piece of the character string in the form <attribute>.<domain>=<field value>. This version of SRCBLD only allows for exact equalities; wildcards, ranges, and "sort of like"s have not yet been implemented.

\subsubsection{APSTRG}

6.3.2.1 Purpose of the Routine. The source code for this FOF $N$ subroutine esented in Appendix Q. APSTRG "appends" new character strings to an existing "Appends" I: totally accurate, though; APSTRG does not perform a concatenation. Rather, APS $\quad s$ keeps track $\varnothing \quad v$ many characters are in the existing string, and then puts the contents of the new string in after that This requires that the existing string have enough room for all of the new strings to be included within it.

6.3.2.2 Flow Descriptlon of the Routine. The calling routine passes to APSTRG the existing character string, the number of characters in the existing string, the new string to be added, and a flag that tells APSTRG whether the existing string should be started afresh and so contain only the new string upon return to the calling routine. These variables are all defined in the declaration statements.

If the existing string is to be started afresh, APSTRG starts by resetting the number of characters filled in in the existing string to zero. APSTRG then assumes that all of the characters passed in the new string are to be included in the existing one. Since the length of the new string can vary, APSTRG determines this length using the FORTRAN intrinsic function LEN. The new string is then copied into the existing one, the length of the new string added to the prior length of the existing string, and thon APSTRG returns to the calling routine. 


\section{REFERENCES}

1. D. A. Femec, User's and Reference Guide to the INEL RML/Analytical Radiochemistry Sample Tracking Database Version 1.00, INEL-95/0455, September 1995.

2. This document. 


\section{APPENDIXES}

\section{A. CREATE SCHEMA - A ROUTINE FOR CONSTRUCTING AN INSTRÚCTION SET FOR CREATING A DATABASE}

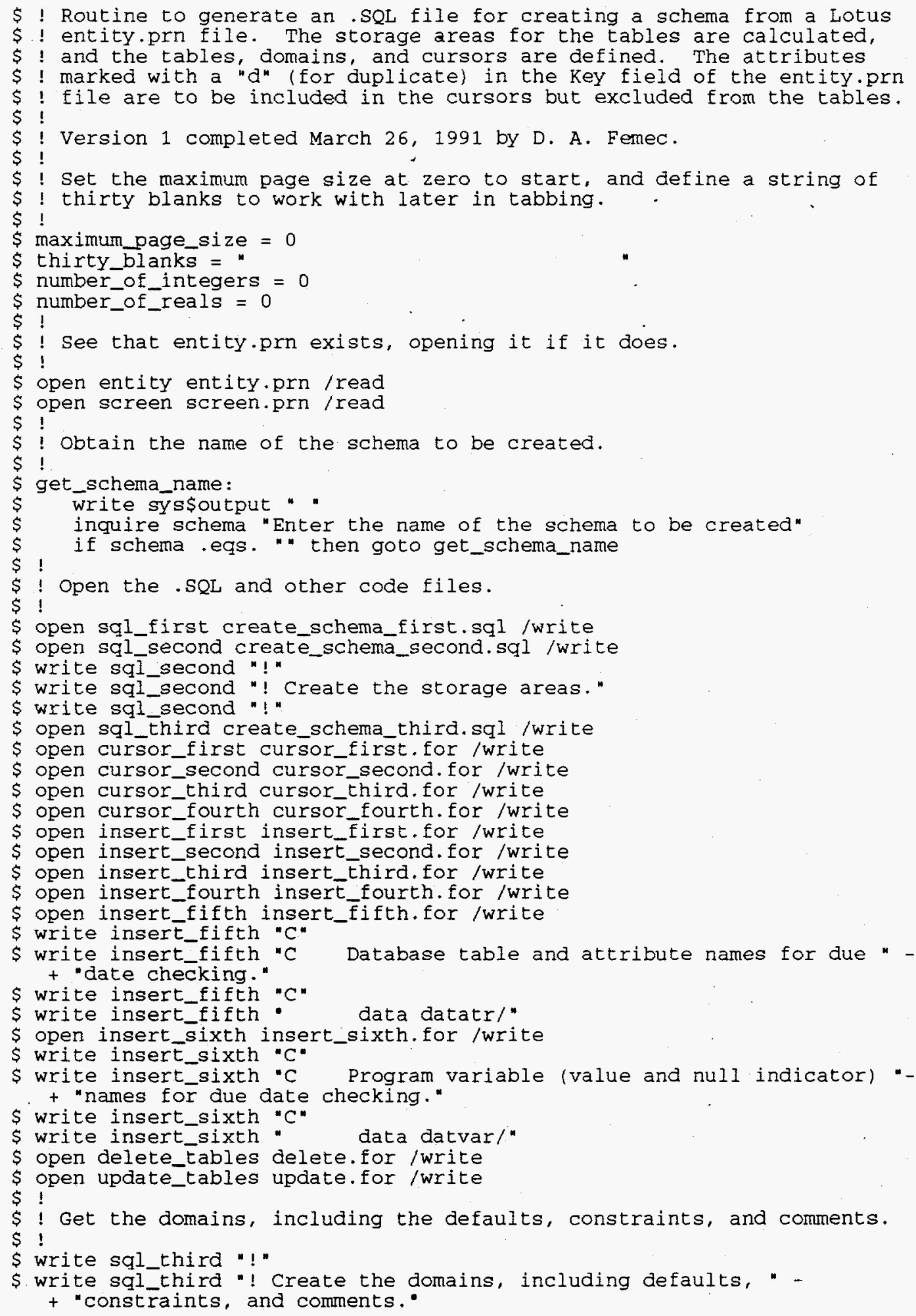




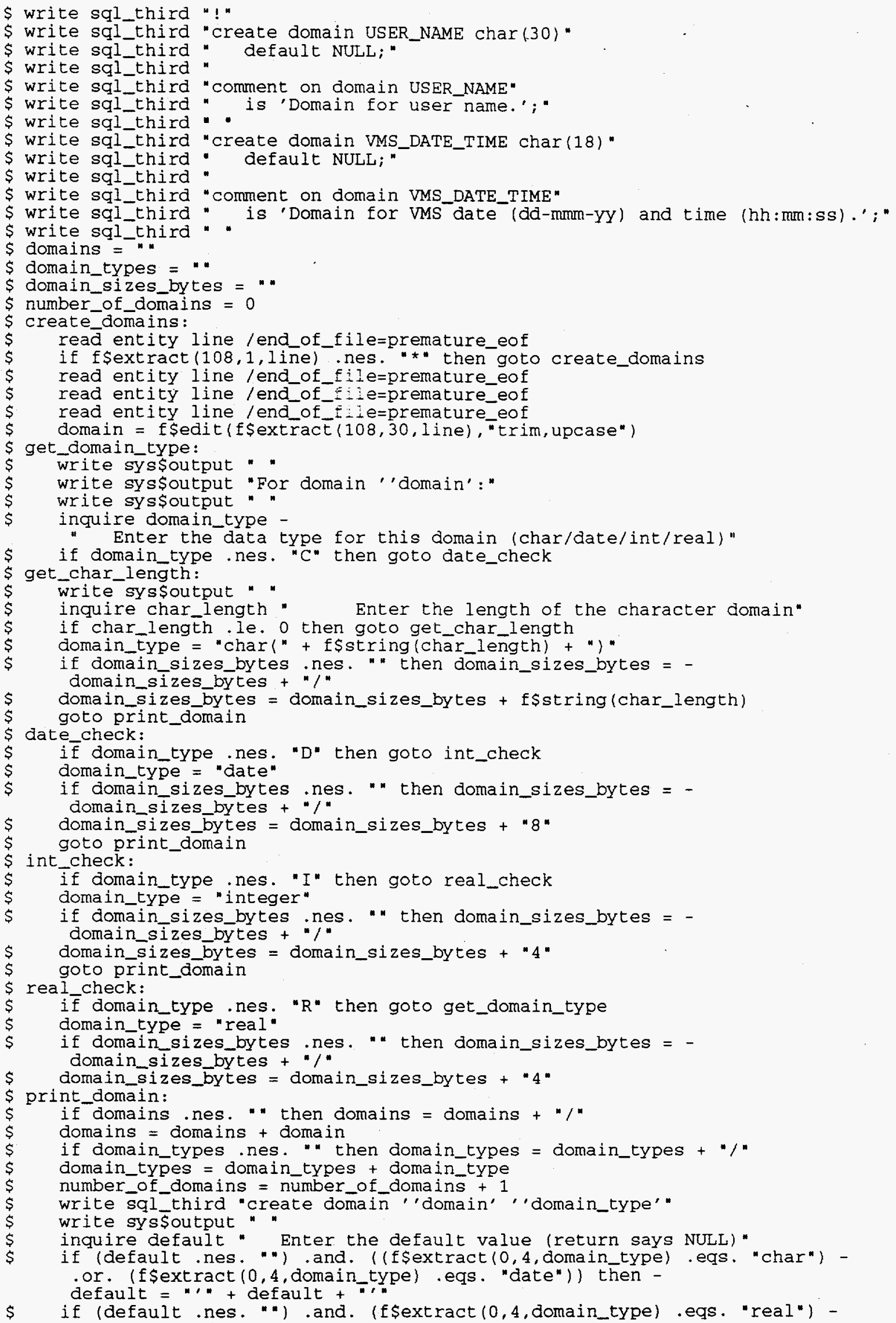




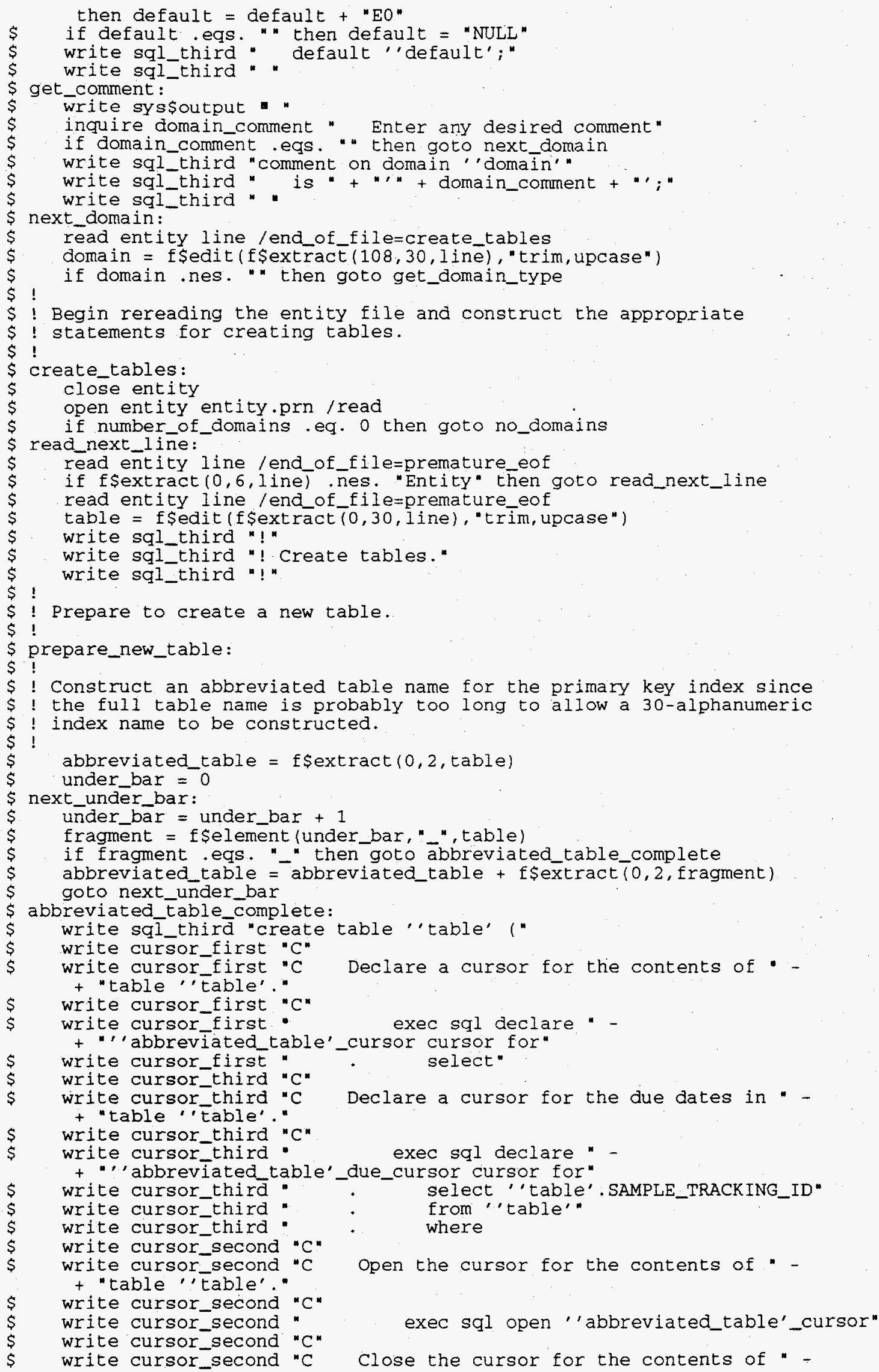




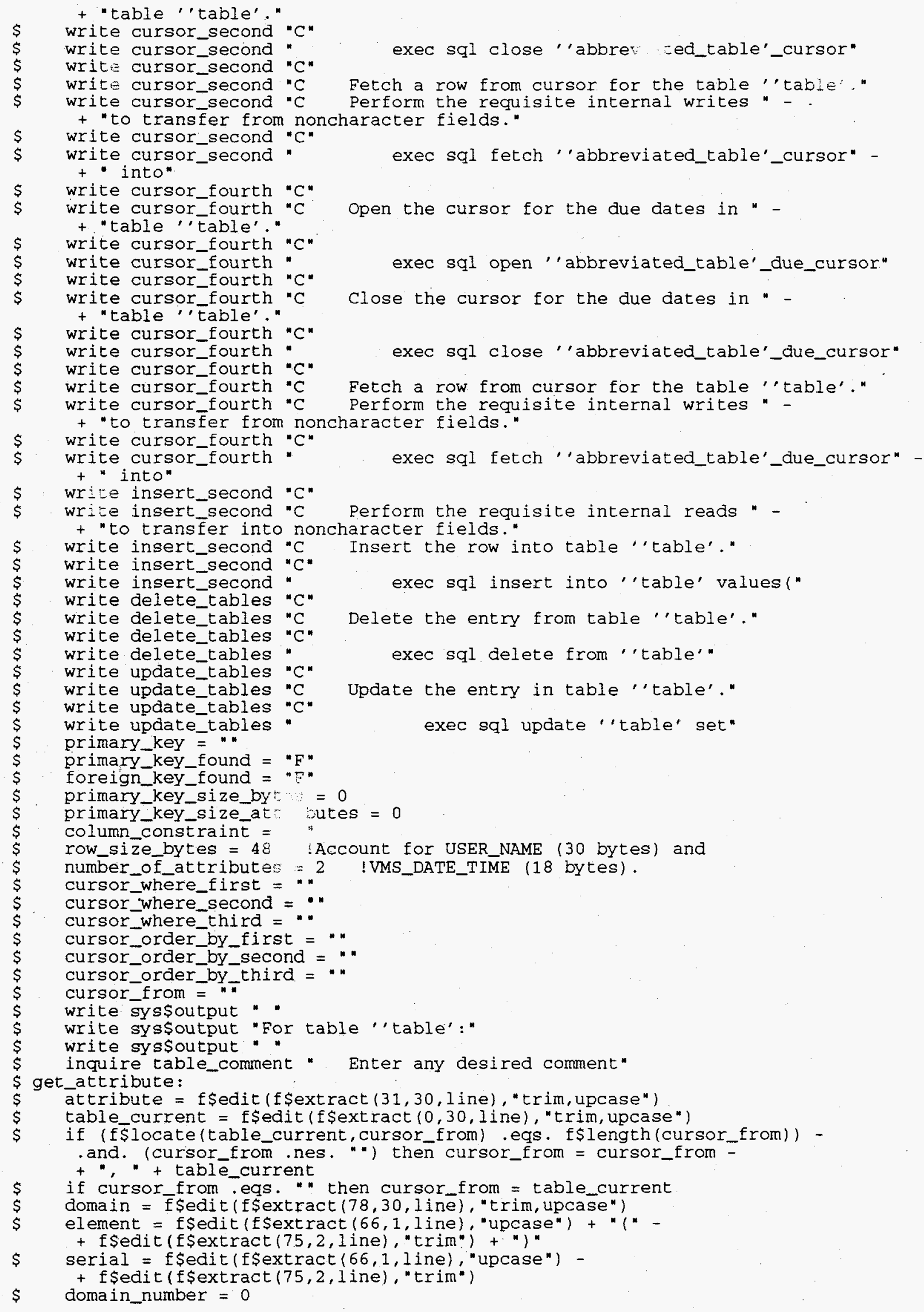




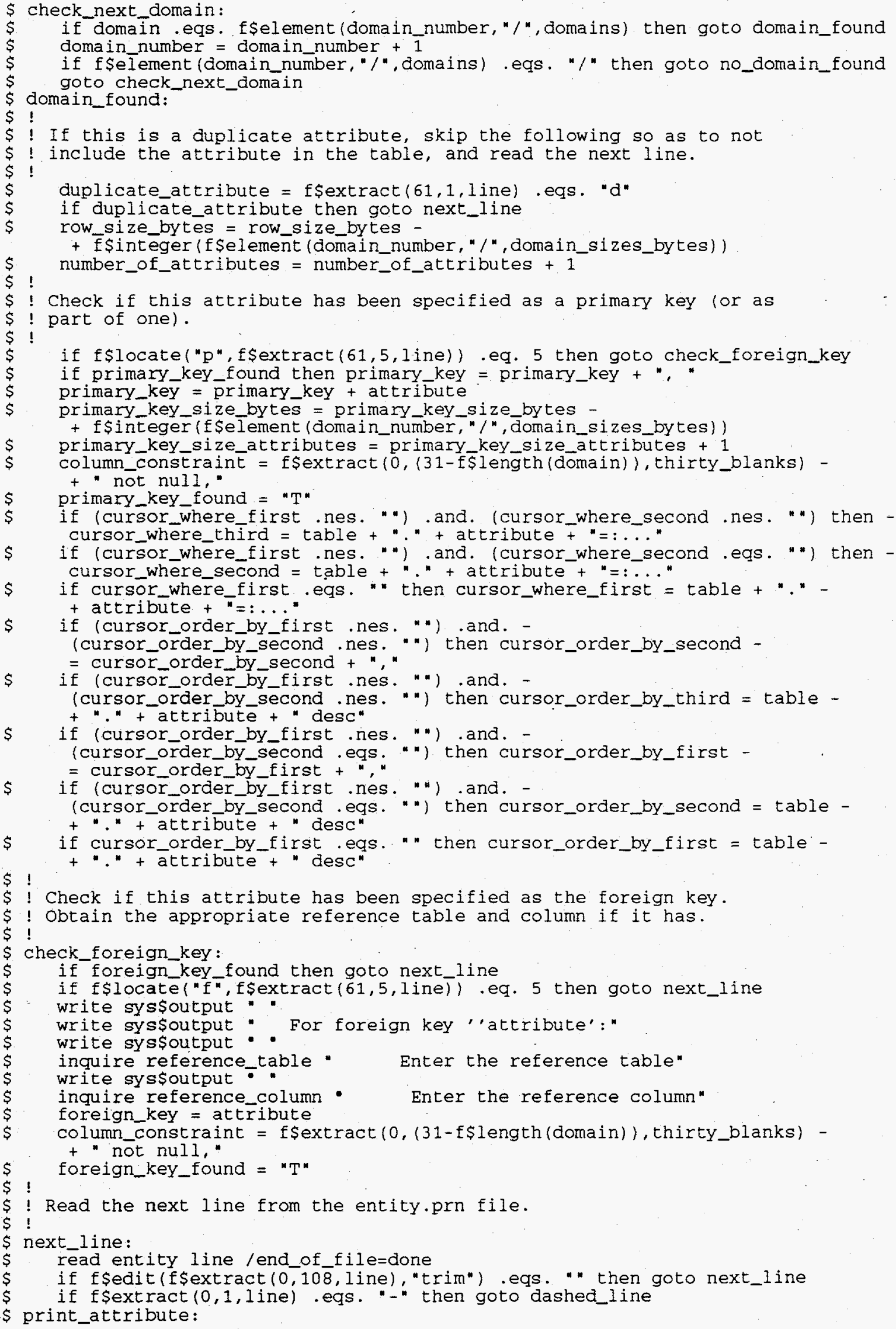




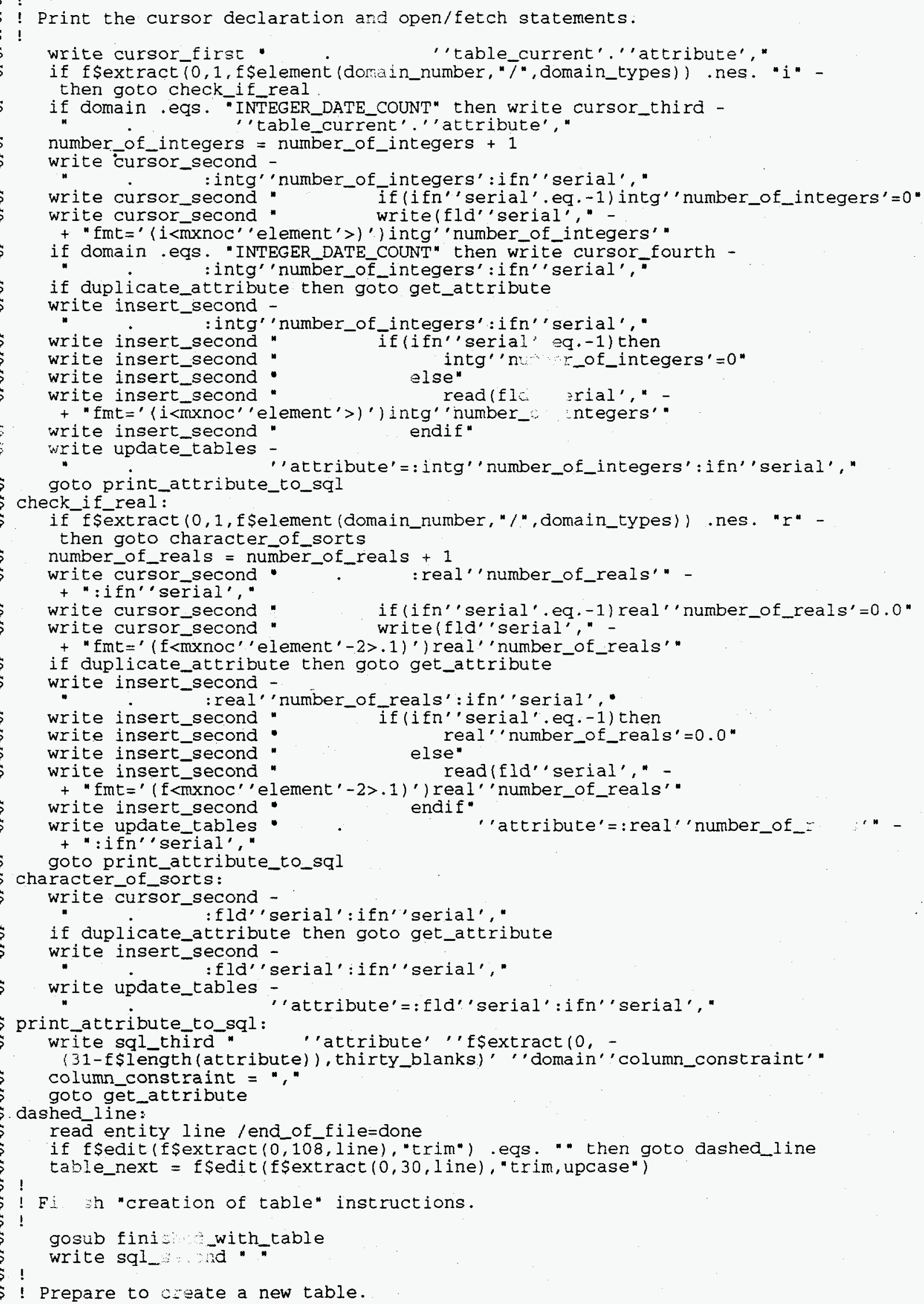




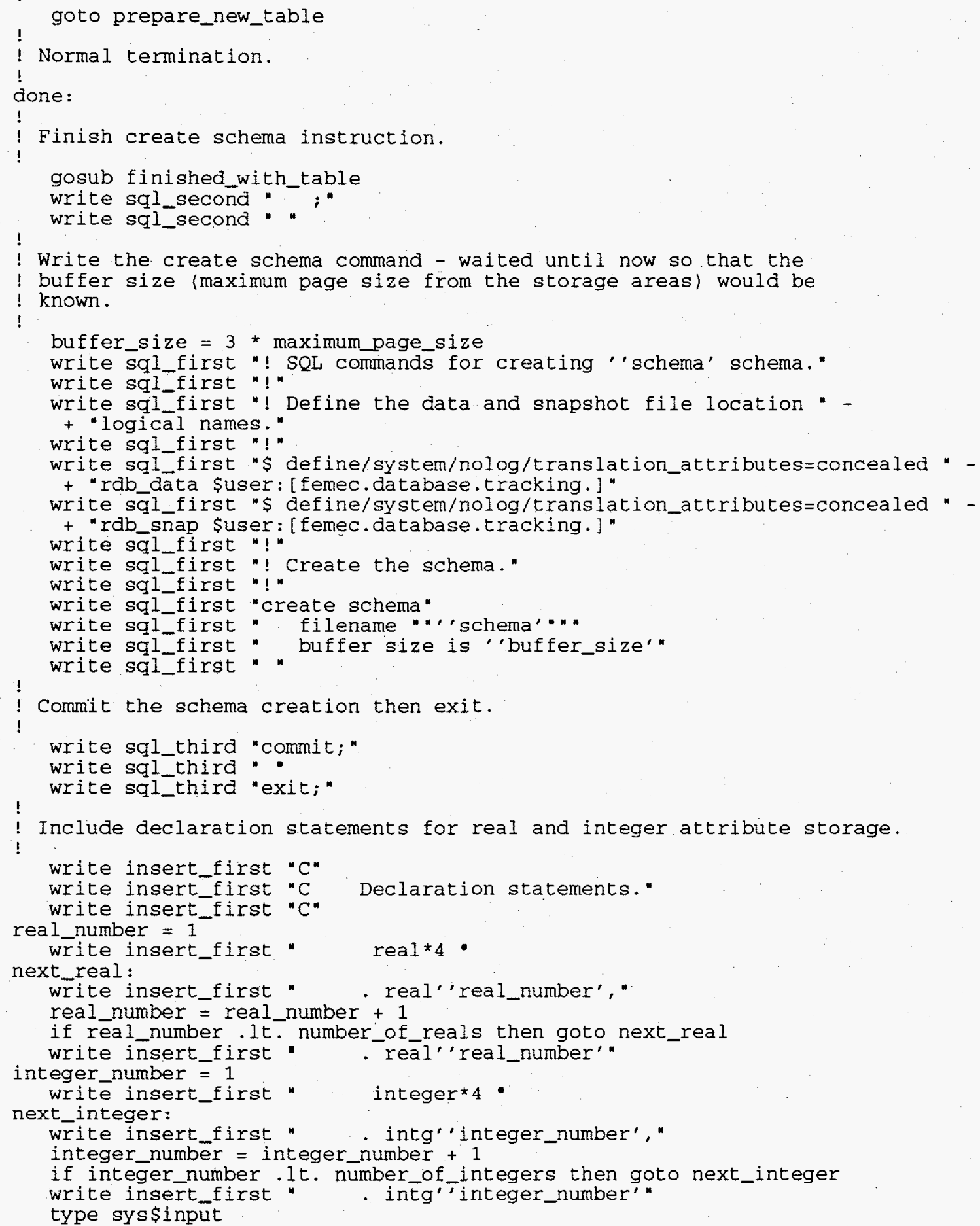

Execution has successfully completed. The create_schema.sql and cursor and insert files will be closed and retained. The entity and screen files will also be closed and retained.

Please wait while certain sections of the cursor and insert files are rearranged; this may take a few minutes.

close sql_first

close sql_second

close sql_third

copy/concatenate create_schema_first.sql + create_schema_second. sql - 


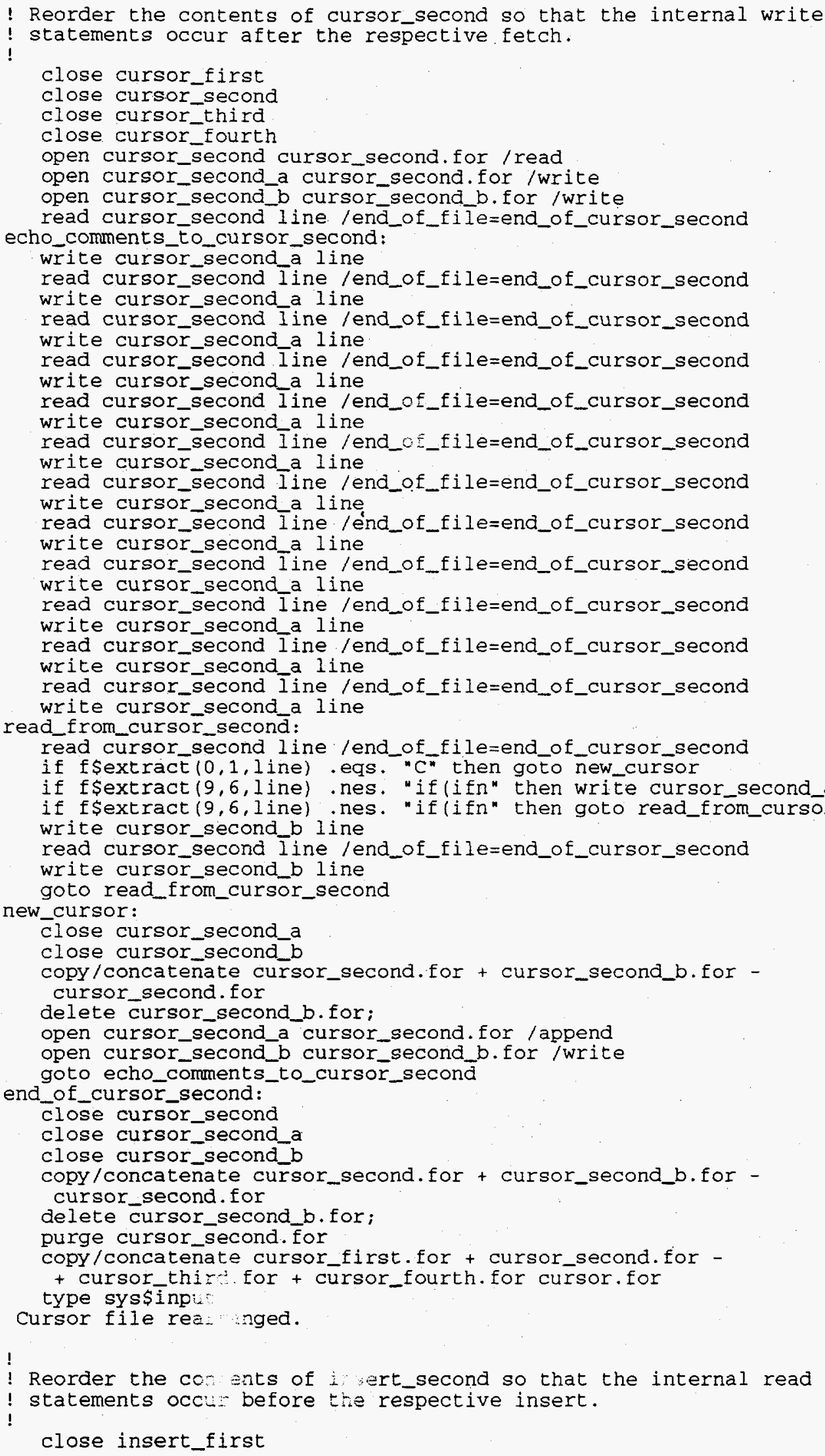

+ create_schema_third.sql create_'schema.sql 


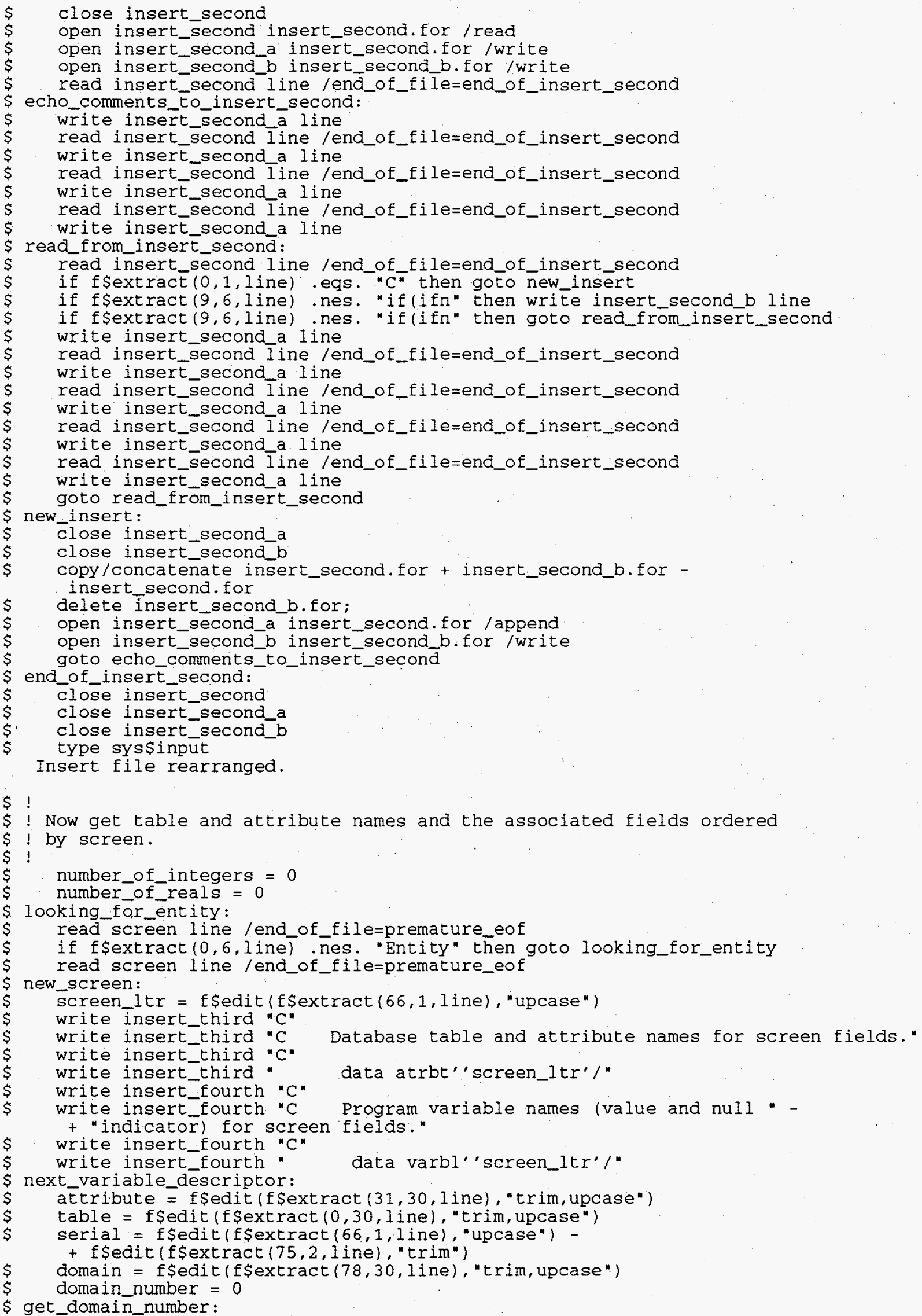


if domain .eqs, f\$element (dmain_.mber, " " anains) then goto get_next_line

doma in_number = domain_numbe $=+1$

if $\mathrm{f}$ selement (domain_number, "/",domains) .eqs. "/" then goto no_domain_found

goto get_domain_number

get_next_line:

read screen line/end_of_file=done_with_screen

if $\mathrm{f}$ \$edit (f\$extract $(0,10 \overline{8}$, line), "trim") .eqs. " then goto get_next_line

if $\mathrm{f}$ Sedit (f\$extract $(66,1,1$ ine), "upcase") .nes. screen_ltr then -

goto next screen

write insert third " ." + table + "' " + attribute + " "

if $\mathrm{f}$ Sextract $(0,1$, f \$element (domain_number, "/",domain_types)) .nes. "i" -

then goto is_it_real

number_of_intégers $=$ number_of_integers +1

write insert_fourth " ':intg' 'number of integers': ifn' 'serial'", "

if domain .nes. "INTEGER_DATE_COUNT" then goto next_variable_descriptor

write insert_fifth " -." + table + "," + attribute + ".,"

write insert_sixth " . ':intg' 'number_of_integers':ifn' serial" "." goto next_variable_descriptor

is it real:

if f sextract 10,1, f selement (c)

then goto must_be_character

number_of_reals = number_of

write insert_fourth "

goto next_variable_descriptor

must_be_character:

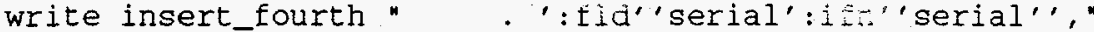

goto next_variable_descriptor

next screen:

write insert_third " . " + table + "'," + attribute + "/"

if $f$ sextract $(0,1$, fselement (domain number, "/" domain types)) .nes. "i" then goto is_this_real.

number_of_integers $=$ number_of_integers +1

write insert fourth " ': intg' number of integers': ifn' 'serial'"/"

if domain . nes. "INTEGER_DATE_COUNT" then goto new_screen

write insert fifth"

write insert_sixth ". . 'intg' number_of_integers':ifn' serial"',"

goto new_screen

is_this_real:

if $\mathrm{f}$ Sextract $(0,1$, f Selement (domain_number, "/",domain_types)) .nes. "r" then goto this_is_character

number_of_reals $=$ number_of_reals +1

write insert fourth " - ':real' number of reals':ifn' serial" /"

goto new_screen

this is character:

write insert_fourth " . 'fld' serial':ifn'"serial"'/"

goto new_screen

done_with_screen:

write insert_third ". . " + " "table'" + "'," + " "'attribute' /"

if f Sextract $(0,1$, f \$element (domain_number, "/",domain_types)) .nes. "i" then goto is_this_real_last

number of integers $=$ number of integers +1

write insert_fourth " .' intg' 'number_of_integers':ifn' serial" /" if domain .nes. "INTEGER_DATE_COUNT" then goto close_inserts

write insert_fifth " . " " table + " " " + attribute + " " "

write insert_sixth " : ': intg' number_of_integers':ifn' 'serial" /"

goto close inserts

is_this_real_last:

if f̧̄extract $(0,1$, f Selement (domain number, " " domain types)) .nes. "r" then goto this_is_character_last

$\$$ number_of_reals = number_of_reals +1

write insert_fourth " . 'real' number_of_reals':ifn' 'serial' /" goto close_inserts

this_is_character_last: write insert_fourth " .:fld' 'serial':ifn''serial'"/"

close inserts:

ose insert_third

ase insert_fourth

ciose insert_fifth

close insert_sixth

copy/concatenate insert_second.for + insert_second_b.for -

insert_second. for

\$ delete insert_second_b.for; 


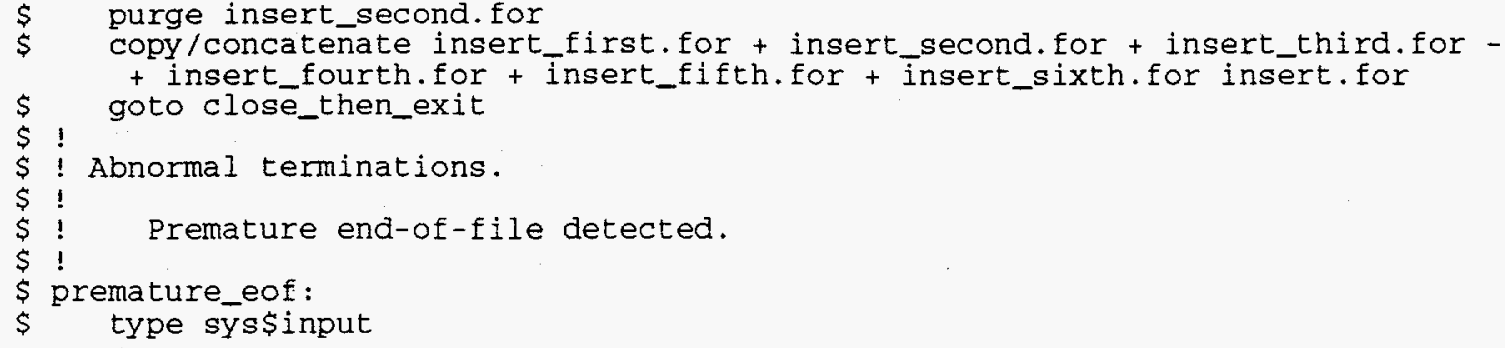

A premature end-of-file was detected. The create_schema.sql file being created will be deleted. The entity input file will be closed but not deleted.

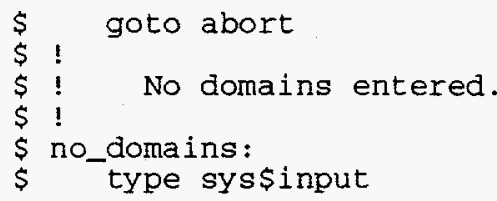

No domains were entered, hence no attributes can be created. The create_schema.sql and cursor and insert files being created will be deleted. The entity input file will be closed but not deleted.

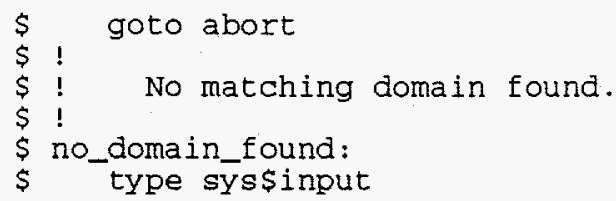

No matching domain was found for the current attribute. The create_schema.sql and cursor and insert files being created will be deleted. The entity input file will be closed but not deleted. 


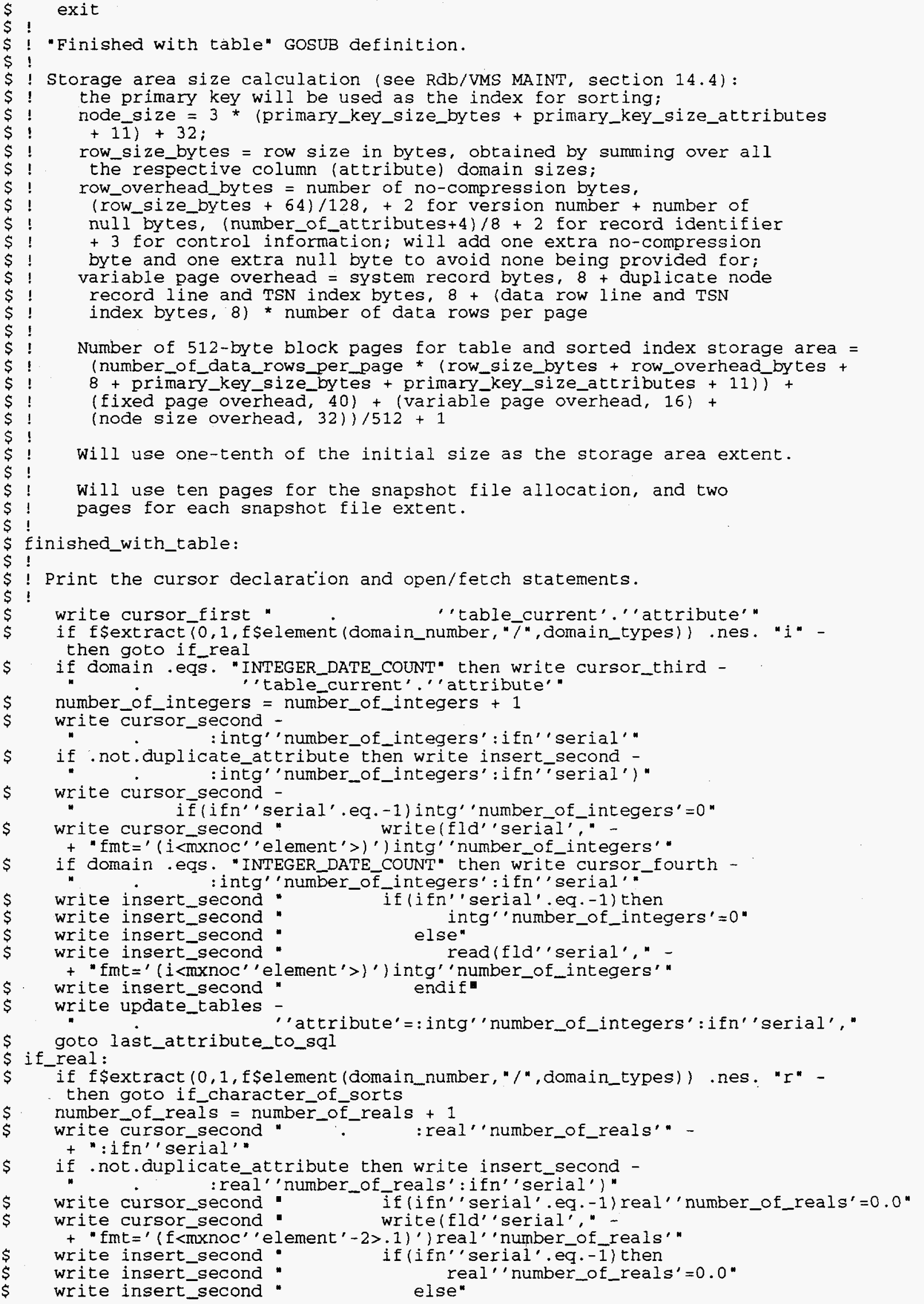


write insert_second

read (fld' 'serial'," -

+ "fmt $=$ ' ( $\mathrm{f}<\mathrm{m}$ xnoc' 'el

write insert_second "

write update_tables

real" number of reals' "

+ " ifn' serial',"

endif*

goto last_attribute_to_sql

$\$$ if_character_of_sorts:

write cursor_second -

". :fld' 'serial': ifn' 'serial'"

$\$$. if not.duplicate_attribute then write insert_second -

write update_tables

: ifn'serial',"

" attribute to sql:

$\$$ last_attribute_to_sql:
$\$$ write update tables

write update_tables - 


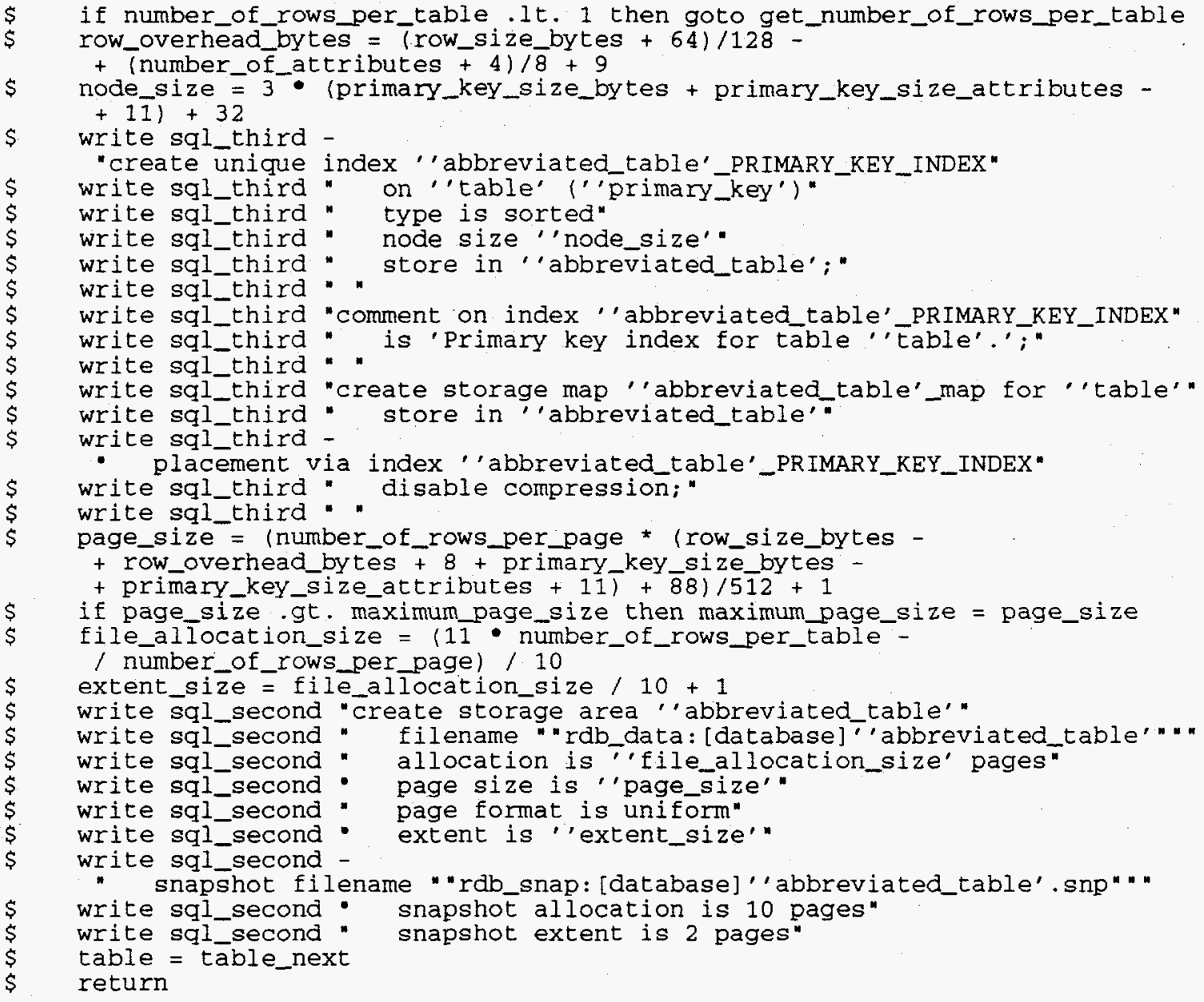


sample_information sample information sample information sample information sample informat ion sample information sample_information sample_information sample information sample_information alpha_uranium need_beta_st ront ium_89_and_90 . need beta other

need al pha uranium 作 need_alpha_plutonium. need_alpha_am241 sans pu238 need_alpha_am241_with_pu238 need_alpha_total_spectrometric need_alpha_other

sample_tracking_id
11 flags

19 flags

27 flags

35 flags

8 flags

13 flags

18 flags

23 flags

28 flags
33 flags

1 identification_codes sample_information

alpha_uranium

alpha_uranium

- -

sample information

alpha_thorium

alpha_thorium

alpha_thorium

alpha_plutonium.

cample_informat io

alpha_plutonium

alpha_plutonium

alpha_plutonium

alpha_am241_sans_pu238

sample_information

alpha_am241_sans_pu238

alpha 241 cans

alpha am241 sans pu238

alpha_am241_with_pu238

sample_information

alpha_am241_with_pu238

alpha_am241_with_pu238

a pha_am241_with_pu238

alpha_total_spectrometric

sample information

alpha total spectrometric

alpha total spectrometric

alpha_total_spectrometric

alpha_total_spectrometric

alpha_other

sample_informat ion

alpha_other

alpha other

alpha_other
- cust omer_sample_name results_needed_by_date _ate_all_work_completed results report citation

. sample_tracking_id

customer sample name

results_needed_by_date date_all_work_completed any_special_instructions

. sample_tracking_id

result needed name date all work_completed results report citation any_special_instructions

.sample_tracking_id

- customer_sample_name

results_needed_by_date results report citation any special instruct ions

. sample_tracking_id

customer_sample_name

results_needed_by_dace

date_all_work_completed results_report_citation

\section{sample_tracking_id}

customer_sample_name

results needed_by_date results report citation any_special_inst ructions

sample_tracking_id

cust omer sample name

date ali work by date

results report citation

any_special instructions d 4 integer_date_count 5 integer date_count 6 twenty_characters

1 identification_codes 2 sixty_characters 9 integer_date_count 10 integer_date_count

11 twenty_characters

\section{2 flage}

$\mathrm{f} / \mathrm{p}$ a 1 identification_codes 2 sixty_characters

14 integer_date_count

16 twenty characters

17 flags

$f / p$ a 1 identification_codes 2 sixty_characters 19 integer_date_count 21 integer date count 21 twenty

$\mathrm{f} / \mathrm{p} a=1$ identification_codes 2 sixty_characters 24 integer_date_coun 25 integer_date_count 26 twenty_characters

$f / p \quad a \quad 1$ identification_codes 2 sixty_characters 30 integer date count 31 twenty_characters 32 flags

$f / p$ a $\quad 1$ identification_codes 2 sixty_characters

34 integer_date_count

36 twenty characters

37 flags 
beta_stront ium_90

beta_stront lum_90

beta st ront ium 90

beta stront ium 90

beta st ront ium 90

beta_stront ium_90

beta_st ront ium_90

beta_st ront ium_89_and_90

sample_information sample_informat io sample_tracking_id

detocter sample name

detector_count_length_units

results_needed_by_date

results_needed_by_time results report citation

sample_tracking_id any_special_instructions

.customer_sample_name
$f / p b \quad 1$ identification_codes sixty_characters 4 real_number
6 integ
7 integer_date_count 8 integer_date count 9 twenty_characters 10 flags
$f / p \quad b \quad 1$ identification_codes 2 sixty_characters

12 real_numbers

13 units

14 integer_date_count

15 character_time_hh_mm

17 integer date_count

18 flags

detector_count_length_units

results_needed_by_time date_all_work_completed any special instructions

beta stront ium 89 and 90

. sample_tracking_id

beta_total_strontium

detector_count_length

detector_count_length_units

results_needed_by_date

results_needed_by_t ime

dace all work any special instructions

beta_total_stront ium

beta total stront ium

beta_trit ium
sample_information

beta_tritium

beta_tritium

beta_tritium

beta tritium

beta.tritium

beta_tritium

beta other

sample information

beta_other

beta_other

beta_other

beta_other

beta_other

beta_other

beta other

beta_other

beta_other

beta_other

gamma_screen

- sample_tracking_id

- customer_sample_name

detector_count_length

retector_count_length_-

results_needed by t $t$ ime

result report citation

any_special instructions

\section{.sample_tracking_id}

. cust omer_sample_name

. detector_count_length

need_beta_nickel 1 a_iron_55

. need_beta_iron_55

need beta plutonium 241

results needed by date

results_needed by time

date_all_work_completed .any_special_instructions

rmation

. sample_tracking_id

detector count length

detector count length units

results_needed_by_date
1 ident if ication_codes

2 sixty_characters

20 real_numbers

21 units

22 integer_date_count

23 character_time_hh_mm

25 twenty characters

26 flags

$\mathrm{f} / \mathrm{p} \quad \mathrm{b} \quad 1$ identification_codes 2 sixty_characters

28 real_numbers

29 units

30 integer_date_count

31 character $t$ ime_hh mm

33 twenty_characters

34 flags

1 identification_codes 2 sixty_characters

36 real_numbers

37 units
38 flags

38 flags
39 flags

40 flags

41 flags

42 integer_date_count

43 character_time hh $\mathrm{mm}$

44 integer_date_count

45 ewenty_character

46 Elags

$f / p \quad g \quad 1$ identification_codes 2 sixty_character

5 real num

6 integer_date_count 
gamma_screen

gamma_screen

(a)

gamia screen

gamma screen

gamma_full_isotopic

sample_information

gamma_ful1

gamma_full_isotopic

gamma_tul1_isotopic

gamma_full_isotopic

gamma_ful1_isotopic

gamma_full-isotopic

gamma full isotopic

gamma_ful1_isotopic .results_needed_by_time date_al__work_completed date_sample_counted is this al ict results report citation any special instruct ions

sample_tracking_id

customer_sample_name

detector_count_length

results_needed_by_date

results needed_by_time

date sample counted

rmi_spectral 1 id

is_this_a spectrum recount

results_report_citation
7 character_time_hb_mm integer_date_count

10 integer date count

codes

13 twenty_characters

1 identification_codes

2 sixty_characters

15 real_numbers

16 units

17 integer_date oxunt

18 character_t ist hh_m

19 integer_date ... ount

21 integer datal id codes

22 flags.

23 twenty_characters gamma_full isotopic

.any_special_instructions

gamma_other

gamma_other

gamma_other

gamma_other

gamma_other

gamma_other

gamma ot her

gamma other

gamma_other

gross_alpha_beta_air_filters

sample information

gross_alpha_beta_air_filters

gross_alpha_beta_air_filter

gross alpha

gross alpha beta air filters

gross alpha beta air filters

gross_alpha_beta_air_filters

gross_alpha_beta_other

sample_information

gross_alpha_beta_other

grossalpha_beta_othe

grossalpha -beta_other

grose alpha bet other

gross alpha beta other

gross_alpha_beta_other

sample_information

special inst ruction

special instruction

- n-n-_-n

results_needed_by_time sample_tracking_id

detector_count_length

detector_count_length_units

results_needed_by_date

date_al _ work_conpleted

rml spect ral id

is_this_a_spectrum_recount results_report_citation

sample_tracking_id

customer_sample_name

detector_count_length

_ength_unit

.

date all work complete results report citation any_special_instructions

\section{sample_tracking_id}

customer_sample_name

detector_count_length

detector_count_length_unit

results needed by time

date ali work completed resuits report citation any_special_inst ructions

sample_tracking_id

.

origin of special_instruction

促 $\mathrm{f} / \mathrm{p}$

24 flags

1 identification_codes

2 sixty_characters

26 real_n

28 integer_date_count

29. character_time_hh_m

30 integer_date_count

12 integer date count

32 rmlaspect

34 twenty_characters

$\mathrm{f} / \mathrm{p} \mathrm{c} \quad 1$ ident if ication_codes

2 sixty_characters

4 real_number

6 integer_date_count

integer date count

twenty_characters

10 flags

$f / p \quad c \quad 1$ identification_codes

2 sixty_character

12 real_number

14 units

15 integer_date_count

16 integer date_count

17 twenty_characters

18 flags
1 ident if ication_codes
2 sixty_characters
3 sixteen_characters
nd integer nuacters 
tracking

sample_information

tracking

tracking

tracking

racking

tracking

tracking sample_tracking_id

customer_sample_name

possessor_name

ession reason

dat relinguished possessor

possessor_sequence_number
$f / p 1 t \quad \frac{1}{2}$ identification_codes 2 sixty_characters
3 names 4 phone_numbers
5 sixty_characters
fifty_characters
integer_date_count
nd integer numbers




\section{B.2. Screen-Sorted List}

Worksheet for Sorting Entities and Attributes and Assigning Keys and Relationships.

Base Worksheet - Sorted by Screen

\begin{tabular}{|c|}
\hline $\begin{array}{l}\text { Entity } \\
\text { sample_informat ion } \\
\text { sample_informat ion } \\
\text { sample_informat ion } \\
\text { sample_informat ion } \\
\text { sample_informat ion } \\
\text { sample_informat ion } \\
\text { sample_informat ion } \\
\text { sample_informat ion } \\
\text { sample_informat ion } \\
\text { sample_informat ion } \\
\text { sample_informat ion } \\
\text { sample_informat ion } \\
\text { sample_informat ion } \\
\text { sample_informat ion } \\
\text { sample_informat ion } \\
\text { sample_information } \\
\text { sample_informat ion } \\
\text { sample_informat ion } \\
\text { sample_informat ion } \\
\text { sample_informat ion } \\
\text { sample_informat ion } \\
\text { sample_informat ion } \\
\text { sample_informat ion } \\
\text { sample_informat ion } \\
\text { sample_informat ion } \\
\text { sample_informat ion } \\
\text { sample_informat ion } \\
\text { sample_informat ion } \\
\text { tracking } \\
\text { tracking } \\
\text { tracking } \\
\text { sample_informat ion } \\
\text { sample_informat ion } \\
\text { sample_informat ion } \\
\text { sample_informat ion } \\
\text { sample_informat ion } \\
\text { sample_informat ion } \\
\text { sample_informat ion }\end{array}$ \\
\hline
\end{tabular}

Attribute

- sample tracking id

- date_sample_entered

. date_all_work_completed

. customer_sample id

-results_needed_by_date

-customer_project_name

-work_charge_number

. customer_sample_name

- sample_type

. sample_size

. sample_size_units

- sample collection date

- sample collection time

. any special_instructions

- sample_hp_surveyed

- sample_hp_survey_activity

- sample_submitter_name

-sample_submitter_phone_number

- sample_submitter_address

- technical_contact_name

- technical_contact_phone_number

. technical_contact_addreșs

- send_results_to_name

- send_results_to_phone_number

-send_results_to_adaress

. sample_pickup_by name

- sample_pickup_by_phone_number

. sample_pickup_by_address

- possessor_name

- possessor_phone_number

- possessor_address

-chain_of_custody_number

-need_gross_alpha_beta

- need a lpha

- need_beta

- need-gamma

- next_possessor_sequence_number

- next_special_instruction_line

.sample_tracking_id

- customer sample name

- need a lpha uranium

-results_needed_by_date

.date_alī_work_completed

-results_report_citation

-any_special_instructions

- need alpha_thorium

-resuIts_needed_by_date

- date ali work completed

, results report citation

- results_report_citation

any_special inst ructio

-resuits needed_by_date

. date ali work completed

- results report citation

-any_special_instructions

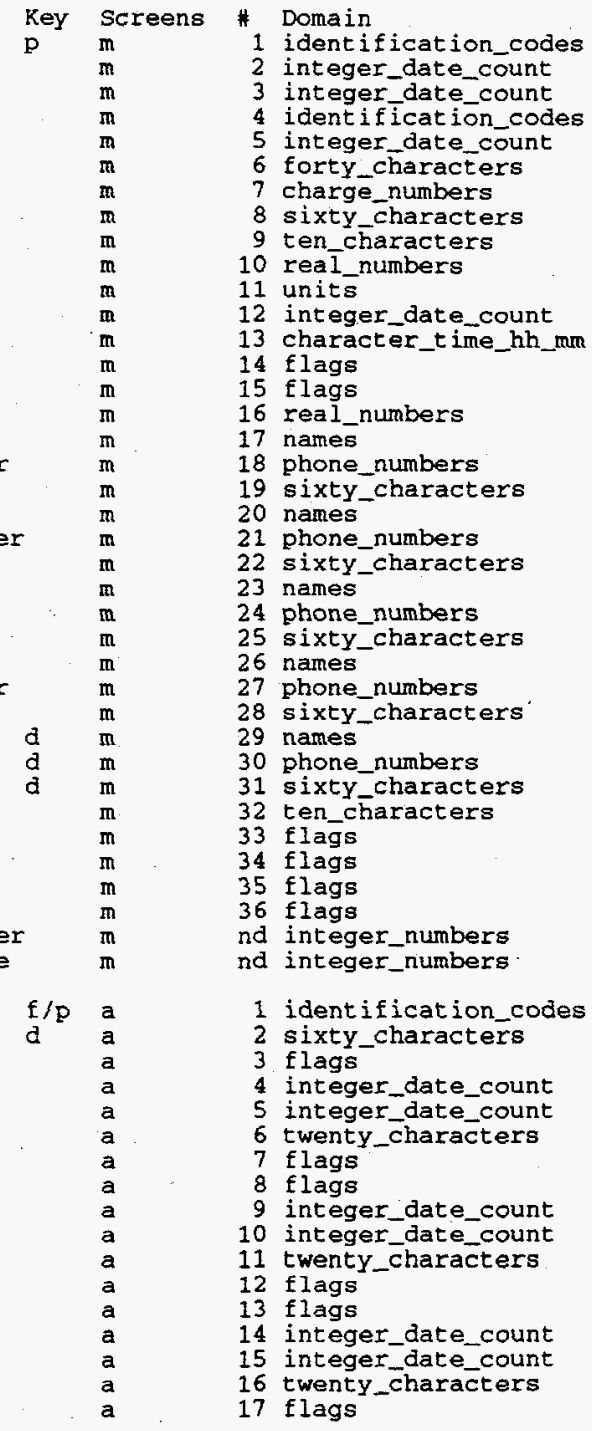

sample informat ion

alpha_am241_sans_pu238

alpha_am241_sans_pu238

alpha_am241_sans_pu238

alpha_am241_sans_pu238

sample information

alpha_am241_with_pu238

alpha am 241 with pu 238

alpha_am241_with_pu238

alpha_am241_with_pu238

sample_information

alpha_total_spectrometric

alpha_total_spectrometric

alpha_total_spectrometric need alpha am 241 sans pu238

- results_needed_by_date

-date_alín_work_completed

-resuīts_report_citation

-any_special_instructions

- need alpha am241_with pu238

- results needed by date

- date ali work completed

.results_report_citation

-results_report_citation

- need_alpha_total_spectrometric

-results_needed_by_date

.date_al1 work_completed

-results_report_citation
18 flags

19 integer_date_count

20 integer_date_count

21 twenty_characters

22 flags

23 flags

24 integer_date_count

25 integer date count

26 twenty_characters

27 flags

28 flags

29 integer_date_count

30 integer date count

31 twenty characters 
alpha_total_spectrometric sample information

alpha other

alpha_other

alpha_other

alpha_other

beta_strontium_90

sample_information

sample information

beta stront ium 90

beta_strontium_90

beta_strontium_90

beta_strontium_90

beta_strontium_90

beta strontium_90

beta_strontium 90

sample_information

beta_strontium_89_and_90

beta_strontium_89_and_90

beta strontium 89 and 90

beta_strontium_89_and_90

beta stront ium 89 and 90

beta strontium_89-and 90

beta_strontium_89_and_90

sample_information

beta_total_strontium

beta total strontium

beta_total_strontium

beta total strontium

beta_total_strontium

beta_total_strontium

beta_total_strontiun

sample_information

beta_tritium

beta_tritium

beta tritium

beta_tritium

beta-tritium

beta_tritium

beta_tritium

sample information

beta other

beta_other

beta-other

beta_other any_special_instructions

need alpha other

-results_needed_by_date

date_ali_work_completed

resuIts_report_citation

- any_special instructions

-sample_tracking_id

- customer sample_name

need betástrontium 90

- detector_count_length

- detector_count_length_units

results.ñeeded_by_date

results_needed_by_time

date_all_work_completed

results report_citation

- any_special_instructions

need_beta_strontiun_89_and_90

-detector_count_length

-detector_count_length_units

-results_needed_by_date

-results_needed_by_time

date ali work completed

results_report_citation

any_special_instructions

need_beta_total_stront ium

- detector_count_length

.detector_count_length_units

results_needed_by_date

- results needed by time

- date_ali_work_completed

results_report_citation

. any_special_instructions

- need_beta_tritium

- detector_count_length

detector count length units

results_needed_by_date

results needed_by_time

date_ali_work_completed

results_report_citation

- any special instructions

need beta other

.detector_count_length

- detector_count_length_units

- need_beta_nickel 63

- need_beta_iron_55
32 flags

33 flags

34 integer_date_count

35 integer_date_count

36 twenty_characters

37 flags

1 identification_codes

2 sixty_characters

3 flags

4 real_numbers

5 units

6 integer_date_count

7 character time_hh_mm

8 integer date count

9 twenty characters

10 flags

11 flags

12 real_numbers

13 units

14 integer_date_count

15 character_time_hh_mm

16 integer_date_count

17 twenty_characters

18 flags

19 flags

20 real_numbers

21 units

22 integer_date_count

23 character time hh mm

24 integer_date_count

25 twenty_characters

26 flags

27 flags

28 real numbers

29 units

30 integer date count

31 character_time_hh_mm

32 integer_date_count

33 twenty_characters

34 flags

35 flags

36 real numbers

37 units

38 flags

39 flags beta_other

beta_other

beta_other

beta other

beta_other

beta other

beta_other

gamma_screen

sample information

sample information

gamma screen

gamma screen

gamma_screen

gamma screen

gamma_screen

gamma screen

gamna screen

gamma_screen

gamma_screen

gamma_screen

sample_information

gamma_full_isotopic

gamma full isotopic

gamma full isotopic

gamma full-isotopic

gamma full isotopic

gamma_full_isotopic

gamna_ful1_isotopic

gamma_full_isotopic

gamma_full_isotopic
- need_beta_sulfur_35

need_beta_plutonium_241

-results_needed_by_dāte

results needed by time

- date ali work completed

-results_report_citation

any_special_instructions

sample_tracking_id

cust omer sample name

need gamma screen

. detector_count_length

detector_count_length_units

results_ñeeded_by_date

results needed_by_time

date_ali_work_completed

date_sample_counted

rml spectral id

is_this_a spectrum recount

- results_report citation

any special instructions

need gamma full isotopic

detector_count_ilength

. detector_count_length_units

results_needed by date

results needed by $t$ ime

date alī work completed

date_sample_counted

rml_spectral_id

is_this_a_spectrum_recount

results report citation
40 flags

41 flags

42 integer_date_count

43 character $t$ ime hh mm

44 integer date count

45 twenty characters

46 flags

1 identification_codes

2 sixty_characters

3 flags

4 real_numbers

5 units

6 integer_date_count

7 character_time_hh_mm

8 integer_date_count

9 integer_date count

$10 \mathrm{rml}$ spectral id codes

11 flags

12 twenty characters

13 flags

14 flags

15 real_numbers

16 units

17 integer_date_count

18 character time hh

19 integer date count

19 integer_date_count

20 integer_date_count

$21 \mathrm{rml}$ spectral_id_codes

22 flags

23 twenty_characters gamma_full_isotopic sample_information any special instructions

need gamma other
24 flags
25 flags 
gamma_other

gamina_other

gamma_other

gamma_other

gamma_other

gamma_othe

ganma_other

gamma_other

amma_other

gamma_other

gross_alpha_beta_air_filters sample_.informat ion

sample_information

gross_alpha_beta_air filters

grass_alpha_beta_air_filters gross_alpha_beta_air_filters gross_alpha_beta_air_filters gross_alpha_beta_air_filters gross_alpha_beta_air_filters gross_alpha beta air filters sample in formation

gross alpha beta other gross_alpha_beta_other gross_alpha_beta_other gross_alpha_beta_other gross_alpha_beta_other gross_alpha_beta_other -detector_count_length

detector_count length_units

.results_needed_by_date

results needed by time

- date_ali__work_completed

. date_sample_counted

- rmi_spectral_id

- is_this_a_spectrum_recount

results report citation

any_special_inst ructions

sample_tracking_id

customer_sample_name

need_gross_alpha_beta_filter

detector count length

detector count length units

. results_needed_by_date

. results_needed_by_time

.date_alī_work_completed

-results_report_citation

- any_special_instructions

- need gross_alpha beta other

. detector_count_length

- detector count length units

results_needed_by_date

results_needed_by_time

date_al $\bar{l}_{\text {_work_completed }}$

results report citation.
26 real_numbers

27 units

28 integer date count

29 character_time_hh_mm

30 integer_date_count

31 integer_date_count

32 rml_spectral_id_codes

33 flags

34 twenty_characters

35 flags

1 identification_codes

2 sixty_characters

3 flags

4 real_numbers

5 units

6 integer_date_count

7 character_time_hh mm

8 integer_date_count

9 twenty_characters

10 flags

12 real numbers

13 units

14 integer_date_count

15 character_time_hh_mm

16 integer_date_count

17 twenty_characters gross_alpha_beta_other

special_instructions sample_information

special_instructions

special instructions

special_instructions

tracking

sample information

tracking

tracking

tracking

tracking

tracking

tracking

tracking .any_special_instructions

. sample_tracking_id

customer_sample_name

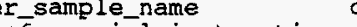

special_instruction

-special_instruction_line

p3

sample tracking id

customer sample name

possessor_name

possessor_phone_number

- possessor_address

- possessor_possession_reason

-date_accepted_by_possessor

date_relinguished by possessor

-possessor_sequence_number c

18 flags

1 ident if ication codes

2 sixty_characters

sixteen_characters

4 fifty_characters

nd integer_numbers

1 identification_codes

2 sixty_characters

3 names

4 phone_numbers

5 sixty_characters

6 fifty_characters

7 integer_date_count

integer date count

nd integer_numbers 


\section{EXECUTION OF CREATE SCHEMA - A SAMPLE TERMINAL SESSION}

What the user enters appears in bold text in the following.

\section{\$ Ecreate_schema}

Enter the name of the schema to be created: sample_tracking

For domain CHARACTER_TIME_HH_MM:

Enter the data type for this domain (char/date/int/real): c

Enter the length of the character domain: 5

Enter the default value (return says NULL):

Enter any desired comment: Domain for time in the form hh:mm.

For domain CHARGE_NUMBERS:

Enter the data type for this domain (char/date/int/real): c

Enter the length of the character domain: 9

Enter the default value (return says NULL): PF7400000

Enter any desired comment: Domain for nine-alphanumeric-long charge numbers.

For domain FIFTY_CHARACTERS:

Enter the data type for this domain (char/date/int/real): c

Enter the length of the character domain: $\mathbf{5 0}$

Enter the default value (return says NULL):

Enter any desired comment: Domain for fifty-alphanumeric-long fields. For domain FLAGS:

Enter the data type for this domain (char/date/int/real): c

Enter the length of the character domain: 1

Enter the default value (return says NULL): $\mathbf{N}$ flags.

Enter any desired comment: Domain for one-character-long yes/no

For domain FORTY_CHARACTERS:

Enter the data type for this domain (char/date/int/real): c

Enter the length of the character domain: $\mathbf{4 0}$

Enter the default value (return says NULL): 
Enter any desired comment: Domain for forty-alphanumeric-long fields. For domain IDENTIFICATION_CODES:

Enter the data type for this domain (char/date/int/real): c Enter the length of the character domain: 12

Enter the default value (return says NULL):

Enter any desired comment: Dowain for twelve-character-long identification codes.

For domain INTEGER_DATE_COUNT:

Enter the data type for this domain (char/date/int/real): 1

Enter the default $\therefore$ ie (return says NULL):

Enter any desired comment: Domain for integer*4 date count starting at January 1, 1990.

For domain INTEGER_NUMBERS:

Enter the data type for this domain (char/date/int/real): 1

Enter the default value (return says NULL):

Enter any desired comment: Domain for integer*4 numbers.

For domain NAMES:

Enter the data type for this domain (char/date/int/real): c

Enter the length of the character domain: 25

Enter the default value (return says NULL):

Enter any desired comment: Domain for twenty-five-alphanumeric-1ong names.

For domain PHONE_NUMBERS:

Enter the data type for this domain (char/date/int/real): c

Enter the length of the character domain: 12

Enter the default value (return says NULL):

Enter any desired comment: Domain for twelve-alphanumeric-long phone numbers.

For domain REAL_NUMBERS:

Enter the data type for this domain (char/date/int/real): $x$

Enter the default value (return says NULL):

Enter any desired comment: Domain for real*4 numbers. 
For domain RML_SPECTRAL_ID_CODES:

Enter the data type for this domain (char/date/int/real): c

Enter the length of the character domain: 14

Enter the default value (return says NULL):

Enter any desired comment: Domain for fourteen-character-long RML spectral identification codes (<save area>\$<spectral id>).

For domain SIXTEEN_CHARACTERS:

Enter the data type for this domain (char/date/int/real): c

Enter the length of the character domain: 16

Enter the default value (return says NULL): fields.

Enter any desired comment: Domain for sixteen-alphanumeric-1ong

For domain SIXTY_CHARACTERS:

Enter the data type for this domain (char/date/int/real): c Enter the length of the character domain: 60

Enter the default value (return says NULL):

Enter any desired comment: Domain for sixty-alphanumeric-long fields.

For domain TEN_CHARACTERS:

Enter the data type for this domain (char/date/int/real): c

Enter the length of the character domain: 10

Enter the default value (return says NULL):

Enter any desired comment: Domain for ten-alphanumeric-long fields. For domain TWENTY_CHARACTERS:

Enter the data type for this domain (char/date/int/real): c

Enter the length of the character domain: $\mathbf{2 0}$

Enter the default value (return says NULL): fields.

Enter any desired comment: Domain for twenty-alphanumeric-long

For domain UNITS:

Enter the data type for this domain (char/date/int/real): c Enter the length of the character domain: 5 
Enter the default value (return says NULL):

Enter any desired comment: Domain for five-alphanumeric-1ong unita. For table SAMPLE_INFORMATION:

Enter any desired comment: Table for sample information.

Enter the number of rows per page to be clustered together: 1

Enter the maximum number of rows for this table: $\mathbf{4 0 0 0}$

For table ALPHA_URANIUM:

Enter any desired comment: Table for requested alphe analyses for Uranium radionuclides.

For foreign key SAMPLE_TRACKING_ID:

Enter the reference table: SAMPLE_IMFORMATION

Enter the reference column: SAMPLE_TRACKING_ID

Enter the number of rows per page to be clustered together: 1

Enter the maximum number of rows for this table: 1000

For table ALPHA_THORIUM:

Enter any desired comment: Table for requested alpha analyses for Thorium radionuclides.

For foreign key SAMPLE_TRACKING_ID:

Enter the reference table: SAMPLE_IMFORMATION

Enter the reference column: SAMPLE_TRACKING_ID

Enter the number of rows per page to be clustered together: 1

Enter the maximum number of rows for this table: 1000

For table ALPHA_PLUTONIUM:

Enter any desired comment: Table for requested alpha analyses for Plutonium radionuclides.

For foreign key SAMPLE_TRACKING_ID:

Enter the reference table: SAMPLB_INFORMaTION

Enter the reference column: SAMPLB_TRACKING_ID

Enter the number of rows per page to be clustered together: 1

Enter the maximum number of rows for this table: 1000

For table ALPHA_AM241_SANS_PU238:

Enter any desired comment: Table for requested alpha analyses for 
Americium-241 separate from Plutonium-238.

For foreign key SAMPLE_TRACKING_ID:

Enter the reference table: SAMPLE_INFORMATION

Enter the reference column: SAMPLE_TRACKING_ID

Enter the number of rows per page to be clustered together: 1

Enter the maximum number of rows for this table: 1000

For table ALPHA_AM241_WITH_PU238:

Enter any desired comment: Table for requested alpha analyses for Americium-241 combined with Plutonium-238.

For foreign key SAMPLE_TRACKING_ID:

Enter the reference table: SAMPLE_INFORMATION

Enter the reference column: SAMPLE_TRACKING_ID

Enter the number of rows per page to be clustered together: 1

Enter the maximum number of rows for this table: 1000

For table ALPHA_TOTAL_SPECTROMETRIC:

Enter any desired comment: Table for requested total spectrometric alpha analyses.

For foreign key SAMPLE_TRACKING_ID:

Enter the reference table: SAMPLB_IMFORMATION

Enter the reference column: SAMPLE_TRACKING_ID

Enter the number of rows per page to be clustered together: 1

Enter the maximum number of rows for this table: 1000

For table ALPHA_OTHER:

Enter any desired comment: Table for requested alpha analyses for other, unlisted radionuclides.

For foreign key SAMPLE_TRACKING_ID:

Enter the reference table: SAMPLE_IMFORMATION

Enter the reference column: SAMPLE_TRACKING_ID

Enter the number of rows per page to be clustered together: 1

Enter the maximum number of rows for this table: 1000

For table BETA_STRONTIUM_90:

Enter any desired comment: Table for requested beta analyses for 
Strontium-90.

For foreign key SAMPLE_TRACKING_ID:

Enter the reference table: SAMPLE_INFORMATION

Enter the reference column: SAMPLE_TRACKIMG_ID

Enter the number of rows per page to be clustered together: 1

Enter the maximum number of rows for this table: 1000

For table BETA_STRONTIUM_89_AND_90:

Enter any desired comment: Table for requested beta analyses for strontium-89 and -90 .

For foreign key SAMPLE_TRACKING_ID:

Enter the reference table: SAMPLE_INFORMATION

Enter the reference column: SAMPLB_TRACKING_ID

Enter the number of rows per page to be clustered together: 1

Enter the maximum number of rows for this table: 1000

For table BETA_TOTAL_STRONTIUM:

Enter any desired comment: Table for requested beta analyses for total strontium.

For foreign key SAMPLE_TRACKING_ID:

Enter the reference table: SAMPLE_INFORMaTION

Enter the reference column: SAMPLE_TRACKING_ID

Enter the number of rows per page to be clustered together: 1

Enter the maximum number of rows for this table: 1000

For table BETA_TRITIUM: Tritium.

Enter any desired comment: Table for requested beta analyses for

For foreign key SAMPLE_TRACKING_ID:

Enter the reference table: SAMPLE_INFORMaTION

Enter the reference column: SAMPLE_TRACKING_ID

Enter the number of rows per page to be clustered together: 1

Enter the maximum number of rows for this table: 1000

For table BETA_OTHER:

Enter any desired comment: Table for requested beta analyses for 
other, unlisted radionuclides.

For foreign key SAMPLE_TRACKING_ID:

Enter the reference table: SAMPLE_IMFORMATION

Enter the reference column: SAMPLB_TRACKING_ID

Enter the number of rows per page to be clustered together: 1

Enter the maximum number of rows for this table: 1000

For table GAMMA_SCREEN:

Enter any desired comment: Table for requested screening and shipping gamma analyses.

For foreign key SAMPLE_TRACKING_ID:

Enter the reference table: SAMPLE_INPORMATION

Enter the reference column: SAMPLE_TRACKING_ID

Enter the number of rows per page to be clustered together: 1

Enter the maximum number of rows for this table: $\mathbf{4 0 0 0}$

For table GAMMA_FULL_ISOTOPIC:

Enter any desired comment: Table for requested full 1sotopic gamma analyses.

For foreign key SAMPLE_TRACKING_ID:

Enter the reference table: SAMPLE_INFORMATION

Enter the reference column: SAMPLE_TRACKING_ID

Enter the number of rows per page to be clustered together: 1

Enter the maximum number of rows for this table: $\mathbf{4 0 0 0}$

For table GAMMA_OTHER:

Enter any desired comment: Table for requested gamma analyses for other, unlisted radionuclides.

For foreign key SAMPLE_TRACKING_ID:

Enter the reference table: SAMPLB_INFORMATION

Enter the reference column: SAMPLE_TRACKING_ID

Enter the number of rows per page to be clustered together: 1

Enter the maximum number of rows for this table: $\mathbf{4 0 0 0}$

For table GROSS_ALPHA_BETA_AIR_FILTERS:

Enter any desired comment: Table for requested gross alpha-beta 
analyses of air filters.

For foreign key SAMPLE_TRACKING_ID:

Enter the reference table: SAMPLE_INFORMatION

Enter the reference column: SAMPLE_TRACKING_ID

Enter the number of rows per page to be clustered together: 1

Enter the maximum number of rows for this table: 1000

For table GROSS_ALPHA_BETA_OTHER:

Enter any desired comment: Table for requested grose alpha-beta analyses of other, unlisted samples.

For foreign key SAMPLE_TRACKING_ID:

Enter the reference table: SAMPLE_INFORMATION

Enter the reference column: SAMPLE_TRACKING_ID

Enter the number of rows per page to be clustered together: 1

Enter the maximum number of rows for this table: 1000

For table SPECIAL_INSTRUCTIONS:

Enter any desired comment: Table for special instructions.

For foreign key SAMPLE_TRACKING_ID:

Enter the reference table: SAMPLE_INFORMaTION

Enter the reference column: SAMPLE_TRACKIMG_ID

Enter the number of rows per page to be clustered together: 15

Enter the maximum number of rows for this table: 20000

For table TRACKING:

Enter any desired comment: Table for tracking samples.

For foreign key SAMPLE_TRACKING_ID:

Enter the reference table: SAMPLE_INPORMarIor

Enter the reference column: SAMPLB_TRACKING_ID

Enter the number of rows per page to be clustered together: 5

Enter the maximum number of rows for this table: 20000

Execution has successfully completed. The create_schema.sql and cursor and insert files will be closed and retained. The entity and screen files will also be closed and retained.

Please wait while certain sections of the cursor and insert files are 
rearranged; this may take a few minutes.

Cursor file rearranged.

Insert file rearranged.

$\$$ 


\section{SQL INSTRUCTION SET FOR CREATION OF SAMPLE TRACKING DATABASE}

Note the ellipses in the two "define/system" lines below; these would be replaced with the appropriate root directory specifier (\$USER:[DATABASES.], for example).

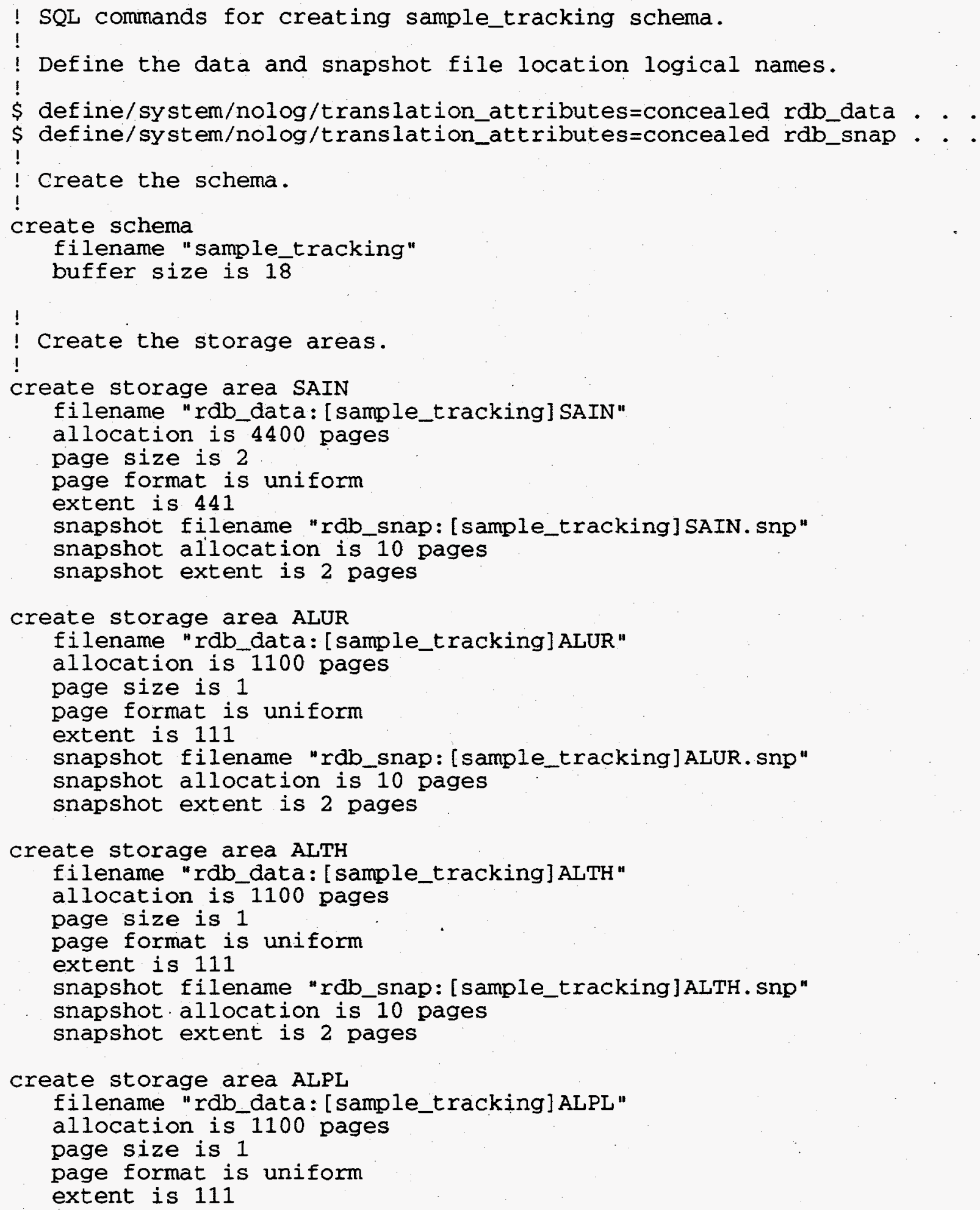


snapshot filename "rdb_snap:[sample_tracking]ALPL.snp"

snapshot allocation is 10 pages

snapshot extent is 2 pages

create storage area ALAMSAPU

filename "rdb_data: [sample_tracking] ALAMSAPU"

allocation is 1100 pages

page size is 1

page format is uniform

extent is 111

snapshot filename "rdb_snap: [sample_tracking]ALAMSAPU.snp"

snapshot allocation is 10 pages

snapshot extent is 2 pages

create storage area ALAMWIPU

filename "rdb_data: [sample_tracking] ALAMWI PU"

allocation is 1100 pages

page size is 1

page format is uniform

extent is 111

snapshot filename "rdb_snap:[sample_tracking]ALAMWIPU.snp"

snapshot allocation is 10 pages

snapshot extent is 2 pages

create storage area ALTOSP

filename "rdb_data: [sample_tracking] ALTOSP"

allocation is 1100 pages

page size is 1

page format is uniform

extent is 111

snapshot filename "rdb_snap:[sample_tracking] ALTOSP.snp"

snapshot allocation is 10 pages

snapshot extent is 2 pages

create storage area ALOT

filename "rdb_data: [sample_tracking] ALOT"

allocation is 1100 pages

page size is 1

page format is uniform

extent is 111

snapshot filename "rdb_snap:[sample_tracking]ALOT.snp"

snapshot allocation is 10 pages

snapshot extent is 2 pages

create storage area BEST90

filename "rob_data: [ sample_tracking] BEST90"

allocation is 1100 pages

page size is 1

page format is uniform

extent is 111

snapshot filename "rdb_snap: [sample_tracking] BEST90.snp"

snapshot allocation is 10 pages

snapshot extent is 2 pages

create storage area BEST89AN90

filename "rdb_data: [ sample_tracking] BEE. 39AN90"

allocation is 1100 pages

page size is 1

page format is uniform

extent is 111 
snapshot filename "rdb_snap:[sample_tracking] BEST89AN90.snp" snapshot allocation is 10 pages

snapshot extent is 2 pages

create storage area BETOST

filename "rdb_data: [sample_tracking] BETOST"

allocation is 1100 pages

page size is 1

page format is uniform

extent is 111

snapshot filename "rdb_snap:[sample_tracking]BETOST.snp"

snapshot allocation. is 10 pages

snapshot extent is 2 pages

create storage area BETR

filename "rdb_data: [sample_tracking] BETR"

allocation is 1100 pages

page size is 1

page format is uniform

extent is 111

snapshot filename "rdb_snap:[sample_tracking]BETR.snp"

snapshot allocation is 10 pages

snapshot extent is 2 pages

create storage area BEOT

filename "rdb_data: [sample_tracking] BEOT"

allocation is 1100 pages

page size is 1

page format is uniform

extent is 111

snapshot filename "rdb_snap:[sample_tracking]BEOT.snp"

snapshot allocation is 10 pages

snapshot extent is 2 pages

create storage area GASC

filename "rdb_data: [sample_tracking]GASC"

allocation is 4400 pages

page size is 1

page format is uniform

extent is 441

snapshot filename "rab_snap:[sample_tracking]GASC.snp"

snapshot allocation is 10 pages

snapshot extent is 2 pages

create storage area GAFUIS

filename "rdb_data: [sample_tracking] GAFUIS"

allocation is 4400 pages

page size is 1

page format is uniform

extent is 441

snapshot filename "rdb_snap: [sample_tracking]GAFUIS.snp"

snapshot allocation is 10 pages

snapshot extent is 2 pages

create storage area GAOT

filename "rdb_data: [ sample_tracking] GAOT"

allocation is 4400 pages

page size is 1

page format is uniform

extent is 441 


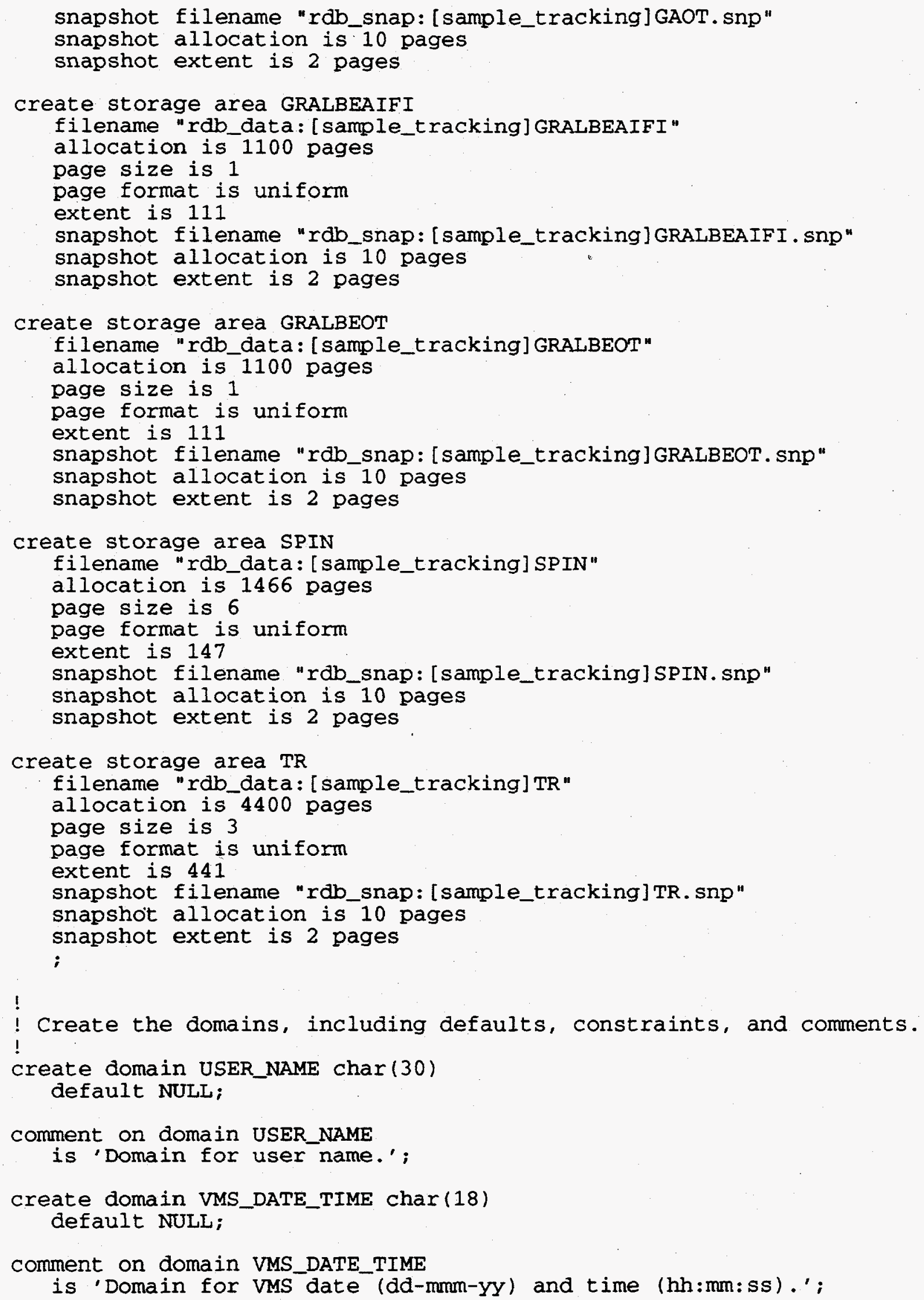




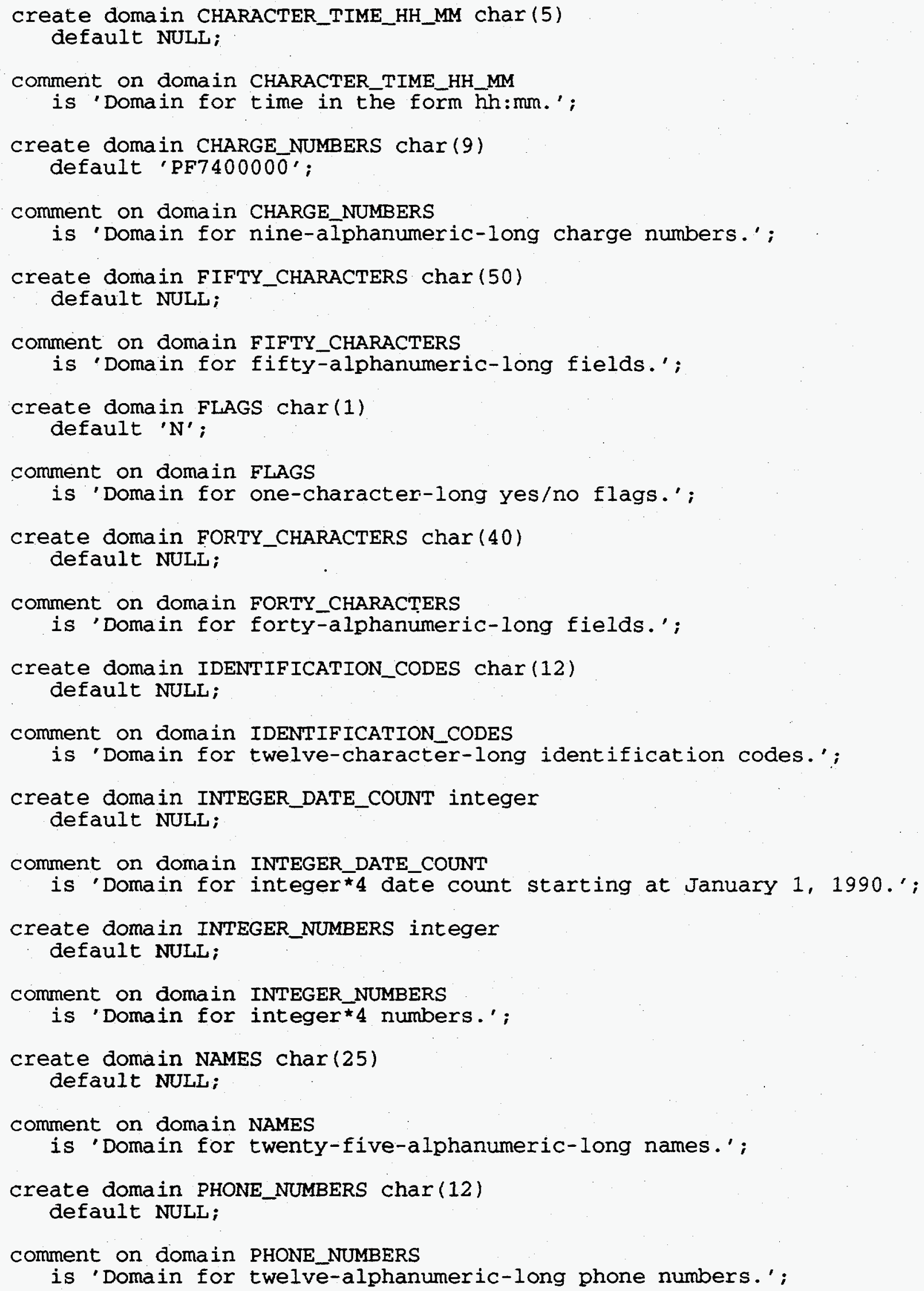




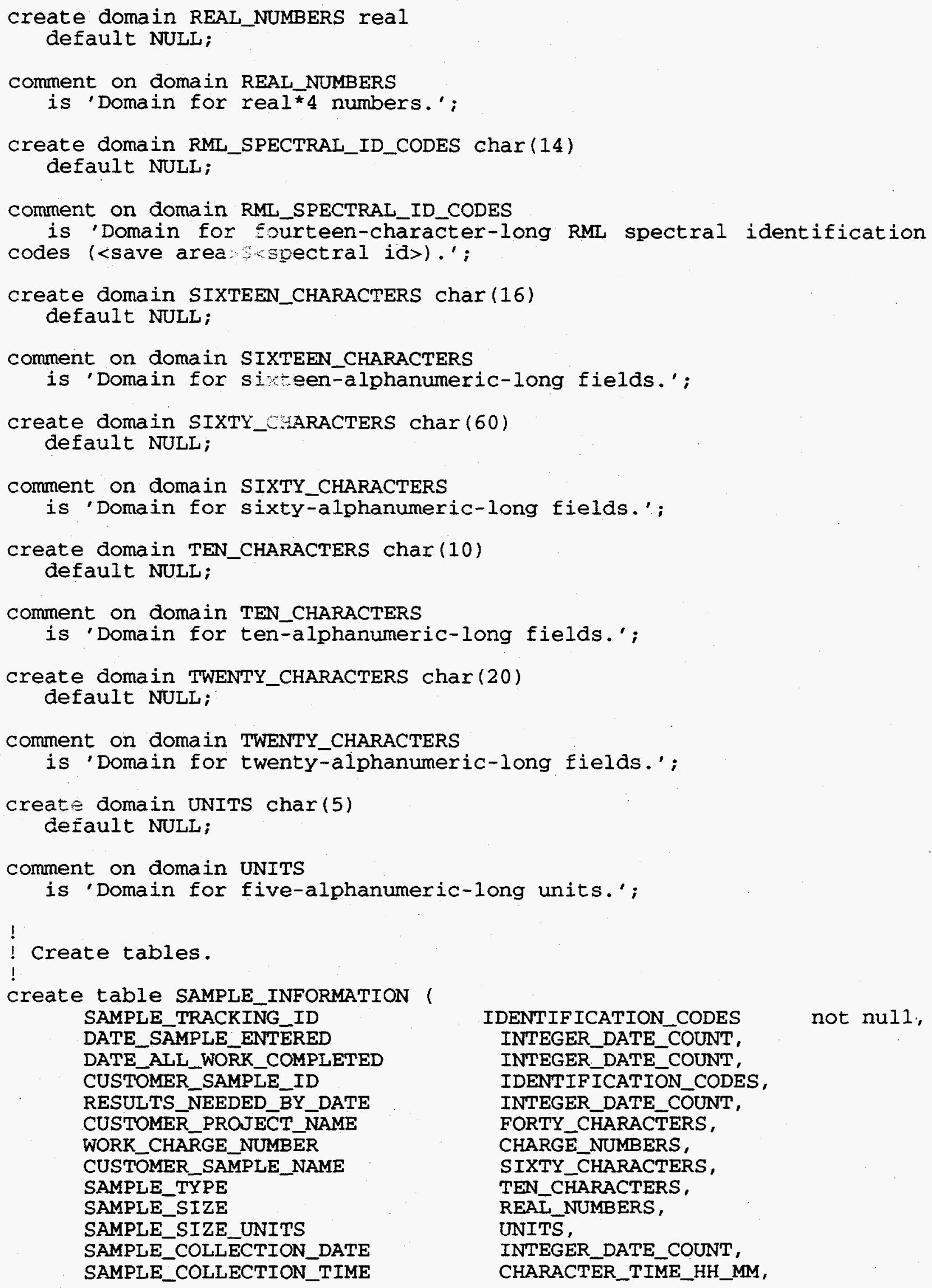




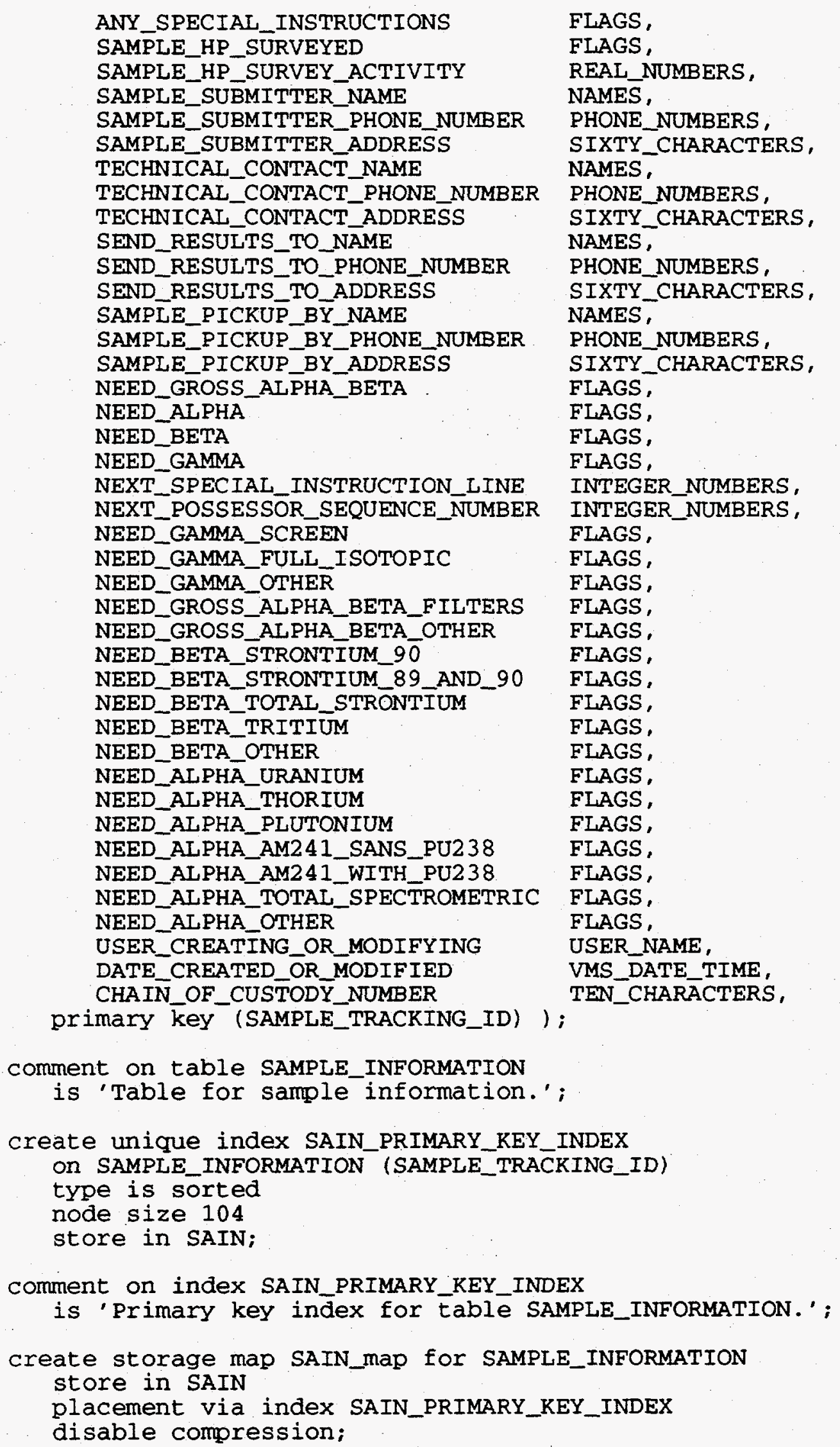




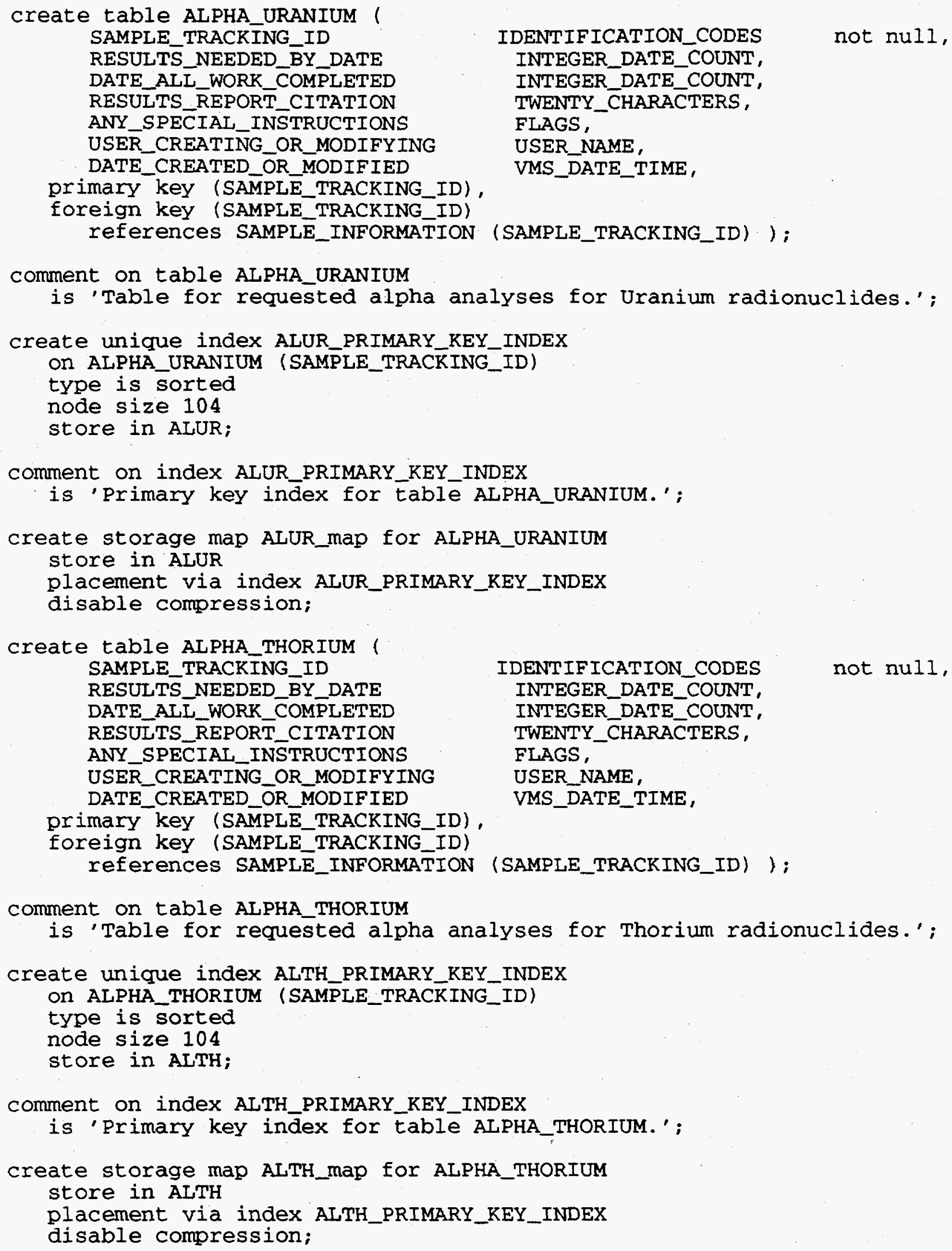




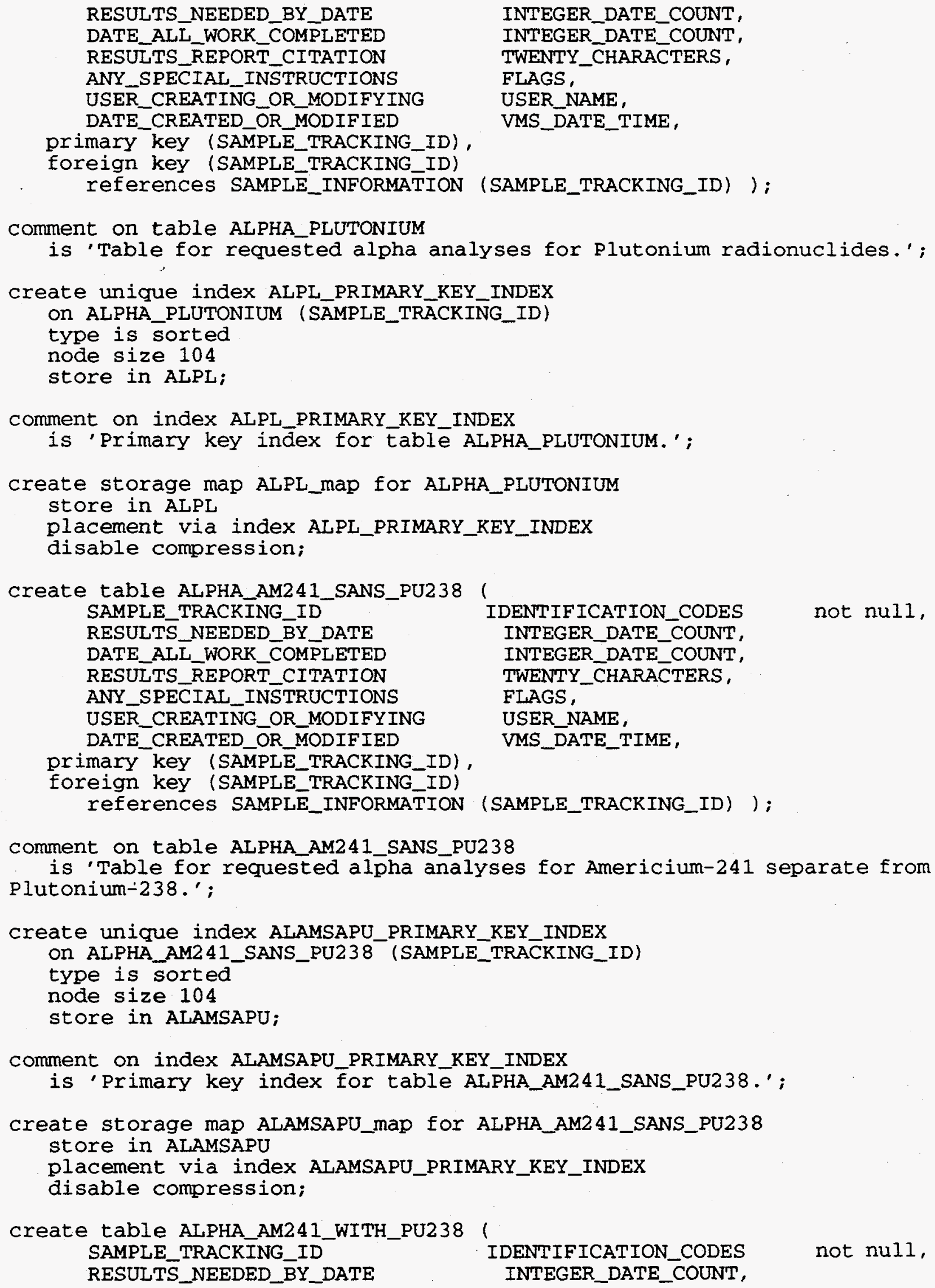


DATE ALL WORK COMPLETED RESULTS_REPORT_CITATION ANY_SPECIAL_INSTRUCTIONS USER_CREATING_OR_MODIFYING

DATE_CREATED_OR_MODIFIED

primary key (SAMPLE_TRACKING_ID)

foreign key (SAMPLE_TRACKING_ID)

references SAMPLE_INFORMATION (SAMPLE_TRACKING_ID) );
INTEGER_DATE COUNT, TWENTY_CHARACTERS,

FLAGS,

USER_NAME,

VMS DATE_TIME,

comment on table ALPHA_AM241_WITH_PU238

is 'Table for requested alpha analyses for Americium-241 combined with Plutonium-238.';

create unique index ALAMWIPU_PRIMARY_KEY_INDEX

on ALPHA_AM241_WITH_PU238 (SAMPLE_TRACKING_ID)

type is sorted

node size 104

store in ALAMWIPU;

comment on index ALAMWIPU_PRIMARY_KEY_INDEX

is 'Primary key index for table ALPHA_AM241_WITH_PU238.';

create storage map ALAMWIPU_map for ALPHA_AM241_WITH_PU238

store in ALAMWIPU

placement via index ALAMWIPU_PRIMARY_KEY_INDEX

disable compression;

create table ALPHA_TOTAL_SPECTROMETRIC

SAMPLE_TRACKING_ID

RESULTS_NEEDED_BY_DATE

DATE_ALE_WORK_COMPLETED

RESULTS_REPORT_CITATION

ANY_SPECIAL_INSTRUCTIONS

USER_CREATING_OR_MODIFYING

DATE_CREATED_OR_MODIFIED

IDENTIFICATION_CODES

INTEGER_DATE_COUNT,

not null, INTEGER_DATE_COUNT, TWENTY_CHARACTERS,

FLAGS, USER_NAME，

VMS_DATE_TIME,

primary key (SAMPLE_TRACKING_ID),

foreign key (SAMPLE_TRACKING_ID)

references SAMPLE_INFORMATION (SAMPLE_TRACKING_ID) );

comment on table ALPHA TOTAL_SPECTROMETRIC

is 'Table for requested total spectrometric alpha analyses.';

create unique index ALTOSP_PRIMARY_KEY_INDEX

On ALPHA_TOTAL_SPECTROMETRIC (SAMPLE_TRACKING_ID)

type is sorted

node size 104

store in ALTOSP;

comment on index ALTOSP_PRIMARY_KEY_INDEX

is 'Primary key index for table ALPHA_TOTAL_SPECTROMETRIC.';

create storage map ALTOSP_map for ALPHA_TOTAL_SPECTROMETRIC

store in ALTOSP

placement via index ALTOSP_PRIMARY_KEY_INDEX

disable compression;

create table ALPHA_OTHER ( SAMPLE_TRACKING_ID RESULTS_NEEDED_BY_DATE

DATE_ALE_WORK_COMPLETED

IDENTIFICATION_CODES not null,
INTEGER_DATE_COUNT,
INTEGER_DATE_COUNT,




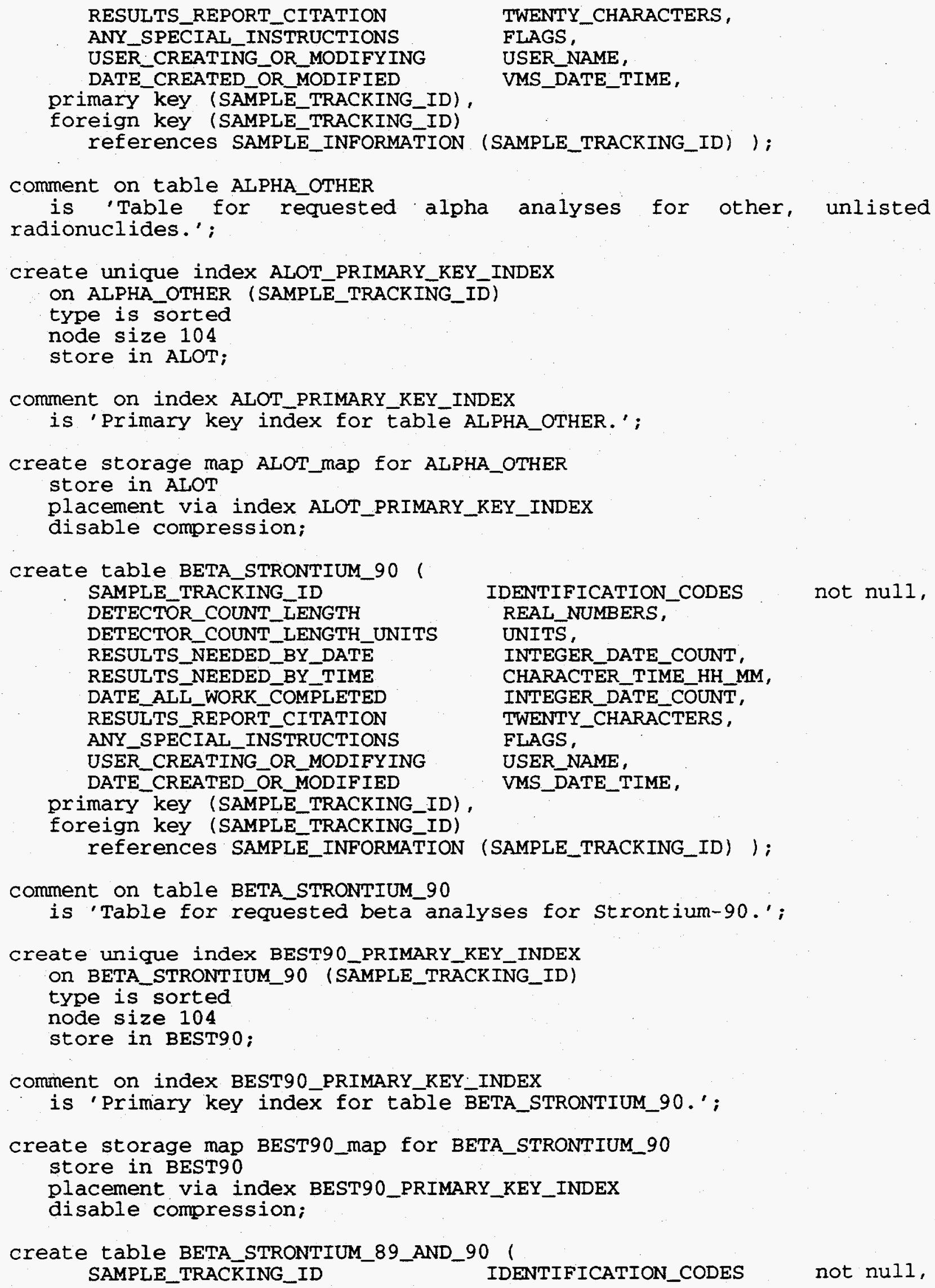$$
\text { REAL_NUMBERS, }
$$$$
\text { UNITS, }
$$ 


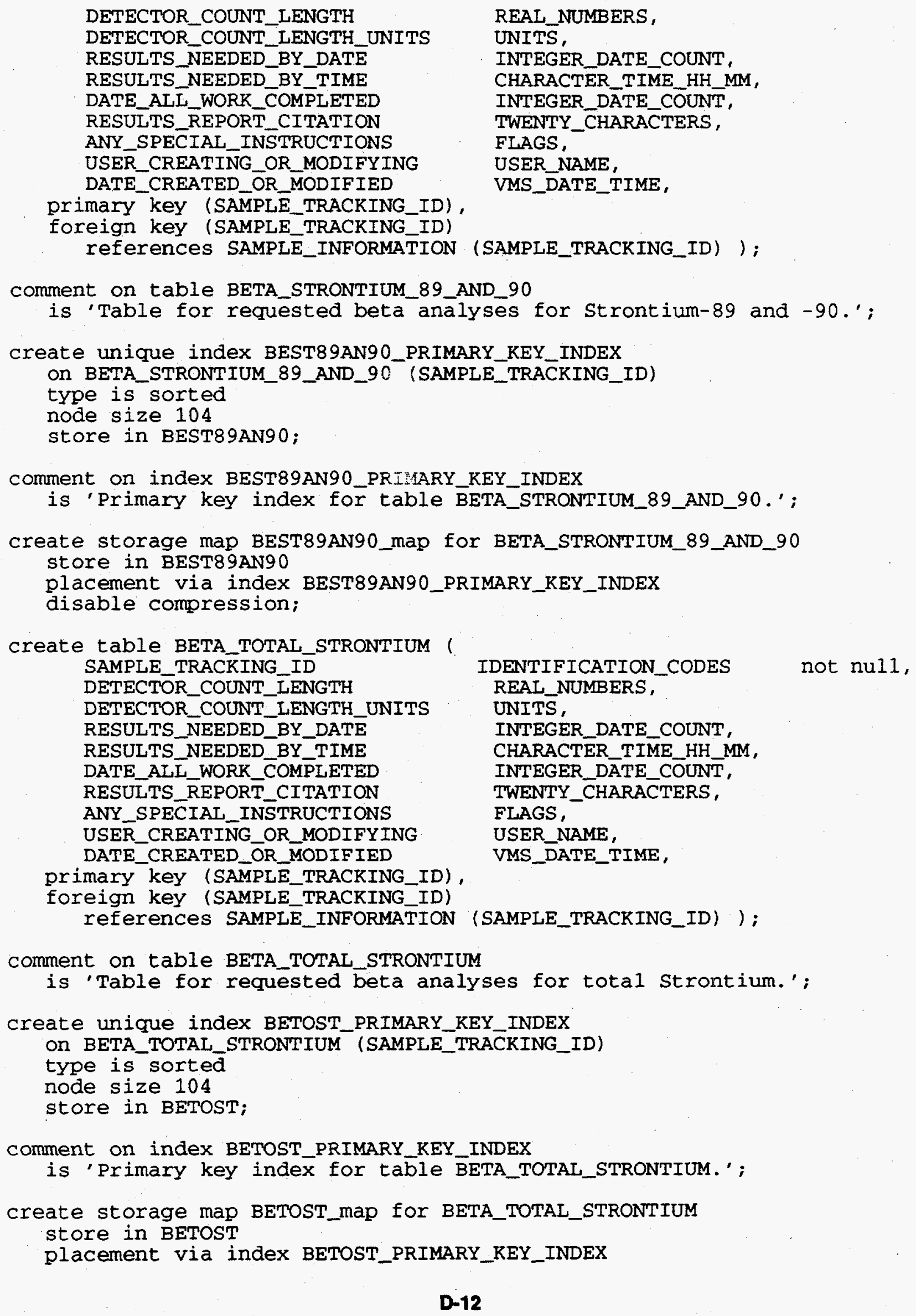


disable compression;

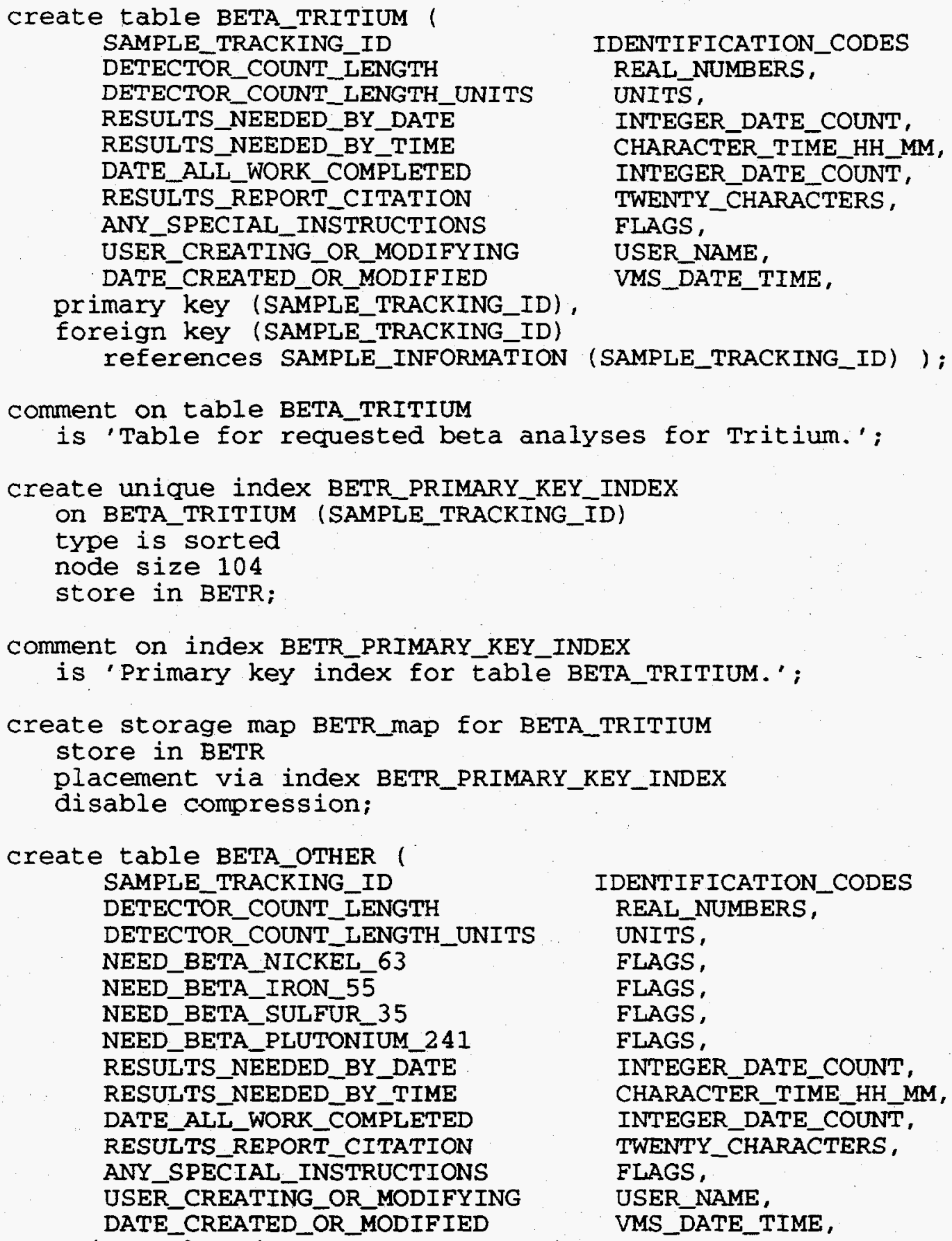


node size 104

store in BEOT;

comment on index BEOT_PRIMARY_KEY_INDEX

is 'Primary key index for table BETA_OTHER.';

create storage map BEOT_map for BETA_OTHER

store in BEOT

placement via index BEOT_PRIMARY_KEY_INDEX

disable compression;

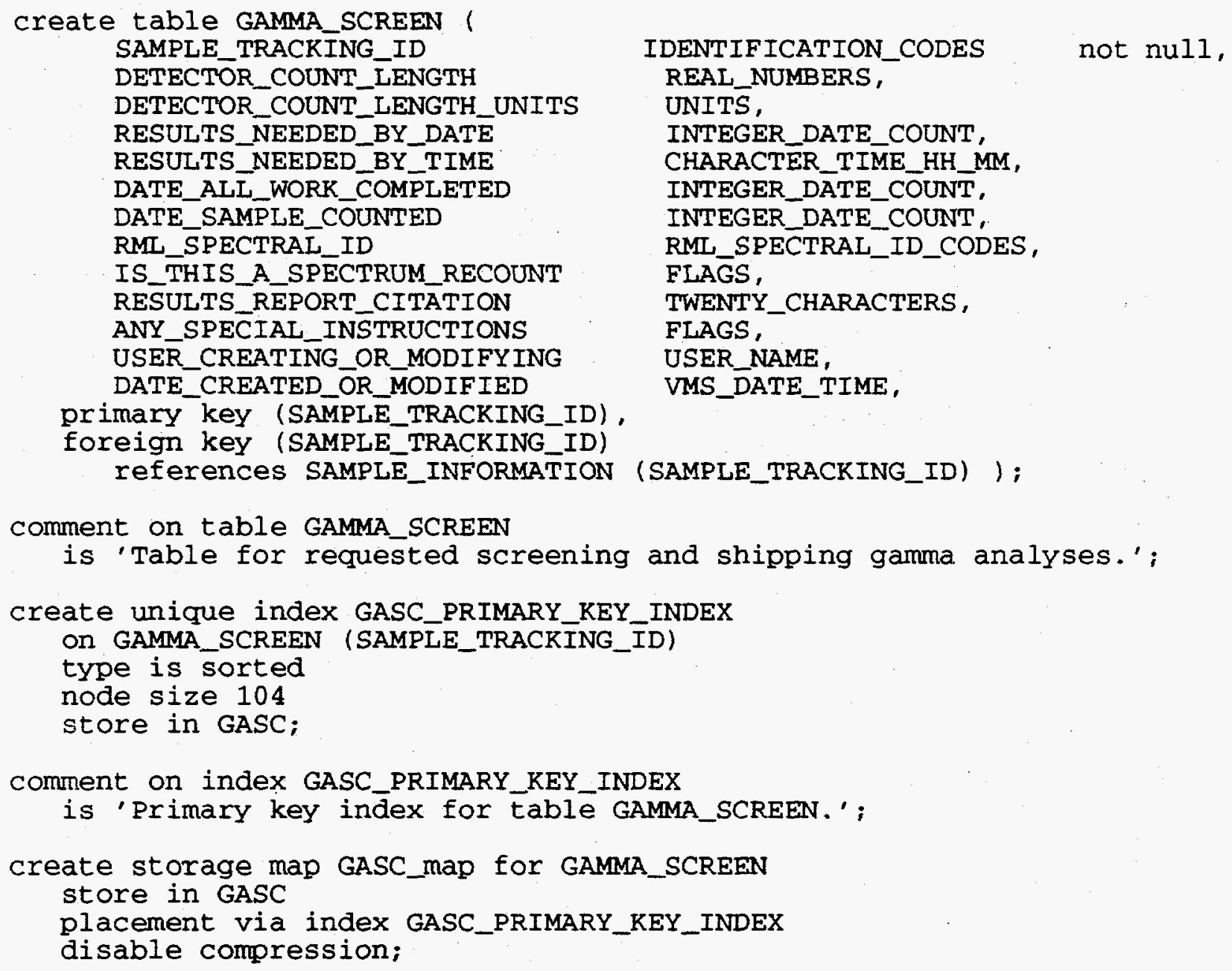


primary key (SAMPLE_TRACKING_ID),

foreign key (SAMPLE_TRACKING_ID)

references SAMPLE_INFORMATION (SAMPLE_TRACKING_ID) );

comment on table GAMMA_FULL_ISOTOPIC

is 'Table for requested full isotopic gamma analyses.';

create unique index GAFUIS_PRIMARY_KEY_INDEX

On GAMMA_FULL_ISOTOPIC (SAMPLE_TRACKING_ID)

type is sorted

node size 104

store in GAFUIS;

comment on index GAFUIS_PRIMARY_KEY_INDEX

is 'Primary key index for table GAMMA_FULL_ISOTOPIC.';

create storage map GAFUIS_map for GAMMA_FULL_ISOTOPIC

store in GAFUIS

placement via index GAFUIS_PRIMARY_KEY_INDEX

disable compression;

create table GAMMA_OTHER (

SAMPLE_TRACKING_ID

DETECTOR_COUNT_LENGTH

DETECTOR_COUNT_LENGTH_UNITS

RESULTS_NEEDED_BY_DATE

RESULTS_NEEDED_BY_TIME

DATE_ALI_WORK_COMPLETED

DATE_SAMPLE_COUNTED

RML_SPECTRAL_ID

IS_THIS_A_SPECTRUM_RECOUNT

RESULTS_REPORT_CITATION

ANY_SPECIAL_INSTRUCTIONS

USER_CREATING_OR_MODIFYING

DATE_CREATED_OR_MODIFIED

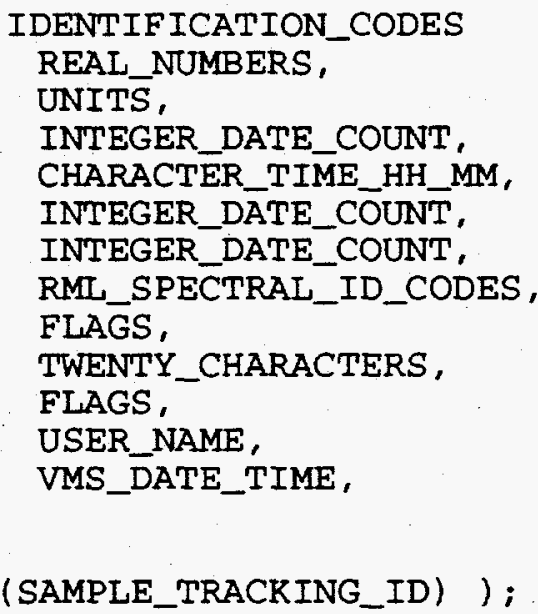

not null,

primary key (SAMPLE_TRACKING_ID)

foreign key (SAMPLE_TRACKING_ID)

references SAMPLE_INFORMATION (SAMPLE_TRACKING_ID) );

comment on table GAMMA_OTHER

is 'Table for requested gamma analyses for other, unlisted radionuclides.';

create unique index GAOT_PRIMARY_KEY_INDEX

On GAMMA_OTHER (SAMPLE_TRACKING_ID)

type is sorted

node size 104

store in GAOT;

comment on index GAOT_PRIMARY_KEY_INDEX

is 'Primary key index for table GAMMA_OTHER.';

create storage map GAOT_map for GAMMA_OTHER

store in GAOT

* placement via index GAOT_PRIMARY_KEY_INDEX

disable compression;

create table GROSS_ALPHA_BETA_AIR_FILTERS (

SAMPLE_TRACKING_ID

DETECTOR_COUNT_LENGTH

IDENTIFICATION_CODES not null, REAL_NUMBERS, 
DETECTOR_COUNT_LENGTH_UNITS

RESULTS_NEEDED_BY_DATE

RESULTS_NEEDED_BY_TIME

DATE_ALI_WORK_COMPLETED

RESULTS_REPORT_CITATION

ANY_SPECIAL_INSTRUCTIONS

USER_CREATING_OR_MODIFYING

DATE_CREATED_OR_MODIFIED

primary key (SAMPLE_TRACKING_ID),

foreign key (SAMPLE_TRACKING_ID)

references SAMPLE_INFORMATION
UNITS,

INTEGER_DATE_COUNT,

CHARACTER_TIME_HH_MM, INTEGER_DATE_COUNT, TWENTY_C CHARACTERS,

FLAGS,

USER_NAME,

VMS_DATE_TIME,

comment on tab_e GROSS_ALPHA_BETA_AIR_FILTERS

is 'Table for requested gross alpha-beta analyses of air filters.';

create unique index

on GROSS_ALPHA_BE... AIR_FILTERS (SAMPLE_TRACKING_ID)

type is sorted

node size 104

store in GRALBEAIFI;

comment on index GRALBEAIFI_PRIMARY_KEY_INDEX

is 'Primary key index for table GROSS_ALPHA_BETA_AIR_FILTERS.' ;

create storage map GRALBEAIFI_map for GROSS_ALPHA_BETA_AIR_FILTERS

store in GRALBEAIFI

placement via index GRALBEAIFI_PRIMARY_KEY_INDEX

disable compression;

create table GROSS_ALPHA_BETA_OTHER (

SAMPLE_TRACKING_ID

DETECTOR_COUNT_LENGTH

DETECTOR_COUNT_LENGTH_UNITS

RESULTS_NEEDED_BY_DATE

RESULTS_NEEDED_BY_TIME

DATE_ALI_WCAK_COMPLETED

RESULTS_REPORT_CITATION

ANY_SPECIAL_INSTRUCTIONS

USER_CREATING_OR_MODIFYING

DATE_CREATED_OR_MODIFIED

IDENTIFICATION_CODES

REAL_NUMBERS,

UNITS,

INTEGER_DATE_COUNT, CHARACTER_TIME_HH_MM,

TYTEGER_DATE_COUNT,

DENTY_CHARACTERS,

F. $\mathrm{MS}$,

USZR_NAME,

VMS_DATE_TIME,

primary key (SAMPLE_TRACKING_ID),

foreign key (SAMPLE_TRACKING_ID)

references SAMPLE_INFORMATION (SAMPLE_TRACKING_ID) );

comment on table GROSS_ALPHA_BETA_OTHER

is Table for requested gross alpha-beta analyses of other, unlisted samples.';

create unique index GRALBEOT_PRIMARY_KEY INDEX

On GROSS_ALPHA_BETA_OTHER (SAMPLE_TRACKING_ID)

type is sorted

node size 104

store in GRALBEOT;

comment on index GRALBEOT_PRIMARY_KEY_INDEX

is 'Primary key index for table GROSS_ALPHA_BETA_OTHER.' ;

create storage map GRALBEOT_map for GROSS_ALPHA_BETA_OTHER

store in GRALBEOT

placement via index GRALBEOT_PRIMARY_KEY_INDEX 
disable compression;

create table SPECIAL_INSTRUCTIONS ( SAMPLE_TRACKING_ID

ORIGIN_OF_SPECIAL_INSTRUCTION

SPECIAL_INSTTRUCTION

SPECIAL_INSTRUCTION_LINE

USER_CREATING_OR_MODIFYING

DATE_CREATED_OR_MODIFIED

primary key (SAMPLE_TRACKING_ID, ORIGIN_OF_SPECIAL_INSTRUCTION,

foreign key (SAMPLE_TRACKING_ID)

references SAMPLE_INFORMATION (SAMPLE_TRACKING_ID) );

comment on table SPECIAL_INSTRUCTIONS

is 'Table for special instructions.';

create unique index SPIN_PRIMARY_KEY_INDEX

On SPECIAL_INSTRUCTIONS (SAMPLE_TRACKING_ID, ORIGIN_OF_SPECIAL_INSTRUCTION，SPECIAL_INSTRUCTION_LINE)

type is sorted

node size 170

store in SPIN;

comment on index SPIN_PRIMARY_KEY_INDEX

is 'Primary key index for table SPECIAL_INSTRUCTIONS.';

create storage map SPIN_map for SPECIAL_INSTRUCTIONS

store in SPIN

placement via index SPIN_PRIMARY_KEY_INDEX

disable compression;

create table TRACKING (

SAMPLE_TRACKING_ID

POSSESSOR_SEQUENCE_NUMBER

POSSESSOR_NAME

POSSESSOR_PHONE_NUMBER

POSSESSOR_ADDRESS

POSSESSOR_POSSESSION_REASON

DATE_ACCEPTED_BY_POSSESSOR

DATE_RELINQUISHED_BY_POSSESSOR

USER_CREATING_OR_MODIFYING

DATE_CREATED_OR_MODIFIED

IDENTIFICATION_CODES

INTEGER_NUMBERS

not null,

NAMES,

PHONE_NUMBERS,

SIXTY_CHARACTERS,

FIFTY_CHARACTERS,

INTEGER_DATE_COUNT,

INTEGER_DATE_COUNT,

USER_NAME,

VMS_DATE_TIME,

primary key (SAMPLE_TRACKING_ID, POSSESSOR_SEQUENCE_NUMBER)，

foreign key (SAMPLE_TRACKING_ID)

references SAMPLE_INFORMATION (SAMPLE_TRACKING_ID) );

comment on table TRACKING

is 'Table for tracking samples.';

create unique index TR_PRIMARY_KEY_INDEX

on TRACKING (SAMPLE_TRACKING_ID, POSSESSOR_SEQUENCE_NUMBER)

type is sorted

node size 119

store in TR;

comment on index TR_PRIMARY_KEY_INDEX

is 'Primary key index for table TRACKING.';

create storage map TR_map for TRACKING 
store in TR

placement via index TR_PRIMARY_KEY_INDEX

disable compression;

commit;

exit; 


\section{E. BUILD_SCREEN - A ROUTINE TO CONSTRUCT FIELD LABELS AND LOCATIONS FOR DATA ENTRY SCREENS}

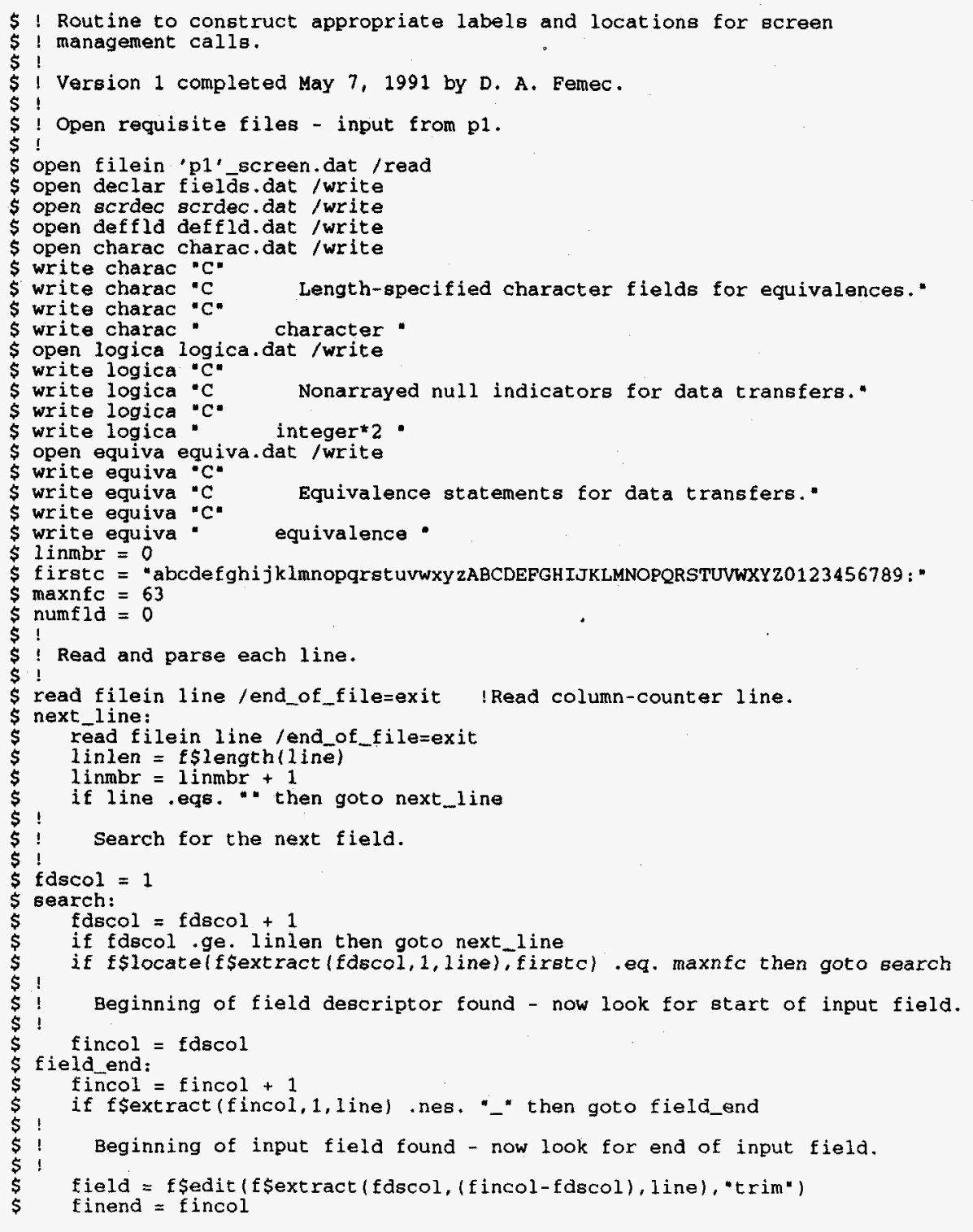




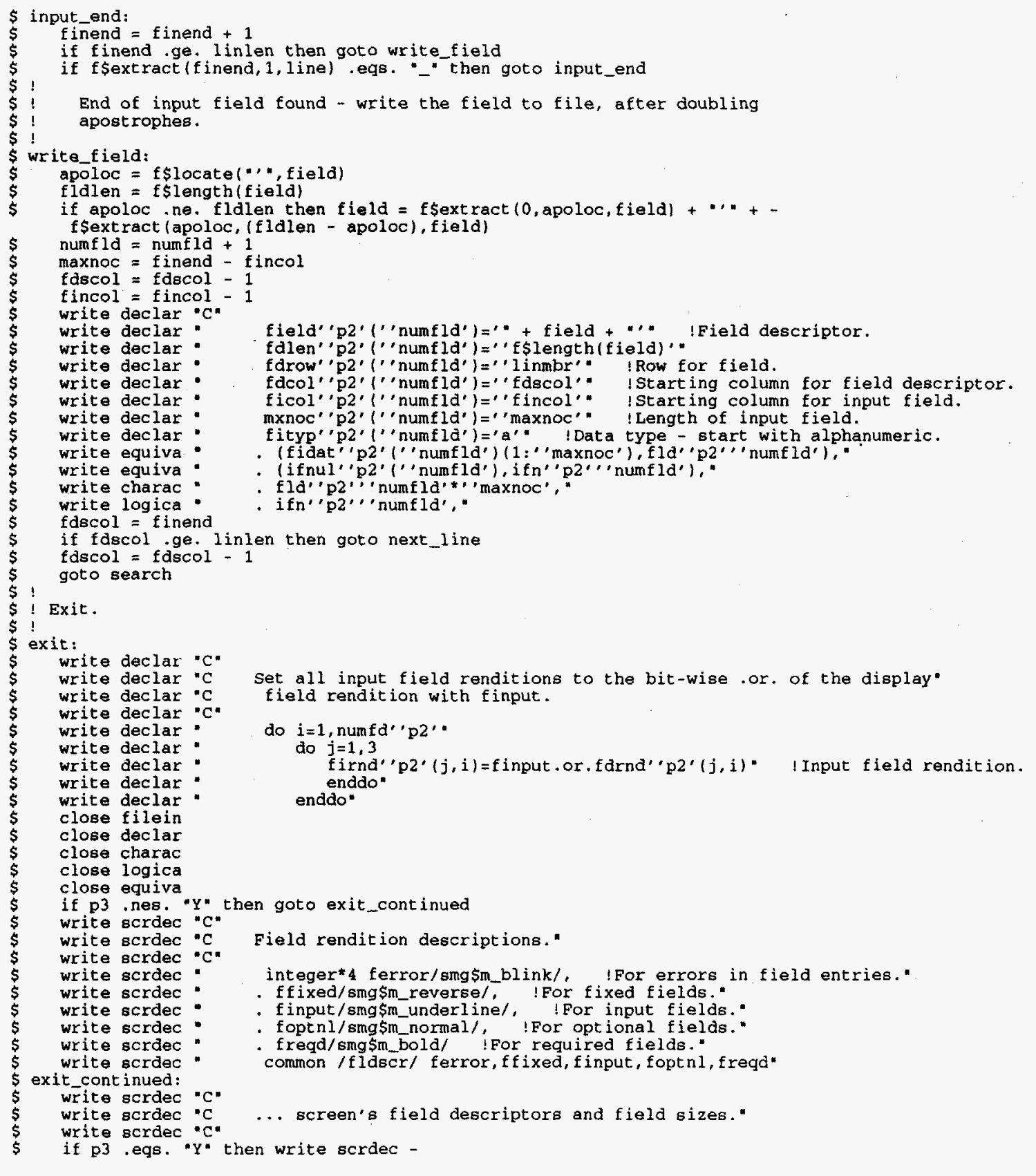


character*80 field" 'p2'("numfld'), fidat" $p 2$ '("numfld'),findat, blanks" s. "Y" then write $B c r d e c$

write character* 80 field" 'p2'(' 'numfld'), fidat' 'p2'('numfld').

write scrdec: character 30 atrbt"'p2,'(2,"'numfla')"

"P3 .eqs, "Y" then write scrdec -

"Yer" spaces(80)/80"', fityp" 'p2'('"numfld').

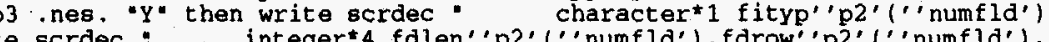

"numfld"), !Field renditions (add/update/search)."

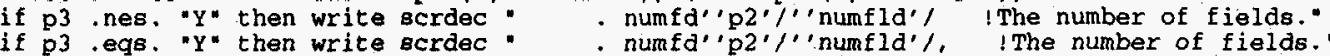

if p3 egs. "Y" then write scrdec " 2, ferror, ffixed, finput, foptnl, freqd !Field renditions."

write scrdec: integer* 2 ifnul' p2 (' numfld') ! Null indicator ( 1 if null, 0 otherwise) ".

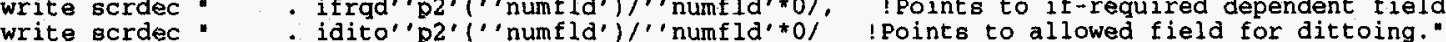

write scrdec : logical ifmod "p2'(" numfld') " Has a $\mathrm{field}$ been changed in an Update?"

common 'dbdat' 'p2' frield' 'p2', fidat' 'p2', fdlen' 'p2', fdrow' 'p2', fdcol' 'p2', ficol' 'p2', mxnoc' 'p2', '"

write scrdec : $\mathrm{y}$. then idito' 'p2 ':

if $\mathrm{p} 3$.eqs. "Y" then write scrdec

equivalence (blanks $(1: 1)$, spaces (1))"

Set defaults for display field renditions now so they can be changed

write deffld "C with each field as necessary."

write deffld " $\mathrm{C}$

write deffld

write deffld:

write deffld:

write deffld.

do $i=1$, numfd"'p2',

do $j=1,3$ ! 1 for adding, 2 for updating, and 3 for searching.

$2^{\prime}(j, i)=$ foptnl "

close scrdec

enddo"

close deffld

copy/concatenate scrdec.dat + charac.dat + logica.dat + equiva.dat -

delete scrdec.dat; , fields.dat; , charac.dat; , logica.dat; , equiva.dat; , deffld.dat; 


\section{F. SCREEN TEMPLATE FOR THE GROSS ALPHA-BETA ANALYSES SCREEN SUBMITTED TO BUILD_SCREEN}

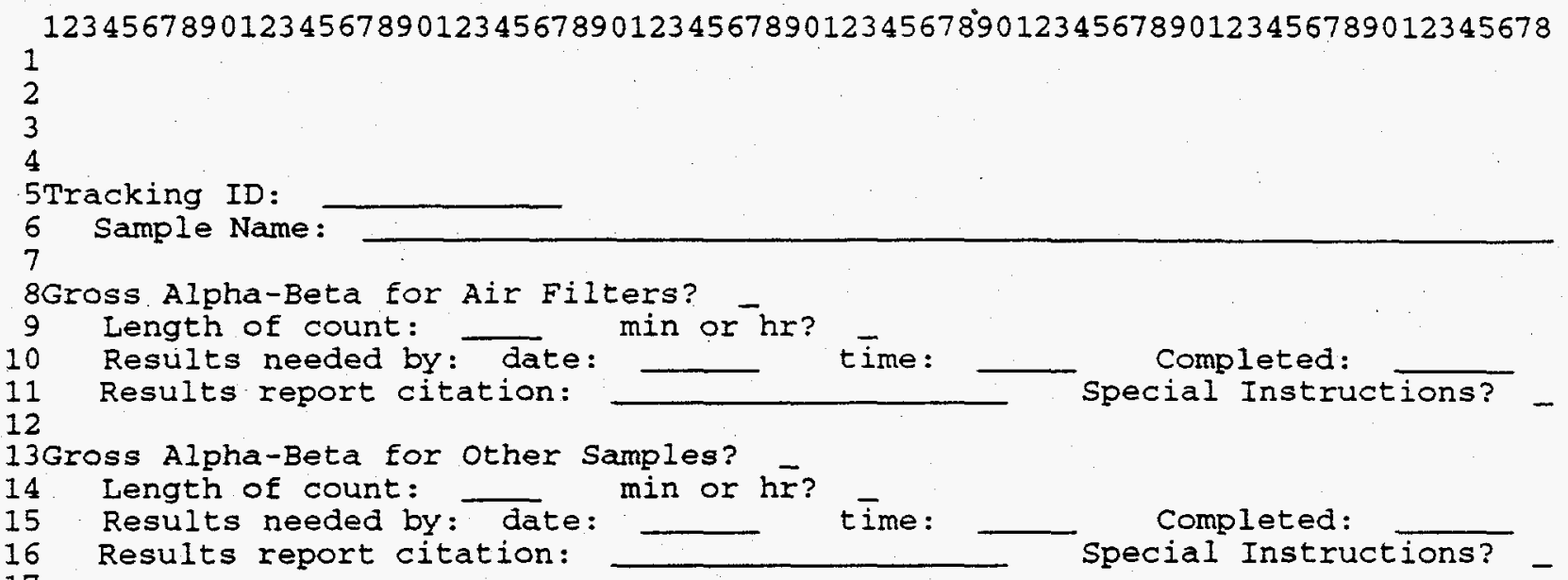




\section{G. BUILD_SCREEN OUTPUT FOR THE GROSS ALPHA-BETA ANALYSES SCREEN}

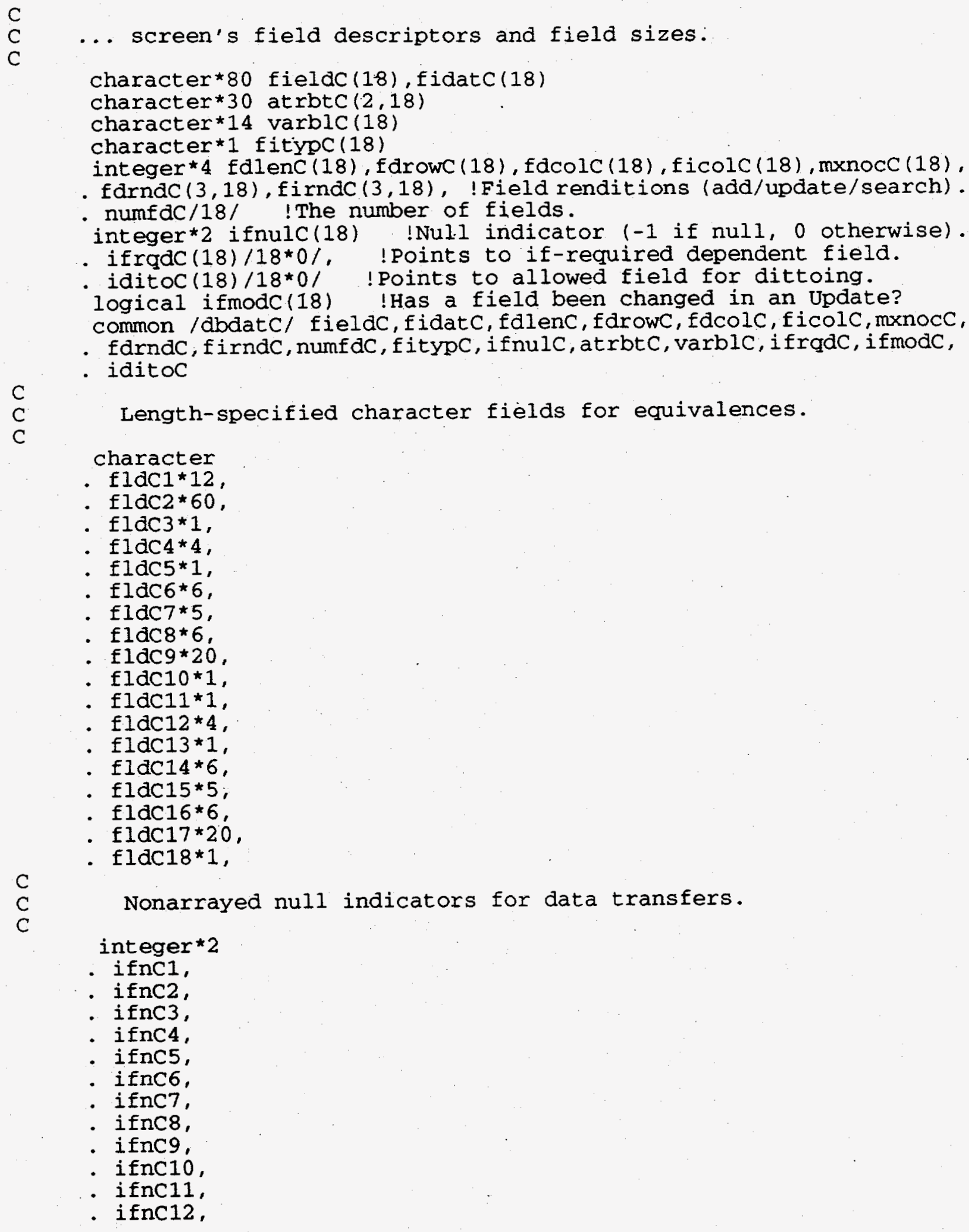

C

C $\quad$ C.. screen's field descriptors and field sizes:

character $* 80$ fieldC (18), fidatc(18)

character $* 30$ atrbtc $(2,18)$

character*14 varblc(18)

character*1 fitypc(18)

integer 4 fdlenc(18), farowC (18), fdcolc (18), ficolc (18), mxnocC (18).

- fdrnac $(3,18)$, firndC $(3,18)$, ! Field renditions (add/update/search).

- numfaC $/ 18 /$ ! The number of fields.

integer*2 ifnulC(18) !Null indicator (-1 if null, 0 otherwise).

- ifrqaC (18)/18*0/, ! Points to if-required dependent field.

- iditoc (18)/18*0/ ! Points to allowed field for dittoing.

logical ifmodC(18) !Has a field been changed in an Update? common /dbdatc/ fieldC, fidatc, falenc, fdrowC, facolc, ficolc, mxnocc, - farndC, firndC, numfdC, fitypc, ifnulc, atrbtc, varblc, ifrqdC, ifmodC,

- iditoc

$\mathrm{C}$
$\mathrm{C}$
$\mathrm{C}$ Length-specified character fields for equivalences.

character

- fldC $1 * 12$,

- fldC2*60,

- $\mathrm{fldC} 3 * 1$,

- $\mathrm{fldC} 4 * 4$

- $\mathrm{I} 1 \mathrm{dC} 5 * 1$,

- flac6*6,

- fldC $7 * 5$,

- flaC 8 *6.

- EldC9*20,

- fldc10*1,

- fldc $11 * 1$,

- flac $12 * 4$,

- $\mathrm{f} 1 \mathrm{dC} 13 * 1$,

- flaC14*6,

- flac $15 * 5$;

- flac $16 * 6$,

- flac17*20,

- fldC18*1,

$\mathrm{C}$
$\mathrm{C}$ Nonarrayed null indicators for data transfers.

integer *2

- ifnc1,

- ifnc2,

- ifnc3,

- ifnc4,

- ifnC5,

- ifnc6.

- ifnc7,

- ifnc8,

- ifnc9.

- ifnc10,

- ifnC11,

- ifnc12, 


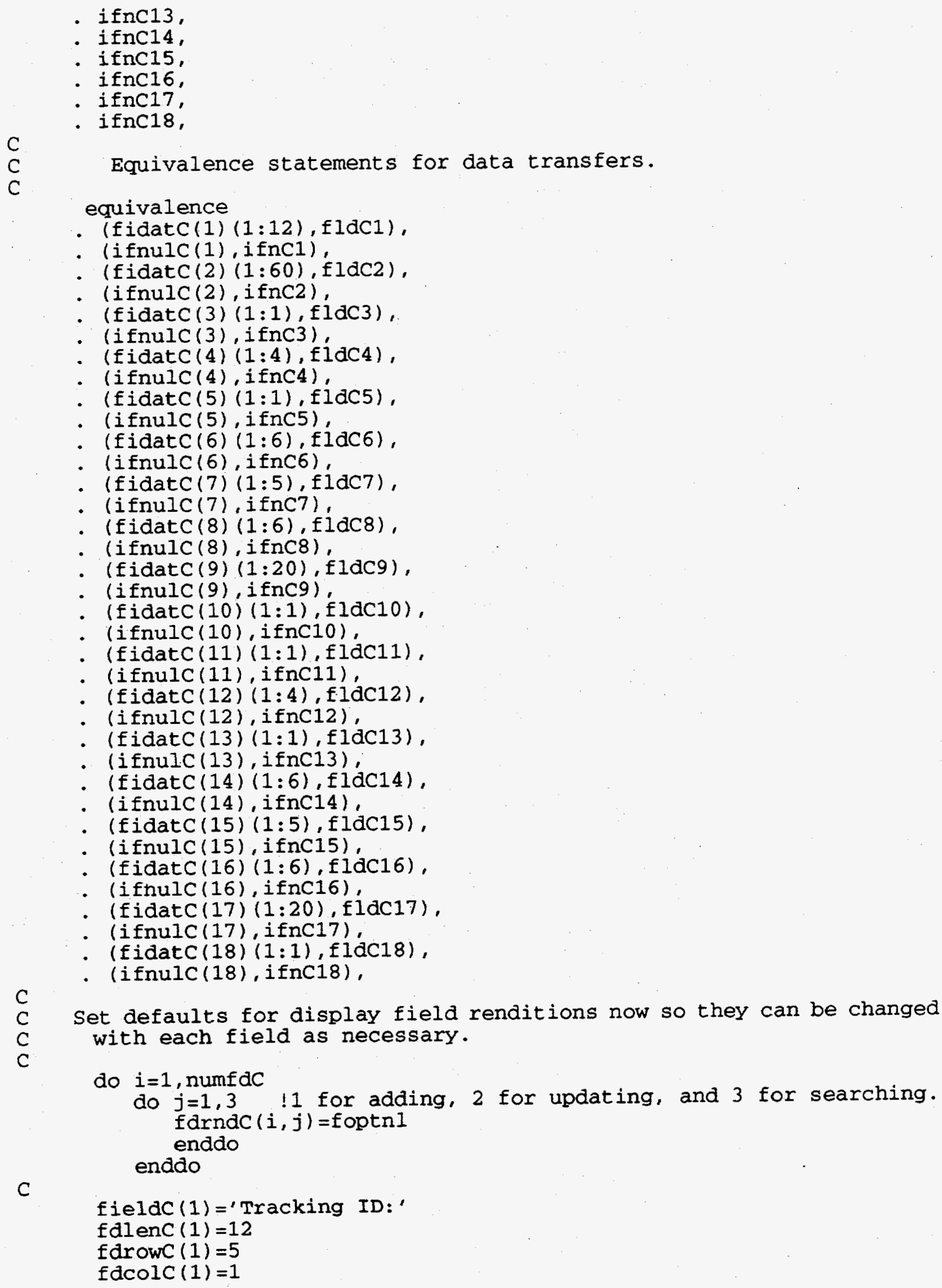

C

C

C

Set defaults for display field renditions now so they can be changed with each field as necessary.

do $i=1$, numfdC

do $j=1,3$ ! 1 for adding, 2 for updating, and 3 for searching. fdrndc $(i, j)=$ foptnl enddo

enddo

C

fieldC $(1)=$ 'Tracking ID: '

falenc $(1)=12$

fdrowC $(1)=5$

fdcolc $(1)=1$ 
ficolc $(1)=15$

$\operatorname{mxnocC}(1)=12$

C

fitypc $(1)=' a$ '

fieldc $(2)=$ 'Sample Name:"

fdlenc $(2)=12$

fdrowC $(2)=6$

fdcolc $(2)=4$

ficolc $(2)=18$

$\operatorname{mxnocC}(2)=60$

C

fitypc $(2)=' a$ '

fieldC (3) ='Gross Alpha-Beta for Air Filters?'

fdlenc $(3)=33$

fdrowC $(3)=8$

fdcolc $(3)=1$

$\mathrm{ficolc}(3)=36$

$\operatorname{mxnocC}(3)=1$

C

fitypc $(3)=' a$ '

fieldC $(4)=$ 'Length of count:'

fdlenc $(4)=16$

farowC $(4)=9$

$\mathrm{fdcolC}(4)=4$

$\mathrm{ficolc}(4)=22$

$\operatorname{mxnocC}(4)=4$

C

fitypc $(4)=' a$ '

fieldC $(5)=' \min$ or $h r$ ?'

falenc $(5)=10$

farowC $(5)=9$

fdcolc $(5)=30$

$\mathrm{ficolC}(5)=42$

$\operatorname{mxnocC}(5)=1$

C

fitypc $(5)=' a^{\prime}$

fieldC $(6)=$ 'Results needed by: date:'

falenc $(6)=25$

fdrowC $(6)=10$

facolc $(6)=4$

$\mathrm{ficolc}(6)=31$

$\operatorname{mxnocC}(6)=6$

C

fitypC $(6)=' a^{\prime}$

fieldc $(7)=$ 'time:'

falenc $(7)=5$

fdrowC $(7)=10$

fdcolc $(7)=41$

$\mathrm{ficolc}(7)=48$

$\operatorname{mxnocC}(7)=5$

C

fitypc $(7)=a^{\prime}$

fieldC $(8)=$ ' Completed:'

fdlenc $(8)=10$

farowc $(8)=10$

fdcolc $(8)=57$

ficolc $(8)=69$

$\operatorname{mxnocC}(8)=6$

C

fitypc $(8)=$ ' $a$ ' 
C

fieldc $(9)=$ 'Results report citation:'

fdlenc $(9)=24$

fdrowc $(9)=11$

fdcolc $(9)=4$

ficolc $(9)=30$

$\operatorname{mxnocC}(9)=20$

fitypc $(9)=' a$ '

fieldc $(10)=$ 'Special Instructions?'

fdlenc $(10)=21$

farowC $(10)=11$

fdcolc $(10)=54$

ficolc $(10)=77$

$\operatorname{mxnocC}(10)=1$

C

fitypC $(10)=' a$ '

fieldC (11)='Gross Alpha-Beta for Other Samples?'

fdlenc $(11)=35$

fdrowc $(11)=13$

$\operatorname{fdcolc}(11)=1$

ficolc $(11)=38$

$\operatorname{mxnocC}(11)=1$

C

fitypc $(11)=$ 'a'

fieldC $(12)=$ 'Length of count:'

fdlenc $(12)=16$

fdrowC $(12)=14$

fdeolc $(12)=4$

ficolc $(12)=22$

$\operatorname{mxnocC}(12)=4$

C

fitypc $(12)=' a$ '

fieldc (13) ='min or hr?'

fdlenc $(13)=10$.

farowC $(13)=14$

fdcolc $(13)=30$

ficolc $(13)=42$

$\operatorname{mxnocC}(13)=1$

$\mathrm{C}$

fitypc $(13)=' a$ '

fieldc $(14)=$ 'Results needed by: date:'

fdlenc $(14)=25$

fdrowC $(14)=15$

$\operatorname{fdcolC}(14)=4$

ficolc $(14)=31$

$\operatorname{mxnocC}(14)=6$

C

fitypc $(14)=$ ' $a$ '

fieldc $(15)=$ 't ime:'

fdlenc $(15)=5$

fdrowC $(15)=15$

fdcolc $(15)=41$

ficolc $(15)=48$

$\operatorname{mxnocC}(15)=5$

C

fitypc $(15)=$ 'a'

fieldc $(16)=$ 'Completed:'

fdlenc $(16)=10$

fdrowc $(16)=15$

fdcolc $(16)=57$ 


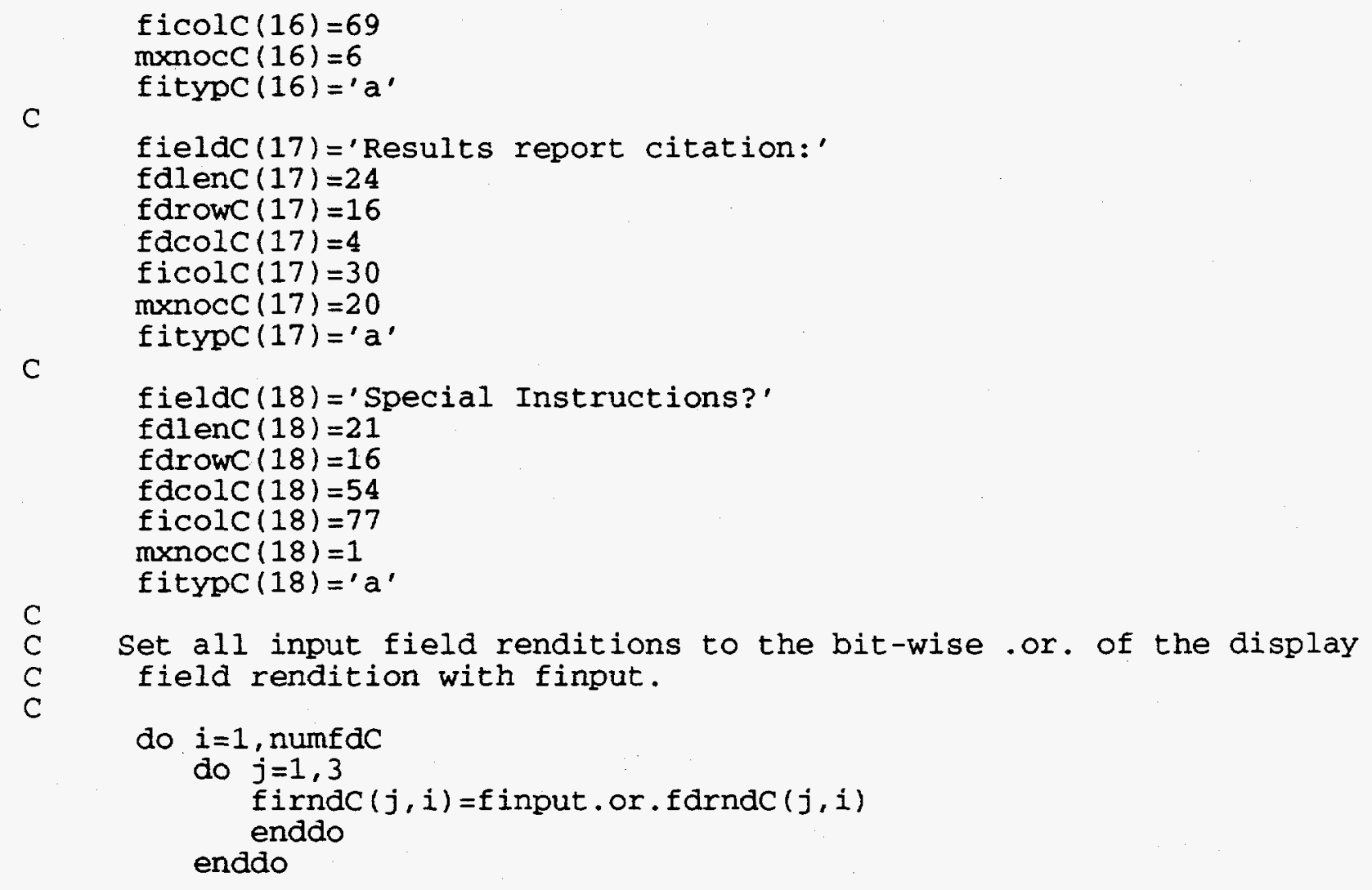




\section{H. ARRAY AND SCALAR DECLARATIONS RELATED TO THE SCREEN MANAGEMENT ROUTINES}

$C$
$C$
$C$
$C$
$C$
$C$
$C$
$C$
$C$
$C$
$C$

C

C

C
C
C

C
C
C

C
C
C
C

Declaration statements.

Component of SAMPLE_TRACKING Version 1 completed June 6, 1991 by D. A. Femec.

Note that variable names are limited to six-characters in length; this is a limitation imposed by SQL for variables used in SQL calls.

Field rendition descriptions.

integer*4 ferror/smg\$m_blink/, lFor errors in field entries.

- ffixed/smg\$m_reverse/, !For fixed fields.

- finput/smg\$m_underline/, ! For input fields.

- foptnl/smg\$m_normal/, !For optional fields.

- freqd/smg\$m_bold/ !For required fields.

common /fldscr/ ferror, ffixed, finput, foptnl, freqd

Main screen's field descriptors and field sizes.

character $\star 80$ fieldM (36), fidatM (36), findat,blanks

character $\star 30$ at rbtM $(2,38)$

character*14 varblM(38)

character* 1 spaces (80)/80*' /,fitypM (36)

integer 4 fdlenM (36), fdrowm (36), fdcolm $(36), \operatorname{ficolm}(36), \operatorname{mxnocm}(36)$,

- fdrndM $(3,36)$, firndM $(3,36)$, ! Field renditions (add/update/search).

- numfdM/36/ !The number of fields.

integer*2 ifnulM(36), !Null indicator (-1 if null, 0 otherwise).

- ifrgdM(36) $/ 36 * 0 \%$ ! Points to if-required dependent field.

- iditom(36)/36*0/ ! Points to allowed field for dittoing.

logical ifmodm(36) ! Has a field been modified in an Update?

common /dbdatM/ fieldM, fidatM, fdlenM, fdrowM, fdcolM, ficolM, mxnocM,

- fdrndM, firndM, numfdM, fitypM, ifnulm, atrbtM, varblM, ifrqdM, i fmodM,

- iditom

equivalence (blanks (1:1), spaces (1))

Length-specified character fields for equivalences ( $f$ for field).

character fMSTI 12, fMDSE* 6, fMDAWC *6, fMCSI * 12, fMRNBD *6, fMCPN 40 ,

- fMWCN $* 9, f M C S N * 60, f M S T * 10, \mathrm{fMSS} * 5, \mathrm{fMSSU} * 5, \mathrm{fMSCD} * 6, \mathrm{fMSCT} * 5, \mathrm{fMASI} * 1$,

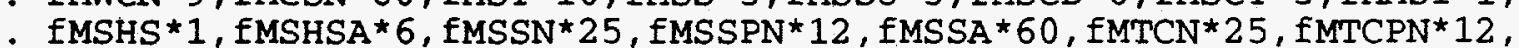

- fMTCA $* 60, f M S R T N * 25$, fMSRTP $* 12$, fMSRTA $* 60$, fMSPBN ${ }^{\star} 25$, fMSPBP $* 12$,

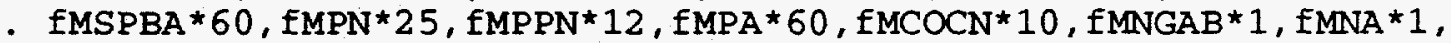

- $\mathrm{fMNB}^{\star} 1, \mathrm{fMNG}{ }^{\star} 1$

Real and integer field storage ( $r$ for real, $i$ for integer).

real* 4 rMSS, rMSHSA

integer * 4 iMDSE, iMDAWC, iMRNBD, iMSCD

- .nxinst, nxcust !Declared in the main routine.

Nonarrayed field lengths (1 for lengths).

integer *4 1MSTI, 1MDSE, IMDAWC, 1MCSI , IMRNBD, IMCPN, IMWCN, IMCSN, IMST, - IMSS, IMSSU, IMSCD , IMSCT , 1MASI , IMSHS, IMSHSA, IMSSN, IMSSPN, IMSSA, 
1MTCN, IMTCPN, 1MTCA, 1MSRTN, IMSRTP, 1MSRTA, 1MSPBN, IMSPBP, IMSPBA, IMPN, IMP PN, IMPA, IMCOCN, IMNGAB, IMNA, IMNB, IMNG

Nonarrayed null indicators for data transfers ( $n$ for null).

integer *2 nMSTI, nMDSE, nMDAWC, nMCSI, nMRNBD, nMCPN, nMWCN, nMCSN, nMST,

. nMSS, nMSSU, nMSCD , nMSCT, nMASI, IMSHS, nMSHSA, nMSSN, nMSSPN, nMSSA, nMTCN, nMTCPN, nMTCA, nMSRTN, IMSRTP , nMSRTA, nMSPBN, nMSPBP, nMSPBA,

- nMPN, nMP PN, nMPA, nMCOCN, nMNGAB, nMNA, nMNB, nMNG

C

Nonarrayed if-modified indicators ( $m$ for modified).

integer *2 mMSTI, MMDSE, MMDAWC, MMCSI, MMRNBD, MMCPN, MMWCN, MMCSN, MMST, mMSS , mMSSU, MMSCD , MMSCT, IMMASI, mMSHS, MMSHSA, MMSSN, MMSSPN, MMSSA,

- MMTCN, IMTCPN, MMTCA, MMSRTN, MMSRTP , MMSRTA, MMSPBN , MMSPBP , MMSPBA,

- MMPN , MMPPN, IMMPA, MMCOCN, MMNGAB, MMNA, IMNB , IMMNG

$c$
C
C

\section{Equivalence statements for data transfers.}

equivalence (fidatM(1) ( $1: 12$ ), fMSTI), (ifnulM(1), nMSTI),

(fidatM (2) $(1: 6)$, fMDSE), (ifnulM (2), nMDSE),

- (fidatm (3) $(1: 6)$, IMDAWC), (ifnulm(3), nMDAWC).

- (fidatm(4) (1:12), fMCSI), (ifnulm(4), nMCSI),

- (fidatm(5) $(1: 6)$, fMRNBD), (i fnulm(5), nMRNBD),

- (fidatM $(6)(1: 40)$, fMCPN), (ifnulM(6), nMCPN),

- (fidatM(7) (1:9), fMWCN), (ifnulM(7), nMWCN),

- (fidatm $(8)(1: 60), \operatorname{fMCSN}),($ ifnulM( 8$)$, nMCSN),

- (fidatm(9) (1:10), fMST), (ifnulM(9), nMST),

- (fidatm(10) (1:5), EMSS), (ifnulM(10), nMSS),

- (EidatM(11) (1:5), fMSSU), (ifnulM(11), nMSSU),

- (fidatM (12) (1:6), fMSCD), (i fnulm(12), nMSCD),

- (fidatm (13) (1:5), fMSCT), (ifnulm(13), nMSCT),

- (Eidatm (14) (1:1), fMASI), (i fnulM(14), nMASI),

- (fidatm(15) (1:1), fMSHS), (ifnulm(15), nMSHS),

- (fidatm (16) (1:6), fMSHSA), (ifnulm(16), nMSHSA),

- (fidatM (17) (1:25), fMSSN), (ifnulM(17), nMSSN),

- (fidatm(18) (1:12), fMSSPN), (i fnulM(18), nMSSPN),

- (fidatm (19) (1:60), fMSSA), (ifnulm(19), nMSSA),

- (fidatm (20) $(1: 25)$, fMTCN), (i $\operatorname{fnulM}(20), \mathrm{nMTCN}$ ),

- (fidatM(21) (1:12), fMTCPN), (ifnulM(21), nMTCPN),

- (fidatM (22) (1:60), fMTCA), (ifnulM(22), nMTCA),

- (EidatM(23) (1:25), fMSRTN), (ifnulM(23), nMSRTN),

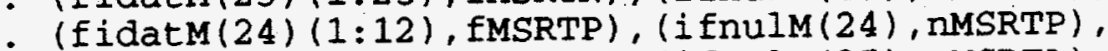

- (fidatm(25) (1:60), EMSRTA), (i fnulm(25), nMSRTA),

$\therefore$ (fidatm $(26)(1: 25)$, EMSPBN), (i fnulm(26), nMSPBN),

- (fidatm(27) (1:12), EMSPBP), (i fnulm(27), nMSPBP),

- (fidatm(28) (1:60), fMSPBA), (i fnulm(28), nMSPBA),

- (fidatM(29) (1:25), fMPN), (ifnulM(29), nMPN),

- (fidatm $(30)(1: 12)$, fMPPN), (ifnulm(30), nMPPN),

- (fidatm(31) (1:60), fMPA), (i fnulm(31), nMPA),

- (fidatm(32) (1:10), EMCOCN), (ifnulM(32), nMCOCN),

- (fidatm(33) (1:1), fMNGAB), (ifnulm(33), nMNGAB),

- (fidatM (34) (1:1), fMNA), (ifnulM (34), nMNA),

- (fidatm (35) (1:1), fMNB), (ifnulM (35), nMNB),

- (fidatm (36) (1:1), fMNG), (ifnulM (36), nMNG),

- (mxnocm (1), IMSTI), (i $\operatorname{fmodM}(1), \operatorname{mMSTI})$,

$\because(\operatorname{mxnocM}(2), 1 \mathrm{MDSE})$, (i $\operatorname{fmodM}(2), \operatorname{mMDSE})$,

- (mXnocM (3), IMDAWC), (ifmodM (3), mMDAWC),

- (mxnocm (4), 1MCSI), (ifmodM(4), mMCSI),

- (mXnocm (5), IMRNBD), (ifmodM (5), MMRNBD), 
(mxnocM $(6), 1 M C P N),(i \operatorname{fmodM}(6), \operatorname{mMCPN})$,

(mxnocm ( 7), 1MWCN), (i fmodM (7), mMWCN),

- (mXnocM $(8), 1 M C S N),(i \operatorname{fmodM}(8), \operatorname{mMCSN})$,

- (mxnocm (9), IMST), (ifmodM(9), mMST),

- (mXnocm(10), IMSS), (i fmodm(10), mMSS),

- (mxnocM(11), 1MSSU), (ifmodM(11), mMSSU),

- (mxnocm (12), IMSCD), (ifmodM (12), mMSCD),

- (mxnocM(13), IMSCT), (ifmodM (13), mMSCT),

- (mXnocm(14), 1MASI), (i $\operatorname{fmodM}(14)$, mMASI),

- (mxnocm(15), 1MSHS), (i fmodM (15), mMSHS),

- $(\operatorname{mxnOCM}(16), 1 \mathrm{MSHSA}),(i \operatorname{imodM}(16), \operatorname{mMSHSA})$,

- (mxnocm (17), IMSSN), (i $\operatorname{fmodM}(17), \operatorname{mMSSN})$,

- (mxnocM(18), 1MSSPN), (i $\operatorname{fmodM}(18), \operatorname{mMSSPN})$,

- (mxnocM (19), IMSSA), (i $\operatorname{modM}(19)$, mMSSA).

- (mXnOCM $(20), I M T C N),(i \operatorname{modM}(20), \operatorname{mMTCN})$,

- (mXnOcM(21), IMTCPN), (ifmodM $(21), \operatorname{mMTCPN})$,

- (mXnocM(22), 1MTCA), (i $\operatorname{modM}(22)$, mMTCA),

- (mXnOcM (23), IMSRTN) , (i $\operatorname{fmodM}(23)$, mMSRTN) ,

- (mXnOcM(24), IMSRTP), (i $\operatorname{fmodM}(24), \operatorname{mMSRTP})$,

- (mXnOcM (25), IMSRTA), (i $\operatorname{fmodM}(25)$, mMSRTA),

- $(\operatorname{mxnOcM}(26), 1 M S P B N),(i \operatorname{imodM}(26), \operatorname{mMSPBN})$,

- $(\operatorname{m\times nOCM}(27), 1 \mathrm{MSPBP})$, (i $\operatorname{fmodM}(27), \operatorname{mMSPBP}$ ),

- (mXnOCM (28), IMSPBA), (i $\operatorname{modM}(28), m M S P B A)$,

- (mxnocM(29), IMPN), (i $\operatorname{fmodM}(29), \operatorname{mMPN})$,

- $(\operatorname{mxnocM}(30), 1 M P P N),(i \operatorname{imodM}(30), \operatorname{mMPPN})$,

- (mxnocm(31), IMPA), (i fmodM(31), mMPA),

- (mxnOcM (32), IMCOCN), (i $\bmod (32), \operatorname{mMCOCN})$,

- (mxnocm (33), IMNGAB), (ifmodM (33), mMNGAB),

- (mXnocm (34), 1MNA), (i $\operatorname{fmodM}(34)$, mMNA),

- (mxnocm (35), IMNB), (i $\operatorname{fmodM}(35)$, mMNB),

- (mxnocm (36), IMNG), (ifmodM(36), mMNG)

C

C

Database table and attribute names for screen fields.

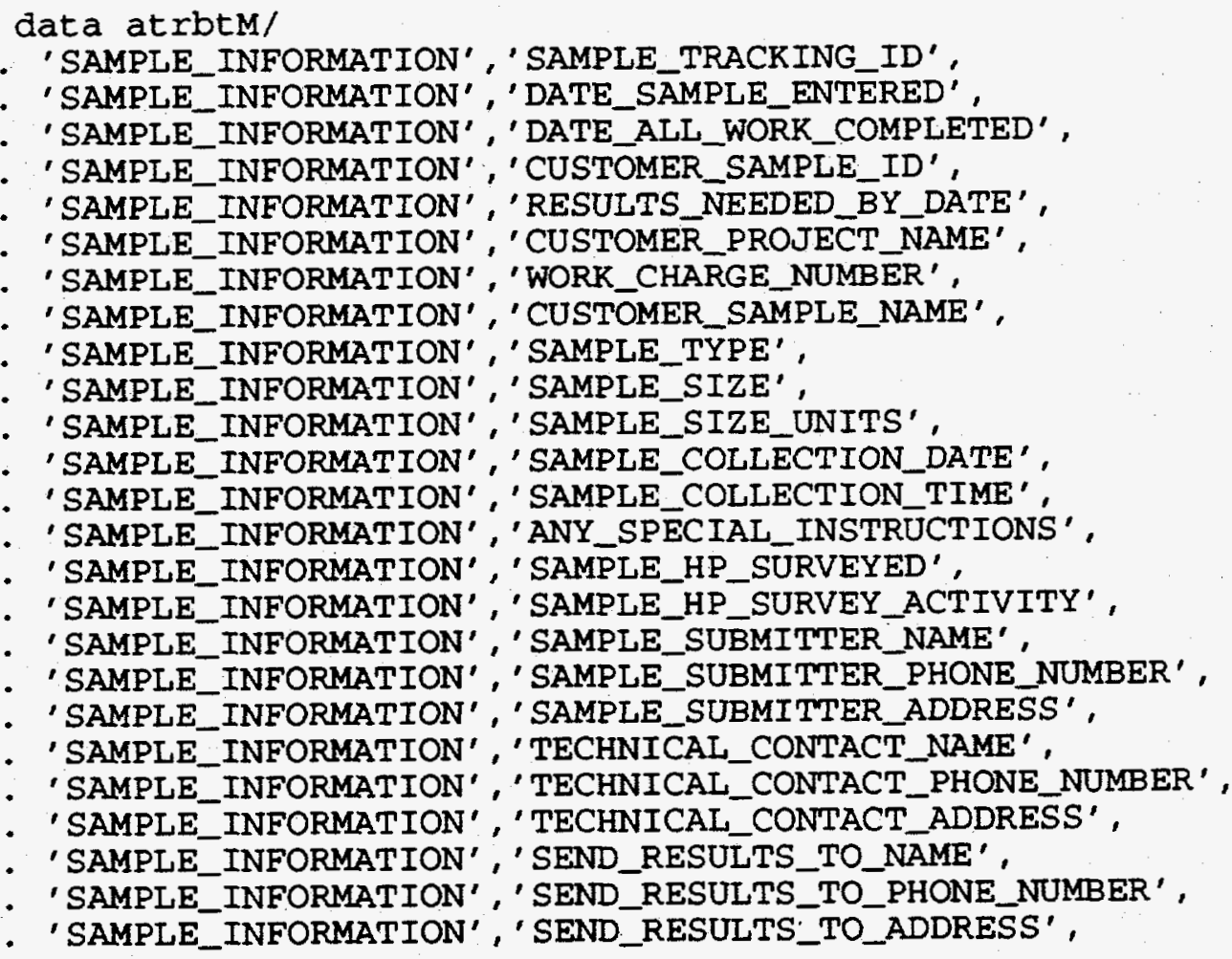




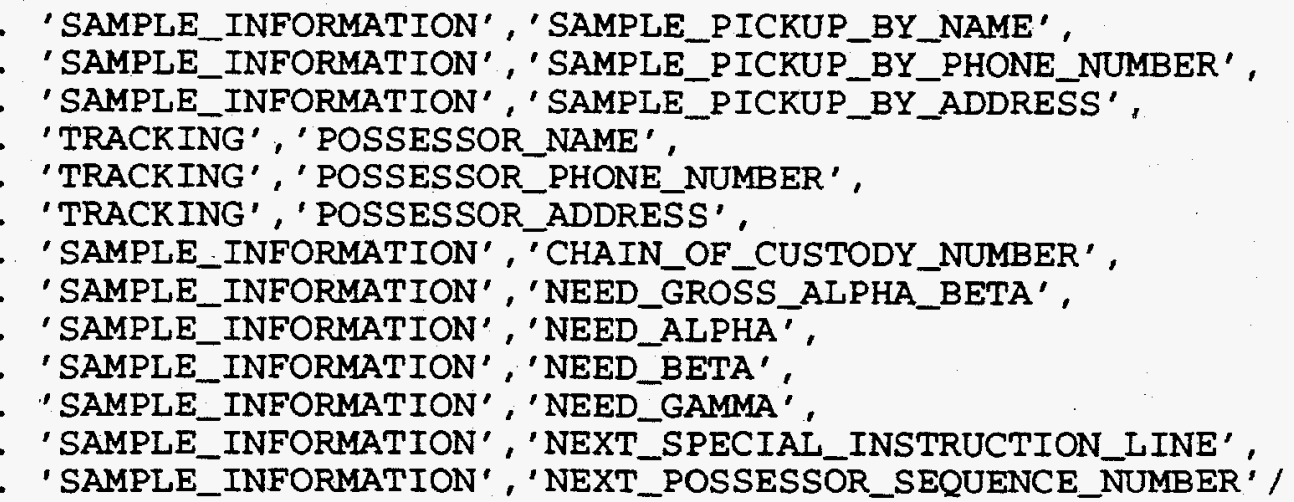

$\mathrm{C}$

C C

C C C

$\mathrm{C}$ C C

Program variable names (value and null indicator) for screen fields.

data varblM/': fMSTI:nMSTI',': iMDSE:nMDSE',': iMDAWC: nMDAWC',

' : EMCSI: nMCSI', ' : IMRNBD: nMRNBD', ' : fMCPN: nMCPN' ,' : IMWCN: $N M W C N '$,

' f MCSN:nMCSN', ' : fMST:nMST',' : rMSS:nMSS',' :fMSSU : nMSSU',

' : iMSCD:nMSCD' ,' : fMSCT:nMSCT',' : fMASI:nMASI' ,':fMSHS :nMSHS',

$'$ :rMSHSA:nMSHSA' , ': fMSSN:nMSSN', ' : fMSSPN: nMSSPN', ':fMSSA:nMSSA',

' : fMTCN : $n M T C N '$, ' : fMTCPN : nMTCPN', ' : fMTCA : nMTCA', ' : fMSRTN : nMSRTN',

' : fMSRTP:nMSRTP', ' : EMSRTA:nMSRTA' , : IMSPBN:nMSPBN',

$'$ : fMSPBP : nMSPBP', ' : fMSPBA: nMSPBA', ' : fMPN: nMPN',

' : IMPPN: nMPPN',' : fMPA : nMPA' ,' : fMCOCN:nMCOCN', ' : fMNGAB: nMNGAB' ,

$'$ : fMNA :nMNA',': fMNB : nMNB',' : fMNG :nMNG',' :nxinst : ifnnxi',

': nxcust : ifnnxc'/

Alpha screen's field descriptors and field sizes.

character $* 80$ fieldA (37), fidatA (37)

character $\star 30$ atrbtA $(2,37)$

character $* 14$ varbla (37)

character $* 1$ fitypa (37)

integer *4 fdlenA (37), fdrowA (37), fdcola (37), ficolA (37), mxnocA (37),

- fdrndA $(3,37)$, firnda $(3,37)$, ! Field renditions (add/update/search).

- numfdA/37/ !The number of fields.

integer*2 ifnulA(37), !Null indicator (-1 if null, 0 otherwise).

- ifrgda (37) $/ 37 * 0 \%$, Points to if-required dependent field.

- iditoA(37)/37*0/ ! Points to allowed field for dittoing.

logical ifmodA(37) ! Has a field been modified in an Update?

common /dbdatA/ fieldA, fidatA, fdlenA, fdrowA, fdcolA, ficolA, mxnocA,

- fdrndA, firndA, numfdA, fitypA, ifnulA, atrbtA, varblA, ifrgdA, i fmodA,

- iditoA

Length-specified character fields for equivalences (f for field).

character fASTI*12, £ACSN*60, fANAU*1, fARNB1*6, fADAW1*6, fARRC1*20,

. fAASI $1 * 1$, fANATh $* 1$, fARNB $2 * 6$, fADAW2 $* 6$, fARRC $2 * 20$, fAASI $2 * 1$, fANAPu $* 1$,

- fARNB $3 * 6$, fADAW $3 * 6$, fARRC $3 * 20$, fAASI $3 * 1$, fANASP $* 1$, fARNB 4 * 6, fADAW4 46 ,

- fARRC $4 * 20$, fAASI $4 * 1$, fANAWP * 1 , fARNB $5 * 6$, fADAW5 $* 6$, fARRC $5 * 20$, fAASI $5 * 1$,

- fANATS 1 , fARNB 6 *6, fADAW6*6, fARRC 6 *20, fAASI $6 * 1$, fANAO* 1 , fARNB7 6 ,

- EADAW7 76, EARRC $7 * 20$, fAASI $7 * 1$

Integer field storage ( $i$ for integer).

integer*4 iARNB1, iADAW1, iARNB2, iADAW2, iARNB3, iADAW3, iARNB4, iADAW4, IARNB5, IADAW5, IARNB6, IADAW6, IARNB7, iADAW7

Nonarrayed maximum field lengths ( 1 for length). 
integer *4 1ASTI, IACSN, IANAU, 1ARNB1, IADAW1, 1ARRC1, 1AAS I1, 1ANATh, - IARNB2, 1 ADAW2, IARRC2, IAASI2, IANAPU, IARNB3, IADAW3, IARRC3, IAASI3,

- IANASP, IARNB4, IADAW4, IARRC4, 1AASI4, IANAWP, 1 ARNB5, 1 ADAW5, IARRC5,

- IAASI5, IANATS, IARNB6, 1 ADAW6, 1 ARRC6, 1 AAS 16, 1 ANAO, 1 ARNB 7, 1 ADAW7,

- iARRC7, IAASI7

$$
\text { Nonarrayed null indicators for data transfers (n for null). }
$$

integer *2 nASTI, nACSN, nANAU, nARNB1, nADAW1, nARRC1, nAASI1, nANATh, - nARNB2, nADAW2, nARRC2, nAASI2, nANAPU, nARNB3, nADAW3, nARRC3, nAASI3, - nANASP, nARNB4, nADAW4, nARRC4, nAASI4, nANAWP, nARNB5, nADAW5, nARRC5, - nAASI5, nANATS, nARNB6, nADAW6, nARRC6, nAASI6, nANAO, nARNB7, nADAW7, - nARRC7, nAASI7

Nonarrayed if-modified indicators ( $m$ for modified).

integer *2 MASTI, MACSN, MANAU, MARNB1, MADAWI, MARRC1, MAASII, MANATh, - MARNB2 , MADAW2, MARRC2 , MAASI2, MANAPU, MARNB3, MADAW3, MARRC3, MAASI3, - MANASP, MARNB4, MADAW4, MARRC4, MAASI4, MANAWP, MARNB5, mADAW5, MARRC5, - MAASI5, mANATS, MARNB6, MADAW6, MARRC6, MAASI6, MANAO, MARNB7, MADAW7, - mARRC7, maAsI7

\section{Equivalence statements for data transfers.}

equivalence (fidata (1) (1:12), fASTI), (ifnula(1), nASTI), (fidata (2) $(1: 60)$, fACSN), (ifnulA (2), nACSN),

- (fidata (3) (1:1), fANAU), (ifnula (3), nANAU),

- (fidata (4) (1:6), farnB1), (ifnula (4), naRNB1),

- (fidata (5) $(1: 6)$, faDAW1), (ifnula (5), nADAW1), (fidata (6) (1:20), fARRC1), (ifnula (6), nARRC1),

- (fidata (7) $(1: 1)$, faAsI1), (i fnula (7),nAasI 1),

- (fidata (8) (1:1), faNATh), (ifnula (8), nANATh), (fidatA (9) $(1: 6)$, farNB2), (ifnula (9), nARNB2),

- (fidata (10) (1:6), fADAW2), (i fnula (10), nADAW2),

- (fidata (11) $(1: 20)$, fARRC2), (i fnula (11), nARRC2),

- (fidata (12) (1:1), fAASI2), (ifnula (12), nAASI2),

- (fidata (13) (1:1), faNAPu), (ifnula (13), nANAPu),

- (fidata (14) (1:6), faRnB3), (i fnula (14), nARNB3),

- (fidatA (15) (1:6), fADAW3), (ifnula (15), nADAW3),

- (fidata (16) (1:20), farRC3), (ifnula (16), nARRC3),

- (fidata (17) (1:1), faAsI3), (ifnula (17), naAsI3),

- (fidata (18) (1:1), fanasp), (ifnula (18), naNASP),

- (fidata (19) (1:6), farnB4), (ifnula (19), nARNB4),

- (fidata (20) (1:6), faDAW4), (i fnula (20), naDAW4),

- (fidata (21) $(1: 20)$, farRC4), (i fnula (21), nARRC4),

- (fidata (22) (1:1), faAsI4), (ifnula (22), nAASI4),

- (fidata (23) (1:1), fanAWP), (i fnula (23), naNAWP),

- (fidata (24) (1:6), farNB5), (i fnula (24), nARNB5),

- (fidata (25) (1:6), faDAW5), (i fnula (25), naDAW5),

- (fidata (26) (1:20), farRC5), (i inula (26), nARRC5),

- (fidata (27) (1:1), faAsI5), (ifnula (27), nAASI5),

- (fidata (28) (1:1), fanATS), (i fnula (28), nanaTS),

- (fidata (29) (1:6), faRNB6), (i fnula (29), nARNB6),

- (fidata (30) (1:6), fadaw6), (ifnula (30), naDaw6),

- (fidata (31) (1:20), farrC6), (i fnula (31), naRRC6),

- (fidata (32) (1:1), faAsI6), (i fnula (32), nAASI6),

- (fidata (33) (1:1), faNaO), (i fnula (33), nANAO),

- (fidata (34) $(1: 6)$, fARNB7), (ifnula (34), nARNB7),

- (fidata (35) (1:6), fADAW7), (ifnula (35), nADAW7), 
- (fidatA (36) (1:20), fARRC7), (i fnula (36), nARRC7),

- (fidata (37) (1:1), fAASI7), (ifnula (37), nAASI7),

- (mxnoca (1), laSTI), (i fmoda (1), masTI),

- (mxnOcA (2), 1ACSN), (i $\operatorname{mmodA}(2), \operatorname{maCSN})$,

- (mXnOCA (3), IANAU), (i fmoda (3), mANAU),

- (mXnOcA (4), 1ARNB1), (ifmoda (4), maRnB1),

- (mxnoca (5), laDAw1), (ifmoda (5), madAW1),

- (mXnOCA (6), IARRC1), (i imoda (6), MARRC1),

- (mXnOCA (7), IAASI1), (ifmodA (7), mAASI1),

- (mxnoca (8), IANATh), (ifmoda (8), manaTh),

- (mxnoca (9), laRnB2), (ifmoda (9), maRnB2),

- (mxnOcA (10), IADAW2), (i fmoda (10), maDAW2),

- (mXnOcA (11), IARRC2), (i fmodA (11), maRRC2),

- (mXnoca (12), lAASI2), (i fmoda (12), maASI2),

- (mXnOCA (13), IANAPu), (i fmodA (13), mANAPu),

- (mXnOcA (14), 1ARNB3), (i imodA (14), mARNB3),

- (mXnOCA (15), IADAW3), (i fmoda (15), mADAW3),

- (mXnOCA (16), laRRC3), (i $\bmod A(16), \operatorname{maRRC3})$,

- (mXnOcA (17), lAASI3), (i $\operatorname{fmodA}(17), \operatorname{maASI3})$,

- (mXnOcA (18), IANASP), (i fmodA (18), mANASP),

- (mXnOCA (19), IARNB4), (i fmodA (19), maRNB4),

- (mXnOCA (20), IADAW4), (i imoda (20), mADAW4),

- (mXnOcA (21), laRRC4), (i fmoda (21), maRRC4),

- (mXnOCA (22), IAASI4), (i imodA (22), mAASI4),

- (mXnOcA (23), laNAWP), (i imoda (23), maNAWP),

- (mXnOcA (24), 1ARNB5), (i ifmoda (24), maRNB5),

- (mXnOCA (25), lADAW5), (i imoda (25), maDAW5),

- (mXnOcA (26), IARRC5), (i $\operatorname{mmodA}(26), \operatorname{maRRC5}$ ),

- (mxnoca (27), lAASI5), (i $\operatorname{fmoda}(27)$, maASI5),

- (mXnOCA (28), IANATS), (ifmodA (28), mANATS),

- (mXnOcA (29), IARNB6), (i imodA (29), maRNB6),

- (mXnOcA (30), lADAW6), (ifmoda (30), maDAW6),

- (mxnOcA (31), IARRC6), (i imoda (31), maRRC6),

- (mXnOcA (32), IAASI6), (i imoda (32), mAASI6),

- (mXnOCA (33), IANAO), (ifmodA (33), mANAO),

- (mxnOcA (34), IARNB7), (ifmodA (34), maRNB7),

- (mxnOcA (35), IADAW7), (i fmoda (35), mADAW7),

- (mXnOcA (36), IARRC7), (i imodA (36), maRRC7),

$\mathrm{C}$
$\mathrm{C}$
$\mathrm{C}$

- (mxnoca (37), LAASI7), (i imodA (37), mAASI7)

Database table and attribute names for screen fields.

data atrbta/

'ALPHA_URANIUM', 'SAMPLE TRACKING ID',

' SAMPLE_INFORMATION' , 'CŪSTOMER_SAMPLE_NAME',

' SAMPLE_INFORMATION' , NEED_ALPHA_URANIUM' ,

'ALPHA_URANIUM', 'RESULTS_NEEDED_BY_DATE' ,

'ALPHA_URANIUM', 'DATE_ALI_WORK_COMPLETED'

'ALPHA URANIUM', 'RESULTS REPORT CITATION',

'ALPHA_URANIUM' , 'ANY_SPECIAL_INSTRUCTIONS',

' SAMPLE_INFORMATION','NEED_ALPHA_THORIUM',

'ALPHA_THORIUM', 'RESULTS_NEEDED_BY_DATE',

'ALPHA_THORIUM', 'DATE_ALL_WORK_COMPLETED',

'ALPHA_THORIUM', 'RESULTS_REPORT_CITATION' ,

'ALPHA THORIUM', 'ANY_SPECIAL_INSTRUCTIONS',

' SAMPLE INFORMATION' ' NEED_ALPHA_PLUTONIUM',

'ALPHA_PLUTONIUM', 'RESULTS_NEEDED_BY_DATE' ,

'ALPHA_PLUTONIUM', 'DATE_ALI_WORK_COMPLETED',

'ALPHA_PLUTONIUM', 'RESULTS_REPORT_CITATION',

'ALPHA_PLUTONIUM', 'ANY_SPECIAL_INSTRUCTIONS', 
C

C

C

C

C

' SAMPLE_INFORMATION' ' NEED_ALPHA_AM241_SANS_PU238',

'AIPHA_AM241_SANS_PU238', 'RESULTS_NEEDED_BY_DATE' '

'ALPHA_AM241_SANS_PU238', 'DATE_ALL_WORK_COMPLETED' ,

'ALPHA_AM241_SANS_PU238', 'RESULTS_REPORT_CITATION' ,

'ALPHA_AM241_SANS_PU238', 'ANY_SPECIAL_INSTRUCTIONS' ,

' SAMPLE INFORMATION' ' NEED_ALPHA_AM2 41﹎WITH_PU238',

'AIPHA_AM241_WITH_PU238', 'RESULTS_NEEDED_BY_DATE' ,

'ALPHA_AM241_WITH_PU238', 'DATE_ALI_WORK_COMPLETED',

'ALPHA_AM241_WITH_PU238' ' 'RESULTS_REPORT_CITATION' ,

'ALPHA AM241_WITH_PU238' ' 'ANY_SPECIAL_INSTRUCTIONS'

'SAMPLE_INFORMATION' ' NEED_ALPHA_TOTAL_SPECTROMETRIC' ,

'ALPHA_TOTAL_SPECTROMETRIC', 'RESULTS_NEEDED_BY_DATE' '

'ALPHA TOTAL_SPECTROMETRIC', 'DATE_ALL_WORK_COMPLETED',

'ALPHA_TOTAL_SPECTROMETRIC', 'RESULTS_REPORT_CITATION' ,

'ALPHA_TOTAL_SPECTROMETRIC', 'ANY_SPECIAL_INSTRUCTIONS' ,

' SAMPLE_INFORMATION' , 'NEED_ALPHA_OTHER' ,

'ALPHA_OTHER' , 'RESULTS_NEEDED_BY_DATE' ,

'ALPHA_OTHER', 'DATE_ALL_WORK_COMPLETED' ,

'ALPHA_OTHER', 'RESULTS_REPORT_CITATION' '

- 'ALPHA_OTHER', 'ANY_SPECIAI_INSTRUCTIONS' /

Program variable names (value and null indicator) for screen fields.

data varblA/': fASTI : nASTI', : fACSN: nACSN' ' : fANAU: nANAU',

', iARNB1: nARNB1',' : IADAW1: ПADAW1', ': fARRC1: nARRC1',

- ' fAASI1:nAASI1' ' : fANATh: nANATh' , ' : iARNB2 : nARNB2',

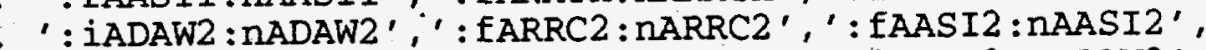

': fANAPu: IANAPu', ' : IARNB3 : nARNB3 ${ }^{\prime}, '$ ' : IADAW3 : nADAW3',

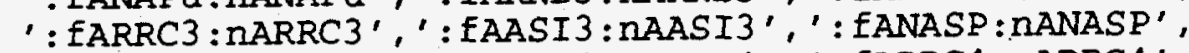

- ': iARNB4 : nARNB4 $', '$ : IADAW4 : nADAW4' ' : fARRC4 :nARRC4',

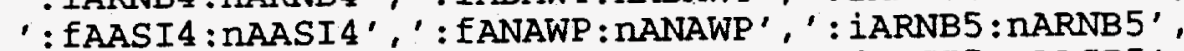

' ': iADAW5 : nADAW5',' : fARRC5: nARRC5',' : fAASI5: nAASI5',

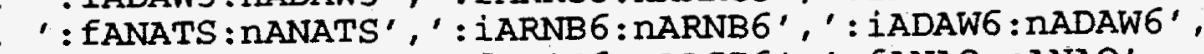

- ' : fARRC6:nARRC6', ' : fAASI6: nAASI6', ' : fANAO: nANAO',

. ' : iARNB7 : nARNB7', ' : IADAW7 : nADAW7', ' : fARRC7 : nARRC7',

. fAASI7:nAASI7',

Beta screen's field descriptors and field sizes.

character $* 80$ fieldB $(46)$, fidat $B(46)$

character $\star 30$ atrbtB $(2,46)$

character 14 varb1B (46)

character*1 fitypB $(46)$

integer*4 falenB $(46), f \operatorname{drowB}(46), f d c o l B(46), f i c o l B(46), \operatorname{mxnocB}(46)$,

. fdrndB $(3,46)$, firndB $(3,46)$, ! Field renditions (add/update/search).

- numfdB/46/ ! The number of fields.

integer $* 2$ ifnulB $(46)$,

ifrgdB $(46) / 46 * 0 /$

iditoB $(46) / 46 * 0 /$

logical ifmodB (46)

!Null indicator $(-1$ if null, 0 otherwise). ! Has a field been modified in an Update? common/dbdat $B / f i e l d B$, fidat $B$, fdlenB, fdrowB, fdcolB, ficolB, mxnocB, fdrndB, firndB, numfdB, fitypB, ifnulB, atrbt $B$, varblB, ifrqdB, ifmodB,

- iditoB

Length-specified character fields for equivalences (f for field).

character $\mathrm{fBSTI} \star 12, \mathrm{fBCSN} * 60, \mathrm{fBNBSr} * 1, \mathrm{fBDCL} 1 \star 4, \mathrm{fBDCU} 1 * 1, \mathrm{fBRND} 1$ * 6 , fBRNT $1 * 5, \mathrm{fBDAW} 1 * 6, \mathrm{fBRRC} 1 * 20, \mathrm{fBASI} 1 * 1, \mathrm{fBNBSC} * 1, \mathrm{fBDCL} 2 * 4, \mathrm{fBDCU} 2 * 1$, - fBRNT $1 * 6, \mathrm{fBRNT} 2 * 5, \mathrm{fBDAW} 2 * 6, \mathrm{fBRRC} 2 * 20, \mathrm{fBASI} 2 * 1, \mathrm{fBNBST} * 1, \mathrm{fBDCL} 3 * 4$, - fBDCU $3 * 1$, fBRND $3 * 6$, fBRNT 3 * 5, fBDAW $3 * 6$, fBRRC $3 * 20, f B A S I 3 * 1, f B N B T * 1$, 
- $\mathrm{fBDCL} 4$ * $4, \mathrm{fBDCU} 4$ * $1, \mathrm{fBRND} 4$ *6, fBRNT4 *5, fBDAW4 *6, fBRRC4 *20,fBASI 4 * 1 ,

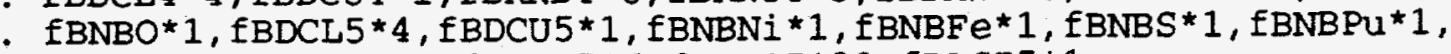

- fBRND5*6, fBRNT5*5, £BDAW5*6, fBRRC5*20, fBASI5*1

Real and integer field storage ( $r$ for real, $i$ for integer).

rea 1 * 4 rBDCL1, rBDCL2 $, r B D C L 3, r B D C L 4, r B D C L 5$

integer * 4 iBRND1, iBDAW1, iBRND2 , iBDAW2, iBRND3, iBDAW3 , iBRND4, iBDAW4, - IBRND5, IBDAW5

\section{Nonarrayed maximum field lengths ( 1 for length).}

integer*4 1BSTI, 1BCSN, 1BNBSr, 1BDCL1, 1BDCU1, 1BRND1, 1BRNT1, 1BDAW1, IBRRC1, IBASI1, IBNBSC, 1 BDCL2, 1BDCU2, IBRND2, 1BRNT2, 1 BDAW2, 1 BRRC2,

- IBASI2, 1BNBST, 1BDCL 3, 1BDCU3, 1BRND3, 1BRNT3, 1BDAW3, 1BRRC3, 1BASI3, IBNBT, IBDCL4, IBDCU4, IBRND4, IBRNT4, IBDAW4, 1BRRC4, IBASI4, 1 BNBO,

- 1BDCL5, 1 BDCU5, 1BNBNi , IBNBFe, IBNBS, 1BNBPU, IBRND5, IBRNT5, 1 BDAW5,

- IBRRC5, 1BASI5

\section{Nonarrayed null indicators for data transfers (n for null).}

integer *2 nBSTI, nBCSN, nBNBSr, nBDCL1, nBDCU1, nBRND1, nBRNT1, nBDAW1, - nBRRC1, nBASI1, nBNBSC, nBDCL2, nBDCU2, nBRND2, nBRNT2, nBDAW2, nBRRC2, - nBASI2, nBNBST, nBDCL3, nBDCU3, nBRND3, nBRNT3, nBDAW3, nBRRC3, nBASI3, nBNBT, nBDCL 4, nBDCU4 , nBRND4 , nBRNT4 , nBDAW4, nBRRC4, nBASI4 , nBNBO,

- nBDCL5, nBDCU5, nBNBNi , nBNBFe, nBNBS, nBNBPu, nBRND5, nBRNT5, nBDAW5,

- nBRRC5, nBASI5

Nonarrayed if-modified indicators (m for modified).

integer *2 mBSTI, MBCSN, mBNBSr, MBDCL1, mBDCU1, MBRND1, MBRNT1, MBDAW1, MBRRC1, MBASI1, MBNBSC, MBDCL2, MBDCU2, MBRND2, MBRNT2, mBDAW2, MBRRC2,

- mBASI2, mBNBST, mBDCL3, mBDCU3, mBRND3, mBRNT3, mBDAW3, mBRRC 3, mBASI3, mBNBT, mBDCL 4 , mBDCU4 , mBRND4 , mBRNT4 , MBDAW4 , MBRRC4 , MBASI4 , MBNBO, - mBDCL 5 , mBDCU 5, mBNBNi, mBNBFe, mBNBS, mBNBPu, mBRND5, mBRNT5, mBDAW5, - mBRRC5, MBASI5

C

C

Equivalence statements for data transfers.

equivalence (fidatB (1) (1:12), fBSTI), (ifnulB (1), nBSTI),

- (fidatB $(2)(1: 60), \mathrm{fBCSN}),($ i fnulB $(2), \mathrm{nBCSN})$,

- (fidatB (3) $(1: 1)$, fBNBSr), (ifnulB (3), nBNBSr),

- (fidat $(4)(1: 4)$, fBDCL1), (i fnulB $(4)$, nBDCL 1$)$,

- (fidatB (5) (1:1), fBDCU1), (i fnulB (5), nBDCU1).

- (fidatB $(6)(1: 6)$, fBRND1), (ifnulB $(6)$, nBRND1).

- (fidat $(7)(1: 5)$, fBRNT1), (i fnulB (7), nBRNT1),

- (fidatB (8) $(1: 6)$, fBDAW 1$)$, (i fnulB $(8)$, nBDAW 1 ),

- (fidatb (9) $(1: 20)$, fBRRC1), (ifnulb (9), nBRRC1),

- (fidatB (10) (1:1), fBASI1), (ifnulb (10), nBASI1),

$\therefore$ (fidatB (11) (1:1), fBNBSC), (ifnulB (11), nBNBSC),

- (fidatB (12) (1:4), fBDCL2), (ifnulB (12), nBDCL2),

- (fidatB (13) (1:1), fBDCU2), (ifnulB (13), nBDCU2),

- (fidatB (14) $(1: 6)$, fBRND2), (ifnulB (14), nBRND2),

- (fidatB (15) $(1: 5)$, fBRNT2), (i fnulB (15), nBRNT2),

- (fidatB (16) (1:6), fBDAW2), (ifnulb (16), nBDAW2),

- (fidatB (17) (1:20), EBRRC2), (i fnulB (17), nBRRC2),

- (fidatB (18) (1:1), fBASI2), (ifnulb(18), nBASI2),

- (fidatB (19) $(1: 1)$, fBNBST), (ifnulB $(19)$, nBNBST),

- (fidatB $(20)(1: 4)$, fBDCL3), (ifnulB $(20), n B D C L 3)$,

- (fidatB $(21)(1: 1)$, fBDCU3), (ifnulB (21), nBDCU3), 
- (fidatB $(22)(1: 6), f B R N D 3)$, (ifnulB (22), nBRND3), (fidatB (23) (1:5), fBRNT3), (ifnulB (23), nBRNT3),

- (fidatB (24) (1:6), fBDAW3), (ifnulB (24), nBDAW3), (fidatB (25) (1:20), fBRRC3), (i fnulb (25), nBRRC3),

- (fidatB $(26)(1: 1)$, fBASI3), (ifnulB (26), nBASI3),

- (fidatB (27) (1:1), fBNBT), (ifnulB (27), nBNBT),

- (fidatB $(28)(1: 4)$, fBDCL4), (ifnulB (28), nBDCL4), (fidatB (29) (1:1), fBDCU4), (i fnulB (29), nBDCU4),

- (fidatB $(30)(1: 6)$, fBRND4), (i fnulB (30), nBRND4),

- (fidatB (31) (1:5), fBRNT4), (ifnulB (31), nBRNT4),

(fidatB (32) ( $1: 6$ ), fBDAW4), (ifnulB (32), nBDAW4),

(fidatB (33) (1:20), fBRRC4), (ifnulB (33) inBRRC4),

- (fidatB (34) (1:1), fBASI4), (ifnulB (34), nBASI4),

- (fidatB (35) (1:1), fBNBO), (ifnulB (35), nBNBO),

- (fidatB $(36)(1: 4)$, fBDCL5), (ifnulB (36), nBDCL5),

- (fidatB (37) (1:1), fBDCU5), (ifnulB (37), nBDCU5),

- (fidatB $(38)(1: 1)$, fBNBNi), (ifnulB (38), nBNBNi),

- (fidatB $(39)(1: 1)$, fBNBFe), (ifnulB (39), nBNBFe),

- (fidatB (40) (1:1), fBNBS), (ifnulB (40), nBNBS),

- (fidatB $(41)(1: 1)$, IBNBPu), (ifnulB $(41), n B N B P u)$,

- (fidatB $(42)(1: 6)$, fBRND5), (i inulB (42), nBRND5),

- (fidatB (43) ( $1: 5$ ), fBRNT5), (ifnulB (43), nBRNT5),

- (fidatB $(44)(1: 6)$, fBDAW5), (i fnulB (44), nBDAW5),

- (fidatB (45) (1:20), fBRRC5), (ifnulB (45), nBRRC5),

- (fidatB (46) (1:1), fBASI5), (ifnulB (46), nBASI5),

- (mxnocB (1), IBSTI), (ifmodB (1), mBSTI),

- (mxinocB $(2), I B C S N)$, (ifmodB (2), mBCSN),

- (mxnocB (3), lBNBSr), (ifmodB (3), mBNBSr),

- (mxnocB $(4)$, lBDCL1), (i $\operatorname{limodB}(4), \operatorname{mBDCL} 1)$,

- (mxnocB (5), IBDCU1), (ifmodB (5), mBDCU1),

- (mXnOCB $(6)$, IBRND1), (ifmodB $(6)$, mBRND1),

- (mxnOcB (7), IBRNT1), (ifmodB (7), mBRNT1),

- (mXnOCB (8), IBDAW1)), (ifmodB (8), mBDAW1),

- (mxnOCB (9), IBRRC1), (ifmodB (9), mBRRC1),

- (mxnocB (10), IBASI1), (i $\operatorname{modB}(10), \operatorname{mBASI} 1)$,

- (mxnocB (11), IBNBSC), (ifmodB (11), mBNBSC),

- (mXnOCB (12), IBDCL2), ( i $\operatorname{modB}(12), \operatorname{mBDCL} 2)$,

- (mxnOCB (13), 1BDCU2), ( i fmodB (13), mBDCU2),

- (mXnOCB (14), 1BRND2), (ifmodB (14), mBRND2).

- (mXnOcB (15), IBRNT2), (ifmodB (15), mBRNT2),

- (mxnocB (16), IBDAW2), (i $\operatorname{modB}(16)$, mBDAW2),

- (mXnOcB (17), IBRRC2), (ifmodB (17), mBRRC2),

- (mXnOcB (18), IBASI2)', (i fmodB (18), mBASI2),

- (mXnOCB (19), IBNBST), (i imodB (19), mBNBST),

- (mXnOcB $(20), 1 B D C L 3)$, (i imodB $(20)$, mBDCL3),

- (mxnOcB (21), IBDCU3), (ifmodB (21), mBDCU3),

- (mXnOCB (22), IBRND3), (ifmodB (22), mBRND3),

- (mXnocB (23), IBRNT3), (ifmodB (23), mBRNT3),

- (mXnOcB (24), IBDAW3), (i $\operatorname{modB}(24), \operatorname{mBDAW} 3)$,

- (mXnOcB (25), 1BRRC3), (i fmodB (25), mBRRC3),

- (mxnocb $(26), 1$ BASI3), (i fmodB (26), mBASI3),

- (mxnOcB $(27), 1$ BNBT), (ifmodB (27), mBNBT),

- (mXnOcB $(28), 1$ BDCL4), (i $\operatorname{modB}(28), \operatorname{mBDCL} 4)$,

- (mxnocB (29), IBDCU4), (i $\operatorname{modB}(29)$, mBDCU4),

- (mXnOcB (30), 1BRND4), (i imodB (30), mBRND4),

- (mXnOCB (31), IBRNT4), (i $\operatorname{mmodB}(31)$, mBRNT4),

- (mXnOcB (32), IBDAW4), (i fmodB (32), mBDAW4),

- (mXnocB (33), I BRRC4), (i fmodB (33), mBRRC4),

- (mXnocb (34), IBASI4), (i $\operatorname{modB}(34), \operatorname{mBASI} 4)$,

- (mxnocB (35), 1BNBO), (ifmodB (35), mBNBO), 
- (mxnocB (36), 1BDCL5), (ifmodB (36), mBDCL5), - (mxnOCB (37), IBDCU5), (i imodB (37), mBDCU5), - (mXnOCB (38), IBNBNi), (i fmodB (38), mBNBNi), - (mxnocB $(39), 1 B N B F e)$, (i fmodB (39), mBNBFe), - (mxnocB $(40), 1 B N B S)$, (ifmodB (40), mBNBS),

- (mxnOcB (41), 1BNBPu), (i $\operatorname{modB}(41), \operatorname{mBNBPu})$, - (mXnOCB (42), 1BRND5), (i $\operatorname{modB}(42), \operatorname{mBRND5}$ ), - (mXnOcB (43), IBRNT5), (i $\operatorname{modB}(43)$, mBRNT5), - (mXnOcB (44), IBDAW5), (i fmodB (44), mBDAW5).

- (mXnOcB (45), 1BRRC5), (ifmodB (45), mBRRC5),

- (mxnOcB (46), 1BASI5), (ifmodB (46), mBASI5)

Database table and attribute names for screen fields.

data atrbtB/

'BETA_STRONTIUM_90', 'SAMPLE_TRACKING_ID',

' SAMPLE_INFORMATION' , CUSTOMER_SAMPLE_NAME',

'SAMPLE_INFORMATION', 'NEED_BETA_STRONTIUM_90',

'BETA_STRONTIUM_90', 'DETECTOR_COUNT_LENGTH',

'BETA_STRONTIUM_90', 'DETECTOR_COUNT_LENGTH_UNITS',

'BETA_STRONTIUM_90', 'RESULTS_NEEDED_BY_DATE',

'BETA_STRONTIUM_90','RESULTS_NEEDED_BY_TIME',

'BETA_STRONTIUM_90', 'DATE_ALL_WORK_COMPLETED',

'BETA_STRONTIUM_90','RESULTS_REPORT_CITATION',

'BETA_STRONTIUM_90', 'ANY_SPECIAL_INSTRUCTIONS'

'SAMPLE_INFORMATION' , NEED_BETA_STRONTIUM_89_AND_90',

'BETA_STRONTIUM_89_AND_90', 'DETECTOR_COUNT_LENGTH',

'BETA_STRONTIUM_89_AND_90','DETECTOR_COUNT_LENGTH_UNITS',

'BETA_STRONTIUM_89_AND_90', 'RESULTS_NEEDED_BY_DATE',

'BETA_STRONTIUM_89_AND_90', 'RESULTS_NEEDED_BY_TIME',

'BETA_STRONTIUM_89_AND_90', 'DATE_ALL_WORK_COMPLETED',

'BETA_STRONTIUM_89_AND_90', 'RESULTS_REPORT_CITATION' ,

'BETA_STRONTIUM_89_AND_90', 'ANY_SPECIAL_INSTRUCTIONS',

'SAMPLE_INFORMATION', 'NEED_BETA_TOTAL_STRONTIUM',

'BETA_TOTAL_STRONTIUM', 'DETECTOR_COUNT_LENGTH',

'BETA_TOTAL_STRONTIUM', 'DETECTOR_COUNT_LENGTH_UNITS' ,

'BETA_TOTAL_STRONTIUM', ' RESULTS_NEEDED_BY_DATE',

'BETA_TOTAL_STRONTIUM', 'RESULTS_NEEDED_BY_TIME',

'BETA_TOTAL_STRONTIUM',' DATE_ALI_WORK_COMPLETED',

'BETA_TOTAI_STRONTIUM', 'RESULTS_REPORT_CITATION',

'BETA_TOTAL_STRONTIUM', 'ANY_SPECIAL_INSTRUCTIONS',

' SAMPLE_INFORMATION', 'NEED_BETA_TRITIUM',

'BETA_TRITIUM', 'DETECTOR_COUNT_LENGTH',

'BETA_TRITIUM', 'DETECTOR_COUNT_LENGTH_UNITS',

'BETA_TRITIUM' , 'RESULTS_NEEDED_BY_DATE',

'BETA_TRITIUM','RESULTS_NEEDED_BY_TIME',

'BETA_TRITIUM', 'DATE_ALL__WORK_COMPLETED' ,

'BETA_TRITIUM',' RESULTS_REPORT_CITATION',

'BETA_TRITIUM', 'ANY_SPECIAL_INSTRUCTIONS',

' SAMPLE_INFORMÁTION',' NEED_BETA_OTHER',

'BETA_OTHER', 'DETECTOR_COUNT_LENGTH',

'BETA_OTHER', 'DETECTOR_COUNT_LENGTH_UNITS',

'BETA_OTHER', 'NEED_BETA_NICKEL_63',

'BETA_OTHER', 'NEED_BETA_IRON_55',

'BETA_OTHER', 'NEED_BETA_SULFUR_35',

'BETA_OTHER', 'NEED_BETA_PLUTONIUM_241',

'BETA_OTHER', 'RESUITS_NEEDED_BY_DATE',

'BETA_OTHER', 'RESULTS_NEEDED_BY_TIME',

'BETA_OTHER', 'DATE_ALI_WORK_COMPLETED',

'BETA_OTHER', 'RESULTS_REPORT_CITATION' , 
C
C

C
C
C

C
C
C

C
C
C

C
C
C

C

. 'BETA_OTHER' ,'ANY_SPECIAL_INSTRUCTIONS'/

Program variable names (value and null indicator) for screen fields.

data varblB /' :fBSTI:nBSTI',' : fBCSN:nBCSN' ,' : fBNBSr:nBNBSr',

' : rBDCL1 : nBDCL1',' : fBDCU1 : nBDCU1',': iBRND1 :nBRND1',

' $:$ fBRNT1 : nBRNT1', ' : iBDAW1 : nBDAW1', ' : fBRRC1 : nBRRC1',

' : fBASI1 : nBASI1', ' : fBNBSC : nBNBSC', ' : rBDCL2 : nBDCL2',

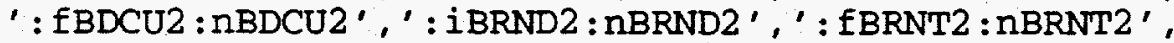

' : iBDAW2 : nBDAW2', ' : fBRRC2 : nBRRC2', ' : fBASI2 :nBASI2',

$'$ : fBNBST : nBNBST', ' : rBDCL 3 : nBDCL3', ' : fBDCU3 : nBDCU3',

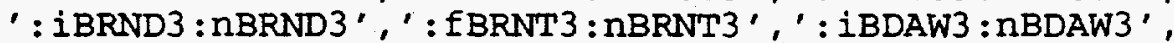

' : fBRRC3 :nBRRC 3',' : fBASI 3 :nBASI3', ' : fBNBT: nBNBT',

' rBDCL4 : nBDCL4 ', ' : fBDCU4 :nBDCU4 ', ' : iBRND4 : nBRND4',

' : fBRNT4 : nBRNT4', ' : i BDAW4 : nBDAW4', ' : fBRRC4 :nBRRC4',

' : fBASI4 :nBASI4 ' ', : fBNBO:nBNBO' , : rBDCL5 : nBDCL 5',

$'$ : fBDCU5 : nBDCU5', ' : fBNBNi :nBNBNi', ' : fBNBFe:nBNBFe',

' : fBNBS : nBNBS', ' : IBNBPu : nBNBPu', ' i iBRND5 : nBRND5',

' : fBRNT5 :nBRNT5', ' : iBDAW5 : nBDAW5', ': fBRRC5:nBRRC5',

' fBASI5:nBASI5' $/$

Gamma screen's field descriptors and field sizes.

character $* 80$ fieldG (35), fidatG (35)

character $* 30$ atrbtg $(2,35)$

character ${ }^{*} 14$ varblg (35)

character 1 fitypg (35)

integer 4 fdleng (35), fdrowg (35), fdcolg (35), ficolg (35), mxnocG (35),

. fdrndG (3,35), firndG $(3,35)$, !Field renditions (add/update/search).

- numfdG/35/ !The number of fields.

integer 2 ifnulg(35), ! Null indicator $(-1$ if null, 0 otherwise).

- ifrgdG (35) $135 * 0 \%$, Points to if-required dependent field.

- iditoG(35)/35*0/ ! Points to allowed field for dittoing.

logical ifmodg(35) ! Has a field been modified in an Update?

common /dbdatG/ fieldG, fidatG, fdleng, fdrowg, fdcolG, ficolg, mxnocG,

- fdrndG, firndG, numfdG, fitypg, ifnulG, atrbtG, varblG, ifrqdG, ifmodG,

- iditog

Length-specified character fields for equivalences ( $f$ for field).

character fGSTI * 12, fGCSN*60, fGNGS 11, fGDCL 1 * 4, fGDCU1 1 1, fGRND 1 * 6 ,

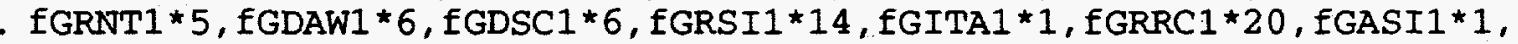

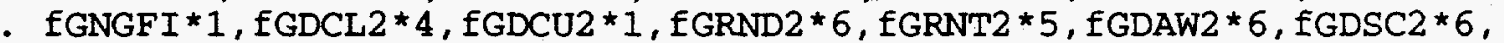

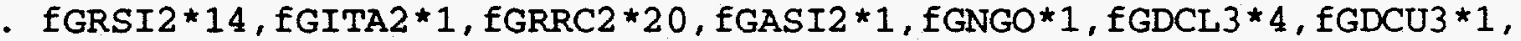

. fGRND $3 * 6, \mathrm{fGRNT} 3 * 5, \mathrm{fGDAW} 3 * 6, \mathrm{fGDSC} 3 * 6, \mathrm{fGRSI} 3 * 14, \mathrm{fGITA} 3 * 1, \mathrm{fGRRC} 3 * 20$,

- fGASI $3 \star 1$

Real and integer field storage (r for real, $i$ for integer).

real 4 rGDCL1, rGDCL2, rGDCL3

integer $* 4$ iGRND1, iGDAW1, iGDSC1; iGRND2 , iGDAW2 , iGDSC2 , iGRND3 , iGDAW3 , . iGDSC3

Nonarrayed maximum field lengths ( 1 for length).

integer *4 1GSTI, 1GCSN, 1GNGS, 1GDCL1, 1GDCU1, 1GRND1, 1GRNT1, 1GDAW1, - 1GDSC1, 1GRSI1, 1GITA1, 1GRRC1, 1GASI1, 1GNGFI, 1GDCL2 , 1GDCU2, 1GRND2,

- 1GRNT2, 1GDAW2, 1GDSC2, 1GRSI2, 1GITA2 , 1GRRC2, 1GASI2 , 1GNGO, 1GDCL 3 ,

- 1GDCU3, 1GRND3, 1GRNT3, 1GDAW3, 1GDSC3, 1GRSI3, 1GITA3, 1GRRC3, 1GASI3 
integer $2^{2}$ nGSTI, nGCSN, nGNGS, nGDCL1, nGDCU1, nGRND1, nGRNT1, nGDAW1, - nGDSC1, nGRSI1, nGITA1, nGRRC1, nGASI1, nGNGFI, nGDCL2, nGDCU2 , nGRND2, - nGRNT2, nGDAW2, nGDSC2, nGRSI2, nGITA2, nGRRC2, nGASI2, nGNGO, nGDCL3, - nGDCU3, nGRND3, nGRNT3, nGDAW3, nGDSC3, nGRSI3, nGITA3, nGRRC3, nGASI3

$\mathrm{C}$

$\mathrm{C}$

C

$\mathrm{C}$

C

$\mathrm{C}$

$$
\text { Nonarrayed if-modified indicators (m for modified). }
$$

integer ${ }^{2}$ mGSTI , mGCSN , MGNGS, mGDCL1, mGDCU1 , mGRND1, MGRNT1, MGDAW1, MGDSC1, MGRSI1, MGITA1, MGRRC1, MGASI1, MGNGFI , MGDCL2 , mGDCU2 , mGRND2 ,

- MGRNT2 , mGDAW2, mGDSC2, mGRSI2, mGITA2 , mGRRC2, MGASI2, mGNGO, MGDCL 3 ,

- mGDCU3, mGRND3, mGRNT3, mGDAW3, mGDSC 3, mGRSI 3, mGITA3, mGRRC3, mGASI3

\section{Equivalence statements for data'transfers.}

equivalence (fidatg(1) (1:12), fGSTI), (ifnulG(1), nGSTI),

- (fidatG (2) $(1: 60)$, fGCSN), (ifnulG (2), nGCSN),

- (fidatg (3) $(1: 1)$, fGNGS), (ifnulG (3), nGNGS),

- (fidatG (4) (1:4), fGDCL1), (ifnulg(4), nGDCL1),

- (fidatG(5) (1:1), fGDCU1), (ifnulg(5), nGDCU1),

- (fidatG (6) (1:6), EGRND1), (ifnulG (6), nGRND1),

- (fidatG (7) (1:5), fGRNT1), (ifnulg (7), nGRNT1),

- (fidatg (8) (1:6), fGDAW1), (i fnulg (8), nGDAW1),

- (fidatg $(9)(1: 6)$, fGDSC1), (ifnulg (9), nGDSC1),

- (fidatg(10) (1:14), fGRSI1), (i fnulg(10), nGRSI1),

- (fidatg(11) (1:1), EGITA1), (ifnulg(11), nGITA1),

- (fidatg (12) (1:20), fGRRC1), (i fnulG(12), nGRRC1),

- (fidatg(13)(1:1), fGASI1), (ifnulg(13), nGASI1),

- (fidatg (14) (1:1), fGNGFI), (ifnulg (14), nGNGFI),

- (fidatG (15) (1:4), fGDCL2). (ifnulg (15), nGDCL2),

- (fidatg (16), (1:1), fGDCU2), (ifnulg (16), nGDCU2),

- (fidatG(17) $(1: 6)$, fGRND2), (ifnulg (17), nGRND2),

- (fidatg (18) (1:5), EGRNT2), (ifnulg (18), nGRNT2),

- (fidatg (19) (1:6), fGDAW2), (ifnulg(19), nGDAW2).

- (fidatg(20) (1:6), fGDSC2), (ifnulg (20), nGDSC2),

- (fidatg (21) (1:14), fGRSI2), (i fnulg(21), nGRSI2),

$\because$ (fidatg(22) (1:1), fGITA2), (ifnulg(22), nGITA2),

- (fidatg(23) $(1: 20)$, fGRRC2), (i fnulg(23), nGRRC2),

- (fidatg (24) (1:1), fGASI2), (ifnulg (24), nGASI2),

- (fidatG (25) (1:1), fGNGO), (i fnulg (25), nGNGO),

- (fidatg $(26)(1: 4)$, fGDCL 3$)$, (ifnulg (26), nGDCL 3$)$,

- (fidatg (27) (1:1), fGDCU3), (ifnulg (27), nGDCU3),

- (fidatg (28) (1:6), fGRND3), (ifnulg (28), nGRND3),

- (fidatg(29) (1:5), fGRNT3), (ifnulg (29), nGRNT3),

- (Eidatg (30) (1:6), fGDAW3), (ifnulg (30), nGDAW3),

- (fidatG(31) $(1: 6)$, fGDSC 3$)$, (ifnulg (31), nGDSC3),

- (fidatg (32) (1:14), fGRSI3), (i fnulg(32), nGRSI3),

- (fidatg(33) (1:1), fGITA3), (ifnulg (33), nGITA3),

- (fidatg (34) (1:20), fGRRC3), (i fnulg (34), nGRRC3),

- (fidatg(35) (1:1), fGASI3), (ifnulg(35), nGASI3),

- (mxnocG (1), IGSTI), (i fmodG (1), mGSTI),

- (mxnocG $(2), 1 G C S N)$, (i $\operatorname{modg}(2), \operatorname{mGCSN})$,

- (mxnocG (3), lGNGS), (i fmodG (3), mGNGS),

- (mXnocG (4), 1GDCL1), (ifmodG (4), mGDCL1),

$\therefore$ (mXnOcG (5), 1 GDCU1), (ifmodG (5), mGDCU1),

- (mXnocG $(6)$, l GRND1), (i $\bmod$ ( 6 ), MGRND1),

- (mXnOcG (7), I GRNT1), (i fmodG (7), mGRNT1),

- (mXnocG (8), I GDAW1), (i imodG (8), mGDAW1),

- (mxnocG (9), IGDSC1), (ifmodG (9), mGDSC1), 
. (mxnocG (10), 1GRSI1), (ifmodG (10), mGRSI1),

- (mxnocG (11), IGITA1), (i fmodG (11), mGITA1),

- (mXnocG (12), 1GRRC1), (i fmodG (12), mGRRC1),

- (mxnocG (13), IGASI1), (ifmodG (13), mGASI1),

- (mxnocG (14), 1GNGFI), (ifmodG (14), mGNGFI),

- (mxnocG (15), 1GDCL2), (i fmodG (15), mGDCL2),

- (mxnocG (16), IGDCU2), (i $\operatorname{fmodg}(16), \operatorname{mGDCU} 2)$,

- (mXnocG (17), 1GRND2), (i imodG (17), mGRND2),

- (mxnOcG (18), 1GRNT2), (ifmodG (18) , mGRNT2),

- (mXnocG (19), IGDAW2), ( i fmodG (19), mGDAW2),

- (mxnocG $(20), 1$ GDSC2), (i $\operatorname{mmodg}(20), \operatorname{mGDSC} 2)$,

- (mxnocG (21), 1GRSI2), (i fmodG (21), mGRSI2),

- (mxnocG (22), IGITA2), (i fmodG (22), mGITA2),

- (mXnocG (23), 1GRRC2), (i fmodG (23), mGRRC2),

- (mxnocG (24), 1GASI2), (i fmodG (24), mGASI2),

- (mxnocG (25), 1GNGO), (ifmodG (25), mGNGO).

- (mXnocG $(26), 1$ GDCL 3) , (ifmodg (26), mGDCL3) ,

- (mxnocG (27), 1GDCU3), (i fmodG (27), mGDCU3),

- (mXnocG (28), 1GRND3), (i fmodG (28), mGRND3),

- (mXnOcG (29), 1GRNT3), (i fmodG (29), mGRNT3),

- (mXnocG $(30), 1$ GDAW3), (ifmodG (30), mGDAW3),

- (mxnocG (31), lGDSC3), (ifmodG (31), mGDSC3),

- (mxnocG (32), lGRSI3), (i fmodG (32), mGRSI3),

- (mxnocG (33), IGITA3), (i fmodG (33), mGITA3),

- (mxnocG (34), 1GRRC3), (i fmodg (34), mGRRC3),

- (mxnocG (35), lGASI3), (ifmodG (35), mGASI3)

$\mathrm{C}$
$\mathrm{C}$
$\mathrm{C}$

Database table and attribute names for screen fields.

data atrbtG/

'GAMMA_SCREEN', 'SAMPLE_TRACKING_ID',

'SAMPLE_INFORMATION', 'CUSTOMER_SAMPLE_NAME',

' SAMPLE_INFORMATION' , 'NEED_GAMMA_SCREEN',

' GAMMA_SCREEN', 'DETECTOR_COUNT_LENGTH',

'GAMMA_SCREEN',' DETECTOR_COUNT_LENGTH_UNITS',

'GAMMA_SCREEN' , 'RESULTS_NEEDED_BY_DATE',

'GAMMA_SCREEN' , 'RESULTS_NEEDED_BY_TIME',

'GAMMA_SCREEN', 'DATE_ALE__WORK_COMPLETED',

' GAMMA_SCREEN' , 'DATE_SAMPLE_COUNTED' ,

'GAMMA_SCREEN','RML_SPECTRAL_ID',

'GAMMA_SCREEN', 'IS_THIS_A_SPECTRUM_RECOUNT',

'GAMMA_SCREEN' 'RESULTS_REPORT_CITATION',

' GAMMA_SCREEN' ' ANY_SPECIAL_INSTRUCTIONS',

'SAMPIE_INFORMATION' ' NEED_GAMMA_FULL_ISOTOPIC' ,

'GAMMA_FULI_ISOTOPIC', 'DETECTOR_COUNT_LENGTH',

'GAMMA_FULI_ISOTOPIC', 'DETECTOR_COUNT_LENGTH_UNITS',

'GAMMA_FULI_ISOTOPIC', 'RESULTS_NEEDED_BY_DATE',

'GAMMA_FULI_ISOTOPIC', 'RESULTS_NEEDED_BY_TIME',

'GAMMA_FULI_ISOTOPIC', ' DATE_ALE_WORK_COMPLETED',

'GAMMA_FULI_ISOTOPIC', 'DATE_SAMPLE_COUNTED',

'GAMMA_FULL_ISOTOPIC',' RML_SPECTRAL_ID',

' GAMMA_FULI_ISOTOPIC', 'IS_THIS_A_SPECTRUM_RECOUNT',

'GAMMA_FULI_ISOTOPIC', 'RESULTS_REPORT_CITATION',

'GAMMA_FULL_ISOTOPIC', ' ANY_SPECIAL_INSTRUCTIONS',

'SAMPIE__INFORMATION', 'NEED_GAMMA_OTHER',

' GAMMA_OTHER' , DETECTOR_COUNT_LENGTH' ,

'GAMMA_OTHER', 'DETECTOR_COUNT_LENGTH_UNITS',

' GAMMA_OTHER', 'RESULTS_NEEDED_BY_DATE',

'GAMMA_OTHER', 'RESULTS_NEEDED_BY_TIME',

'GAMMA_OTHER'.' DATE_ALI_WORK_COMPLETED' , 
. 'GAMMA_OTHER', 'DATE_SAMPLE_COUNTED',

'GAMMA_OTHER', 'RMI_SPECTRAL_ID',

'GAMMA_OTHER', 'IS_THIS_A_SPECTRUM_RECOUNT',

' 'GAMMA_OTHER', 'RESULTS_REPORT_CITATION',

- 'GAMMA_OTHER', 'ANY_SPECIAI_INSTRUCTIONS' /

$\mathrm{C}$

C

$\mathrm{C}$

C

C

C

$\mathrm{C}$

C

$\mathrm{C}$

C

C

C

C

C

$\mathrm{C}$

C

Program variable names (value and null indicator) for screen fields.

data varblG/': fGSTI:nGSTI', ': fGCSN: nGCSN' ,':fGNGS :nGNGS',

. ' : rGDCL1 :nGDCL1',' : fGDCU1 : nGDCU1',': iGRND1 :nGRND1',

. ' : fGRNT1 : nGRNT1', ' : iGDAW1 : nGDAW1', ': iGDSC1 : nGDSC1',

. ' : fGRSI1:nGRSI1', ' : fGITA1 : nGITA1', ': fGRRC1 :nGRRC1',

.' fGASI1 : nGASI1', ' fGNGFI : nGNGFI', ' : rGDCL2 : nGDCL2',

' : fGDCU2 : nGDCU2 ', ' : iGRND2 : nGRND2', ' : fGRNT2 : nGRNT2',

': iGDAW2 : nGDAW2', ' : iGDSC2 :nGDSC2' ' : fGRSI2 : nGRSI2',

' : fGITA2 : nGITA2', ' : fGRRC2 : nGRRC2', ' : fGASI2 : nGASI2',

': fGNGO: nGNGO', : rGDCL 3 : nGDCL3' ,' : fGDCU3 : nGDCU3',

' : iGRND3 : nGRND3', ' : fGRNT3 : nGRNT3' , ' : iGDAW3 : nGDAW3',

': iGDSC 3 : nGDSC 31, ' : fGRSI3 : nGRSI3', ': fGITA3 :nGITA3',

' ' : fGRRC3 : nGRRC3', ' : fGASI3 : nGASI3'/

Gross alpha-beta screen's field descriptors and field sizes.

character $* 80$ fieldC (18), fidatc (18)

character $* 30$ atrbtc $(2,18)$

character $* 14$ varblc (18)

character*1 fitypc (18)

integer 4 fdlenc (18), fdrowC (18), fdcolc (18), ficolc (18), mxnocc (18),

- fdrndC $(3,18)$, firndC $(3,18)$, !Field renditions (add/update/search).

- numfac/18/ !The number of fields.

integer*2 ifnulc(18), !Null indicator (-1 if null, 0 otherwise).

- ifrqdC (18)/18*0\%, ! points to if-required dependent field.

- iditoc(18)/18*0/ ! Points to allowed field for dittoing.

logical ifmodC(18) ! Has a field been modified in an Update?

common /dbdatc/ fieldC, fidatc, fdlenc, fdrowC, fdcolc, ficolc, mxnocC,

- fdrndC, firndC, numfdC, fitypc, ifnulc, atrbtc, varblc, ifrqdC, ifmodC,

- iditoc

Length-specified character fields for equivalences ( 1 for length).

character fCSTI $* 12$, fCCSN $* 60$, fCNGAF* 1, fCDCL $1 * 4$, fCDCU $1 * 1$, fCRND $1 * 6$,

fCRNT $1 * 5$, fCDAW $1 * 6$, fCRRC $1 * 20$, fCASI $1 * 1$, fCNGAO 1, fCDCL $2 * 4$, fCDCU $2 * 1$,

- fCRND2 *6, fCRNT2 *5, fCDAW2 *6, fCRRC 2 *20, fCASI $2 * 1$

Real and integer field storage ( $r$ for real, $i$ for integer).

real*4 rCDCL1, rCDCL2

integer $* 4$ iCRND1, iCDAW1, iCRND2, iCDAW2

Nonarrayed maximum field lengths ( 1 for length).

integer *4 1CSTI, 1CCSN, ICNGAF, 1CDCL1, 1CDCU1, 1CRND1, ICRNT1, 1CDAW1, - 1CRRC1, 1CASI1, ICNGAO, ICDCL2 , ICDCU2, 1CRND2, ICRNT2 , ICDAW2, ICRRC2,

- ICASI2

Nonarrayed null indicators for data transfers ( $n$ for null).

integer *2 nCSTI, nCCSN, nCNGAF, nCDCL1, nCDCU1, nCRND1, nCRNT1, nCDAW1, - nCRRC1, nCASI1, nCNGAO, nCDCL2, nCDCU2 , nCRND2, nCRNT2, nCDAW2, nCRRC2,

- nCASI2 
integer *2 mCSTI, mCCSN, mCNGAF, mCDCL1, mCDCU1, mCRND1, mCRNT1, mCDAW1, - mCRRC1, mCÁSI1, mCNGAO, mCDCL2, mCDCU2 , mCRND2, mCRNT2 , mCDAW2, mCRRC2,

\section{Equivalence statements for data transfers.}

equivalence (fidatc(1) (1:12), fCSTI), (ifnulC(1), nCSTI),

(fidatC (2) $(1: 60)$, fCCSN), (i fnulC (2), nCCSN),

- (fidatC (3) $(1: 1)$, fCNGAF), (i fnulC (3), nCNGAF),

- (fidatC (4) (1:4), ECDCL1), (ifnulC (4), nCDCL1),

- (fidatC (5) (1:1), fCDCU1), (ifnulC (5), nCDCU1).

- (fidatC $(6)(1: 6)$, fCRND1), (ifnulC (6), nCRND1).

- (EidatC (7) (1:5), fCRNT1), (i fnulC (7), nCRNT1),

- (fidatC (8) $(1: 6)$, fCDAW1), (i fnulC (8), nCDAW1),

- (fidatC (9) $(1: 20)$, fCRRC1), (ifnulC (9), nCRRC1),

- (fidatC (10) (1:1), fCASI1), (ifnulC (10), nCASI1),

- (fidatC (11) (1:1), fCNGAO), (ifnulC (11), nCNGAO),

- (fidatC (12) $(1: 4)$, fCDCL2), (ifnulC (12), nCDCL2),

- (fidatc (13) (1:1), fCDCU2), (ifnulc (13), nCDCU2),

- (fidatC (14) $(1: 6)$, fCRND2), (ifnulC (14), nCRND2),

- (fidatC (15) $(1: 5)$, fCRNT2), (ifnulC (15), nCRNT2),

- (fidatC $(16)(1: 6)$, fCDAW2), (ifnulC (16), nCDAW2),

- (fidatC (17) $(1: 20)$, fCRRC2), (i fnulC (17), nCRRC2),

- (fidatC (18) $(1: 1)$, ECASI2), (ifnulC (18), nCASI2),

- (mxnocC (1), ICSTI), (ifmodC (1), mCSTI),

- (mxnocC $(2), 1 \mathrm{CCSN}),(i \operatorname{modC}(2), \operatorname{mCCSN})$,

- (mXnocC $(3)$, ICNGAF), (ifmodC (3), mCNGAF),

- (mxnocC (4), ICDCL1), (ifmodC (4), mCDCL1),

- (mxnocC (5), ICDCU1), (ifmodC (5), mCDCU1),

- (mxnocC $(6), 1 C R N D 1)$, (ifmodC (6), mCRND1),

- (mxnocC $(7)$, 1CRNT1), (i $\operatorname{modC}(7)$, mCRNT1),

- (mxnocC (8), ICDAW1), (ifmodC (8), MCDAW1),

- (mxnocC (9), 1CRRC1), (ifmodC (9), mCRRC1),

- (mxnocC $(10)$, ICASI1), (ifmodC (10), mCASI1),

- (mxnOCC (11), 1CNGAO), (ifmodC (11), mCNGAO),

- (mxnocC $(12), 1 C D C L 2),($ ifmodC $(12), \operatorname{mCDCL} 2)$,

- (mxnocC (13), 1CDCU2), (i $\operatorname{mmodC}(13), \operatorname{mCDCU} 2)$,

- (mxnocC (14), 1CRND2), (i $\operatorname{fmodC}(14), \operatorname{mCRND} 2$ ),

- (mxnocC (15), 1CRNT2), (i fmodC (15), mCRNT2),

- (mxnocC (16), ICDAW2), (i imodC (16), mCDAW2),

- (mxnocC (17), 1CRRC2), (ifmodC (17), mCRRC2).

- (mxnocC $(18), 1$ CASI2), (ifmodC $(18), \operatorname{mCASI} 2)$

$\mathrm{C}$
$\mathrm{C}$
$\mathrm{C}$

Database table and attribute names for screen fields.

data atrbtc/
'GROSS ALPHA_BETA_AIR_FILTERS', 'SAMPLE_TRACKING_ID',
' SAMPLE_INFORMATION' ' 'CUSTOMER_SAMPLE_NAME'.
' SAMPLE_INFORMATION' ', NEED_GROSS_ALPHA_BETA_FILTERS',
'GROSS_ALPHA_BETA_AIR_FILTERS' 'DETECTOR_COŨNT_LENGTH' ,
'GROSS_ALPHA_BETA_AIR_FILTERS', ' DETECTOR_COUNT_LENGTH_UNITS',
'GROSS_ALPHA_BETA_AIR_FILTERS' ', 'RESULTS_NEEDED_BY_DATE',
'GROSS_ALPHA_BETA_AIR_FILTERS', 'RESULTS_NEEDED_BY_TIME',
'GROSS_ALPHA_BETA_AIR_FILTERS', 'DATE_ALE_WORK_COMPLETED',
'GROSS_ALPHA_BETA_AIR_FILTERS', 'RESUITS_REPORT_CITATION'
'GROSS_ALPHA_BETA_AIR_FILTERS' ' 'ANY_SPECIAL_INSTRUCTIONS' ,
' SAMPLE_INFORMATION', 'NEED_GROSS_ALPHA_BETA_OTHER', 


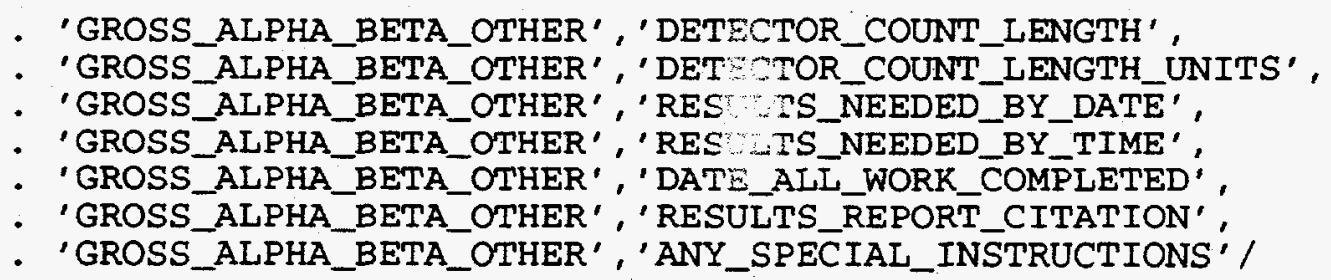

$\mathrm{C}$
$\mathrm{C}$
$\mathrm{C}$

$\mathrm{C}$
$\mathrm{C}$
$\mathrm{C}$

$\mathrm{C}$
$\mathrm{C}$
$\mathrm{C}$

$C$
$C$
$C$
$C$
$C$
$C$
$C$

$\mathrm{C}$
$\mathrm{C}$
$\mathrm{C}$

Program variable names (value and null indicator) for screen fields.

data varblC/' : fCSTI : nCSTI',' : fCCSN: nCCSN',': fCNGAF : ICNGAF',

- ' : rCDCL1 : nCDCL1', ' : fCDCU1 : nCDCU1', : iCRND1 : nCRND1',

- ' : fCRNT1 : nCRNT1', ' : iCDAW1 : nCDAW1', ' : fCRRC1 :nCRRC1',

' : fCASI1 : nCASI1', ' : fCNGAO: nCNGAO',' : rCDCL2 : nCDCL2',

' : fCDCU2 : nCDCU2' ' : iCRND2 : nCRND2', ' : fCRNT2 : nCRNT2',

. ' : ICDAW2 : nCDAW2', ' : fCRRC2 : nCRRC2', ' fCASI2 : nCASI2',

Special instruction screen's'field descriptors and field sizes.

character $* 80$ fieldI (32), fidatI(32)

character $* 30$ atrbt $I(2,5)$

character $* 14$ varblI (5)

character*1 fitypI(32)

integer $* 4$ fdlenI (32), fdrowI (32), fdcolI (32), ficolI (32), mxnocI (32),

. fdrndI (3,32), firndI $(3,32)$, !Field renditions (add/update/search).

- numfdI/32/ !The number of fields.

integer ${ }^{2}$ ifnulI $(32)$,

- ifrqdI (32) $132 * 0 \%$ ! Points to if-required dependent field.

- iditoI (32)/32*0/ ! Points to allowed field for dittoing.

logical ifmodI(32) ! Has a field been modified in an Update?

common /dbdatI/ fieldI, fidatI, fdlenI, fdrowI, fdcolI, ficolI, mxnocI,

- fdrndI, firndI, numfdI, fitypI, ifnulI, atrbt I, varblI, ifrqdI, ifmodI,

- iditoI

Length-specified character fields for equivalences (f for field).

character. fISTI* $12, \mathrm{fICSN} * 60, \mathrm{fIOS} 1 * 16, \mathrm{fISI} 1 * 50, \mathrm{fIOS} 2 * 16$, fISI 2 * 50 ,

- fIOS $3 * 16, \mathrm{fISI} 3 * 50, \mathrm{fIOS} 4 * 16, \mathrm{fISI} 4 * 50, \mathrm{fIOS} 5 * 16, \mathrm{fISI} 5 * 50, \mathrm{fIOS} 6 * 16$,

- fISI6*50, fIOS $7 * 16$, fISI $7 * 50$, fIOS $8 * 16$, fISI $8 * 50$, fIOS 9 *16, fISI9*50,

- fIOS $10 * 16, f I S I 10 * 50, f I O S 11 * 16, f I S I 11 * 50, f I O S 12 * 16, f I S I 12 * 50$,

- fIOS13*-, EISI13*50, fIOS14*16, EISI14*50, fIOS15*16, EISI $15 * 50$,

- fIOS1t*16, fISI 1 *50 ! And spares for inserts and fetchs.

Integer field storage ( $i$ for integer).

integer*4 siline !Declared in the main routine.

Nonarrayed maximum field lengths ( 1 for length).

integer* 4 IISTI, IICSN, IIOS1, IISI1, 1 IOS2, IISI2, 1IOS3, 1 ISI3, 1 IOS4,

- IISI4, IIOS5, IISI5, 1 IOS6, IISI6, IIOS7, IISI7, IIOS8, 1 ISI 8,1 IOS9,

- IISI9, IIOS10, IISI10, IIOS11, IISI11, IIOS12, IISI12, IIOS13, IISI13,

- 1IOS14,1ISI14,1IOS15,IISI15,

- lIOS1t,1ISI1t !And spares for inserts and fetchs.

Nonarrayed null indicators for data transfers (n for null).

integer 2 nISTI, nICSN, nIOS1, nISI1, nIOS2, nISI2, nIOS3, nISI3, nIOS4,

- nISI4, nIOS5, nISI5, nIOS6, nISI6, nIOS7, nISI7, nIOS8, nISI8, nIOS9,

. nISI9, nIOS10, nISI10, nIOS11, nISI11, nIOS12, nISI12, nIOS13, nISI13, 
- nIOS14,nISI14, nIOS15, nISI15,

- nIOS1t,nISIIt !And spares for inserts and fetchs.

C

C

$\mathrm{C}$

C
Nonarrayed if-modified indicators (m for modified).

integer ${ }^{2}$ mISTI, mICSN, mIOS1, mISI1, mIOS2, mISI2, mIOS3, mISI3, mIOS4,

- mISI4, mIOS5, mISI5, mIOS6, mISI6, mIOS7, mISI7, mIOS8, mISI8, mIOS9,

- mISI9, mIOS10, mISI10, mIOS11, mISI11, mIOS12, mISI12, mIOS13, mISI13,

- mIOS14, MISI14, mIOS15, mISI15.

- mIOS1t,mISI1t !And spares for inserts and fetchs.

Equivalence statements for data transfers.

equivalence (fidatI(1) (1:12), fISTI), (ifnulI(1), nISTI),

(fidatI $(2)(1: 60)$, ICSN), (ifnulI (2), nICSN),

- (fidatI (3) $(1: 16)$, fIOS1), (ifnulI(3), nIOS1),

(fidatI (4) (1:50), fISI1), (ifnulI (4), nISI1),

(fidatI (5) (1:16), fIOS2), (ifnulI (5), nIOS2),

- (fidatI (6) (1:50), fISI2), (ifnuII (6),nISI2),

- (fidatI (7) (1:16), fIOS3), (i fnulI (7),nIOS3),

- (fidatI (8) (1:50), fISI3), (ifnulI (8),nISI3),

- (fidatI (9) (1:16), fIOS4), (ifnulI (9), nIOS4),

- (fidatI(10) (1:50), fISI4), (ifnulI(10),nISI4),

(fidatI (11) (1:16), fIOS5), (ifnulI(11), nIOS5),

(fidatI (12) (1:50), fISI5), (ifnulI(12),nISI5),

- (fidatI (13) (1:16), fIOS6), (ifnulI (13),nIOS6),

- (fidatI (14) (1:50), fISI6), (ifnulI (14),nISI6),

- (fidatI (15) (1:16), fIOS7), (ifnulI (15), nIOS7),

- (fidatI (16) (1:50), fISI7), (ifnulI (16), nISI7),

- (fidatI (17) (1:16), fIOS8), (ifnulI (17), nIOS8),

- (fidatI (18) (1:50), fISI8), (ifnu1I (18),nISI8),

- (fidatI (19) (1:16), fIOS9), (ifnulI (19), nIOS9),

- (fidatI (20) (1:50), fISI9), (ifnulI (20),nISI9),

- (fidatI (21) (1:16), fIOS10), (ifnulI(21),nIOS10),

- (fidatI (22) $(1 ; 50)$, fISI10), (ifnulI (22), nISI10),

- (fidatI (23) (1:16), fIOS11), (ifnulI(23),nIOS11),

- (fidatI (24) (1:50), EISI11), (ifnulI (24), nISI11),

- (fidatI (25) (1:16), fIOS12), (ifnulI(25), nIOS12),

- (fidatI (26) (1:50), EISI12), (ifnulI (26),nISI12),

- (fidatI (27) (1:16), fIOS13), (ifnulI(27), nIOS13),

- (fidatI (28) (1:50), fISI13), (ifnulI (28), nISI13),

- (fidatI (29) (1:16), fIOS14), (ifnuII(29),nIOS14),

- (fidatI (30) (1:50), fISI14), (i fnulI(30), nISI14),

- (fidatI (31) (1:16), fIOS15), (ifnulI (31), nIOS15),

- (fidatI (32) $(1: 50)$, fISI15), (i fnulI (32),nISI15),

- (mxnocI (1), IISTI), (ifmodI (1), mISTI),

- (mxnocI $(2), 1$ ICSN), (ifmodI (2), mICSN),

- (mxnocI (3), IIOS1), (ifmodI (3), mIOS1),

- (mxnocI (4), IISI1), (ifmodI (4),mISI1),

- (mxnocI (5), I IOS2), (ifmodI (5), mIOS2).

- (mxnocI $(6)$, IISI2), (ifmodI (6), mISI2),

- (mxnocI (7), 1 IOs3), (ifmodI (7), mIOS3),

- (mXnocI (8), IISI3), (ifmodI (8), mISI3),

- (mxnocI $(9), 1$ IOS4), (ifmodI (9), mIOS4),

- (mxnocI (10), IISI4), (ifmodI (10), mISI4),

- (mxnocI (11), 1 IOS5), (i fmodI (11), mIOS5),

- (mxnocI (12), IISI5), (ifmodI (12), mISI5),

- (mxnocI (13), lIOS6), (ifmodI (13), mIOS6).

- (mxnocI (14), iISI6), (ifmodI (14), mISI6),

- (mxnocI (15), 1IOS7), (ifmodI (15), mIOS7), 
- (mxnocI (16), IISI7), (ifmodI (16), mISI7),

(mxnocI $(17), 1$ IOS8), (ifmodI (17), mIOS8),

- (mxnocI (18), 1ISI8), (ifmodI (18), mISI8),

- (mxnocI (19), IIOS9), (ifmodI (19), mIOS9),

- (mxnocI (20), IISI9), (ifmodI (20),mISI9),

- (mxnocI (21), IIOS10), (ifmodI (21), mIOS10),

- (mxnocI (22), IISI10), (ifmodI (22), mISI10),

- (mxnocI (23), 1IOS11), (ifmodI (23), mIOS11),

- (mxnocI (24), IISI11), (ifmodI (24), mISI11),

- (mxnocI (25), 1 IOS12), (ifmodI (25), mIOs12),

- (mxnocI (26), IISI12), (ifmodi (26), mISI12),

- (mxnocI (27), IIOS13), (ifmodI (27), mIOS13),

$\therefore(\operatorname{mxnocI}(28), \operatorname{lISI13}),(\operatorname{ifmodI}(28), \operatorname{mISI13})$,

- (mxnocI (29), IIOS14), (ifmodI (29), mIOS14),

- (mxnocI (30), IISI14), (ifmodI (30), mISI14),

- (mxnocI (31), 1IOS15), (ifmodI (31), mIOS15),

- (mxnocI (32), IISI 15), (i $\bmod (32), \operatorname{mISI15})$

$\mathrm{C}$
$\mathrm{C}$
$\mathrm{C}$

Database table and attribute names for screen fields.

data atrbtI/

'SPECIAL INSTRUCTIONS', 'SAMPLE_TRACKING_ID',

- 'SAMPLE_INFORMATION' ,' CUSTOMER_SAMPLE_NAME',

- 'SPECIAI_INSTRUCTIONS', 'ORIGIN_OF_SPECIAL_INSTRUCTION',

- 'SPECIAL_INSTRUCTIONS', ' SPECIAL_IÑSTRUCTION',

- 'SPECIAL_INSTRUCTIONS', 'SPECIAL_INSTRUCTION_LINE' /

C

C

C

C

$\mathrm{C}$

C

C

C

$\mathrm{C}$

C

C

program variable names (value and null indicator) for screen fields.

data varbII/':EISTI:nISTI',':fICSN:nICSN',':fIOSIt:nIOS1',

. ':fISI1t:nISI1',':siline:ifnsil'/

Possessor screen's field descriptors and field sizes.

character $\star 80$ fieldT(23), fidat $T(23)$

character $* 30$ atrbt $T(2,9)$

character *14 varblT (9)

character*1 fitypt (23)

integer 4 fdlent (23), fdrowt (23), fdcolT (23), ficolT (23), mxnoct (23),

. fdrndT (3,23), firndT $(3,23)$, ! Field renditions (add/update/search).

- numfdT/23/ ! The number of fields.

integer ${ }_{2}$ ifnulT(23), ! Null indicator (-1 if null, 0 otherwise).

- ifrqdT (23) $/ 23 * 0 \%$, points to if-required dependent field.

- iditot (23) $/ 23 * 0 /$ ! Points to allowed field for dittoing.

logical ifmodT(23) ! Has a field been modified in an Update?

common /dbdatT/ fieldT, fidat T, fdlenT, fdrowT, fdcolT, ficolT, mxnocT,

- fdrndT, firndT, numfdT, fitypT, ifnulT, atrbt $T$, varblT, ifrodT, i fmodT,

- iditoT

Length-specified character fields for equivalences ( $f$ for field).

character fTSTI*12, fTCSN*60, fTPSN1 *3, fTPN1 25, fTPPN1 *12, fTPA 1 * 60 ,

. ITPPR $1 * 50, \mathrm{ITDAB} 1 * 6, \mathrm{ITDRB} 1 * 6, \mathrm{ITPSN} 2 * 3, \mathrm{ITPN} 2 * 25$, ITPPN2 2 12, ITPA $2 * 60$,

- fTPPR $2 * 50$, fTDAB $2 * 6$, fTDRB2 $* 6$, fTPSN $3 * 3$, fTPN $3 * 25$, fTPPN $3 * 12$, ITPA $3 * 60$,

- ITPPR $3 * 50, \mathrm{fTDAB} 3 * 6, \mathrm{fTDRB} 3 * 6$,

- ETPN1t *25, fTPPN1t*i2, fTPA1t*60, ETPPR it*50 ! Temporary fields.

Integer field storage ( $i$ for integer).

integer * 4 iTPSN1, iTDAB1, iTDRB1 
integer*4 1TSTI, 1TCSN, 1TPSN1, 1TPN1, 1TPPN1, 1TPA1, 1TPPR1, 1TDAB1, - 1TDRB1, ITPSN2, ITPN2, ITPPN2, ITPA2, ITPPR2, 1TDAB2, 1TDRB2, ITPSN3, - 1TPN3, 1TPPN3, 1TPA3, 1TPPR3, 1TDAB3, 1TDRB3

Nonarrayed null indicators for data transfers (n for null).

integer *2 nTSTI, nTCSN, nTPSN1, nTPN1, nTPPN1, nTPA1, nTPPR1, nTDAB1, . nTDRB1, nTPSN2 , nTPN2, nTPPN2, nTPA2, nTPPR2, nTDAB2, nTDRB2, nTPSN3, - nTPN3, nTPPN3, nTPA3, nTPPR3, nTDAB3, nTDRB3

$\mathrm{C}$

Nonarrayed if-modified indicators (m for modified).

integer*2 mTSTI, mTCSN, mTPSN1, mTPN1, mTPPN1, mTPA1, mTPPR1, mTDAB1, - mTDRB1 , mTPSN2 , mTPN2 , mTPPN2 , mTPA2 , mTPPR2 , mTDAB2 , mTDRB2 , mTPSN3, - mTPN3, mTPPN3 , mTPA3 , mTPPR3, mTDAB3, mTDRB3

equivalence (fidatT(1) (1:12), fTSTI), (ifnulT(1), nTSTI),

(fidatT (2) (1:60), fTCSN), (ifnulT (2), nTCSN),

- (EidatT (3) (1:3), fTPSN1), (ifnulT (3),nTPSN1),

(EidatT (4) (1:25), ETPN1), (ifnulT (4), nTPN1),

- (fidatt (5) (1:12), fTPPN1), (ifnulT (5) , nTPPN1),

- (fidatt (6) (1:60), fTPA1), (ifnult (6),nTPA1),

- (fidatT (7) (1:50), fTPPR1), (ifnult (7), nTPPR1),

- (fidatt (8) (1:6), fTDAB1), (ifnulT(8),nTDAB1),

- (fidatt (9) (1:6), fTDRB1), (ifnulT (9),nTDRB1).

- (fidatt (10) (1:3), ETPSN2), (ifnulT (10), nTPSN2),

- (fidatT (11) (1:25), fTPN2), (ifnulT (11), nTPN2),

- (fidatT (12) (1:12), fTPPN2), (ifnulT (12), nTPPN2),

- (fidatt (13) (1:60), fTPA2), (ifnulT (13), nTPA2),

- (fidatT (14) (1:50), fTPPR2), (ifnu1T (14), nTPPR2),

- (fidatT (15) (1:6), ETDAB2), (ifnulT (15), nTDAB2),

- (fidatT (16) (1:6), fTDRB2), (ifnulT (16), nTDRB2),

- (fidatT (17) (1:3), ETPSN3), (ifnulT (17), nTPSN3),

- (fidatT (18) (1:25), fTPN3), (ifnulT (18), nTPN3).

- (fidatT (19) (1:12), fTPPN3), (ifnu1T(19), nTPPN3),

- (fidatT (20) (1:60), fTPA3), (ifnult (20), nTPA3),

- (fidatT (21) (1:50), ETPPR3), (ifnulT (21), nTPPR3),

- (fidatt $(22)(1: 6)$, fTDAB3), (ifnult (22), nTDAB3).

- (fidatT (23) (1:6), ETDRB3), (ifnulT (23), nTDRB3),

- (mxnoct (1), 1TSTI), (i $\bmod$ (1), mTSTI),

- (mxnoct (2), 1TCSN), (ifmodT (2), mTCSN),

- (mxnoct (3), ITPSN1), (ifmodT (3), mTPSN1),

- (mXnoct (4),1TPN1), (ifmodT (4), mTPN1),

- (mxnoct (5), ITPPN1), (ifmodT (5), mTPPN1),

- (mxnoct $(6), 1 \mathrm{TPA}),(\operatorname{i\operatorname {modT}}(6), \mathrm{mTPA} 1)$,

- (mxnoct (7), 1TPPR1), (ifmodT (7), mTPPR1),

- (mXnoct (8), ITDAB1), (ifmodT (8), mTDAB1),

- (mxnoct (9), 1TDRB1), (ifmodT (9), mTDRB1),

- (mxnoct (10), 1TPSN2), (i $\operatorname{modT}(10)$, mTPSN2),

- (mxnoct (11), 1TPN2), (ifmodT (11), mTPN2),

- (mxnoct (12), 1TPPN2), (i $\operatorname{modT}(12), \operatorname{mTPPN2})$,

- (mxnoct (13), 1TPA2), (ifmodT (13), mTPA2),

- (mxnoct (14), 1TPPR2), (ifmodT (14), mTPPR2),

- (mxnoct (15), 1TDAB2), (i $\operatorname{modT}(15)$, mTDAB2),

- (mxnoct (16), 1TDRB2), (i fmodT (16), mTDRB2), 
C

- (mxnoct $(17), 1$ TPSN3), (ifmodT $(17), \operatorname{mTPSN} 3)$,

- (mxnoct $(18), 1$ TPN3), (i $\operatorname{imodT}(18), \mathrm{mTPN} 3)$,

- (mxnoct (19), 1TPPN3), (i $\bmod (19)$, mTPPN3),

- (mxnoct (20), 1TPA3), (ifmodT (20), mTPA3) ,

- (mxnoct $(21), 1$ TPPR3), (ifmodT (21), mTPPR3) ,

- (mxnoct (22), lTDAB3), (ifmodT (22), mTDAB3),

- (mxnocT (23), 1TDRB3), (i $\operatorname{modT}(23), \operatorname{mTDRB} 3)$

C

Database table and attribute names for screen fields.

data atrbtT/

- 'TRACKING' , 'SAMPLE_TRACKING_ID',

- 'SAMPLE_INFORMATION' ' 'CUSTOMER_SAMPLE_NAME' ,

- 'TRACKING' , ' POSSESSOR_SEQUENCE_NUMBER' ,

- 'TRACKING', 'POSSESSOR_NAME',

. 'TRACKING', 'POSSESSOR_PHONE_NUMBER' ,

- 'TRACKING' ,' POSSESSOR_ADDRESS' ,

- 'TRACKING', 'POSSESSOR_POSSESSION_REASON' ,

- 'TRACKING' , 'DATE_ACCEPTED_BY_POSSESSOR' ,

- 'TRACKING', 'DATE_RELINQUISHED_BY_POSSESSOR' /

C

C

C

C

$\mathrm{C}$

C

C

$\mathrm{C}$

C

$\mathrm{C}$

C

Program variable names (value and null indicator) for screen fields.

data varblt/':fTSTI:nTSTI',': fTCSN: nTCSN' ', : iTPSN1:nTPSN1',

. ' : fTPN1 : nTPN1' ' : fTPPN1 : nTPPN1' ' : fTPA1 :nTPA1', ': fTPPR1:nTPPR1',

. ' : iTDAB1 : nTDAB1', ': iTDRB1 : nTDRB1'/

Possessor name list field descriptors and sizes.

character $* 80$ fieldN $(4,15)$

integer *4 fdlenN (4,15), fdrown (15), fdcolN (15), ficolN (15),

- numfdN !The number of fields.

common / IsdatN/ fieldN, fdlenN, fdrown, fdcoln, ficolN, numfdN

For the Main screen, . .

Set defaults for display field renditions now so they can be modified with each field as necessary. Set all input fields to blanks.

do $i=1$, numfdM

fidat $M(i)=$ blanks

do $j=1,3$ ! 1 for adding, 2 for updating, and 3 for searching. fdrndM $(j, i)=$ foptnl enddo

enddo

fieldM(1)='Tracking ID:'

fdlenM $(1)=12$

$\operatorname{farow}(1)=2$

$\operatorname{fdcolm}(1)=1$

$f i \operatorname{col} M(1)=15$

$\operatorname{mxnOCM}(1)=12$

fitypM $(1)=' 1$ '

fieldM (2) ='Date Entered: '

fdlenM $(2)=13$

fdrowM $(2)=2$

$\operatorname{facol} M(2)=31$

$f i \operatorname{col} M(2)=46$

$\operatorname{mxnocM}(2)=6$ 
C

fitypM(2) ='d'

do $i=1,2$

do $j=1,2$ ! Fixed when adding and updating. fdrndM $(j, i)=f$ fixed enddo

C enddo

fieldM(3) =' Completed:'

fdlenM $(3)=10$

farowM $(3)=2$

$\operatorname{fdcolm}(3)=56$

$\mathrm{ficolm}(3)=68$

$\operatorname{mxnocM}(3)=6$

C

fitypM(3) ='d'

fieldm(4)='Customer' 's Sample ID:'

fdlenM $(4)=22$

$\operatorname{farow} M(4)=3$

$\operatorname{facol} M(4)=4$

$\mathrm{ficolm}(4)=27$

$\operatorname{mxnocM}(4)=12$

C

fitypM $(4)=' a '$

fieldM(5)='Desired Completion Date:'

fdlenM $(5)=24$

fdrowm $(5)=3$

$\operatorname{fdcolm}(5)=45$

$f i \operatorname{col} M(5)=71$

$\operatorname{mxnocm}(5)=6$

fitypM $(5)=$ ' $d$ '

do $j=1,2$ ! Required when adding and updating. $\operatorname{fdrndM}(j, 5)=$ freqd

C enddo

fieldm $(6)=$ 'Project:'

fdlenM $(6)=8$

$\operatorname{farowM}(6)=4$

$\operatorname{fdcol} M(6)=4$

$\operatorname{ficolm}(6)=14$

$\operatorname{mxnocM}(6)=40$

fitypm $(6)=' a^{\prime}$

C

fieldM(7)='Charge \#:'

fdlenM $(7)=9$

fdrowM $(7)=4$

$\operatorname{fdcolM}(7)=58$

ficolm $(7)=69$

$\operatorname{mxnOCM}(7)=9$

C

fitypM $(7)=' 1$ '

fieldM $(8)=$ 'Sample Name:'

fdlenM $(8)=12$

fdrowM $(8)=5$

$\operatorname{facol} M(8)=4$

$\mathrm{ficolm}(8)=18$

$\operatorname{mxnocM}(8)=60$

fitypM $(8)=' a$ '

C

iditoM $(8)=4$ 
fieldM(9) =' Sample Type:'

fdlenM $(9)=12$

farowm $(9)=6$

$\mathrm{fdcolM}(9)=4$

ficolm $(9)=18$

$\operatorname{mxnocm}(9)=10$

C

fitypM $(9)=$ 'a'

fieldM(10)='Sample Size:'

falenM $(10)=12$

fdrowm $(10)=6$

$\operatorname{facolm}(10)=32$

$\mathrm{ficolM}(10)=46$

$\operatorname{mxnocm}(10)=5$

C

fitypM $(10)=$ ' $\mathrm{n}$ '

C

fieldM(11)='Size Units:'

fdlenM $(11)=11$

fdrowm $(11)=6$

fdcolm $(11)=55$

fiColM $(11)=68$

$\operatorname{mxnOCM}(11)=5$

fitypm $(11)=' a$ '

fieldm(12)='Collection Date:'

fdleng $(12)=16$

fdrowm $(12)=7$

$\operatorname{fdcolm}(12)=4$

$\operatorname{ficolm}(12)=22$

$\operatorname{mxnOCM}(12)=6$

C

fitypM $(12)=$ ' $d$ '

do $i=7,12$

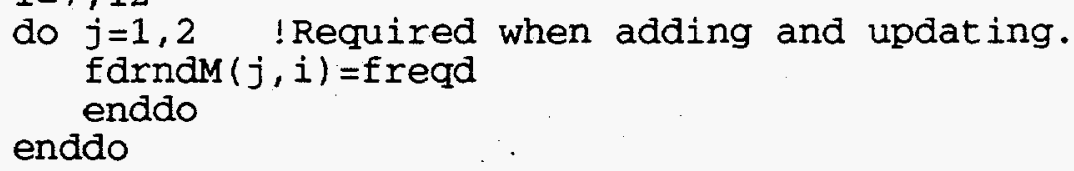

C

fieldm $(13)=$ 'Time: '

fdlenM $(13)=5$

farowm $(13)=7$

$\operatorname{fdcolm}(13)=32$

ficolm $(13)=39$

$\operatorname{mxnocM}(13)=5$

fitypm $(13)=$ ' $t$ '

C

fieldM $(14)=$ 'Special Instructions?'

fdlenM $(14)=21$

farowm $(14)=7$

$\operatorname{fdcolm}(14)=48$

$f i \operatorname{col} M(14)=71$

$\operatorname{mxnocM}(14)=1$

C

fitypM $(14)=$ ' ?'

fieldM(15)='HP Surveyed?'

fdlenM $(15)=12$

farowM $(15)=8$

fdcolm $(15)=4$

ficolm $(15)=18$

$\operatorname{mxnocm}(15)=1$ 
fitypM (15)='?'

C

ifrqdM $(15)=16$

fieldM(16)='If Yes, Sample Activity (mrem/h) :' fdlenM $(16)=33$

fdrowM $(16)=8$

$\operatorname{fdcolm}(16)=23$

$\mathrm{ficolM}(16)=58$

$\operatorname{mxnocM}(16)=6$

fitypM $(16)=$ 'n'

C

ifrqdM $(16)=15$

fielaM $(17)=$ 'Submitter:'

fdlenM $(17)=10$

fdrowM $(17)=9$

$\operatorname{fdcolm}(17)=1$

$\mathrm{fiColM}(17)=21$

$\operatorname{mxnocM}(17)=25$

C

fitypM $(17)=$ ' $a$ '

lincnt $=7$

do $i=18,30,3$

lincnt $=1$ incnt +2

fieldM(i)='Phone Number:'

fdlenM $(i)=13$

fdrow $(i)=1$ incnt

$f d \operatorname{col} M(i)=50$

$f i \operatorname{col} M(i)=65$

$\operatorname{mxnocM}(i)=12$

fitypM $(i)=' a$ '

ifrqdM $(i)=i-1$

if (i.ne. 18) iditom( $i)=i-3$

C

fieldM $(i+1)=$ 'Address :'

fdlen $(i+1)=8$

fdrowm $(i+1)=1$ incnt +1

$\operatorname{fdcolm}(i+1)=4$

$f i \operatorname{col} M(i+1)=14$

$\operatorname{mxnocM}(i+1)=60$

fitypM $(i+1)=$ ' $a$ '

ifrgdM $(i+1)=i-1$

if $(i . n e .18)$ iditom $(i+1)=i-2$

C enddo

do $i=17,19$

do $j=1,2$ ! Required when adding and updating. $\operatorname{farndM}(j, i)=$ freqd

enddo enddo

C

fieldm $(20)=$ 'Technical contact:'.

falen $(20)=18$

fdrow $(20)=11$

$\operatorname{fdcolm}(20)=1$

$\operatorname{ficolm}(20)=21$

$\operatorname{mxnocM}(20)=25$

fitypM $(20)=' a$ '

C

iditom $(20)=17$

fieldm $(23)=$ 'Send Results To:' 


$$
\begin{aligned}
& \text { fdleng }(23)=16 \\
& \operatorname{fdrowm}(23)=13 \\
& \operatorname{fdcolm}(23)=1 \\
& \operatorname{ficolm}(23)=21 \\
& \operatorname{mxnocm}(23)=25 \\
& \text { fitypm }(23)=a^{\prime} \\
& \text { iditom }(23)=20
\end{aligned}
$$

C

C

$$
\begin{aligned}
& \text { fieldM }(26)=\text { 'Sample Pickup By:' } \\
& \text { fdlenM }(26)=17 \\
& \text { fdrowM }(26)=15 \\
& \text { fdcolM }(26)=1 \\
& \text { ficolm }(26)=21 \\
& \text { mxnocm }(26)=25 \\
& \text { fitypM }(26)=' a \\
& \text { iditom }(26)=23
\end{aligned}
$$

C

do $i=23,28$

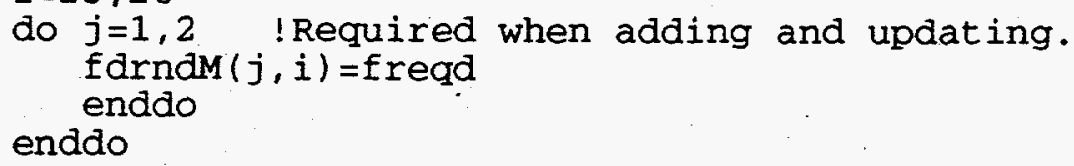

fieldM(29) ='Current Possessor:'

fdlenM $(29)=18$

fdrow $(29)=17$

fdcolm $(29)=1$

$\mathrm{fi} \operatorname{col} M(29)=21$

$\operatorname{mxnOcM}(29)=25$

$\mathrm{C}$

fitypm $(29)=' a$ '

do $i=29,31$

fdrndM $(1, i)=f f i x e d$ !Fixed when adding and updating.

fdrndM $(2, i)=$ ffixed

enddo

C

fieldM $(32)=$ 'Chain of Custody Number:'

fdlenM $(32)=24$

$\operatorname{farowM}(32)=19$

$\operatorname{fdcolm}(32)=1$

$f i \operatorname{col} M(32)=27$

$\operatorname{mxnocm}(32)=10$

C

$$
\text { fitypM }(32)=' a \text { ' }
$$

fieldM(33) ='Analyses Needed: Gross Alpha-Beta?'

fdlenM $(33)=35$

fdrowM $(33)=20$

$\operatorname{fdcolm}(33)=1$

$f i \operatorname{col} M(33)=38$

$\operatorname{mxnocM}(33)=1$

C

fitypM $(33)=$ '?'

fieldM (34) ='Alpha?'

fdlenM $(34)=6$

fdrowM $(34)=20$

$\operatorname{fdcolm}(34)=43$

$\mathrm{fiCOIM}(34)=51$

$\operatorname{mxnocM}(34)=1$

C

fitypM $(34)=$ '?' 


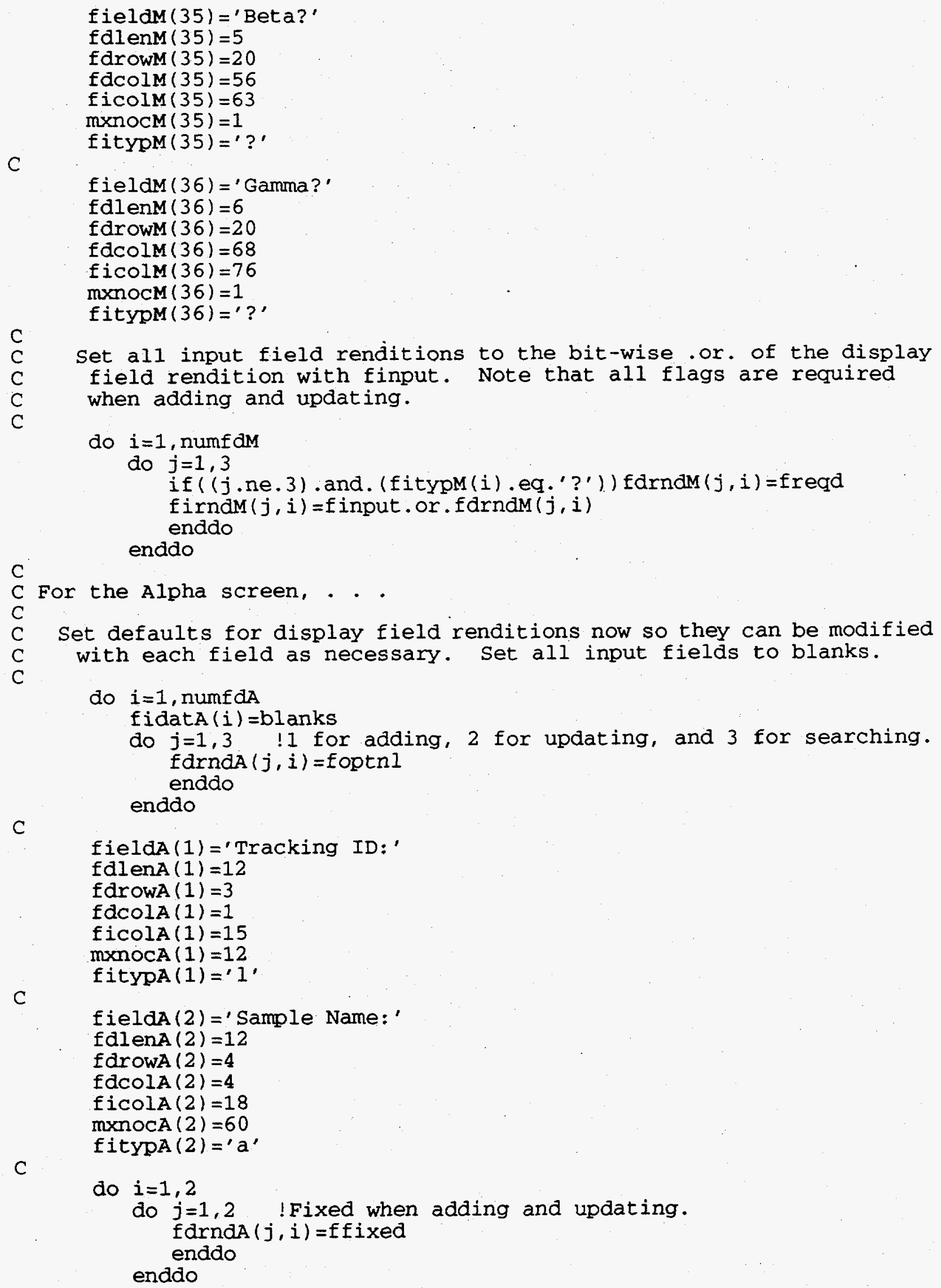

$\mathrm{C}$

C For the Alpha screen, . .

C Set defaults for display field renditions now so they can be modified C with each field as necessary. Set all input fields to blanks.

C

do $i=1$, numfdA

fidat $A(i)=b l a n k s$

do $j=1,3$ ! 1 for adding, 2 for updating, and 3 for searching. $\operatorname{farndA}(j, i)=$ foptnl enddo

C enddo

fieldA $(1)=$ 'Tracking ID:'

fdlenA $(1)=12$

fdrowA $(1)=3$

fdcola $(1)=1$

$\mathrm{ficolA}(1)=15$

$\operatorname{mxnocA}(1)=12$

C

fitypa $(1)=' 1$,

fieldA $(2)=$ ' Sample Name:'

fdlenA $(2)=12$

fdrowA $(2)=4$

$\mathrm{fdcolA}(2)=4$

$f i \operatorname{colA}(2)=18$

$\operatorname{mxnOCA}(2)=60$

C

fitypA $(2)=' a$ '

do $i=1,2$

do $j=1,2$ ! Fixed when adding and updating. fdrndA $(j, i)=f$ fixed enddo

enddo 
C

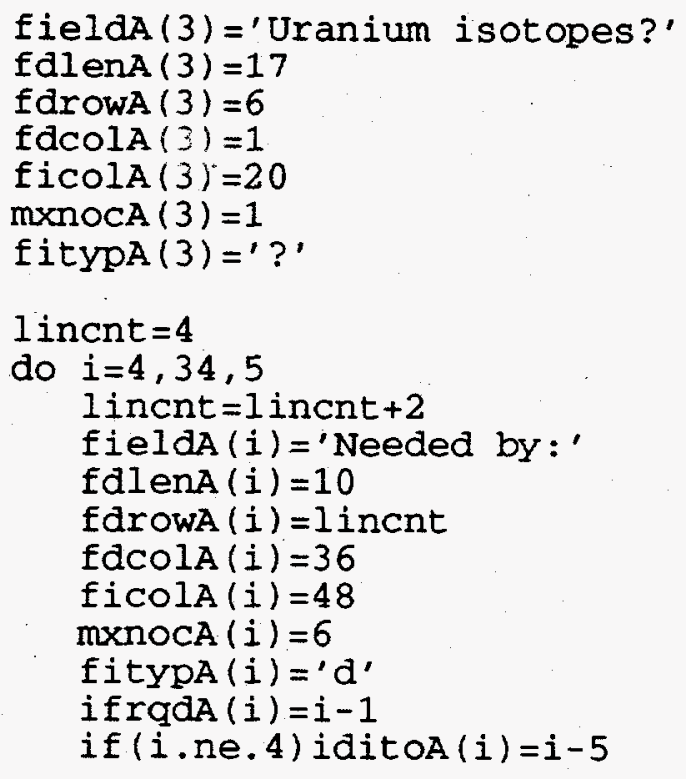

C

fieldA $(i+1)=$ ' Completed:'

fdlenA $(i+1)=10$

fdrowA $(i+1)=1$ incnt

facolA $(i+1)=58$

$f i \operatorname{col} A(i+1)=70$

$\operatorname{mxnOCA}(i+1)=6$

fitypA $(i+1)=$ 'd'

ifrgdA $(i+1)=i+2$

if $(i . n e .4)$ idito $(i+1)=i-4$

C

fieldA $(i+2)=$ 'Results report citation:'

fdlenA $(i+2)=24$

fdrowA $(i+2)=1$ incnt +1

$\operatorname{fdcolA}(i+2)=4$

$f i \operatorname{col} A(i+2)=30$

$\operatorname{mxnOCA}(i+2)=20$

fitypa $(i+2)=' a$ '

ifrgda $(i+2)=i+1$

if $(i$.ne. 4$)$ iditod $(i+2)=i-3$

C

fieldA $(i+3)=$ 'Special Instructions?'

fdlenA $(i+3)=21$

farowA $(i+3)=1$ incnt +1

$\operatorname{fdcolA}(i+3)=54$

ficolA $(i+3)=77$

$\operatorname{mxnOCA}(i+3)=1$

fitypa $(i+3)=$ '?'

ifrgda $(i+3)=i-1$

if $(i$. ne. 4$)$ iditoA $(i+3)=i-2$

C

enddo

fieldA (8) = 'Thorium isotopes?'

fdlenA $(8)=17$

fdrowA $(8)=8$

fdcola $(8)=1$

$f i \operatorname{col} A(8)=20$

$\operatorname{m\times nOCA}(8)=1$

fitypA (8) ='?' 
C

fieldA $(13)=$ 'Plutonium isotopes?'

fdlenA $(13)=19$

fdrowA $(13)=10$

fdcola $(13)=1$

$\mathrm{fiColA}(13)=22$

$\operatorname{mxnOCA}(13)=1$

C

fitypA $(13)='$ ?'

fieldA (18) ='Am-241 separate from Pu-238?'

fdlenA $(18)=28$

fdrowA $(18)=12$

$\operatorname{fdcolA}(18)=1$

$\mathrm{ficolA}(18)=31$

$\operatorname{mXnOCA}(18)=1$

C

fitypA $(18)={ }^{\prime}$ ?'

fieldA (23) ='Am-241 combined with Pu-238?'

falenA $(23)=28$

farowA $(23)=14$

fdcolA $(23)=1$

ficolA $(23)=31$

mXnOcA $(23)=1$

C

fitypA $(23)=$ '?'

fielda (28)='Total Spectrometric Alpha?'

fdlenA $(28)=26$

fdrowA $(28)=16$

fdcolA $(28)=1$

ficolA $(28)=29$

$\operatorname{mxnOCA}(28)=1$

C

fitypA $(28)=$ '?'

fieldA $(33)=$ 'Other?'

fdlenA $(33)=6$

farowA (33) $=18$

$\mathrm{fdcolA}(33)=1$

$\mathrm{ficolA}(33)=9$

$\operatorname{mxnOCA}(33)=1$

fitypA $(33)={ }^{\prime}$ ?'

C

C

Set ali input field renditions to the bit-wise or. of the display field rendition with finput. Note that some flags are required when adding and updating.

$\mathrm{C}$

do $i=1$, numfdA

do $j=1,3$

if ( (j.ne. 3) . and. (fitypa (i) .eq.'?') . and.

(fieldA (i).ne.'Special Instructions?') ) fdrndA $(j, i)=$ freqd firndA $(j, i)=f i n p u t$.or. $f$ drndA $(j, i)$

enddo enddo

C

C For the Beta screen, . .

C

C Set defaults for display field renditions now so they can be modified

C with each field as necessary. Set all input fields to blanks.

$\mathrm{C}$

do $i=1$, numfdB

fidat $B(i)=b l$ anks 
C

do $j=1,3$ ! 1 for adding, 2 for ypdating, and 3 for searching. farndB $(j, i)=$ foptnl

enddo enddo

fieldB $(1)=$ 'Tracking ID:'

fdlen $B(1)=12$

farowB $(1)=2$

$\operatorname{fdcolB}(1)=1$

ficolB $(1)=15$

$\operatorname{mxnocB}(1)=12$

C

$$
\text { fitypB }(1)=' 1 \text { ' }
$$

fieldB $(2)=$ 'Sample Name:'

fdlenB $(2)=12$

fdrowB $(2)=3$

fdcolB $(2)=4$

$\mathrm{ficolB}(2)=18$

$\operatorname{mxnocB}(2)=60$

C

fitypB $(2)=' a$ '

C

do $i=1,2$

do $j=1,2$ ! Fixed when adding and updating. fdrndB $(j, i)=$ ffixed endao

enddo

fieldB $(3)=$ ' Strontium-90?'

fdlen $B(3)=13$

farowB $(3)=5$

$\operatorname{fdcolB}(3)=1$

$f i \operatorname{colB}(3)=16$

$\operatorname{mxnOCB}(3)=1$

C

fitypB $(3)=$ '?'

lincnt $=2$

do $i=4,28,8$

lincnt $=1$ incnt +3

fieldB $(i)=$ 'Length of count:'

fdlenB $(i)=16$

fdrowB $(i)=1$ incnt

$f d \operatorname{colB}(i)=29$

$f i \operatorname{colB}(i)=47$

$\operatorname{mxnOCB}(i)=4$

fitypB $(i)=' n$ '

ifrqdB $(i)=i+1$

if (i.ne.4) iditoB (i) $=i-8$

C

fieldB $(i+1)=$ 'min or $h r$ ?'

fdlen $B(i+1)=10$

farowB $(i+1)=1$ incnt

fdcolB $(i+1)=55$

ficolB $(i+1)=67$

$\operatorname{mxnocB}(i+1)=1$

fitypB $(i+1)=$ ' $u$ '

ifrqdB $(i+1)=i$

C

if $(i, n e, 4)$ idito $(i+1)=i-7$

fieldB $(i+2)=$ 'Results needed by: date:'

fdlenB $(i+2)=25$ 
farowB $(i+2)=1$ inent +1

$\mathrm{facolB}(i+2)=4$

ficolB $(i+2)=31$

$\operatorname{mxnOCB}(i+2)=6$

fityp $B(i+2)=' d$ '

ifrgdB $(i+2)=i-1$

if $(i . n e .4)$ iditoB $(i+2)=i-6$

C

fieldB $(i+3)=$ 'time:'

fdlen $(i+3)=5$

fdrowB $(i+3)=$ incnt +1

fdcolB $(i+3)=41$

ficolB $(i+3)=48$

$\operatorname{m\times nOCB}(i+3)=5$

fitypB $(i+3)=$ ' $t$ '

C

if $(i \cdot n e .4)$ iditoB $(i+3)=i-5$

C

$$
\begin{aligned}
& \text { fieldB }(i+4)=\text { 'Completed:' } \\
& \text { fdlenB }(i+4)=10 \\
& \text { fdrowB }(i+4)=1 \text { incnt }+1 \\
& \text { fdcolB }(i+4)=57 \\
& \text { ficolB }(i+4)=69 \\
& \text { mxnocB }(i+4)=6 \\
& \text { fitypB }(i+4)=\text { 'd' } \\
& \text { ifrgdB }(i+4)=i+5 \\
& \text { if }(i \cdot \text { ne. } 4) \text { idito }(i+4)=i-4
\end{aligned}
$$

fieldB $(i+5)=$ 'Results report citation:'

fdlenB $(i+5)=24$

fdrowB $(i+5)=1$ incnt +2

fdcolB $(i+5)=4$

$f i \operatorname{colB}(i+5)=30$

$\operatorname{mxnOCB}(i+5)=20$

fitypB $(i+5)=$ ' $a$ '

ifrgdB $(i+5)=i+4$

if $(i$.ne. 4$)$ iditoB $(i+5)=i-3$

C

fieldB $(i+6)=$ 'Special Instructions?'

fdlenB $(i+6)=21$

fdrowB $(i+6)=1$ incnt +2

fdcolB $(i+6)=54$

$f i \operatorname{col} B(i+6)=77$

$\operatorname{mxnOCB}(i+6)=1$

fitypB $(i+6)='$ ?'

ifrqdB $(i+6)=i-1$

if $(i$.ne. 4$)$ iditoB $(i+6)=i-2$

enddo

C

fieldB $(11)='$ Strontium-89 and -90 ? $^{\prime}$

fdlenB $(11)=21$

fdrowB $(11)=8$

fdcolB $(11)=1$

ficolB $(11)=24$

mxnocB $(11)=1$

fitypB $(11)=$ '?'

C

fieldB (19) ='Total strontium?'

fdlen $B(19)=16$

fdrowB $(19)=11$

fdcolB $(19)=1$ 
ficolB $(19)=19$

$\operatorname{mxnOCB}(19)=1$

C

fitypB $(19)=$ '?'

fieldB (27)='Tritium?'

fdlenB $(27)=8$

fdrowB $(27)=14$

$\mathrm{fdcolB}(27)=1$

$f i \operatorname{colB}(27)=11$

$\operatorname{mxnocB}(27)=1$

C

$$
\text { fitypB (27) ='?' }
$$

fieldB (35) =' Other?'

fdlenB $(35)=6$

fdrowB $(35)=17$

fdcolB $(35)=1$

ficolB $(35)=9$

$\operatorname{mxnocB}(35)=1$

C

$$
\text { fitypB (35) = '?' }
$$

fieldB (36) ='Length of count:'

fdlenB $(36)=16$

fdrowB $(36)=17$

fdcolB $(36)=29$

$f i \operatorname{colB}(36)=47$

$\operatorname{mxnocB}(36)=4$

fitypB $(36)=$ ' $n$ '

C

iditoB $(36)=28$

C

fieldB (37)='min or hr?'

fdlen $B(37)=10$

fdrowB $(37)=17$

$\mathrm{fdcolB}(37)=55$

ficolB $(37)=67$

$\operatorname{mxnocB}(37)=1$

fitypB $(37)=' u$ '

ifrqdB $(37)=36$

idito $B(37)=29$

fieldB (38) = 'Nickel-63?'

fdlen $B(38)=10$

fdrowB $(38)=18$

fdcolB $(38)=4$

ficolB $(38)=16$

$\operatorname{mXnOCB}(38)=1$

fitypB $(38)=$ '?'

C

ifrgdB $(38)=35$

fieldB (39) ='Iron-55?'

fdlenB $(39)=8$

fdrowB $(39)=18$

fdcolB $(39)=21$

$f i \operatorname{colB}(39)=31$

$\operatorname{mxnOcB}(39)=1$

fitypB $(39)=$ '?'

C

ifrgaB $(39)=35$

fieldB $(40)=$ 'Sulfur-35?'

fdlen $B(40)=10$

fdrowB $(40)=18$ 
C

$$
\begin{aligned}
& \operatorname{fdcolB}(40)=36 \\
& \operatorname{ficolB}(40)=48 \\
& \operatorname{mxnocB}(40)=1 \\
& \operatorname{fitypB}(40)=' ? ' \\
& \text { ifrgdB }(40)=35
\end{aligned}
$$

fieldB $(41)=$ 'Plutonium-241?'

fdlenB $(41)=14$

fdrowB $(41)=18$

$f d \operatorname{colB}(41)=53$

$f i \operatorname{col} B(41)=69$

$\operatorname{mxnOCB}(41)=1$

fitypB $(41)=$ ' ?'

C

ifrgdB $(41)=35$

fieldB $(42)=$ 'Results needed by: date:' fdlenB $(42)=25$

fdrowB $(42)=19$

$\mathrm{fdcolB}(42)=4$

$f i \operatorname{col} B(42)=31$

$\operatorname{mxnOCB}(42)=6$

fitypB $(42)=' d$ '

ifrgdB $(42)=35$

C

$$
\text { iditoB }(42)=30
$$

fieldB $(43)=$ 'time:'

fdlenB $(43)=5$

fdrowB $(43)=19$

$\mathrm{fdcolB}(43)=41$

$f i \operatorname{col} B(43)=48$

$\operatorname{mxnocB}(43)=5$

fitypB $(43)=$ ' $t$ '

C

iditoB $(43)=31$

fieldB (44) = 'Completed:'

fdlen $B(44)=10$

fdrowB $(44)=19$

$\operatorname{facolB}(44)=57$

$\mathrm{ficolB}(44)=69$

$\operatorname{mxnocB}(44)=6$

fitypB $(44)=$ ' $d$ '

ifrgaB $(44)=45$

C

iditoB $(44)=32$

fieldB(45)='Results report citation:'

fdlen $(45)=24$

fdrowB $(45)=20$

$\mathrm{fdcolB}(45)=4$

$f i \operatorname{col} B(45)=30$

$\operatorname{mxnocB}(45)=20$

fitypB (45) =' $a$ '

ifrgdB $(45)=44$

C

$$
\text { iditoB }(45)=33
$$

fieldB $(46)=$ 'Special Instructions?'

fdlen $B(46)=21$

fdrowB $(46)=20$

$\operatorname{fdcolB}(46)=54$

ficolB $(46)=77$

$\operatorname{mxnOcB}(46)=1$ 


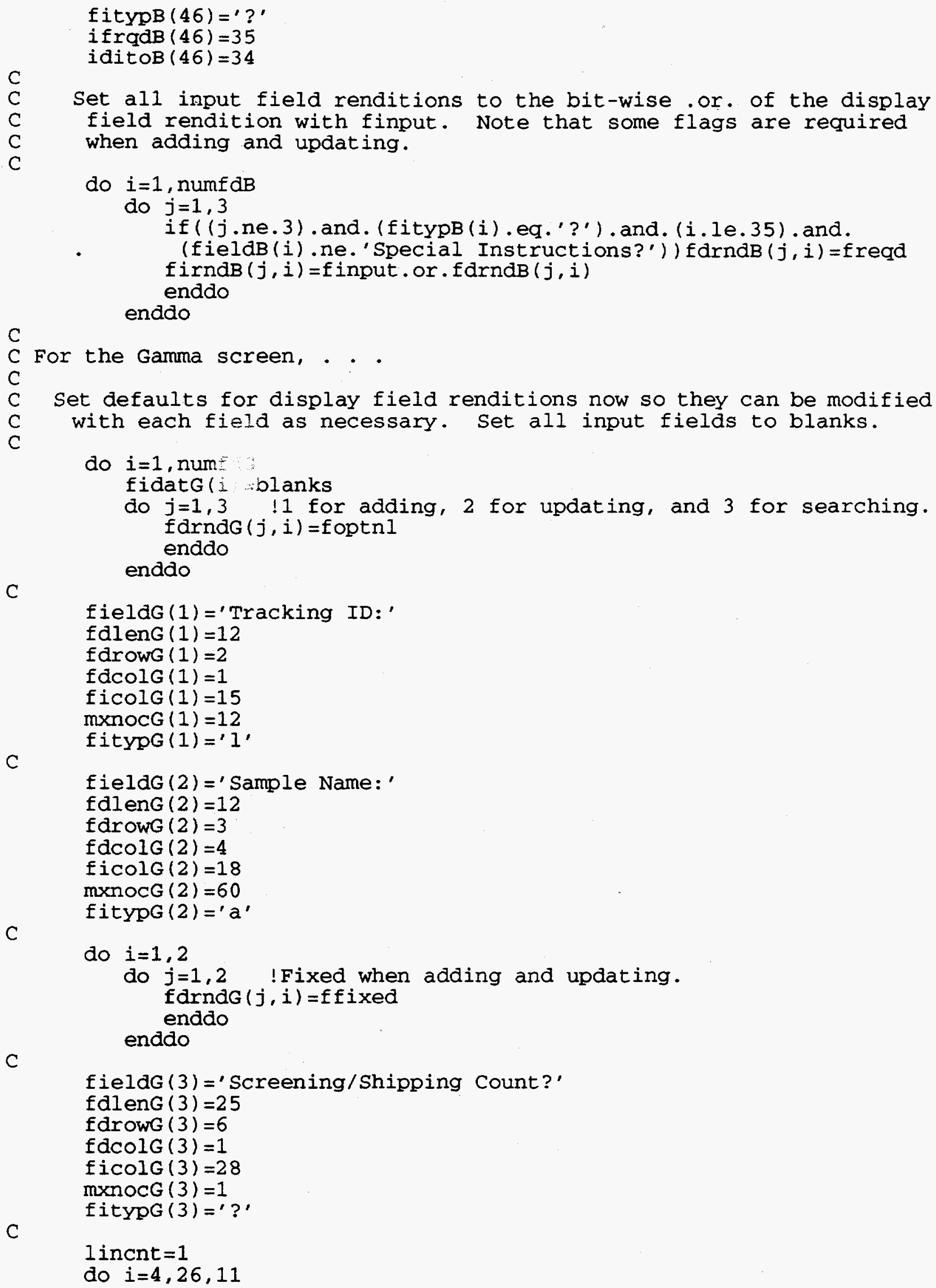


lincnt $=1$ incnt +5

fieldG(i)='Length of count:'

fdleng $(i)=16$

farowg $(i)=1$ incnt

fdcolg $(i)=37$

$f i \operatorname{colg}(i)=55$

$\operatorname{mxnocG}(i)=4$

fitypG $(i)=$ 'n'

if (i.ne.4) iditoG $(i)=i-11$

C

fieldG $(i+1)=$ 'min or $h r$ ?'

fdleng $(i+1)=10$

fdrowg $(i+1)=1$ incnt

fdcolg $(i+1)=63$

ficolg $(i+1)=75$

$\operatorname{mxnocG}(i+1)=1$

fitypg $(i+1)=$ ' $u$ '

ifrgdG $(i+1)=i$

C

if $(i . n e .4)$ iditog $(i+1)=i-10$

fieldG $(i+2)=$ 'Results needed by: date:'

fdleng $(i+2)=25$

fdrowg $(i+2)=1$ incnt +1

fdcolg $(i+2)=4$

ficolG $(i+2)=31$

$\operatorname{mxnocG}(i+2)=6$

fitypg $(i+2)=$ ' d'

ifrgdG $(i+2)=i-1$

C

if $(i . n e .4)$ iditog $(i+2)=i-9$

fieldG $(i+3)=$ 'time: '

fdleng $(i+3)=5$

fdrowg $(i+3)=1$ incnt +1

fdcolg $(i+3)=41$

ficolg $(i+3)=48$

$\operatorname{mxnOCG}(i+3)=5$

fitypg $(i+3)=' t$ '

C

if $(i$.ne. 4 ) iditog $(i+3)=i-8$

fieldG $(i+4)=$ 'Completed:'

fdleng $(i+4)=10$

fdrowg $(i+4)=1$ incnt +1

fdcolg $(i+4)=57$

ficolg $(i+4)=69$.

$\operatorname{mXnOCG}(i+4)=6$

fitypg $(i+4)=$ ' $d$ '

ifrgdG $(i+4)=i+8$

C

if $(i . n e .4$ ) iditog $(i+4)=i-7$

fieldG $(i+5)=$ 'Date counted:'

fdleng $(i+5)=13$

fdrowg $(i+5)=1$ inent +2

fdcolg $(i+5)=4$

ficolg $(i+5)=19$

$\operatorname{m\times nOCG}(i+5)=6$

fitypG $(i+5)=$ ' $d$ '

C

ifrgdG $(i+5)=i+4$

fieldG $(i+6)=$ 'RML Spectral ID:'

fdleng $(i+6)=16$ 
C

C

C

C

C

$c$

C

C

C

C

fdrowg $(i+6)=1$ incnt +2

facolg $(i+6)=29$

ficolg $(i+6)=47$

$\operatorname{mxnOCG}(i+6)=14$

fitypg $(i+6)=' 1$ '

ifrgdG $(i+6)=i+5$

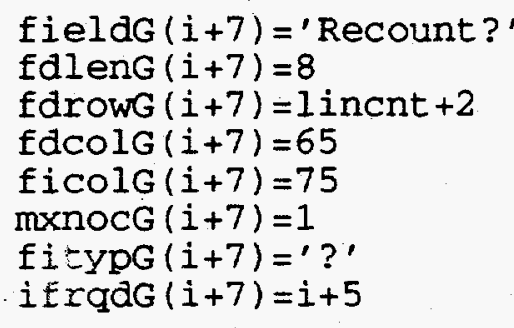

fieldG $(i+8)=$ 'Results report citation:'

fdleng $(i+8)=24$

fdrowg $(i+8)=1$ incnt +3

facolG $(i+8)=4$

ficolg $(i+8)=30$

$\operatorname{mxnocG}(i+8)=20$

fitypg $(i+8)={ }^{\prime} a^{\prime}$

ifrqdG $(i+8)=i+4$

if $(i . n e .4)$ iditog $(i+8)=i-3$

fieldG $(i+9)=$ 'Special Instructions?'

faleng $(i+9)=21$

farowg $(i+9)=1$ incnt +3

fdcolg $(i+9)=54$

ficolg $(i+9)=77$

mXnocG $(i+9)=1$

fitypg $(i+9)=$ '?'

ifrqdG $(i+9)=i-1$

if $(i . n e .4)$ iditog $(i+9)=i-2$

enddo

fieldG(14)='Full Isotopic Gamma Analysis?'

fdleng $(14)=29$

fdrowG $(14)=11$

fdcolg $(14)=1$

ficolg $(14)=32$

mXnOCG $(14)=1$

fitypg (14) ='?'

fieldG(25) ='Other Gamma Analysis?'

fdleng $(25)=21$

fdrowg $(25)=16$

fdcolg $(25)=1$

$f i \operatorname{colg}(25)=24$

$\operatorname{m\times nOCG}(25)=1$

fitypg $(25)={ }^{\prime}$ ?'

Set all input field renditions to the bit-wise or. of the display field rendition with finput. Note that some flags are required when adding and updating.

do $i=1$, numfdG

do $j=1,3$

if ( (j.ne.3) .and. (fitypg (i) .eq.'?') . and. 


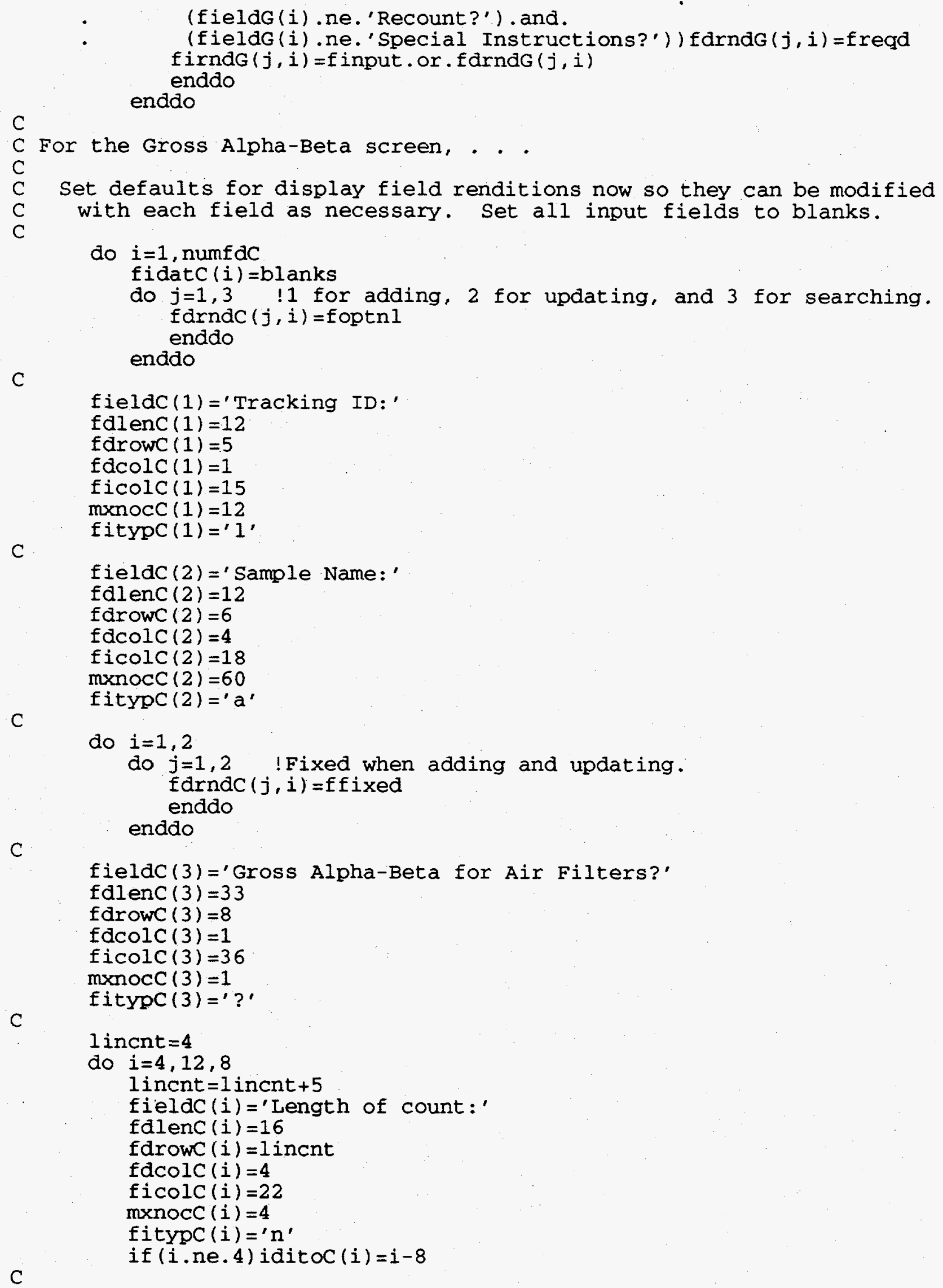


fieldc $(i+i)=$ 'min or hr?'

fdlenc $(i+1)=10$

fdrowc $(i+1)=$ lincnt

fdcolc $(i+1)=30$

ficolc $(i+1)=42$

$\operatorname{mxnocC}(i+1)=1$

fitypc $(i+1)=$ ' $u$ '

if $\operatorname{rqdC}(i+1)=i$

C if $(i$.ne.4) iditoc $(i+1)=i-7$

fieldC $(i+2)=$ 'Results needed by: date:'

fdlenc $(i+2)=25$

fdrowC $(i+2)=$ lincnt +1

$\operatorname{fdcolc}(i+2)=4$

ficolc $(i+2)=31$

$\operatorname{mxnocC}(i+2)=6$

fito $\mathrm{pC}(i+2)={ }^{\prime} \mathrm{d}^{\prime}$

if: $\quad c(i+2)=i-1$

C

í̃ :e.4) iditoc $(i+2)=i-6$

C

fieidc $(i+3)=$ 'time:'

fdlenc $(i+3)=5$

fdrow $(i+3)=1$ incnt +1

fdcolc $(i+3)=41$

ficolc $(i+3)=48$

$\operatorname{mxnocC}(i+3)=5$

fitypC $(i+3)=$ ' $t$ '

if (i.ne.4) iditoc $(i+3)=i-5$

fieldC $(i+4)=$ ' Completed:'

fdlenc $(i+4)=10$

farowC $(i+4)=$ incnt +1

fdcolc $(i+4)=57$

ficolc $(i+4)=69$

$\operatorname{mxnocC}(i+4)=6$

fitypc $(i+4)=' d$ '

ifrgdC $(i+4)=i+5$

if $(i$. ne. 4$)$ iditoc $(i+4)=i-4$

C

fieldc $(i+5)=$ 'Results report citation:'

fdlenc $(i+5)=24$

fdrowC $(i+5)=1$ incnt +2

facolc $(i+5)=4$

ficolc $(i+5)=30$

mxnocC $(i+5)=20$

fitypc $(i+5)=$ ' $a$ '

ifrqdC $(i+5)=i+4$

C

if $(i . n e .4)$ iditoc $(i+5)=i-3$

fieldc $(i+6)=$ 'Special Instructions?'

fdlenc $(i+6)=21$

fdrowC $(i+6)=1$ incnt +2

fdcolc $(i+6)=54$

ficolc $(i+6)=77$

$\operatorname{mxnocC}(i+6)=1$

fitypc $(i+6)=$ '?'

ifrgdc $(i+6)=i-1$

if $(i . n e .4)$ iditoc $(i+6)=i-2$

C enddo 
fieldC $(11)=$ 'Gross Alpha-Beta for Other Samples?'

fdlenc $(11)=35$

farowC $(11)=13$

$\mathrm{fdcolc}(11)=1$

ficolc $(11)=38$

$\operatorname{mxnocC}(11)=1$

fitypc $(11)=$ ' ?' $^{\prime}$

$\mathrm{C}$

C

C

C

C

Set all input field renditions to the bit-wise .or. of the display field rendition with finput. Note that some flags are required when adding and updating.

do $i=1$, numfdC

do $j=1,3$

if ( (j.ne. 3) .and. (fitypc (i).eq.'?') . and.

(fieldc (i).ne.'Special Instructions?') ) fdrndc $(j, i)=f r e q d$

firndC $(j, i)=f$ input.or.farndC $(j, i)$

enddo enddo

$\mathrm{C}$

C

C

C Set defaults for display field renditions now so they can be modified

C with each field as necessary. Set all input fields to blanks.

$\mathrm{C}$

do $i=1$, numfdI

fidat $I(i)=b l a n k s$

do $j=1,3$ !1 for adding, 2 for updating, and 3 for searching. fdrnd $(j, i)=$ foptn $I$ enddo

C enddo

fieldI (1)='Tracking ID:'

fdlenI $(1)=12$

farowI $(1)=2$

fdcol I $(1)=1$

ficolI $(1)=15$

$\operatorname{mxnocI}(1)=12$

fitypI $(1)=' 1$ '

C

fieldI (2) =' Sample Name:'

fdlenI $(2)=12$

fdrowI $(2)=3$

fdcol I $(2)=4$

$\mathrm{ficolI}(2)=18$

$\operatorname{mxnocI}(2)=60$

C

fitypI $(2)=$ ' $a$ '

do $i=1,2$

do $j=1,2 \quad$ !Fixed when adding and updating. fdrndI $(j, i)=$ ffixed enddo

enddo

C

do $i=3,31,2$

fieldI $(i)=$ 'From'

fdlenI $(i)=4$

farowI $(i)=3+(i+1) / 2$

$f d \operatorname{col} I(i)=4$

$f i \operatorname{col} I(i)=9$ 
C

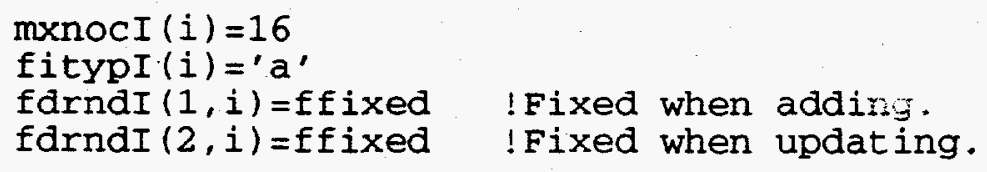

fieldI $(i+1)=$ ' $^{\prime}$

fdlenI $(i+1)=1$

fdrowI $(i+1)=3+(i+1) / 2$

fdcolI $(i+1)=25$

$f i \operatorname{col} I(i+1)=28$

$\operatorname{mxnocI}(i+1)=50$

fitypI $(i+1)=' a$ '

enddo

C

C Set all input field renditions to the bit-wise .or. of the display C field rendition with finput.

C

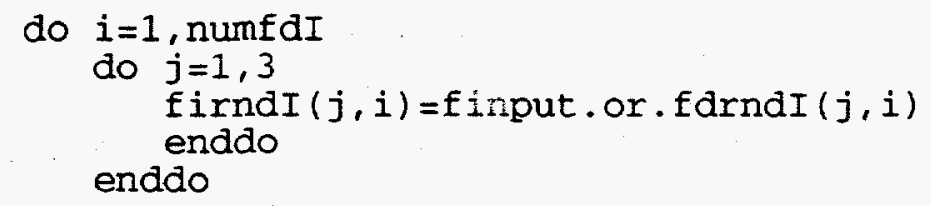

C

C For the Possessor screen,. . .

C Set defaults for display field renditions now so they can be modified

C with each field as necessary. Set all input fields to blanks.

C

do $i=1$, numfdT

fidat $(i)=$ blanks

do $j=1,3$ !1 for adding, 2 for updating, and 3 for searching. fdrndT $(j, i)=$ foptn 1

enddo

C

fieldT(1)='Tracking ID:'

fdlenT $(1)=12$

fdrowT $(1)=3$

$\mathrm{fdcolT}(1)=1$

$\mathrm{ficolT}(1)=15$

$\operatorname{mXnOCT}(1)=12$

C

fitypt $(1)=' 1^{\prime}$

fieldT $(2)=$ 'Sample Name:'

fdlenT $(2)=12$

fdrow $T(2)=4$

fdcolt $(2)=4$

ficolT $(2)=18$

mXnocT $(2)=60$

C

$$
\text { fitypt }(2)=' a^{\prime}
$$

do $i=1,2$

do $j=1,2 \quad$ ! Fixed when adding and updating. fdrndT $(j, i)=f f i x e d$ enddo

C enddo

lincnt $=1$

do $i=3,17,7$

lincnt $=1$ incnt +5 
fieldT $(i)=$ ' Possessor

fdlent $(i)=11$

farow $(i)=1$ incnt

$\operatorname{fdcolT}(i)=1$

$f i \operatorname{colT}(i)=13$

$\operatorname{mxnOCT}(i)=3$

fitypT $(i)=$ 'n'

fdrndT $(1, i)=$ ffixed $!$ Fixed when adding and

C

fdrndT $(2, i)=$ ffixed ! updating.

C

fieldT $(i+1)={ }^{\prime}: '$

fdlenT $(i+1)=1$

farow $(i+1)=1$ incnt

$\mathrm{fdcolT}(i+1)=16$

ficolT $(i+1)=22$

$\operatorname{mxnocT}(i+1)=25$

fitypT $(i+1)=' a$ '

if (i.eq. 3 ) then

fdrndT $(1,(i+1))=$ ffixed

fdrndT $(2,(i+1))=$ ffixed

else

ifrqdT $(i+1)=i-1$

endif

fieldT $(i+2)=$ 'Phone Number: '

fdient $(i+2)=13$

fdrowT $(i+2)=$ linicnt

$\operatorname{fdcolT}(i+2)=51$

ficolT $(i+2)=66$

$\operatorname{mxnocT}(i+2)=12$

fitypT $(i+2)=$ ' $a$ '

if (i.eq. 3 ) then

fdrndT $(1,(i+2))=$ ffixed fdrndT $(2,(i+2))=f f i x e d$

!Fixed when adding and else

endif

ifrqdT $(i+2)=i+1$

C

fieldT $(i+3)=$ 'Address:'

fdlenT $(i+3)=8$

fdrow $T(i+3)=1$ incnt +1

fdcolt $(i+3)=4$

ficolT $(i+3)=14$

$\operatorname{mxnocT}(i+3)=60$

fitypT $(i+3)=a^{\prime}$

if (i.eq. 3$)$ then

fdrndT $(1,(i+3))=$ ffixed

fdrndT $(2,(i+3))=f f i x e d$

! updating.

!Fixed when adding and ! updating. else

ifradT $(i+3)=i+1$

endif

C

fieldT $(i+4)=$ 'Reason for possession:'

fdlenT $(i+4)=22$

fdrow $T(i+4)=1$ incnt +2

fdcolT $(i+4)=4$

ficolT $(i+4)=28$

mxnoct $(i+4)=50$

fitypT $(i+4)=$ ' $a$ '

if (i.eq. 3 ) then

!Fixed when adding and ! updating. 
C

C

C

C

C

C

C

C

C

C.

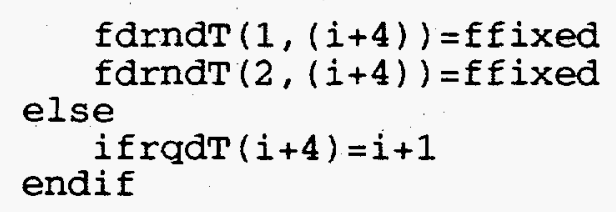

fdrndT $(1,(i+4))=$ ffixed

endif

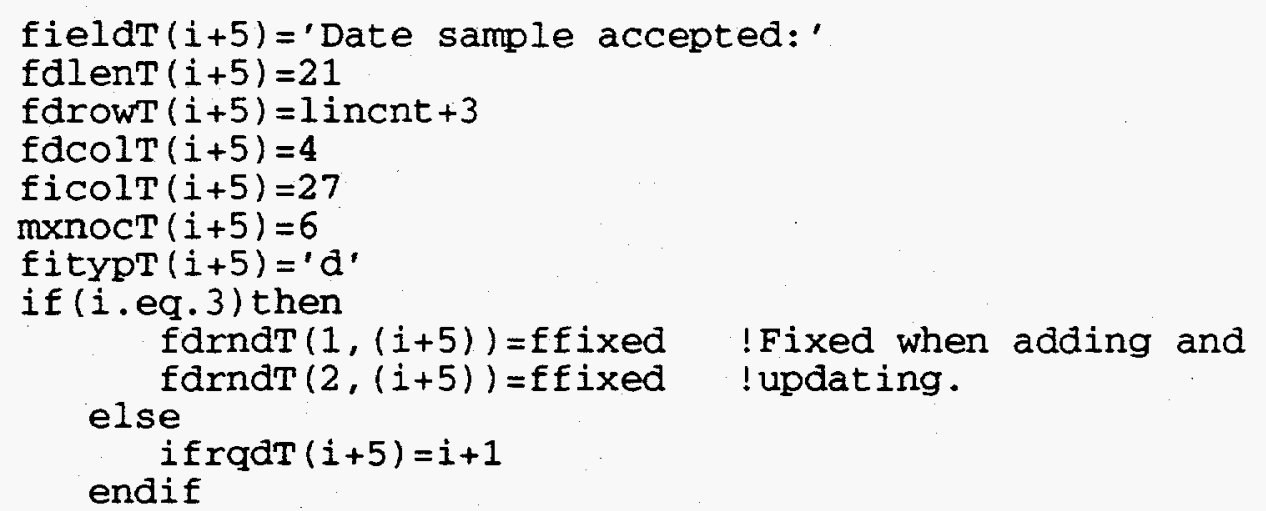

!Fixed when adding and ! updating.

if(i.ne.3) iditoT $(i+5)=i-1$. To las te sample relinquished.

fieldT $(i+6)=$ 'Date sample relinquishe

fdlent $(i+6)=25$

fdrow $(i+6)=$ lincnt +3

fdcolT $(i+6)=37$

$f i \operatorname{colT}(i+6)=64$

$\operatorname{mxnocT}(i+6)=6$

fitypT $(i+6)=$ 'd'

enddo

Set all input field renditions to the bit-wise .or. of the display field rendition with finput.

do $i=1$, numfdT

do $j=1,3$

firndT $(j, i)=f i n p u t . o r . f d r n d T(j, i)$

enddo enddo

Possessor name list data (set up to ease adding and removing names - note that the current limit is fifteen names).

numfdN=1

fieldN $(1$, numfdN) ='J. L. DOHERTY (JODIE)'

fdlenN $(1$, numfdN $)=21$

fieldN $(2$, numfdN $)=' 6-6573^{\prime}$

fdlenN $(2$, numfdN $)=6$

fieldN (3, numfdN) ='RML, MS 7111; TRA-604, ROOM 123'

fdlenN $(3$, numfdN $)=31$

fieldN $(4$, numfdN $)=$ 'GAMMA SPECTRUM ACQUISITION'

fdlenN $(4$, numfdN $)=26$

numfdN=numfdN +1

fieldN $(1$, numfdN $)=' T$. J. HANEY (TOM)'

fdlenN $(1$, numfdN $)=17$

fieldN $(2$, numfdN $)=' 6-4158^{\prime}$

fdlenN $(2$, numfdN $)=6$

fieldN (3, numfdN)='RML, MS 7111; TRA-604, ROOM 126'

fdlenN $(3$, numfdN $)=31$

fieldN $(4$, numfdN) ='SAMPLE ROUTING COORDINATION' 
ก

$\Omega$

$\Omega$

ก

$\Omega$

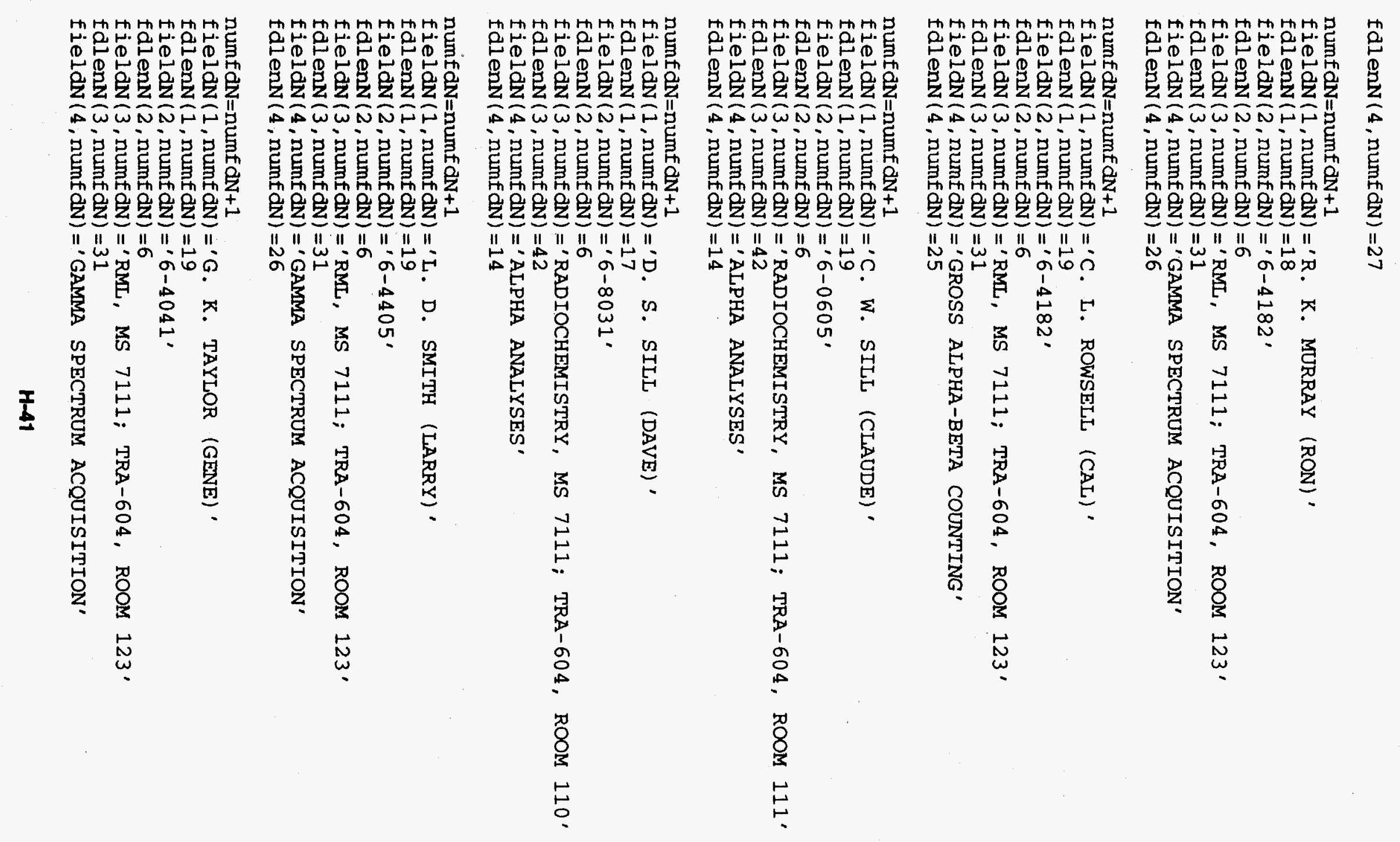


C

falenN $(4$, numfdN $)=26$

numfdN $=$ numf $d N+1$

fieldN (1, numfdN) ='L. A. WEINRICH (LOU)'

fdlenN $(1, \operatorname{numfdN})=20$

fieldN $(2$, numfdN $)=' 6-4404$ '

fdlenN $(2$, numfdN $)=6$

fieldN (3, numfdN)='RADIOCHEMISTRY, MS 7111; TRA-661, ROOM 129/130'

fdlenN $(3$, numfdN $)=46$

fieldN $(4$, numfdN) = 'BETA ANALYSES'

C

fdlenN $(4$, numfdN $)=13$

numfdN $=$ numfdN +1

fieldN (1, numfdN) ='R. P. WELLS (RICH)'

fdlenN $(1$, numfdN $)=18$

fieldN $(2$, numfdN $)=' 6-7870^{\prime}$

fdlenN $(2, \operatorname{numfdN})=6$

fieldN (3, numfdN) ='RADIOCHEMISTRY, MS 7111; TRA-661, ROOM 129/130'

fdlenN $(3$, numfdN $)=46$

fieldN $(4$, numfdN $)=$ 'BETA ANALYSES'

C

fdlenN $(4$, numfdN $)=13$

do $i=1$, numfdN

farown $(i)=i+1$

fdColN $(i)=2$

ficolN $(i)=25$

enddo 


\title{
I. DISPLY - FORTRAN ROUTINE FOR DISPLAYING DATA INPUT SCREENS USING VMS SCREEN MANAGEMENT SYSTEM ROUTINES
}

\author{
subroutine disply (field, fdatin, fldlen, fldrow, fldcol, fincol, maxnoc, \\ - fldrnd, finrnd, numfld, indaus, fintyp, ifnulf, ifregd, ifmodi, iditto, \\ - ifclst, ibegin, subttl) \\ C \\ C Subroutine for displaying data entry fields. \\ C Component of SAMPLE_TRACKING Version 1 completed June 6, 1991 by D. A. Femec. \\ C Screen design based on E. Wayne Killian's ADD_SAMPLE routine of $3 / 21 / 90$. \\ C \\ C Declaration statements. \\ $\mathrm{C}$ \\ include '(\$smgdef)' !SMG Definitions. \\ include '(\$smgmsg)' !SMG Condition values. \\ include '(\$smgtrmptr)' ISMG Terminal and printer definitions. \\ include '( $\$$ trmdef)' \\ include 'gap\$src:vterm.inc' \\ integer*4 termod, ipaste, mvideo, 1video, hvideo, keybrd, !Pasteboard values. \\ - indaus, !Add/Update/Search indicator. \\ - ibegin, tThe field on which to begin the cursor. \\ - iwhich !Which custodian has been selected from CHOOSE. \\ logical ifcont. IFlag whether to continue in display loops. \\ - ifclst !Flag whether custodian name list applies. \\ common /paste_board/ termod, ipaste, mvideo, Ivideo, hvideo, keybrd \\ integer $4^{4}$ ferror, ffixed, finput, foptnl, freqd \\ common /fldscr/ ferror, ff ixed, finput, foptnl, freqd \\ character subtt $1^{*}(*)$ !Subtitle for display. \\ character $* 80$ field (*), fdatin $(*)$, findat, blanks \\ character ${ }^{*} 1$ spaces $(80) / 80^{*}, \%$, fintyp $(*)$ \\ integer 4 fldlen(*), fldrow (*), fldcol (*), fincol (*), maxnoc (*), \\ - Eldrnd $(3, *)$, finrnd $(3, *)$, !Field renditions (add/edit/search). \\ - numfld !The number of fields. \\ integer $* 2$ daymnt $(12) / 31,28,31,30,31,30,31,31,30,31,30,31 /$, \\ - ifnulf $\left(^{*}\right)$, !Null indicator ( -1 if null, 0 otherwise). \\ - ifreqd(*), !Points to if-required dependent field (except for search). \\ - iditto $(*)$ ! Points to allowed field for dittoing. \\ logical ifmodi(*) ! Have any of the fields been modified? \\ equivalence (blanks (1:1), spaces (1)) \\ $c$ \\ C \\ Custodian name list field descriptors and sizes. \\ character $* 80$ fieldN $(4,15)$ \\ integer 4 fdlenN $(4,15), \operatorname{fdrown}(15), f d c o l N(15), f i c o l N(15)$, \\ - numfdN !The number of fields. \\ common /lsdatN/ fieldN, fdlenN, fdrown, fdcolN, ficolN, numfdN \\ C \\ $C$ Beginning of the subroutine. \\ C Renew the keyboard. \\ C \\ call smg\$delete_virtual_keyboard (keybrd) \\ call smg\$create_virtual_keyboard(keybrd) \\ $C$ \\ C Display the subtitle if there is one. \\ C. \\ if (len (subtt 1) . ne.0) then \\ if(subtt1.ne.blanks(1:1en(subtt1))) then If there is a subtitle.. \\ cal1 smg\$put_chars (mvideo, subtt 1,2,(78-1en(subtt1)), freqd) \\ endif \\ endif
}




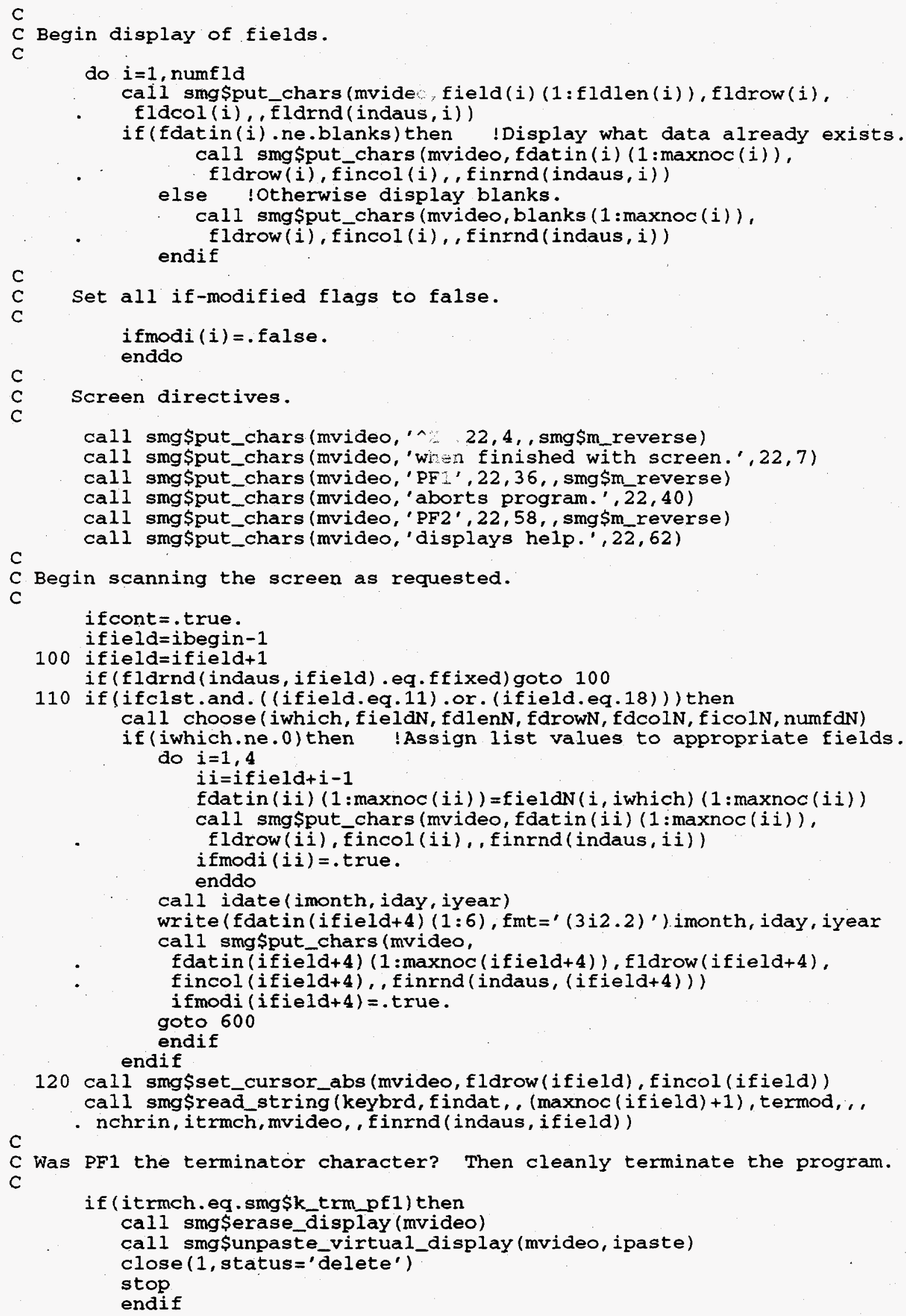


C C Was "Insert Here" the terminator character? Then ditto entry if possible. C

if (itrmch.eq.smg\$k_trm_insert_here) then if (iditto (ifield).ne.0) then fdatin (ifield)=fdatin(iditto(ifield)) !Ditto the data. call smgsput_chars (mvideo, !Write the dittoed data.

- fdatin(ifield) (1:maxnoc (ifield)), fldrow(ifield).

- . fincol(ifield), ,finrnd (indaus, ifield)) goto 400 ! Assume that ditto data is correct since original is. endif endif

C C Was PF2 or Help the terminator character? Then display keypad.

C

if ( (itrmch.eq.smg\$k_trm_help).or. (itrmch.eq.smg\$k_trm_pf2)) then call keypad goto 120 !Go back to accept new input from current field. endif

C C Check data input against requirements.

C 300 if (nchrin.gt.maxnoc (ifield)) then call smg\$erase_chars (mvideo, nchrin, fldrow(ifield), fincol (ifield)) goto 500 endif

if (nchrin.ne.0)then !So as to save entries. ifmodi (ifield) =.true.

if (nchrin.1t.maxnoc(ifield)) ! Pad each entry with blanks. findat ( (nchrin+1): maxnoc (ifield)) =blanks (1:(maxnoc (ifield) nchrin))

if (fdatin(ifield).ne.blanks) then !Rewrite the entry.

call smg\$erase_chars (mvideo, (maxnoc (ifield) +1), flarow (ifield), fincol (ifield))

call smg\$put_chars (mvideo, findat (1:maxnoc (ifield)), fldrow(ifield), fincol(ifield), , finrnd(indaus, ifield)) endif

fdatin (ifield) =findat $(1:$ nchrin) //blanks (1:(80-nchrin) ) ! Add blanks. if (fintyp (ifield) .eq.' $a$ ') goto 400 ! Free entry field.

if (fintyp (ifield).eq.' $d$ ') then ! Dates between 1/1/1990-12/31/2079. if (nchrin.ne.6) goto 500 !Must have 6 characters. read (findat $(5: 6)$, fmt $={ }^{\prime}(i 2)^{\prime}$, err $=500$, end $=500$ ) iyear if ( (iyear. It .0) .or.

( (iyear.ge.80) and. (iyear.le.89)) ) goto 500

if (mod (iyear, 4 ) .eq. 0 ) then else

daymnt (2) $=29 \quad ! 29$ days in February in a leap year. endif

daymnt $(2)=28 \quad ! 28$ days in February in a non-leap year.

read (findat $(1: 2)$, fmt $=$ ' (i2) ' , err $=500$, end $=500$ ) imonth

if ((imonth.1t.1).or. (imonth.gt.12)) goto 500

read ( $\operatorname{findat}(3: 4)$, fmt $=$ ' (i2)' , err $=500$, end $=500$ ) iday

if ( (iday. It.1) .or. (iday.gt. daymnt (imonth))) goto 500

goto 400 endif

if (fintyp(ifield).eq.' 1 ') then !Required field length. if (nchrin.eq.maxnoc (ifield)) goto 400 goto 500 endif

if(fintyp(ifield).eq.' $n$ ') then !Number, real or integer. ndecm $1=0$

do $350 \quad i=1$, nchrin

inumbr $=i \operatorname{char}(f \operatorname{indat}(i: i)$ )

if ((inumbr.ge.48) and. (inumbr.le.57)) goto 350 !Digits okay. 


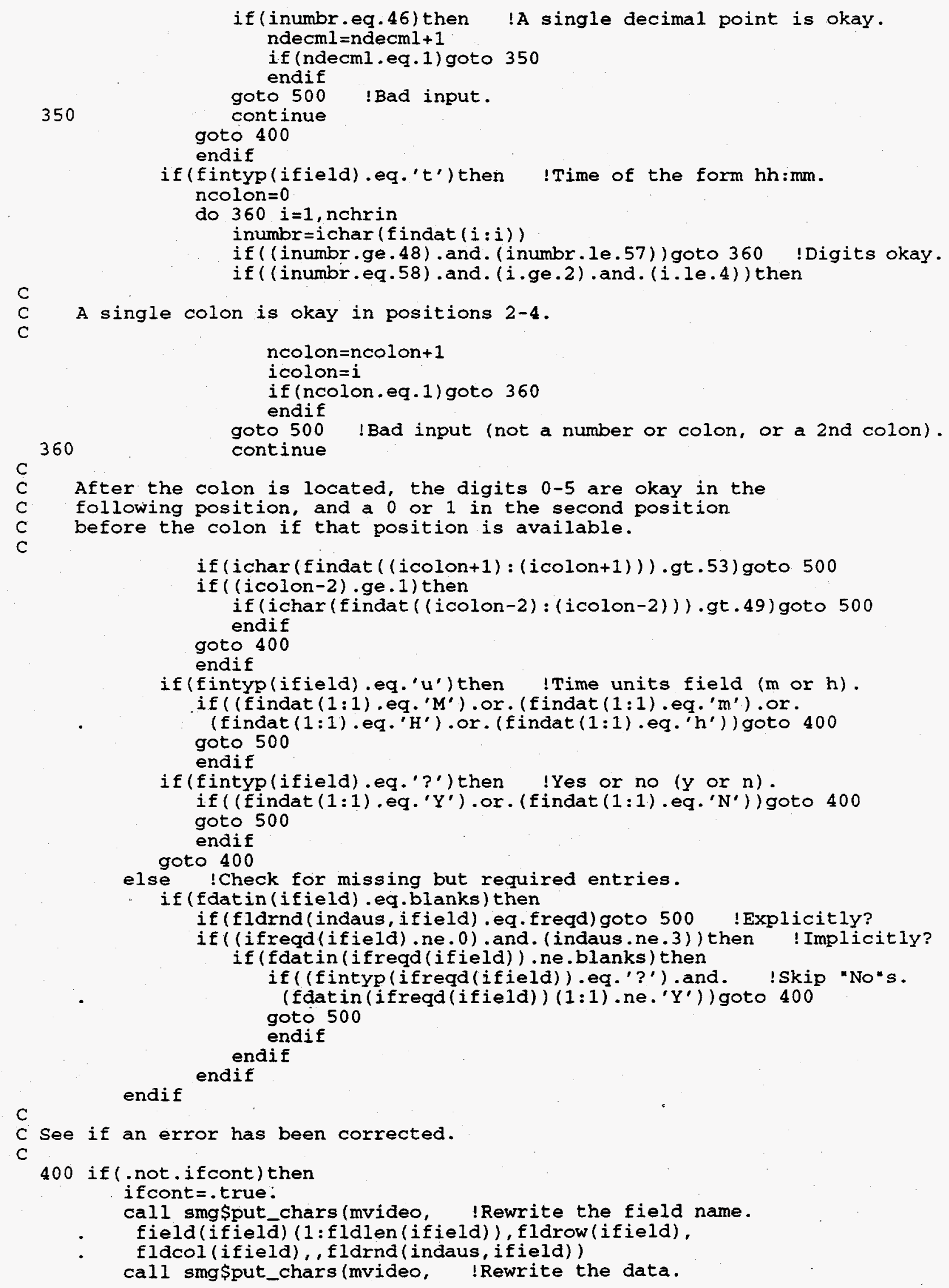




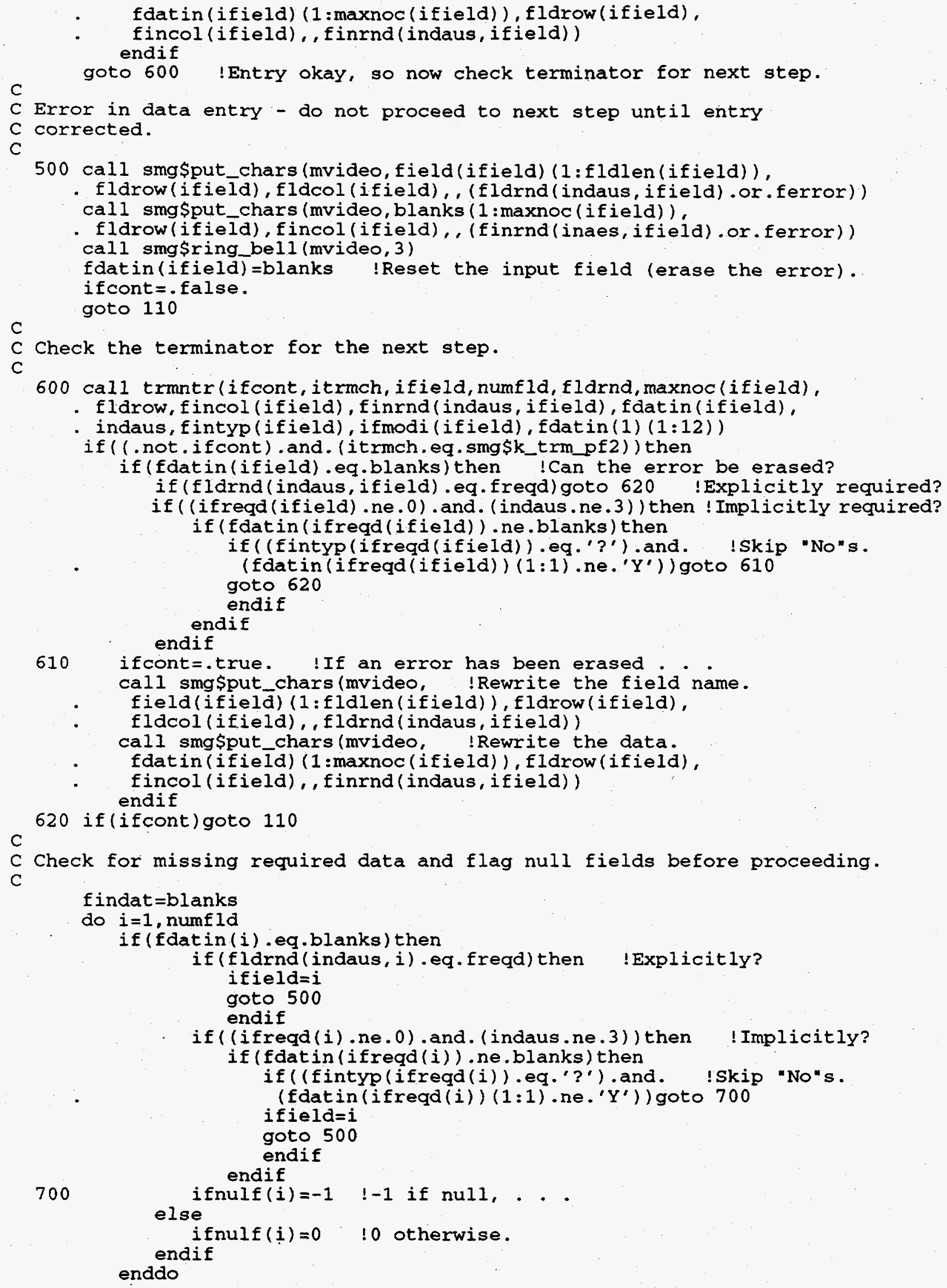


C

C Renew the keyboard.

C

call smg\$delete_virtual_keyboard (keybrd)

call smg\$create_virtual_keyboard(keybrd)

C return

$C$ End of the subroutine DISPLY.

C

end 


\title{
J. TRMNTR - FORTRAN ROUTINE FOR INTERPRETING TERMINATOR CODES FROM VMS SCREEN MANAGEMENT SYSTEM ROUTINE CALLS
}

\author{
subroutine trmntr(ifcont, itrmch, ifield, numfld, fldrnd, maxnoc, \\ C \\ fdsrow, fincol, finrnd, findat, indaus, fintyp, ifmodi, smplid) \\ C Subroutine to act on "terminators." \\ C Component of SAMPLE_TRACKING Version 1 completed June 6, 1991 by D. A. Femec. \\ C \\ C Remember to skip "fixed" fields, i.e. those fields which cannot be modified. \\ C \\ C Declaration statements. \\ C \\ include '(\$smgdef)' !SMG Definitions. \\ include' (\$smgmsg)' !SMG Condition values. \\ include '(\$smgtrmptr)' !SMG Terminal and printer definitions. \\ include ' (\$trmdef)' \\ include 'gap\$src:vterm.inc' \\ integer* 4 termod, ipaste, mvideo, Ivideo, hvideo, disply, ! Pasteboard values. \\ - indaus !Add/Update/Search indicator. \\ logical ifcont, !Flag whether to continue in display loops. \\ - ifskip !Flag whether to skip to next required. \\ common/paste_board/ termod, ipaste, mvideo, lvideo, hvideo, disply \\ character* 80 findat, blanks \\ character*12 smplid \\ character $* 1$ spaces $(80) / 80 *$ ' '/, fintyp \\ integer* 4 fldrnd(3*), fdsrow (*), fincol, finrnd \\ logical ifmodi, !Has the field been modified (here, erased)? \\ - iffrwd/.true./ ! Is the currently selected direction forward? \\ equivalence (blanks $(1: 1)$, spaces (1)) \\ integer*4 ferror, ffixed, finput; foptnl, freqd !Display field renditions. \\ common /fldscr/ ferror, ffixed, finput, foptn1, freqd \\ C \\ C Beginning of the subroutine. \\ C \\ ifcont=.true. !Set ifcont to true as default. Leave ifmodi as it is. \\ ifskip = (fldrnd (indaus, ifield) .eq. freqd) .and. (fintyp.eq. '?') .and. \\ $\mathrm{C}$ \\ . (findat $(1: 1)$.eq.' $N$ ') \\ C Advance? Then set direction as forward, but do not move. \\ C \\ if (itrmch.eq.smg\$k_trm_kp4) then \\ iffrwd=.true. \\ return \\ endif \\ C \\ C Backup? Then set direction as reverse, but do not move. \\ C \\ if (itrmch . eq.smg\$k_trm_kp5) then \\ if frwd=. false. \\ return \\ C \\ endif \\ C Return, Enter, right arrow, or horizontal tab? Advancing and \\ C Keypad 1 or 3 ? Then move to the next field. \\ C \\ if ( (itrmch.eq.smg\$k_trm_cr) .or. (itrmch.eq.smg\$k_trm_enter).or. \\ . (itrmch.eq.smg\$k_trm_right).or. (itrmch.eq.smg\$k_trm_ht).or. \\ - (( (itrmch.eq.smg\$k_trm_kp1) .or. (itrmch.eq.smg\$k_trm_kp3)) . and. \\ - iffrwd) ) then \\ 100 ifield=ifield+1
}


if (ifield.gt.numfld) then

if ield $=1$

ifskip=.false. !ifskip is no longer meaningful. endif

if(fldrnd(indaus, ifield).eq.ffixed)goto 100 !skip if fixed.

C
C

If the starting field is required, needs a yes or no answer, and was answered no, then skip to the next required field.

if ((ifskip) .and. (fldrnd (indaus, ifield) .ne.fregd)) goto 100

return

$C$ endif

$C$ Do or $\wedge \mathrm{Z}$ ? Then data input has ended for this screen and the

C display loop should not be continued.

c

if ( (itrmch.eq.smg\$k_trm_do).or. (itrmch.eq.smg\$k_trm_ctrlz)) then

ifcont $=$.false.

return

$C$ endif

C PF1? Then abort execution of the program.

C

if (itrmch.eq.smg\$k_trm_pf1) then

call smg\$erase_display (hvideo) !Help display.

call smg\$npaste_virtual_display (hvideo, ipaste)

call smg\$erase_display(1video) !"Choose" display.

call smg\$unpaste_virtual_display (Ivideo, ipaste)

call smgSerase_display (mvideo) !Main display.

call smg\$unpaste_virtual_display (mvideo, ipaste)

close (1, status='delete')

stop

C endif

C PF2? Then display keypad.

C

if ( (itrmch.eq.smg\$k_trm_help).or. (itrmch.eq.smg\$k_trm_pf2)) then call keypad

return

endif

C

C PF4, Remove, or Keypad minus, 6, or comma? Then erase the field.

C

if ( (itrmch.eq.smg\$k_trm_pf4).or. (itrmch.eq.smg\$k_trm_minus).or.

- (itrmch.eq.smg\$k_trm_comma).or. (itrmch.eq.smg\$k_trm_kp6).or.

- (itrmch.eq.smg\$k_trm_remove)) then

call smg\$erase_chars (mvideo, maxnoc, fdsrow(ifield), fincol)

call smg\$put_chars (mvideo,blanks ( 1 : maxnoc), fdsrow (ifield), fincol, , finrnd)

findat $=$ blanks

ifmodi=.true. !Erasing a field changes it.

return

endif

C

C Left arrow? Backing up and Keypad 1 or 3? Then move to the previous field.

C

if ( (itrmch.eq.smg\$k_trm_left).or. (( (itrmch.eq.smg\$k_trm_kp1).or.

200 (itrmch.eq.smg\$k

if (ifield.1t.1) ifield=numfld

if (fldrnd(indaus, ifield).eq.ffixed)goto 200 !skip if fixed.

return

C endif 


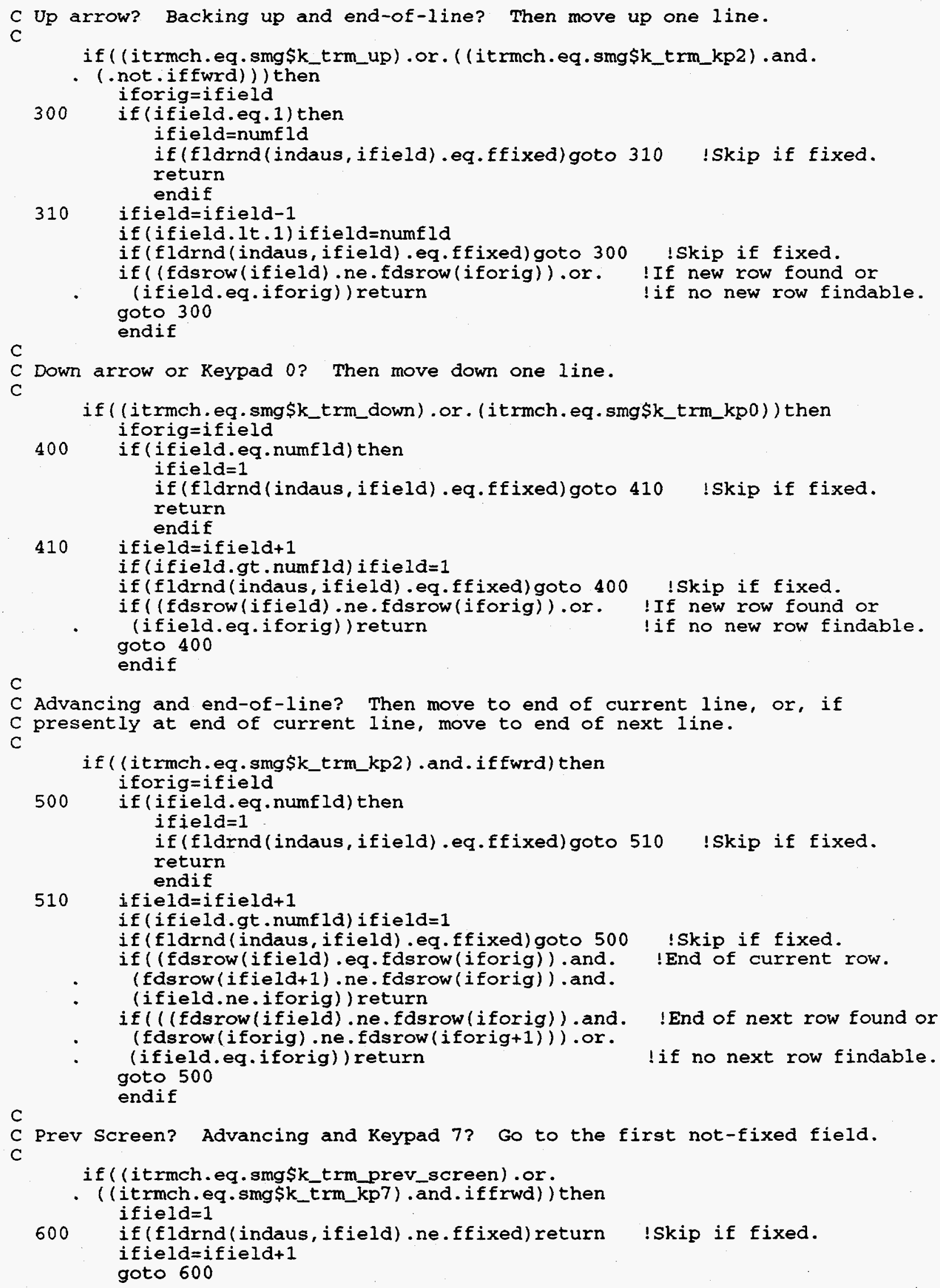




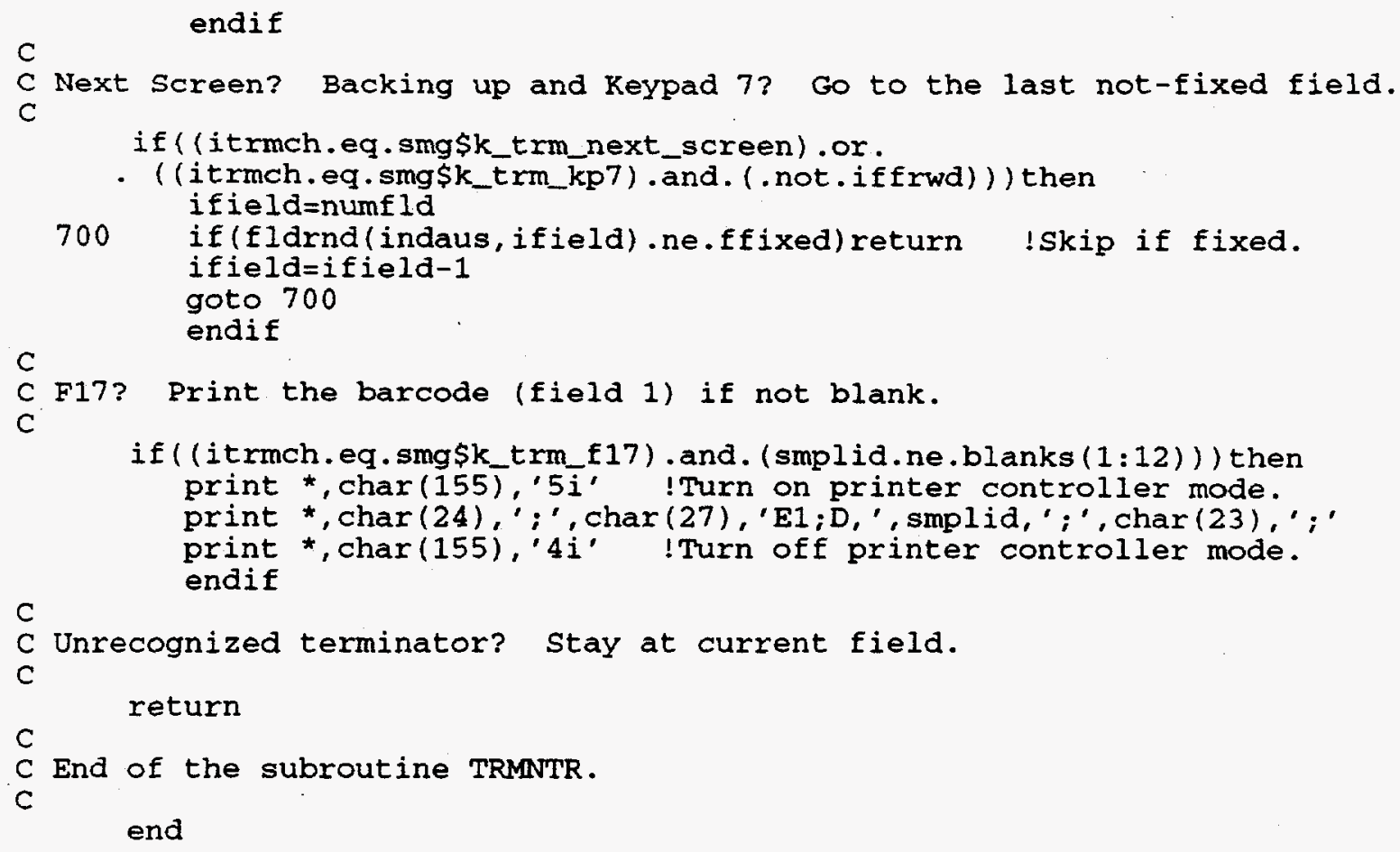




\section{K. CHOOSE - FORTRAN ROUTINE FOR PRESENTING A LIST OF CHOICES USING VMS SCREEN MANAGEMENT SYSTEM ROUTINES}

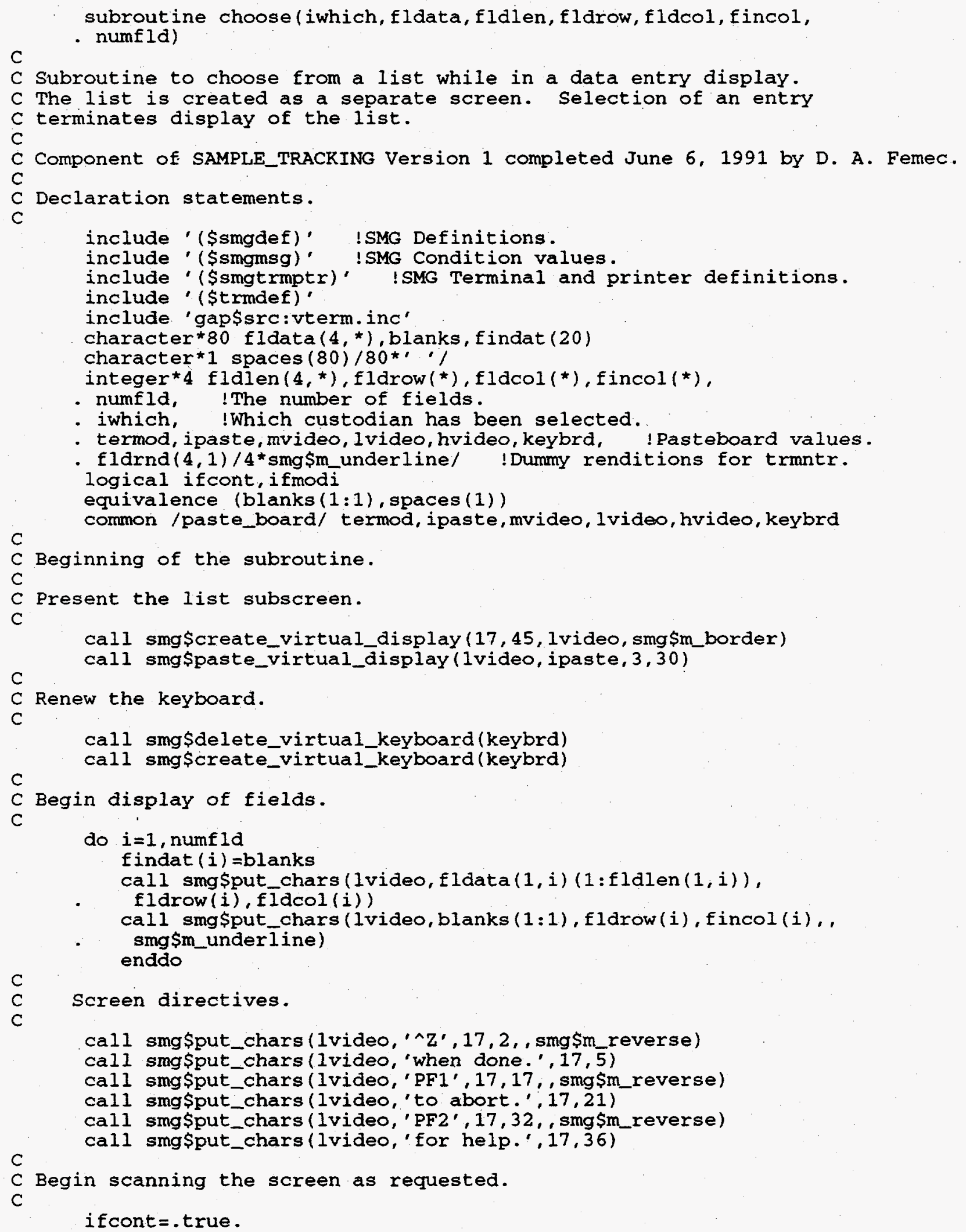




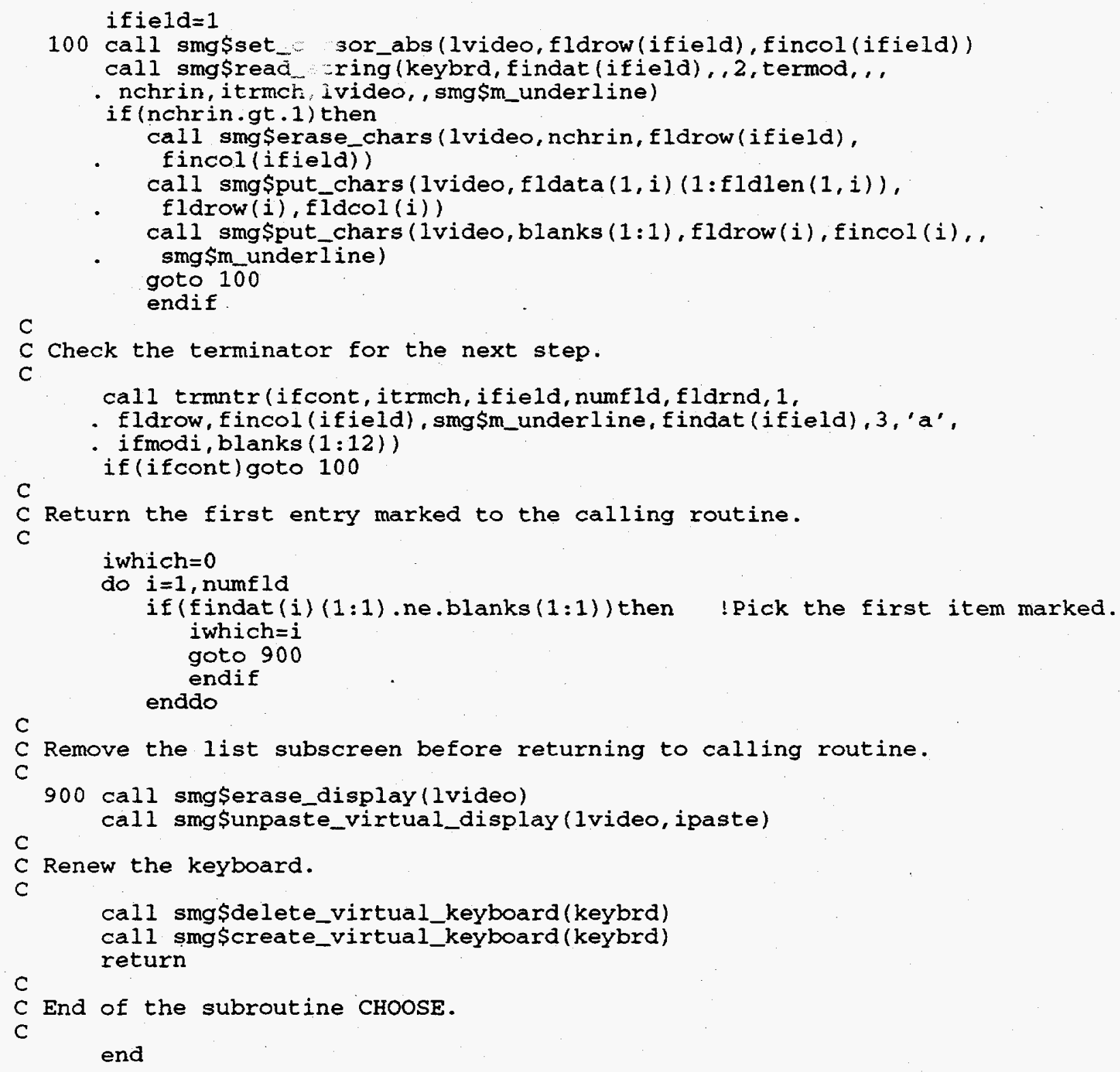

ifield $=1$ 


\section{KEYPAD - FORTRAN ROUTINE FOR DISPLAYING KEYPAD MAPPING USING VMS SCREEN MANAGEMENT SYSTEM ROUTINE}

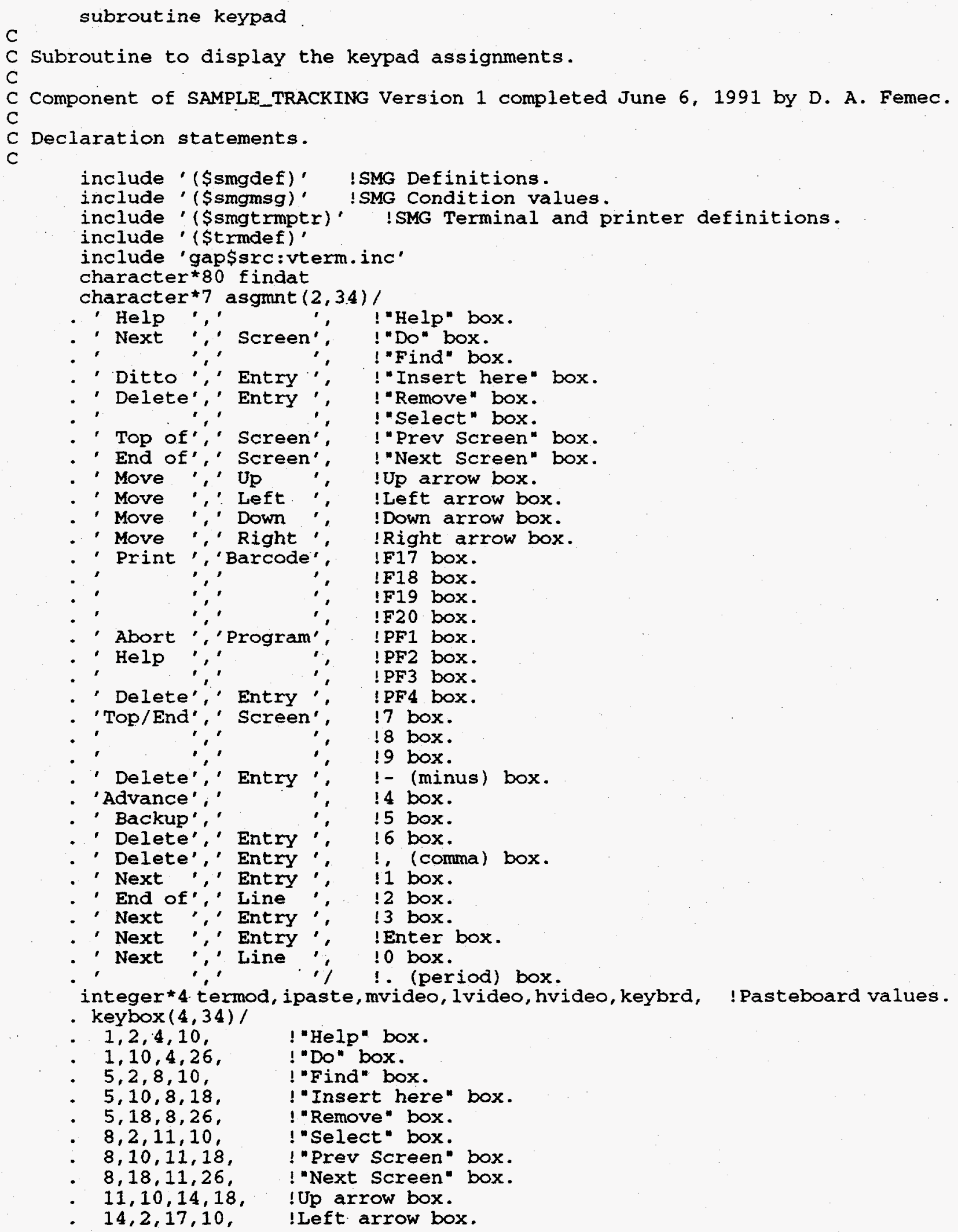




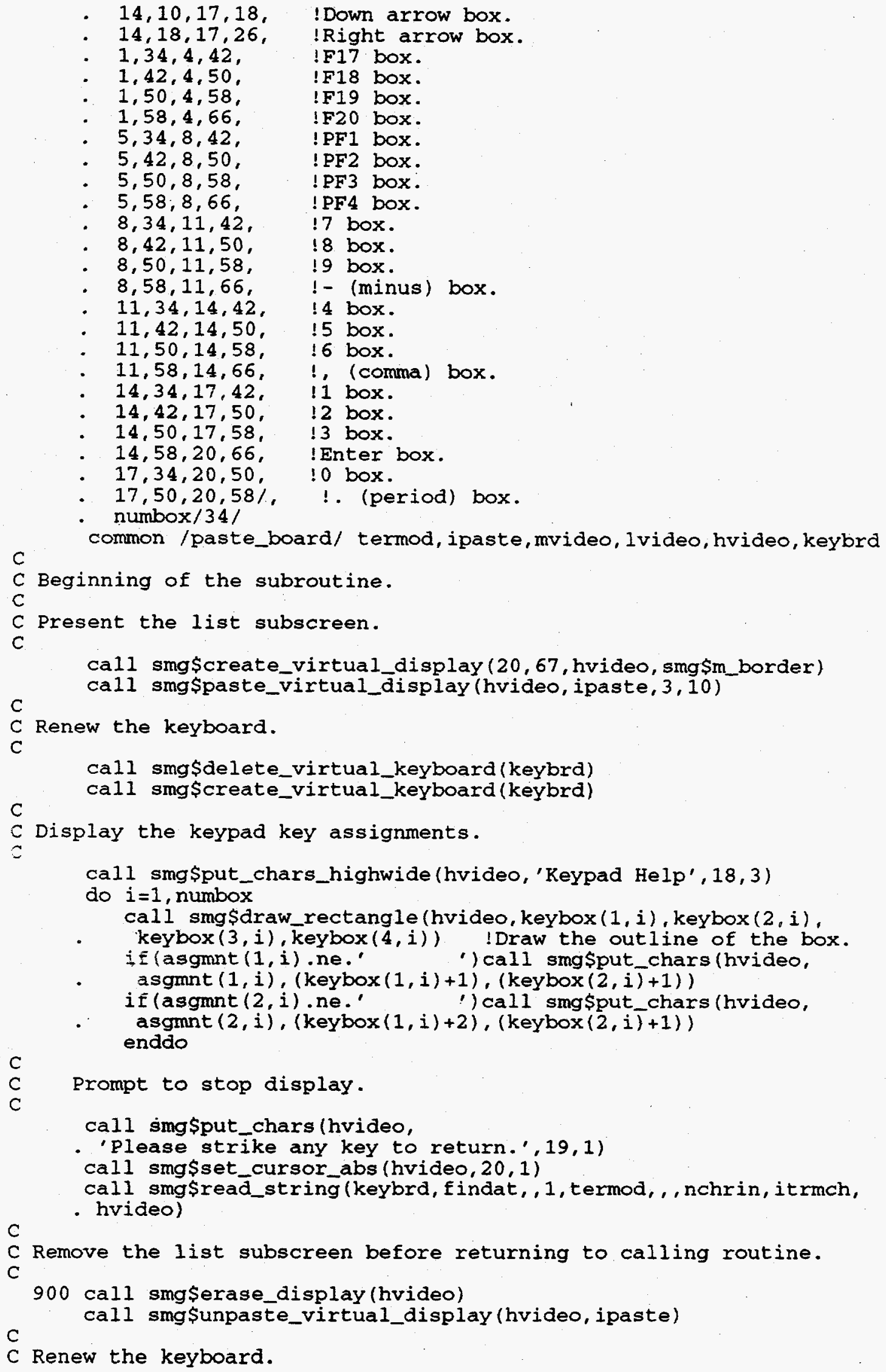


C

call smg\$delete_virtual_keyboard(keybrd)

call smg\$create_virtual_keyboard(keybrd)

C return

C End of the subroutine KEYPAD.

end 


\section{RVALUE - FORTRAN ROUTINE FOR CHARACTER STRING-TO- NUMERICAL VALUE CONVERSIONS}

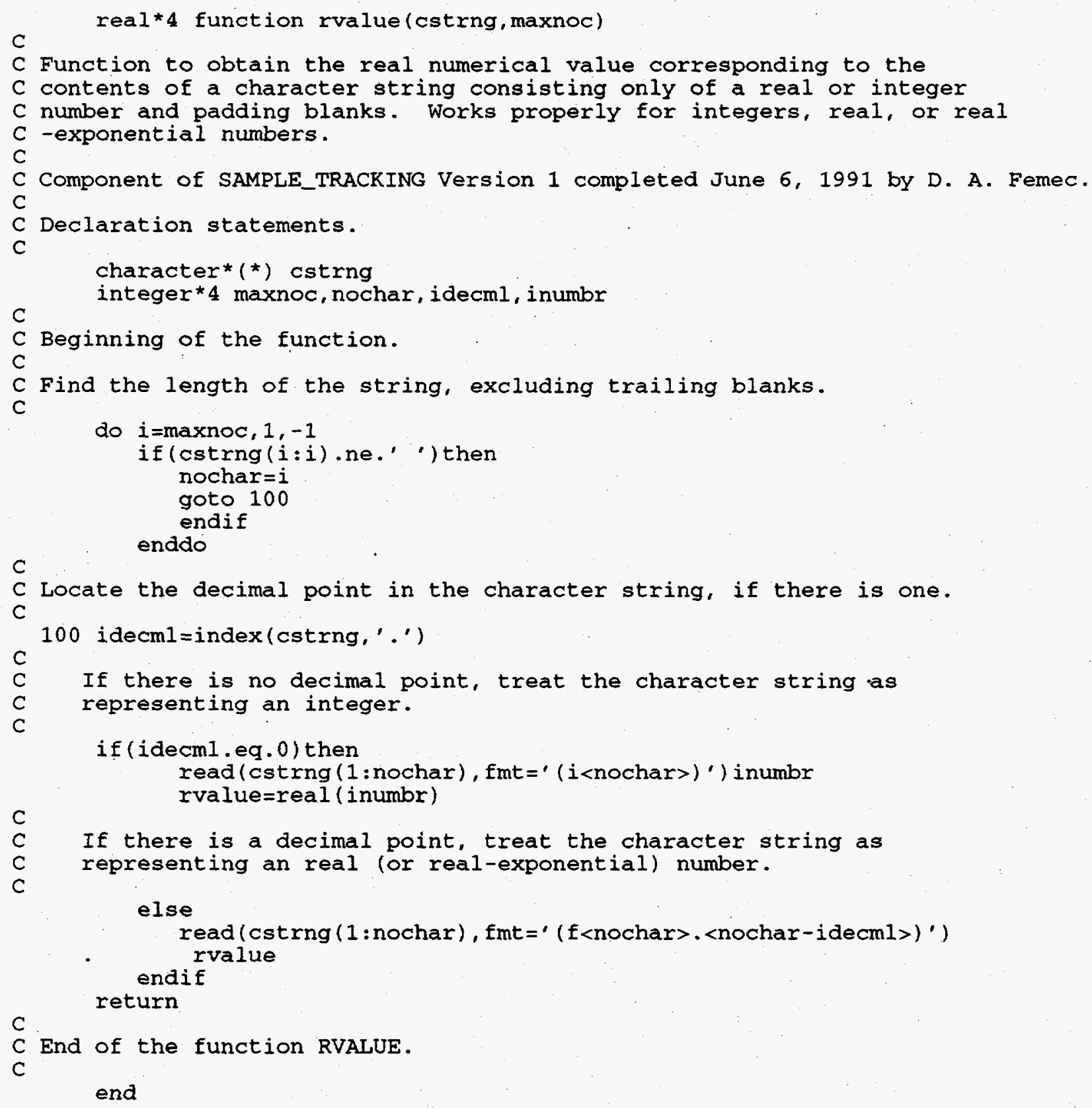




\section{N. CVALUE - FORTRAN ROUTINE FOR NUMERICAL-TO- CHARACTER STRING VALUE CONVERSIONS}

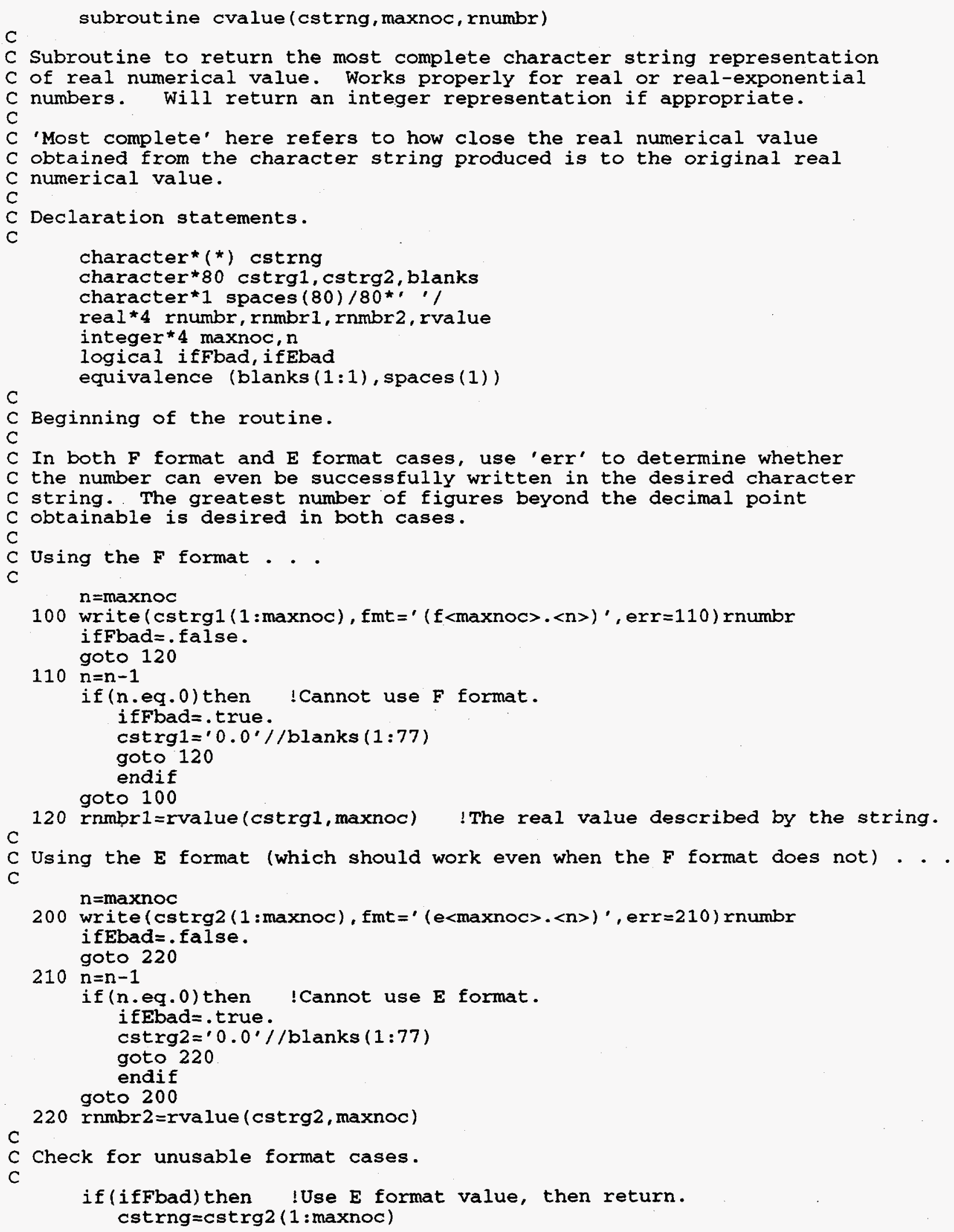

if(ifFbad)then !Use $\mathrm{E}$ format value, then return. cstrng $=$ cstrg2 $(1: \operatorname{maxnoc})$ 


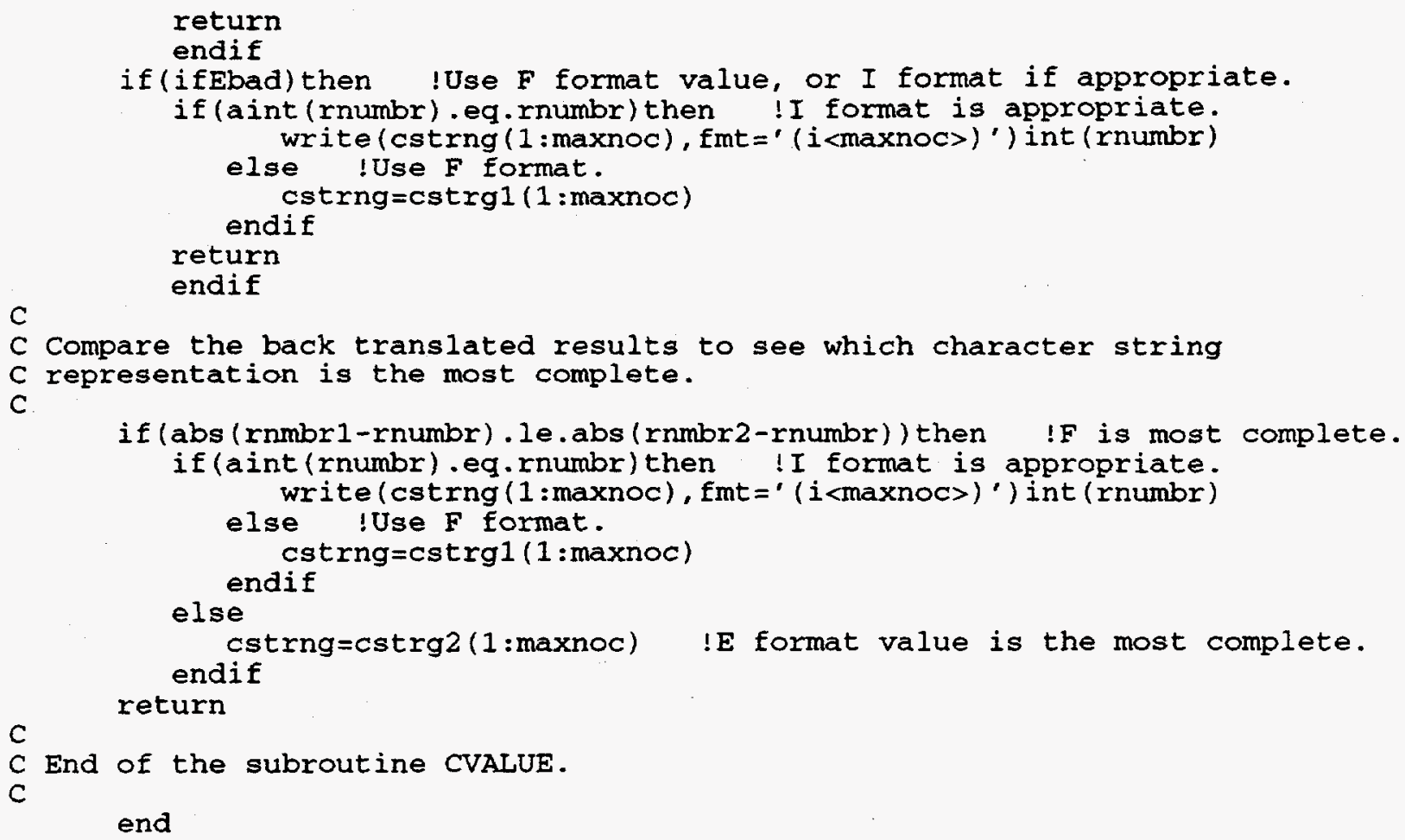




\section{O. DAYCNT AND CHRDAT - FORTRAN ROUTINES FOR CONVERSIONS BETWEEN MMDDYY DATES AND INTEGER DATE COUNTS}

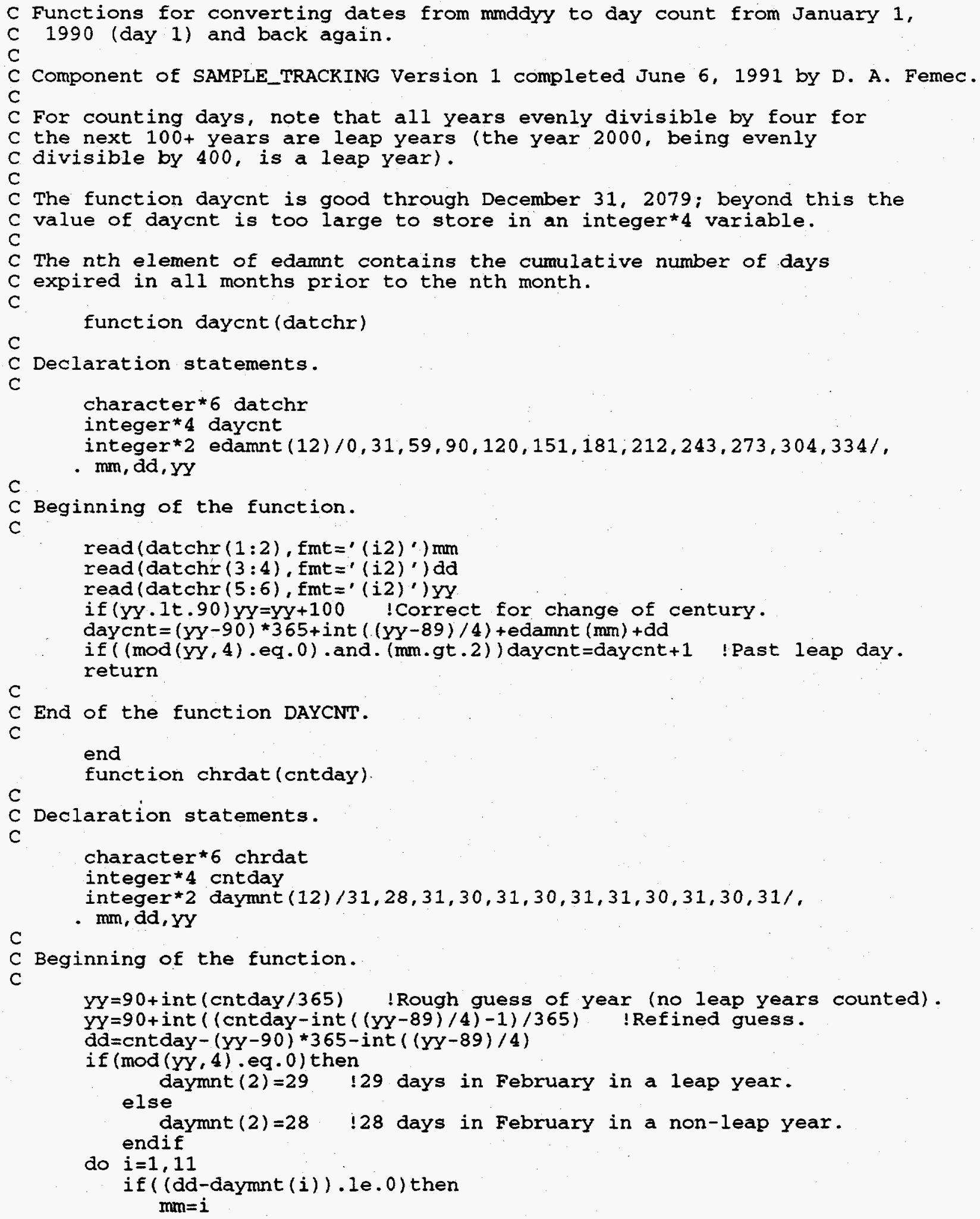




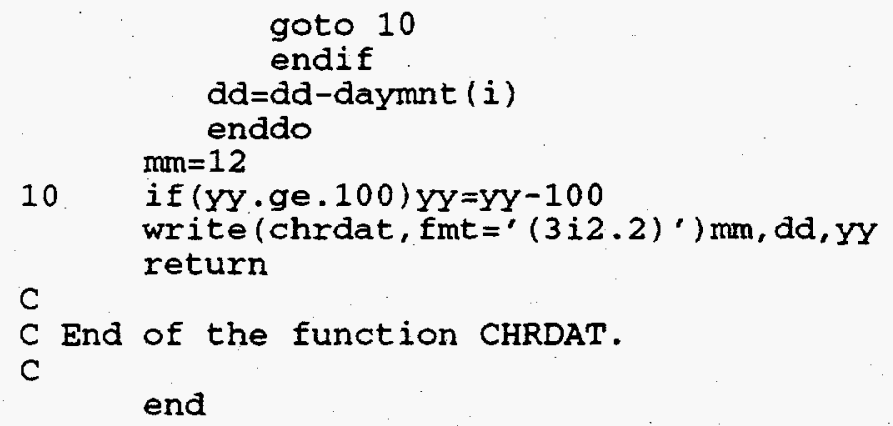




\title{
P. SRCBLD - FORTRAN ROUTINE FOR CONSTRUCTING SQL SEARCHES
}

\author{
subrout ine srcbld(comnd1, lcmnd1, comnd2, 1 cmnd2, numfld, fdat in, \\ C \\ C subroutine for building the search command. \\ C \\ C Component of SAMPLE_TRACKING Version 1 completed June 6, 1991 by D. A. Femec.




$$
\begin{aligned}
& \text { endif } \\
& \text { endif } \\
& \text { enddo } \\
& \text { C } \\
& \text { return }
\end{aligned}
$$




\section{Q. APSTRG - FORTRAN ROUTINE FOR APPENDING CHARACTER STRINGS IN A SPACE-CONSERVING FASHION}

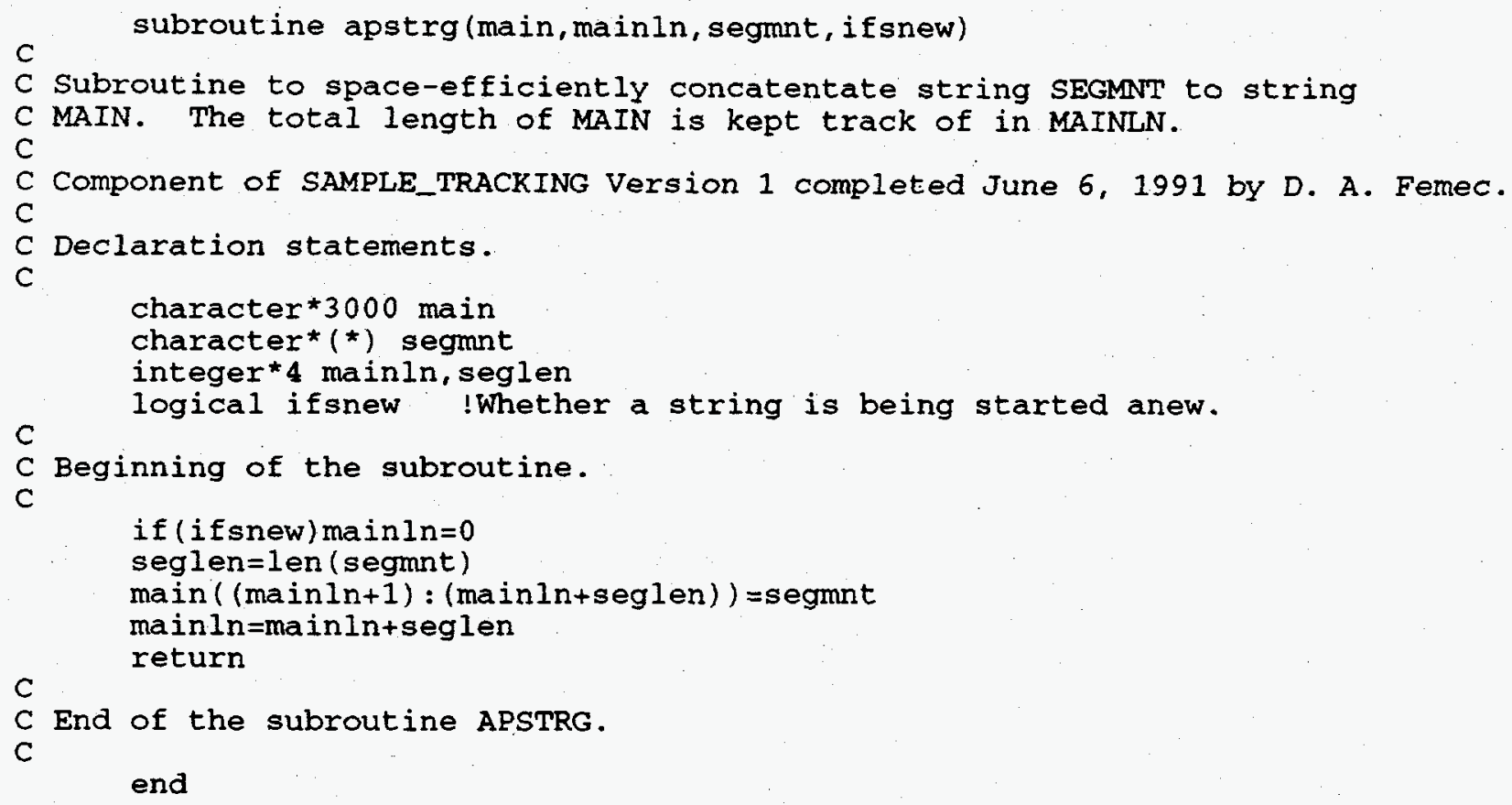

